\title{
ALL-DIGITAL CLOCK AND DATA RECOVERY ARCHITECTURES
}

\author{
by \\ Syed IRfan AHMED, M. A. SC. (EleCtricAl) \\ CARLETON UNIVERSITY, OTTAWA, ONTARIO \\ A thesis submitted to the Graduate Studies \\ in partial fulfillment of the requirements for the degree of
}

Doctor of Philosophy

Ottawa-Carleton Institute for Electrical Engineering

Department of Electronics

Carleton University

Ottawa, Ontario

September 2010 
Library and Archives

Canada

Published Heritage

Branch

395 Wellington Street Ottawa ON K1A ON4 Canada
Bibliotheque et

Archives Canada

Direction du

Patrimoine de l'édition

395, rue Wellington

Ottawa ON K1A ON4

Canada
Your file Votre référence

ISBN: 978-0-494-70537-7

Our file Notre référence

ISBN: $978-0-494-70537-7$

NOTICE:

The author has granted a nonexclusive license allowing Library and Archives Canada to reproduce, publish, archive, preserve, conserve, communicate to the public by telecommunication or on the Internet, loan, distribute and sell theses worldwide, for commercial or noncommercial purposes, in microform, paper, electronic and/or any other formats.

The author retains copyright ownership and moral rights in this thesis. Neither the thesis nor substantial extracts from it may be printed or otherwise reproduced without the author's permission.

AVIS:

L'auteur a accordé une licence non exclusive permettant à la Bibliothèque et Archives Canada de reproduire, publier, archiver, sauvegarder, conserver, transmettre au public par télécommunication ou par l'Internet, prêter, distribuer et vendre des thèses partout dans le monde, à des fins commerciales ou autres, sur support microforme, papier, électronique et/ou autres formats.

L'auteur conserve la propriété du droit d'auteur et des droits moraux qui protège cette thèse. $\mathrm{Ni}$ la thèse ni des extraits substantiels de celle-ci ne doivent être imprimés ou autrement reproduits sans son autorisation.
In compliance with the Canadian Privacy Act some supporting forms may have been removed from this thesis.

While these forms may be included in the document page count, their removal does not represent any loss of content from the thesis.
Conformément à la loi canadienne sur la protection de la vie privée, quelques formulaires secondaires ont été enlevés de cette thèse.

Bien que ces formulaires aient inclus dans la pagination, il n'y aura aucun contenu manquant.

\section{Canada}




\section{Abstract}

Clock and Data Recovery (CDR) circuits form an indispensable part of a Serializer-Deserializer system. Once the equalizer removes the distortion, the CDR circuit can recover the incoming data. There are two main types of CDR circuits-digital and analog. This classification is based on the phase detection mechanism employed. Several challenges emerge in this regard. A linear phase detector mechanism is not realizable at high speeds due to technological constraints. A digital phase detector results in a system that is dependent on the jitter distribution of the input signal. A compromise between the two extremes is to employ oversampling in the phase detector.

Exact equations have been derived in this dissertation for an oversampling phase detector with a general oversampling ratio and inter-phase spacing in the presence of random, deterministic and asymmetric jitter. An interactive Mathematica ${ }^{\circledR}$ program using symbolic analysis is developed for this purpose. These equations can be used in a behavioral simulator to perform system-level analysis at an early design stage using phase domain simulation methods.

A novel Multiple-Rotating-Clock-Phase (MRCP) oversampling all-digital data recovery architecture has been proposed and implemented on a field-programmable device. This architecture uses a multi-phase oscillator. A stable and robust data detection window is derived from the oscillator phases that also rotate to track the data.

It is not efficient or possible to simulate a CDR system at gigabit per second data

rates in a transient fashion. In this work, novel simulation techniques have been presented to reduce the transient simulation time for jitter tolerance characterization. The main idea is that the maximum slope of the jitter sinusoid is the reason for transient bit errors and the entire sinusoid need not be used. 


\section{Acknowledgments}

I wish to thank my thesis supervisor, Professor Tadeusz Kwasniewski, for his guidance, support and patience. His insight into the subject and his ability to simplify difficult concepts and put them into perspective has been instrumental in the completion of this work. I wish him all the best.

I would like to extend my gratitude to the members of the defence committee: Dr. Valek Szwarc (CRC, Ottawa), Dr. Voicu Groza (SITE, University of Ottawa), Dr. Jurek Sasiadek (Dept. of Mech. \& Aero. Engineering), Dr. Ralph Mason \& Dr. Calvin Plett (Dept. of Electronics). They have invested considerable time and energy in turning this document into a dissertation. I have benefited from their vast experience over the years.

I would like to thank all the professors and staff members at the department of electronics who have helped me reach here. I owe you all. The financial support from Carleton University, TRIO, CITO, Micronet, Altera Canada, and IC fabrication support through CMC is gratefully acknowledged.

Thanks to my family and friends. They are a mere thought away. I am happy to be alive in these times for many reasons.

Syed Irfan Ahmed

Ottawa,

September, 2010 


\section{Table of Contents}

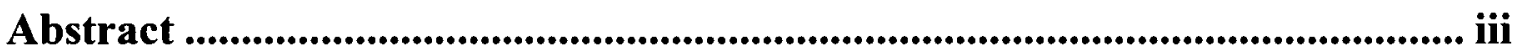

Acknowledgements .........................................................................................................

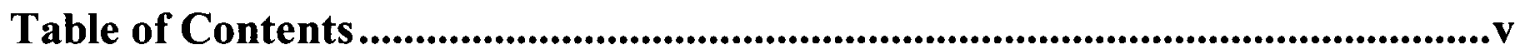

List of Symbols and Abbreviations ................................................................ xii

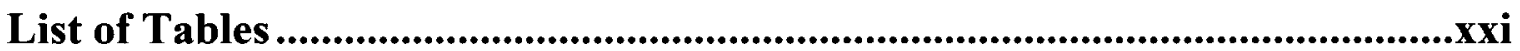

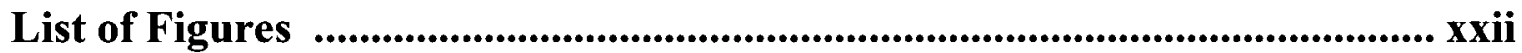

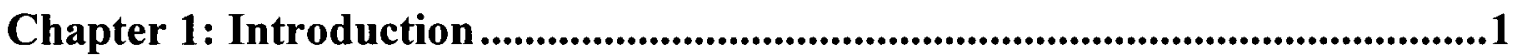

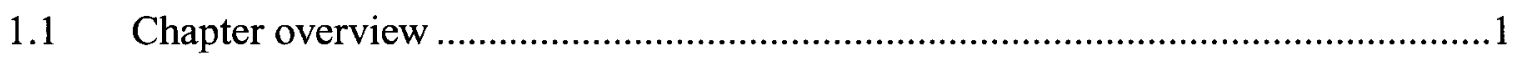

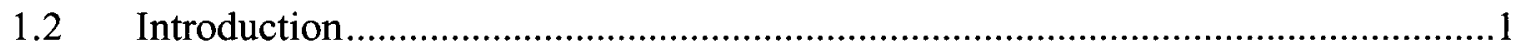

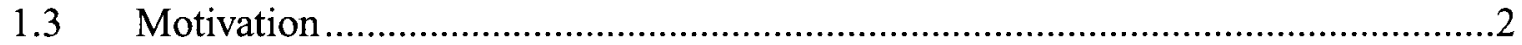

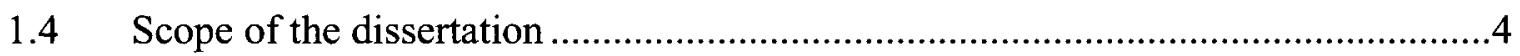

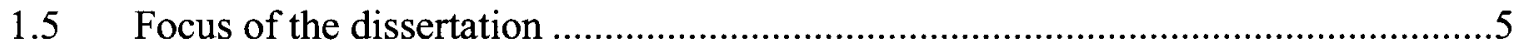

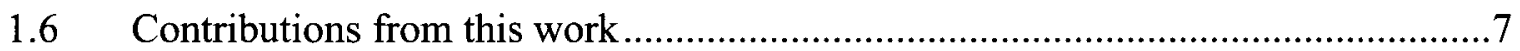

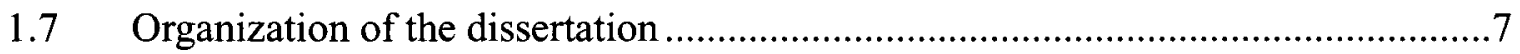

Chapter 2: SerDes Components and Interconnections .....................................9

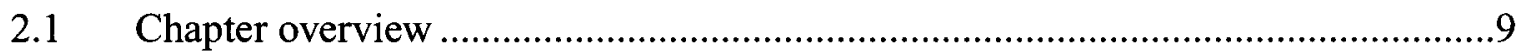

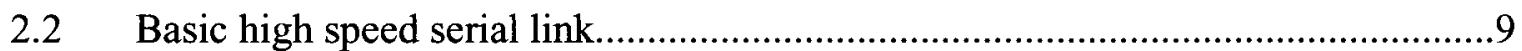

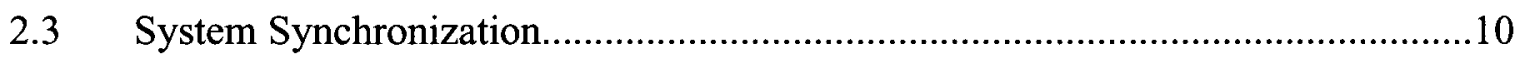

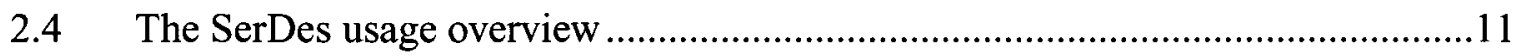

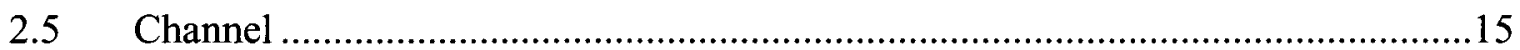




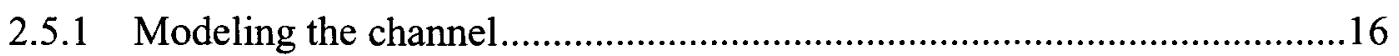

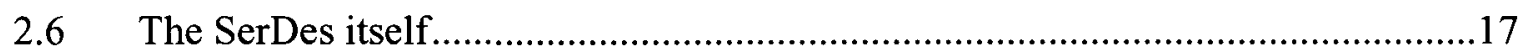

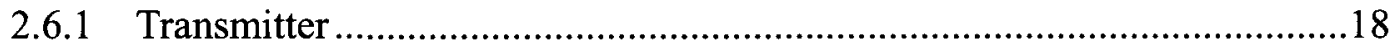

2.6.2 Data phase aligner........................................................................19

2.6.3 Decision Feedback Equalizers .............................................................20

2.6.4 Current output Digital-to-Analog Converter (iDAC) ................................22

2.6.5 Pseudorandom bit sequence (PRBS) generators .....................................23

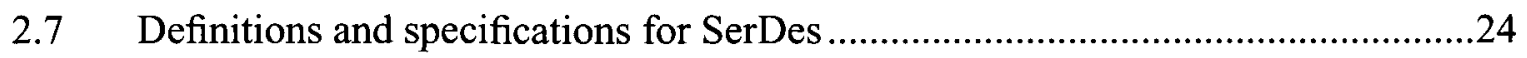

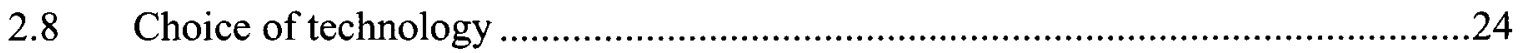

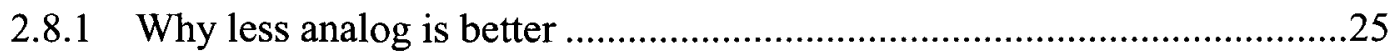

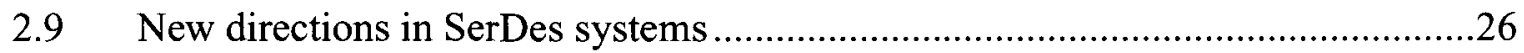

2.9.1 Wireless inter-chip SerDes architectures ..................................................26

2.9.2 Multi-tone signaling in SerDes ...........................................................27

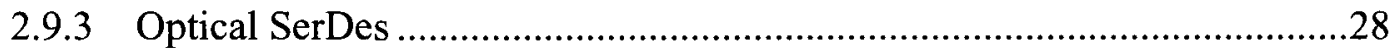

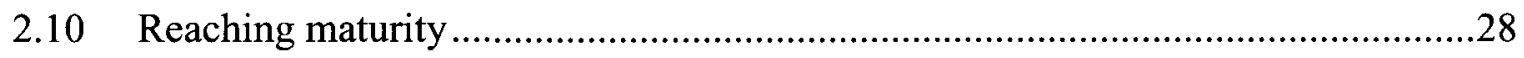

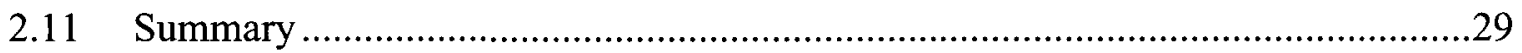

Chapter 3: CDR Architectures and Circuits.........................................................30

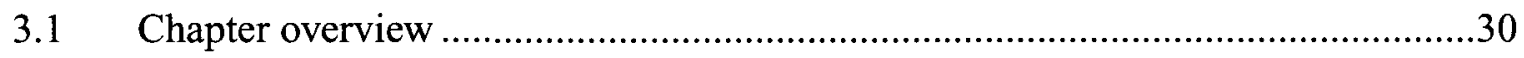

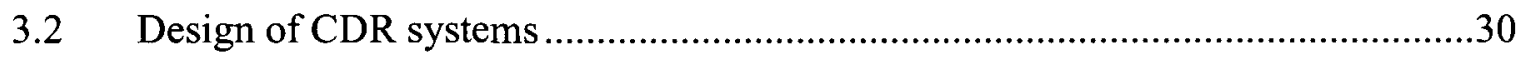

3.3 Discrete CDR circuit implementation..................................................................

3.4 Integrated CDR circuit implementations …………..........................................

3.5 Conventional PLL-based CDR systems............................................................

3.6 CDR Components as a Method of Classification ……..........................................34

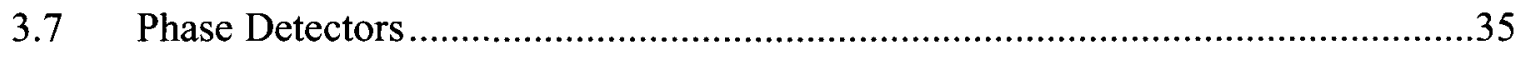

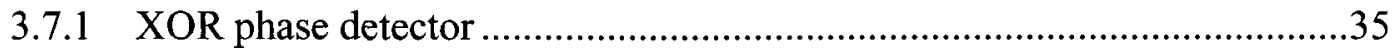


3.7.2 Tri-State phase frequency detector .........................................................36

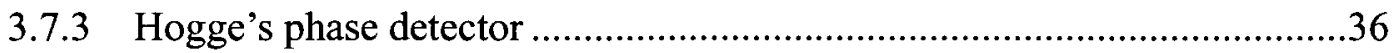

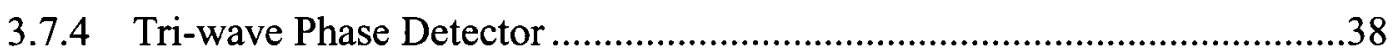

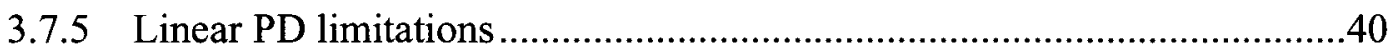

3.7.6 Half-rate linear PD ........................................................................

3.7.7 Reduced-rate linear phase detectors.......................................................

3.8 Transitioning from linear to digital phase detectors ..........................................42

3.8.1 Alexander PD or Bang-Bang PD or Early-Late PD.................................44

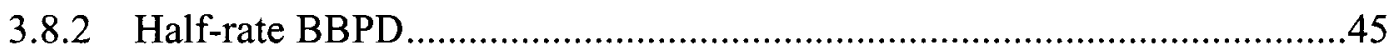

3.9 Multi-phase and multi-bit phase detectors ........................................................46

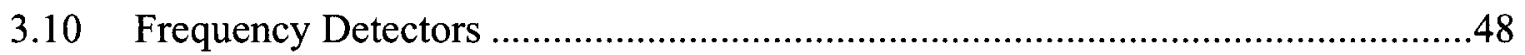

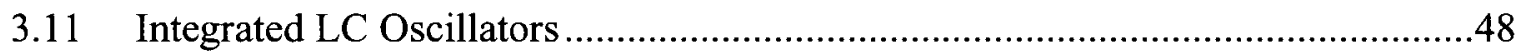

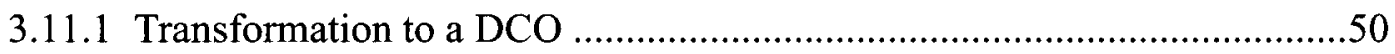

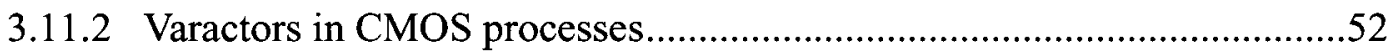

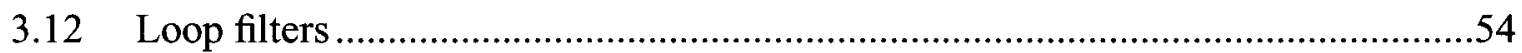

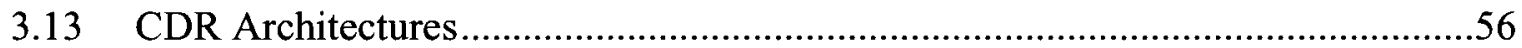

3.13.1 Reference-less, full-rate CDR architectures ……...................................57

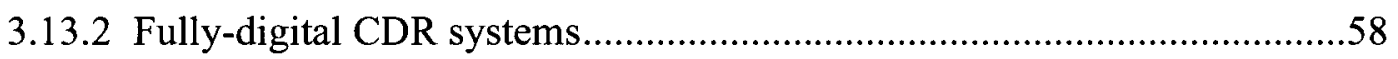

3.13.3 Full-rate CDR architectures with a local reference.....................................60

3.13.4 Full-rate binary CDR architectures ......................................................61

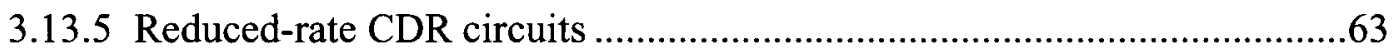

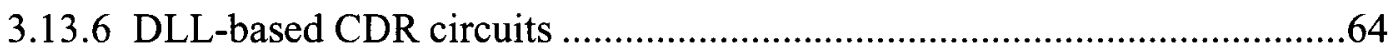

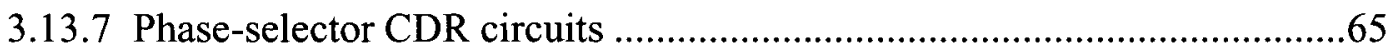

3.13.8 Injection-locked CDR architectures.........................................................66

3.13.9 Burst-Mode gated-oscillator CDR architectures......................................67

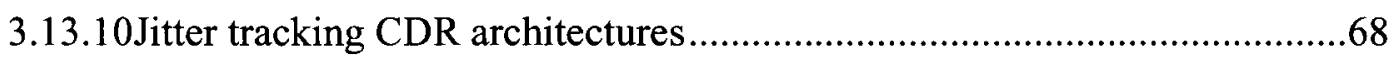

3.13.11 Variable-interval 3x-oversampling CDR architecture ...............................69 
3.13.12All-digital eye-tracking DR architecture ...............................................

3.13.13Blind Oversampling CDR ......................................................................

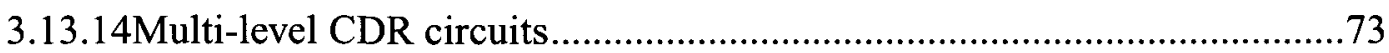

3.13.15Multi-valued DR architectures using CDMA techniques ...........................74

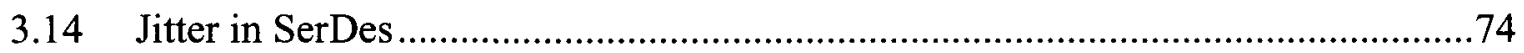

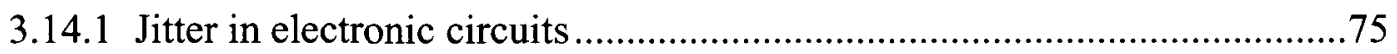

3.14.2 Jitter modeling ..................................................................................

3.14.3 Classification of jitter ............................................................................

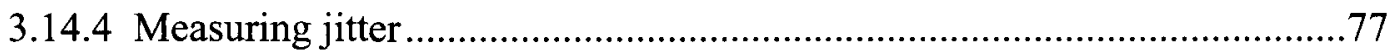

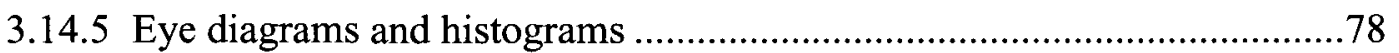

3.14.6 RMS and peak-to-peak jitter ....................................................................79

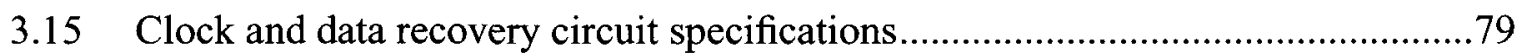

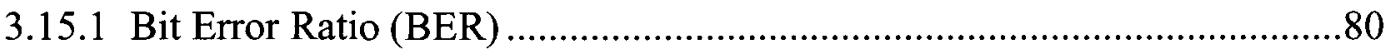

3.15.2 Bit rate, symbol rate and throughput.......................................................

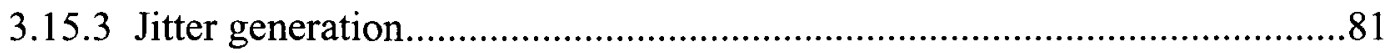

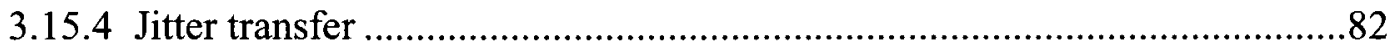

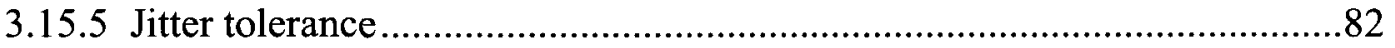

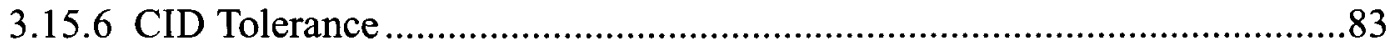

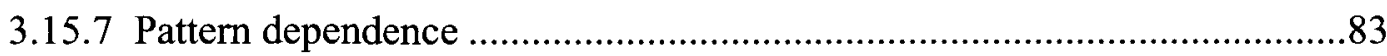

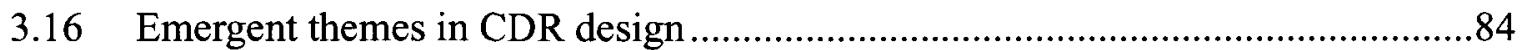

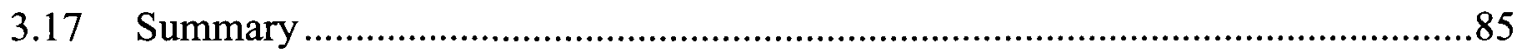

Chapter 4: Unified Phase Detector Modeling and Analysis .............................86

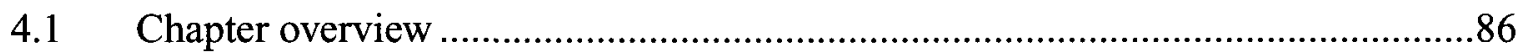

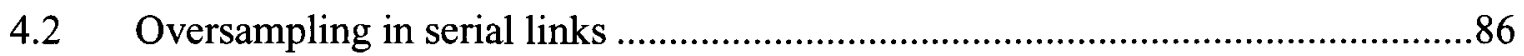

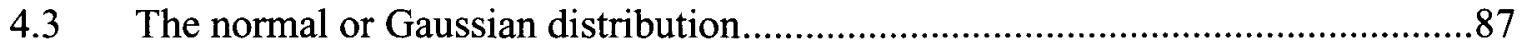

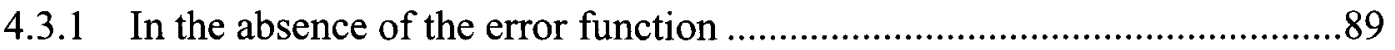


4.4 BBPD characteristics and the price of simplicity ..................................................

4.5 Transitioning from BBPD to oversampling PDs .................................................

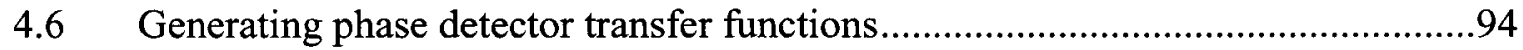

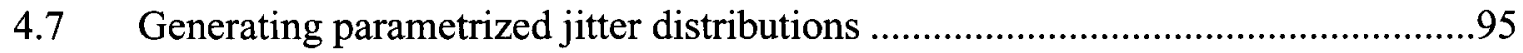

4.7.1 Asymmetric jitter distributions ............................................................95

4.8 Expected characteristics for an arbitrary NXO PD ...........................................98

$4.9 \quad$ Expected characteristics of a linear PD ........................................................100

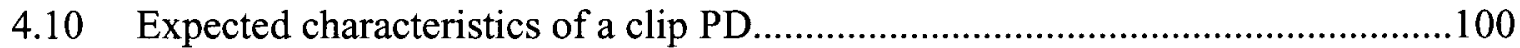

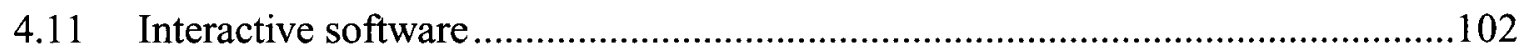

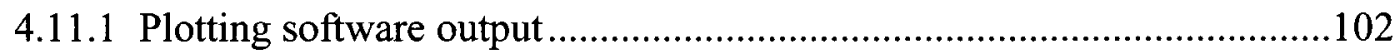

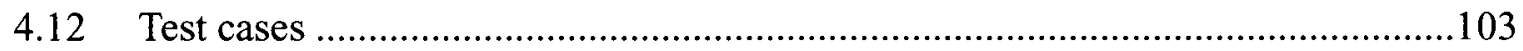

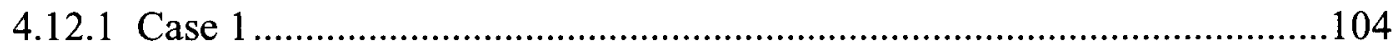

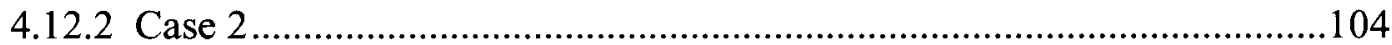

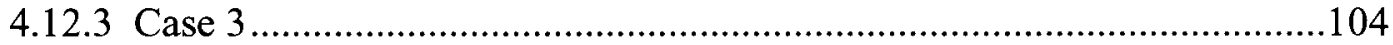

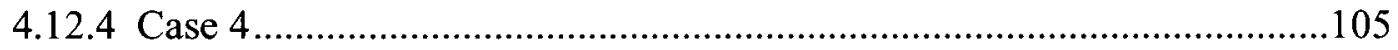

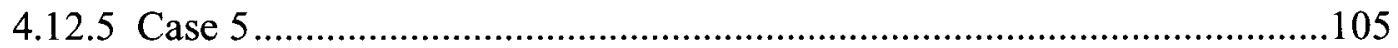

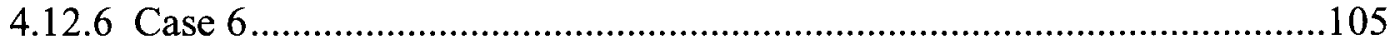

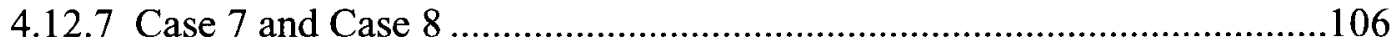

4.13 The use of random jitter as a linearizing aid.....................................................114

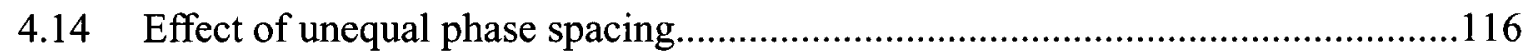

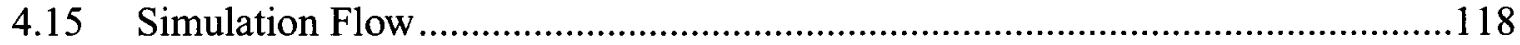

4.15.1 Transfer function analysis .....................................................................119

4.15.2 Behavioral Modeling ...............................................................................119

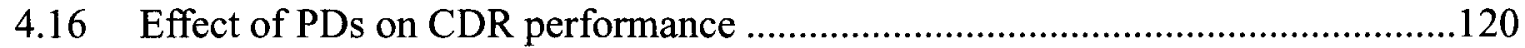

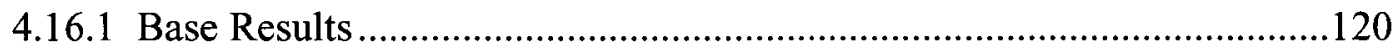

4.16.2 Effect of changing RJ and DJ separately ............................................121 
4.16.3 Robust operation with a higher OSR

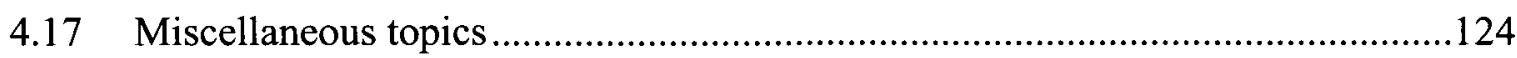

4.17.1 Using JTOL curve to debug CDR or PD anomalies .............................124

4.17.2 The case of colored noise in control systems.....................................125

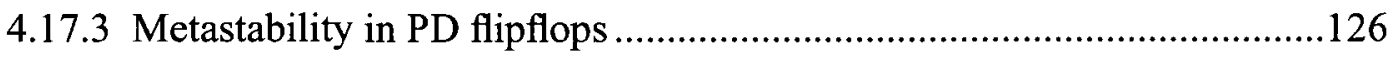

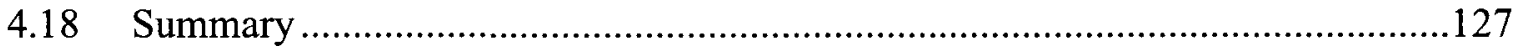

Chapter 5: An All-Digital Data Recovery Architecture..................................128

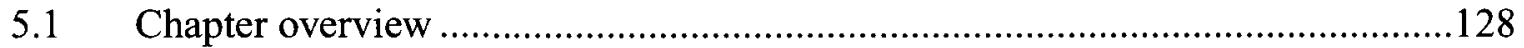

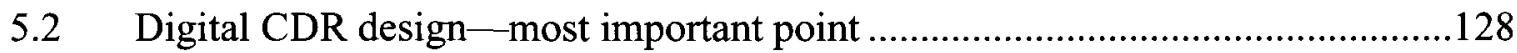

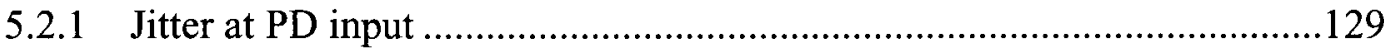

5.2.2 Phase tracking vs. frequency acquisition ........................................129

5.3 Oversampling architectures and complexity ..............................................130

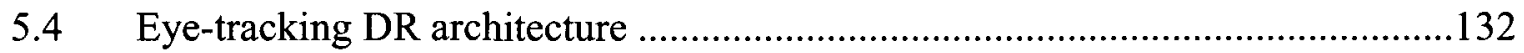

5.5 Block diagram of the MRCP-DR architecture ............................................134

5.6 Founding thoughts for the MRCP-DR Architecture .................................135

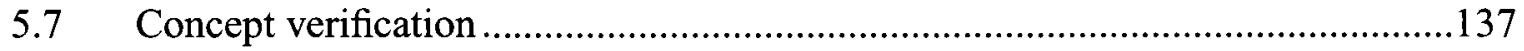

5.8 Simulating jitter tolerance ................................................................... 139

5.8.1 Phase domain jitter tolerance testbench ........................................ 140

5.8.2 Behavioral jitter tolerance testbench..............................................141

5.8.3 The basic idea of a jitter tolerance simulation ....................................143

5.8.4 Strategies to reduce JTOL transient simulation time ............................143

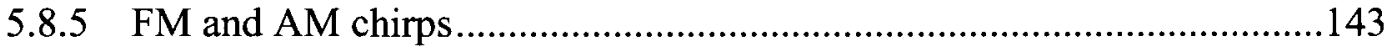

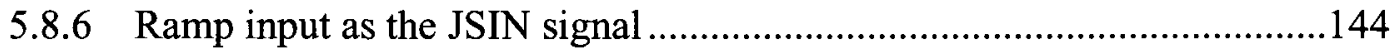

5.9 Representative simulations for the MRCP-DR ........................................145

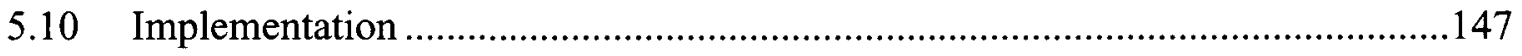




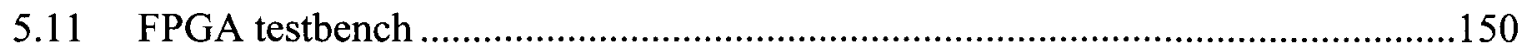

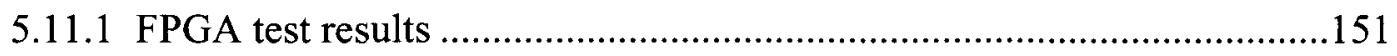

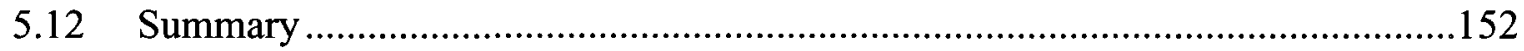

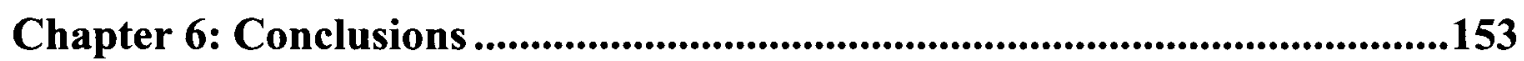

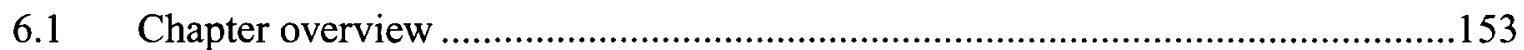

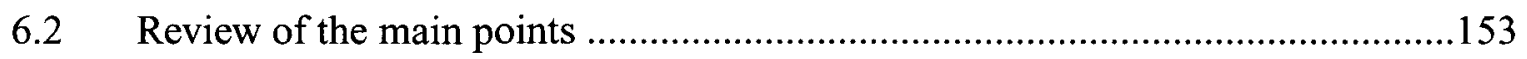

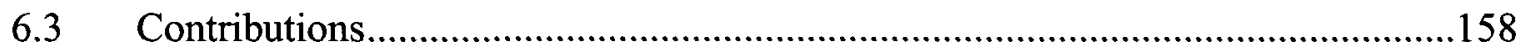

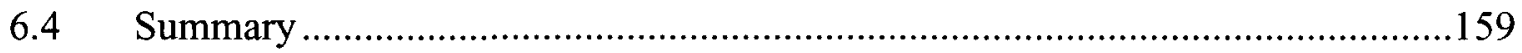

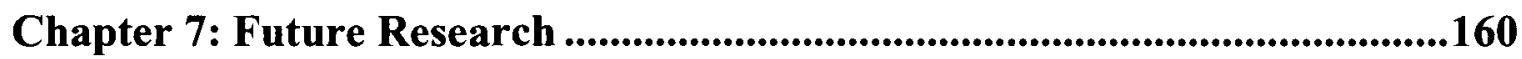

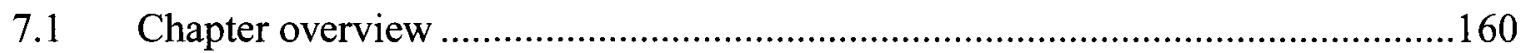

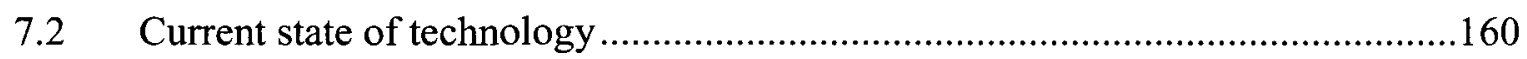

7.3 Short-term digital CDR research goals ........................................................161

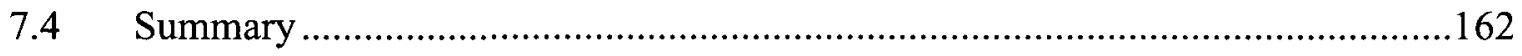

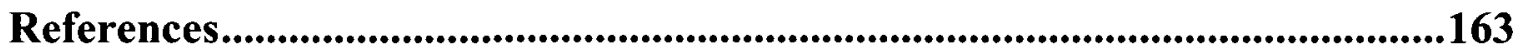




\title{
List of Symbols and Abbreviations
}

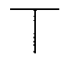 \\ $\underline{I}$ \\ $-15$ \\ $-q 5$ \\ So \\ $\sum \infty$ \\ $-p$
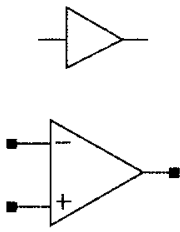 \\ Mr

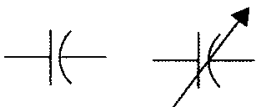 \\ $m-m$ \\ $-x-y^{\prime}$
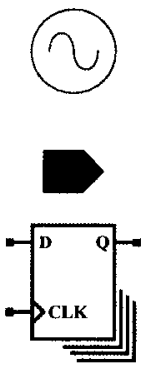 \\ $\mathbf{z}^{-1}$ \\ Power supply \\ Ground \\ NMOS \\ PMOS \\ Inverter \\ NOR gate \\ NAND gate \\ Buffer or Amplifier \\ Operational Amplifier \\ Resistor \\ Capacitor, Variable Capacitor \\ Inductor, Variable Inductor \\ Diode, Varactor \\ Oscillator \\ Port/pin (input or output) \\ D-type latch or D-type flipflop (multiple) \\ Delay element in z-domain
}




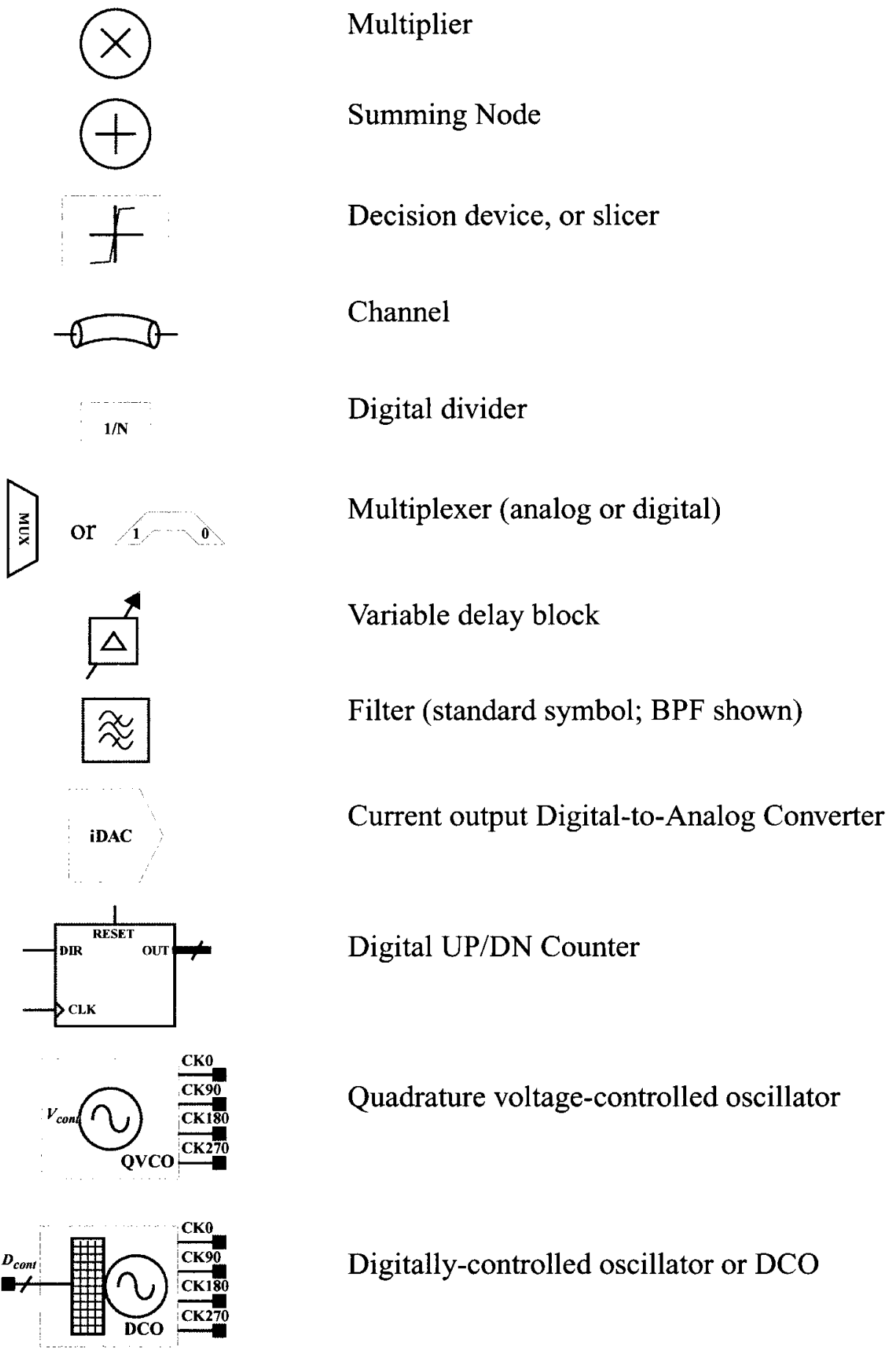

Multiplier

Summing Node

Decision device, or slicer

Channel

Digital divider

Multiplexer (analog or digital)

Variable delay block

Filter (standard symbol; BPF shown)

Current output Digital-to-Analog Converter

Digital UP/DN Counter

Quadrature voltage-controlled oscillator

Digitally-controlled oscillator or DCO 


\section{Nomenclature}

$T_{d e l} \ldots \ldots \ldots \ldots$ Bit detection interval in the Bang-Bang Phase Detector

$M \ldots \ldots \ldots$. . Phase update period

$N \ldots \ldots \ldots \ldots$ Number of clock phases

OSR ........... Oversampling ratio

$\delta \ldots \ldots \ldots \ldots \ldots$ Inter-phase spacing between PD phases

$n \ldots \ldots \ldots \ldots$ A scalar used for indexation and counting

$i \ldots \ldots \ldots \ldots$. A scalar used for indexation and counting

$k \ldots \ldots \ldots . . .$. Half of deterministic jitter

$f(\phi) \ldots \ldots \ldots \ldots$ Jitter distribution in the phase error domain

$f_{\text {asy }}(\phi) \ldots \ldots \ldots \ldots$ Asymmetric jitter distribution in the phase error domain

asy ............ Variable denoting jitter asymmetry, $0<$ asy $<1$

asy $=0.5 \ldots \ldots \ldots$ No asymmetry present in the jitter

$V_{\text {cont }} \ldots \ldots \ldots$ Control voltage input of a voltage-controlled oscillator

$V d d \ldots \ldots \ldots$. Supply voltage

$V_{t h} \ldots \ldots \ldots \ldots$ Threshold voltage

$\operatorname{erf}(x) \ldots \ldots \ldots$ Error function

$\operatorname{erfc}(x) \ldots \ldots \ldots$ Complementary error function

$Q(x) \ldots \ldots \ldots \ldots$ Q-function of the standard normal distribution

$N(\mu, \sigma) \ldots \ldots \ldots$ Gaussian distribution with mean $\mu$ and deviation $\sigma$

$N(0,1) \ldots \ldots \ldots \ldots$ Standard Normal Distribution

$\operatorname{sgn}(x) \ldots \ldots \ldots$ Signum function

* . . . . . . . Convolution operator

$\phi \ldots \ldots \ldots \ldots$. Phase error at PD input

$K_{p d} \ldots \ldots \ldots \ldots \ldots$ Phase detector gain at $\phi=0$, i.e., in locked mode

$K_{v c o} \ldots \ldots \ldots \ldots$ VCO gain at centre of VCO tuning range

$f_{\tau} \ldots \ldots \ldots \ldots$. Frequency corresponding to channel transit time

$\Theta(x) \ldots \ldots \ldots$ Unit step function

$\Theta(\phi) \ldots \ldots \ldots \ldots$. . . . . . nit step function in the phase error domain

$\Upsilon(O S R) \ldots \ldots \ldots \ldots$. . characteristics with an oversampling ratio $O S R$ 
$\tilde{\chi}(i, \phi) \ldots \ldots \ldots \ldots$. The raw NXO PD characteristics after convolution $\phi_{c} \ldots \ldots \ldots \ldots . . .6$. The shift added to get true NXO PD characteristics $\chi(i, \phi) \ldots \ldots \ldots \ldots$ True expected NXO PD characteristics with $\phi_{c}$ added $\Psi(i, \phi) \ldots \ldots \ldots \ldots$ NXO PD gain

\section{Abbreviations}

III-V . . . . . . . . Element groups for specialized semiconductors

2D, 3D........... Two (three) dimension(al)

$2 \mathrm{XO}, 3 \mathrm{XO}, \mathrm{NXO} \ldots \ldots 2 x, 3 x$ or $n-x$ oversampling

ADC .............Analog-to-Digital Converter

AGC .............Automatic Gain Control

AHDL ............. Analog Hardware Description Language

AMS $\ldots \ldots \ldots \ldots$ Analog, Mixed Signal

Al................Aluminum

AM $\ldots \ldots \ldots$........ Amplitude Modulation

ATA ..............Advanced Technology Attachment

ATE ..............Automatic Test Equipment

AWGN .............Additive White Gaussian Noise

BBPD ..............Bang-Bang Phase Detector

BER............. Bit Error Ratio

BERT. ............. Bit Error Ratio Tester (popularly called 'rate' tester)

BGA $\ldots \ldots \ldots$......... Ball Grid Array

BiCMOS ........... Bipolar Complementary-Metal-Oxide-Semiconductor

BOS.............. Blind Oversampling

BPF ..............Bandpass Filter

BPSK $\ldots \ldots \ldots \ldots$. Binary Phase-Shift Keying

BUJ ................ Bounded Uncorrelated Jitter

CDMA............. Code Division Multiple Access

CDR .............. Clock and Data Recovery

CG................ Clock Generator 
CID .............. Consecutive Identical Digit(s)

CKD_CENTER......... Centre clock phase (used in an earlier publication)

CKD_LATE ........... Late clock phase (used in an earlier publication)

CKD_EARLY ......... Early clock phase (used in an earlier publication)

Clip PD ............ A PD that clips or saturates at \pm 1 for $\phi= \pm \pi$

CLK.............. Clock (used in digital systems)

CLKC ............. Clock phase for sampling (at center)

CLKE .............. Early clock phase

CLKL ............. Late clock phase

CLKQ ............. Clock phase at $90^{\circ}$ or a quadrature clock

CMOS .............. Complementary-Metal-Oxide-Semiconductor

CML ............. Current-mode Logic

CMP ............. Chip Multi-Processor

CNT. .............. Carbon Nanotube

CP .............. Charge Pump

CPP ............. Clock phase pointer

CPU............. Central Processing Unit

$\mathrm{Cu} \ldots \ldots \ldots . . . . .$. Copper

CX................. Channel

D . . . . . . . . . Input of a D-type flipflop (or a latch)

DAC $\ldots \ldots \ldots \ldots$. Digital-to-Analog Converter

DCD .............. Duty Cycle Distortion

DCO ............. Digitally Controlled Oscillator

DDFS ............ Direct Digital Frequency Synthesizer

DEMUX ........... Demultiplexer

DFE ............ Decision Feedback Equalizer

DIMM . . . . . . . . . . Dual In-line Memory Module

DIN ............. Data In

DMA .............. Direct Memory Access

DWDM ............ Dense Wave Division Multiplexing

DDJ ............. Data Dependent Jitter 
DJ .............. Deterministic Jitter

DLL............... Delay-Locked Loop

DOUT ............. Data Out

DR.............. Data Recovery

DSP .............. Digital Signal Processing

DUT $\ldots . \ldots \ldots \ldots$. . . Device Under Test

EDA...$\ldots \ldots$. Electronic Design Automation

EMI .............. Electromagnetic Interference

ESD .............. Electro-static discharge

ET $\ldots \ldots \ldots \ldots \ldots$ Eye tracking

FBDIMM ........... Fully-Buffered DIMM

FD . . . . . . . . . Frequency detector (detection)

FEQ ............. Forward Equalizer

FEXT ..............Far-end Crosstalk

FIFO ............. First-in, First-out

FLOP.............Floating Point Operations per Second

FPGA $\ldots . . . . . .$. Field Programmable Gate Array

FSM $\ldots \ldots \ldots \ldots$. Finite State Machine

FR-4 . . . . . . . . . Fire Resistant (Fire Retardant) -4 (PCB material)

FS $\ldots \ldots \ldots \ldots \ldots$. Frequency Synthesizer

FTL . ............ Frequency tracking loop

Gbps ............. Gigabits per second

GO.............. Gated oscillator

GPS ............. Global Positioning System

HDL .............. Hardware Description Language

HF ................ High frequency

HPD. . . . . . . . . . Hogge's PD (a linear phase detector architecture)

HSSL. .............High-Speed Serial Link

IBM .............. International Business Machines (a corporation)

IC. ............ Integrated circuit

iDAC ............ Current Output DAC 
IL............. Injection locking (locked)

IP............... Intellectual Property

ISI . . . . . . . . Inter-Symbol Interference

ITRS ............. International Technology Roadmap for Semiconductors

JCLK............. Jittered Clock

JDATA . . . . . . . . . . Jittered Data

JGEN. . . . . . . . . . Jitter Generation

JSIN. . . . . . . . . . . Jitter sinusoid

JTRAN ............Jitter Transfer

JTOL ..............Jitter Tolerance

LD ................ Lock Detect

LF ................oop-filter, Low Frequency

LFSR.............. Linear Feedback Shift Register

LMS .............. Least Mean Squares (an equalization algorithm)

LNA $\ldots \ldots \ldots$......... Low Noise Amplifier

LPF ............... Low-Pass Filter

Mbps..............Megabits per second

MEMS.............Micro-Electro-Mechanical Systems

MIM ............... Metal-Insulator-Metal (a type of integrated capacitor)

MIMO .............Multiple-Input, Multiple Output

MOSFET .............Metal-Oxide-Semiconductor Field Effect Transistor

MUX..............Multiplexer

MRCP ............ Multiple-Rotating-Clock-Phase

NEXT ............ Near-End Crosstalk

NRZ. ............ Non-Return to Zero

NXO $\ldots \ldots \ldots \ldots$ A general oversampling ratio (see 3XO, 5XO etc.)

OSR ............. Oversampling ratio

PAM ............. Pulse Amplitude Modulation (as in PAM-2 and PAM-4)

PC $\ldots \ldots \ldots \ldots$......... Personal Computer

PD .............. Phase Detector

PCI .............Peripheral Component Interconnect 
PCB...$\ldots \ldots \ldots$. Printed Circuit Board

pdf . ............. probability distribution function

pdf . .............. Portable Document Format, a file format

PFD ............... Phase-Frequency Detector

PI.............. Phase interpolator (interpolating)

PJ. ..............Periodic jitter

PLL ................ Phase-Locked Loop

PLL-FS ............PLL-based Frequency Synthesizer

PN . . . . . . . . . . . Phase noise

PON...............Passive Optical Network

POSL, POSC, POSE. . . Position of the late, center, early clock phases

pp.............. peak-to-peak

PRBS.............Pseudo-Random Bit Sequence

PTL . ............. Phase tracking loop

PVT.............. Process, Voltage and Temperature

Q .............. Output of a flipflop

QVCO........... Quadrature voltage-controlled oscillator

r.v. .............. random variable

RAM ............. Random Access Memory

RJ . . . . . . . . . Random Jitter

RX.............. Receiver

R\&D ............Research and Development

S2P $\ldots \ldots \ldots \ldots \ldots$ Serial-to-Parallel

SAS .............. Serial-Attached SCSI

SAW .............. Surface Acoustic Wave

SATA............. Serial Advanced Technology Attachment

SCSI ............. Small Computer System Interface

SFI-5 ........... SerDes Framer Interface, a backplane specification

SiP.............. System-in-Package

SoC $\ldots \ldots \ldots$....... System-On-Chip

SDT .............. Serial Data Transmission 
SerDes............. Serializer-Deserializer

SRJ .............. Sub-rate jitter

TDR............. Time Domain Reflectometry

TX.............. Transmitter

UI $\ldots \ldots \ldots \ldots$. Unit Interval

USB............. Universal Serial Bus

VCDL ............ Voltage-Controlled Delay Line

VCO .............. Voltage-Controlled oscillator

VDSM............. Very Deep Submicron

VLSI ............. Very Large Scale Integration 


\section{List of Tables}

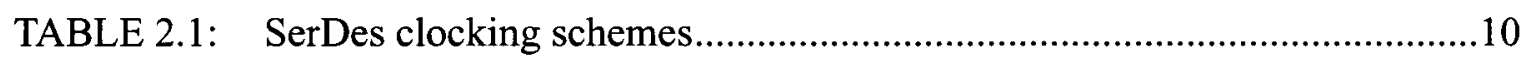

TABLE 2.2: Channel types and data rates..................................................................15

TABLE 4.1: Test Cases - PD performance evaluation ……...........................................103 


\section{List of Figures}

Figure 1.1 Hierarchy and scope of the dissertation ...................................................

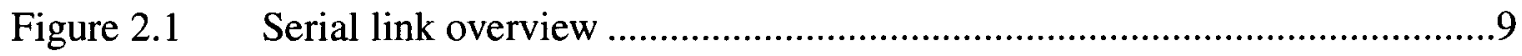

Figure 2.2 Flip chip die on a BGA package (cross section) ........................................11

Figure 2.3 Typical off-chip SerDes usage ……….....................................................12

Figure 2.4 Typical SerDes Usage in SoC modules ....................................................13

Figure 2.5 On-chip data transfer (a) Parallel vs. (b) SerDes .......................................14

Figure 2.6 A typical FR-4 backplane channel ..........................................................16

Figure $2.7 \quad$ SerDes block diagram ......................................................................18

Figure 2.8 3-tap Pre-emphasis block diagram........................................................18

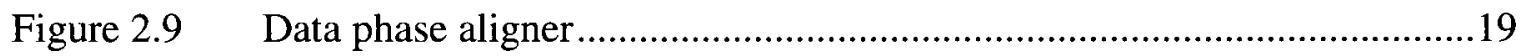

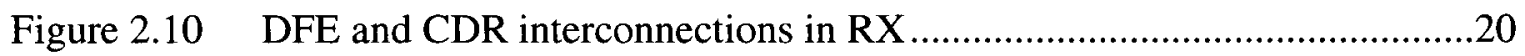

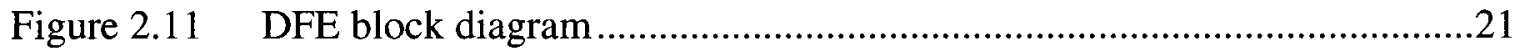

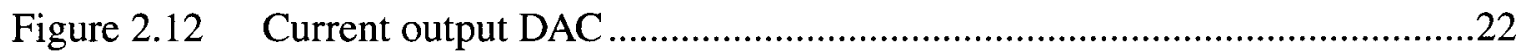

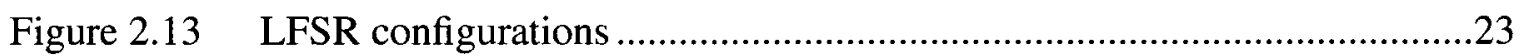

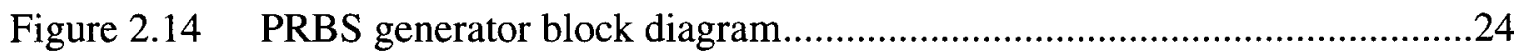

Figure 2.15 Analog design trade-offs across many dimensions ...................................25

Figure 2.16 Multi-tone SerDes block diagram ...........................................................27

Figure $3.1 \quad$ Discrete CDR implementation ...............................................................

Figure $3.2 \quad$ CDR concept diagram ............................................................................

Figure 3.3 Conventional CDR block diagram ............................................................32

Figure $3.4 \quad$ Challenges in designing CDR circuits .....................................................3

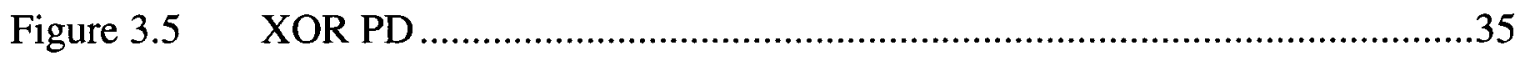

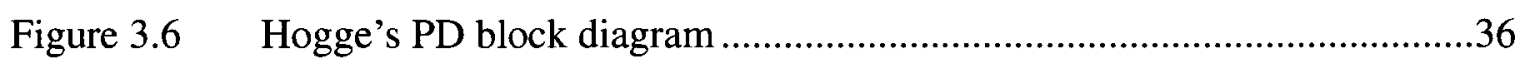

Figure $3.7 \quad$ Hogge's PD operation (open loop) ……................................................. 
Figure 3.8 Hogge's PD style linear CDR response

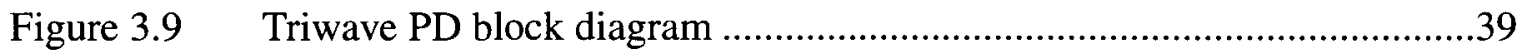

Figure 3.10 Triwave PD style linear CDR response ..................................................39

Figure 3.11 Half-rate linear PD block diagram .................................................................41

Figure 3.12 Half-rate linear PD block diagram and operation ......................................41

Figure 3.13 Alexander PD block diagram and operation ...........................................44

Figure 3.14 Half-rate Binary PD and operation …………......................................46

Figure 3.15 Multi-phase detectors and operation.......................................................46

Figure 3.16 Rotational frequency detectors ………..................................................48

Figure 3.17 From VCO to DCO - The paradigm shift …….........................................49

Figure 3.18 Noise gateways into the oscillator ………............................................50

Figure 3.19 MOS varactor characteristics .................................................................52

Figure $3.20 \quad$ Loop filter evolution........................................................................5

Figure 3.21 Linear CDR (a) single VCO control (b) dual VCO control........................57

Figure 3.22 Inclusion of data converters in CDR loops .............................................58

Figure 3.23 An all-digital DCO-based CDR architecture .........................................59

Figure 3.24 CDR architectures with a local reference …..........................................61

Figure 3.25 Full-rate, binary CDR architectures ....................................................62

Figure 3.26 Half-rate CDR architectures ..............................................................63

Figure 3.27 CDR system with a wideband DLL with narrowband PLL.......................64

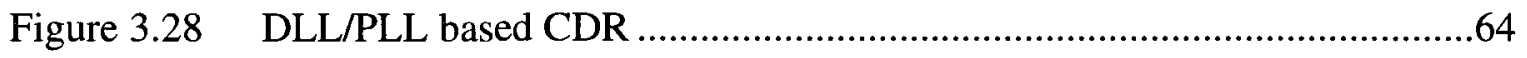

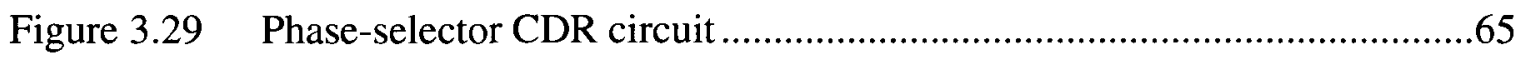

Figure $3.30 \quad$ Phase-interpolating CDR architecture ...................................................66

Figure 3.31 Injection-locked CDR Circuit ……….................................................66

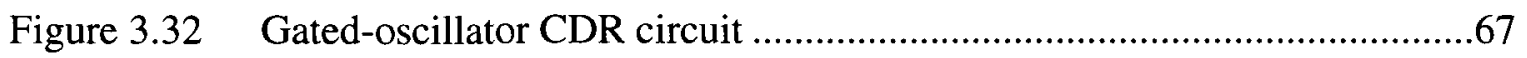

Figure 3.33 Effect of asymmetric jitter on CDR operation .......................................68 
Figure 3.34 Variable interval oversampling CDR architecture

Figure 3.35 All-digital eye-tracking DR architecture ...............................................70

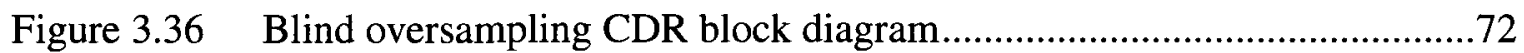

Figure 3.37 Modeling jitter components ..........................................................................76

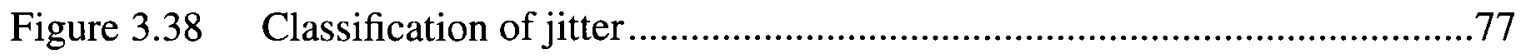

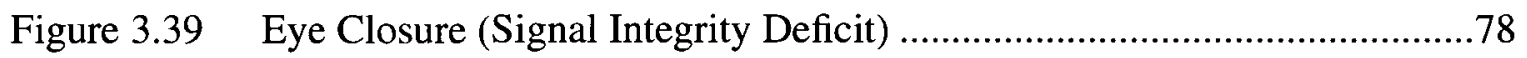

Figure 3.40 Typical jitter transfer characteristics ......................................................82

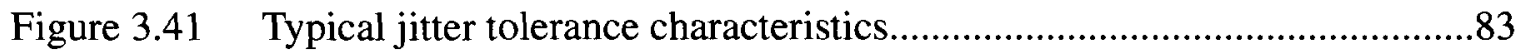

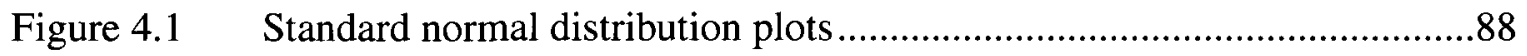

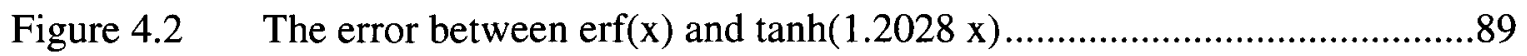

Figure $4.3 \quad$ Expected BBPD characteristics ………......................................................90

Figure 4.4 Transitioning from BBPD to linear............................................................93

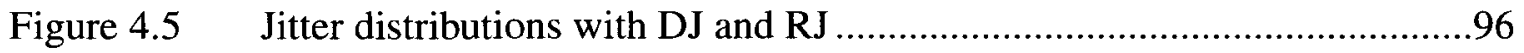

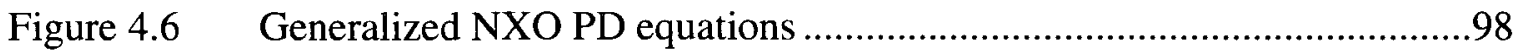

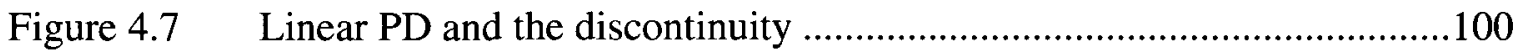

Figure 4.8 Clip PD as an analytical device …........................................................101

Figure 4.9 Case 1 - RJ only, maximal phase spacing …...........................................107

Figure 4.10 Case 2 - RJ + DJ, maximal phase spacing ............................................108

Figure 4.11 Case 3 - Enough DJ to cause histogram flattening …..............................109

Figure 4.12 Case 4 - PM modulation style DJ ……..................................................110

Figure 4.13 Case 5 - Excessive RJ + DJ ................................................................111

Figure 4.14 Case 6 - Excessive RJ + DJ with asymmetry ..........................................112

Figure 4.15 Case 7 and Case 8 - Change of inter-phase spacing …...........................113

Figure 4.16 Using RJ to linearize the PD characteristics ............................................114

Figure 4.17 Linearization in presence of DJ ........................................................115

Figure 4.18 Effect of unequal phase spacing........................................................117 
Figure 4.19 Validity of transfer function analysis

Figure 4.20 Base results for JTOL and JTRAN ……..........................................120

Figure 4.21 Effect of jitter distribution on JTOL ……….............................................121

Figure 4.22 Robustness due to a higher oversampling ratio ......................................123

Figure 5.1 Eye-tracking and and variable-interval oversampling CDR concepts .....131

Figure $5.2 \quad$ Effect of $T_{d e l}$ on JTOL …………......................................................133

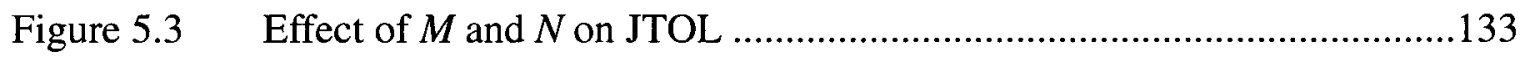

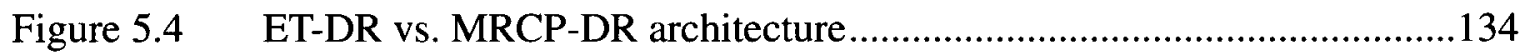

Figure 5.5 Three data phases vs. three clock phases ................................................135

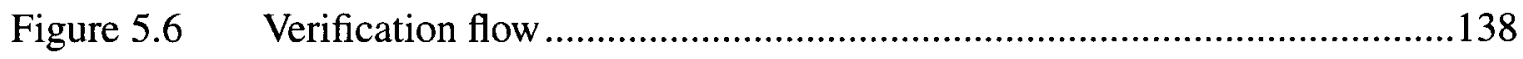

Figure 5.7 Comparing the tracking performance using Matlab ..............................139

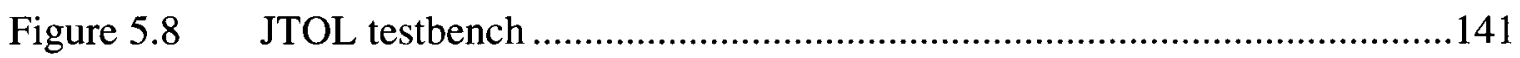

Figure 5.9 Functional verification of JTOL using Verilog-A …................................142

Figure 5.10 AM and FM chirps on the JSIN signal .................................................144

Figure 5.11 Ramp input as the JSIN signal ...........................................................145

Figure 5.12 Jitter tolerance - MRCP-DR architecture .................................................146

Figure $5.13 \quad$ Jitter tolerance - MRCP-DR (continued) …............................................147

Figure 5.14 Comparison of tracking performance ………..........................................147

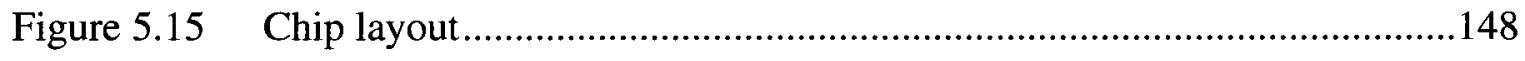

Figure 5.16 Top-level chip block diagram................................................................148

Figure 5.17 Die photograph and laser surgery close-up.............................................149

Figure 5.18 Partial measured results for the custom chip ........................................149

Figure 5.19 FPGA test setup …………….....................................................150

Figure 5.20 Tracking results and phase generation ...................................................151

Figure 5.21 Introducing RJ using random edge selection .........................................152

Figure 7.1 Proposed DCO-based CDR architecutre ..................................................161 
"There is nothing more difficult to take in hand, more perilous to conduct, or more uncertain in its success, than to take the lead in the introduction of a new order of things."

Niccolo Machiavelli

\subsection{Chapter overview}

This chapter introduces the dissertation and explains its organization. The motivation for this work is discussed and the contributions are summarized. Serializer-Deserializer (SerDes) architectures were introduced in the 1990s and even at their current level of commercial maturity in 2010 , these remain an area of active research and development (R\&D). The Clock and Data Recovery (CDR) [2] unit forms an indispensable subsystem of the SerDes macro in addition to the decision feedback equalizer (DFE) [86]. The DFE opens the vertical eye opening and the CDR circuit opens the horizontal eye opening to enable error-free detection of incoming data symbols that have been corrupted by the jitter acquired in the channel.

\subsection{Introduction}

Parallel data transmission requires more area, power, pads, pins, deskewing circuitry, generates heat and occupies a large footprint on chips and printed circuit boards (PCB). Serial data transmission is cost-effective and has, therefore, widely replaced parallel data transmission as the dominant system-level communication strategy in off-chip and on-chip scenarios. The parallel-to-serial paradigm shift was the logical outcome of the maturity of Complementary-Metal-Oxide-Semiconductor (CMOS) technology that 
enabled large scale digital systems design. The need for sharing data on-chip and off-chip is a dominant driver in the personal computer (PC) and telecommunication industries.

For high-speed serial link (HSSL) design [23] [52], significant complexity needs to be managed at the receiver side, where the corrupted data symbols are being received. The anomalies that introduce the corruption of data symbols are lumped together under the titles 'jitter' and 'attenuation and inter-symbol interference (ISI)'. The DFE ameliorates the ISI thereby improving the vertical eye opening; the automatic gain control counters attenuation while the CDR circuit rejects the jitter to improve the horizontal eye opening.

The digital Integrated Circuit (IC) design techniques [11][142] allow the re-use of intellectual property (IP) and are, therefore, used to manage complexity for large mixedsignal IC design projects. CMOS technology is credited for the digital revolution although its legendary cost-effectiveness has become an increasingly relative notion.

It is the goal of this dissertation to analyze the design and simulation trade-offs for all-digital, CMOS oversampling CDR architectures, in order to propose cost-effective and portable alternatives.

\subsection{Motivation}

Serial systems have emerged as the cost-effective way to manage the information super highways. Bandwidth is a precious resource and serial communication allows for packet-based multi-user and multi-purpose communication systems such that the available bandwidth is optimally utilized. The HSSL revolution has been more tangible for central processing unit (CPU), PC and server markets. Computer peripherals use the Universal Serial Bus (USB 2.0 and USB 3.0) or the FireWire Interface. Hard drive interfaces have moved on from the legacy parallel ATA to Serial ATA, SAS and Fibre Channel. Fully Buffered Dual In-line Memory Modules (FBDIMM) have been phased out after much hype due to thermal issues but might find their niche again in future memory architectures. 
Sizable blocks of data in graphic processing units are moved around in a serial fashion to realize highly realistic 3D display systems. Serial buses using information packets such as Infiniband ,Hypertransport and PCI-e dominate the CPU landscape. The parallel to serial transformation at the backend using fiber-optic hardware is more astounding and is exclusively serial for the medium- and long-haul markets. The unveiling by Intel corporation of Light Peak ${ }^{\mathrm{TM}}[159]$ for a scalable 10-100 Gbps interconnect technology signals a new beginning for computer peripherals. The interconnect is now moving from electrical to optical substrates to cope with computation and bandwidth requirements [113]. It can be surmised, therefore, that serial link technology is here to stay.

Serial links can be treated as a utility that can transmit packets from different sources to different destinations. Furthermore, system-level upgrades require minimal amounts of hardware and software changes. In comparison, for example, increasing the word length for a parallel system requires major software and hardware changes. For serial links, redundancy can be incorporated routinely with equal facility and data routing remains possible at lower bandwidth if a certain link shuts down for maintenance or repair. This flexibility is absent in parallel links. Serial link components in CMOS technologies are the main focus of this work. The outlooks for the semiconductor industry are available at the International Technology Roadmap for Semiconductors (ITRS) website [158].

Serial link solutions have reached fundamental limits just like any other electronic system. Despite the several advantages cited above, the industry unceremoniously abandoned the FBDIMM in less than three years after launch around 2008, since such memory modules do not alleviate the thermal footprint and other technological problems in PC and server markets. Instead dual-channel and triple-channel memory schemes have been brought back, albeit, more motherboard area is required to implement such solutions. This example illustrates that even though the serial links are more cost-efficient, the outcome might not always be in their favor when all things are considered. 


\subsection{Scope of the dissertation}

The scope of the dissertation is pictorially depicted in Figure 1.1. The reason for these choices have been explained, as appropriate, throughout the disseration.

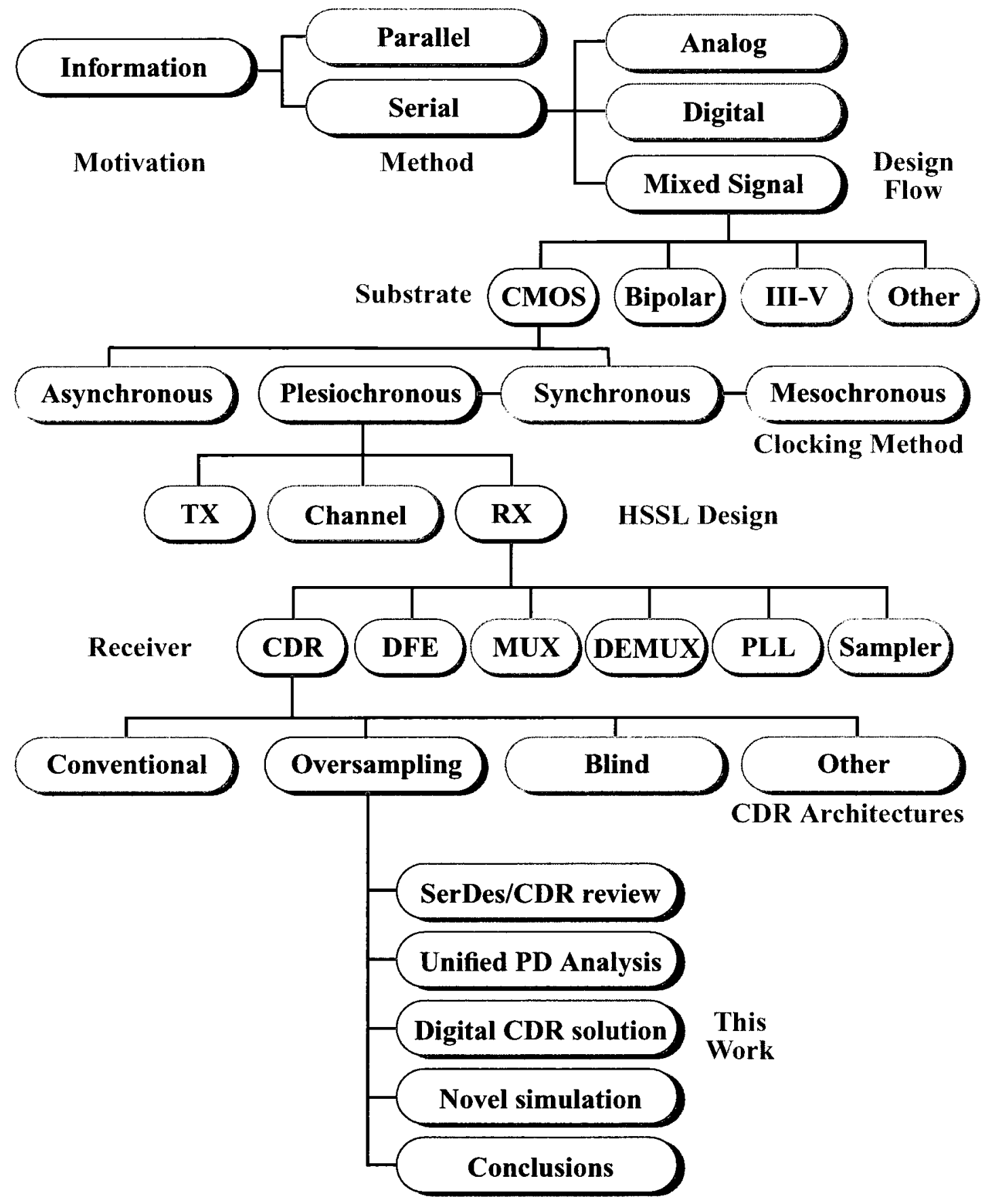

Figure 1.1 Hierarchy and scope of the dissertation 


\subsection{Focus of the dissertation}

The focus of this dissertation is the investigation and simulation of oversampling, all-digital CMOS CDR architectures. Conventional CDR architectures employ analog PLL circuits [2]. A major and closely related class of circuits utilizing analog PLLs is the frequency synthesizer (FS). The traditional knowledge of PLL-FS ports over well to the CDR circuits but not without addressing a fundamental difference between the two systems, i.e., the type and quality of their respective input signals. For a CDR circuit:

- The phase detector (PD) [25] works at the frequency of the incoming data. A PD is a power-hungry device in a CDR system.

- Even if the samplers can be designed in a given technology at full speed, the vanishing narrow pulses near lock to drive the charge pump (CP) cannot be handled with the same technology and result in a deadzone that creates jitter [32].

- The Non-Return to Zero (NRZ), Pseudo Random Binary Sequence (PRBS) acquires jitter from the channel—both deterministic jitter (DJ) and random jitter (RJ). Hence, a wide bandwidth PLL results in excessive noise and degrades the CDR circuit's timing margin and the jitter transfer (JTRAN) specification.

- There are missing transitions in the incoming data during which the PLL briefly wanders away from the optimum frequency due to charge pump leakage.

In comparison, a PLL-FS locks to a low frequency crystal reference source that is stable and has no missing transitions. It is evident that conventional knowledge of synthesizer architectures cannot simply be extended to the CDR domain, even though a PLL forms the core of both systems.

The averaged bang-bang phase detector (BBPD) characteristics change with the statistics of the input signal [33][34]. This issue is analyzed in detail in this dissertation. A trade-off can be implemented in this regard with the provided analysis such that the oversampling ratio $(O S R)$ and inter-phase spacing parameter $(\delta)$ can be used to ensure maximally stable PD characteristics in the presence of the given input jitter. 
To achieve a satisfactory Bit Error Ratio (BER), further design considerations need to be addressed.

- Analog design knowledge is a highly specialized skill that requires experience. An all-digital approach improves portability for large sections of the design. Digital IPs can often be scaled and reused in a cost-effective manner. This comment applies to the architectures as well as to individual subsystems.

- Analog PLL-based CDR designs sample the data at only one point during the unit interval (UI). Sampling the data transitions at multiple points within the UI is called oversampling and can improve CDR performance. An analysis framework for oversampling CDR systems is developed in this dissertation.

- A local multi-phase clock source locked to a clean reference can be provided at the receiver instead of locking to the jittered data signal [43][40]. This decouples the tracking performance from the locking performance, offering an additional degree of freedom. Data tracking can be implemented using multiple phases as the jitter changes from cycle to cycle.

- In order to avoid short pulses in the linear phase detector, a 2-level or an $n$-level oversampling PD (NXO PD) can be used to detect data transitions. Thus, the sampler design becomes the remaining speed bottleneck that can be alleviated by using a reduced-rate architecture, if required.

- If the jitter changes direction across the sampling point from cycle to cycle, then the sampling point need not be moved. For a system employing multiple oscillator phases, a new phase need not be selected every cycle. This reduces power consumption without degrading the BER. Analog PLL-based CDR systems provide continuous correction and consume more power doing so.

Oversampling architectures provide performance at par with the conventional designs by introducing subtle architectural changes-while digital functionality in CDR architectures offers flexibility in porting, post-fabrication testing, configuration and tuning. Often the digital control circuitry can be synthesized and laid out automatically since it is operating at a fraction of the input data rate. 


\subsection{Contributions from this work}

The contributions from this work are listed as follows:

- An understanding is developed using mathematical analysis and modeling as to why the oversampling CDR architectures progressively perform better with an increasing $O S R$ in the presence of random, deterministic and asymmetric jitter.

- A unified mathematical analysis is presented that binds all PD architectures using $O S R$ as a parameter. A system-level behavioral simulation flow emerges that incorporates the exact and generalized $n-x$ oversampling (NXO) PD equations derived in this work to simulate CDR performance in the presence of the given input jitter.

- An understanding develops of how the oversampling CDR architectures morph all the way from the BBPD architecture to the linear PD architecture and how the mathematics seems to have little choice but to behave.

- A novel all-digital Data Recovery (DR) architecture is implemented that establishes the data detection window using phases derived from a multi-phase oscillator. This imparts performance robustness over Process, Voltage and Temperature (PVT) corners while rendering the DR circuit rate-agile, adaptive and portable.

- Novel simulation methods are introduced to reduce the simulation time for transient jitter tolerance (JTOL) characterization. The idea is to exploit the maximum slope of the jitter sinusoid as this puts the most stress on the CDR tracking performance.

\subsection{Organization of the dissertation}

The seven chapters in the dissertation are organized as follows:

Chapter 1 introduces the dissertation and discusses the motivation that has propelled SerDes as a frequently demanded macro on SoC systems. Document organization is also explained. 
Chapter 2 reviews the essentials of SerDes systems and the role that CMOS technology has played in its evolution at the block diagram level. A short chapter on SerDes design cannot do justice to this topic.

Chapter 3 reviews the literature on CDR systems in detail and surveys the dominant and emergent themes that have been made possible due to the maturity of CMOS technology. The all-digital theme is emphasized.

Chapter 4 presents the unified analysis of PD architectures. The import of this analysis is two fold: The designer can find out how much oversampling ( $O S R)$ is enough for the type of input jitter present. Secondly, the exact equations can be used in a behavioral simulator to determine CDR performance.

Chapter 5 presents a novel oversampling digital DR architecture. The novel methods required to perform an efficient simulation of the architecture in the behavioral domain are presented. A Field-Programmable Gate Array (FPGA) implementation and measured results are presented.

Chapter 6 summarizes the work and lists the contributions.

Chapter 7 lists a subset of research possibilities.

References cited in this document appear at the end. The blue-colored hyperlinks facilitate navigation in the portable document format (pdf) file for this dissertation that is available through search engines on the internet. 


\subsection{Chapter overview}

This chapter briefly explains the SerDes hierarchy and subsystem interconnections using block diagrams. Important concepts, definitions and specifications from the SerDes parlance are also introduced. It is important to know the environment in which a CDR circuit operates as it is an essential subsystem of the SerDes macro. This chapter is an attempt to abbreviate the explanations that follow in later chapters. The experienced SerDes engineer can skip this chapter without loss of continuity.

\subsection{Basic high speed serial link}

A serial link [9] consists of a transmitter (TX), a channel (CX) and a receiver (RX) as shown in Figure 2.1.

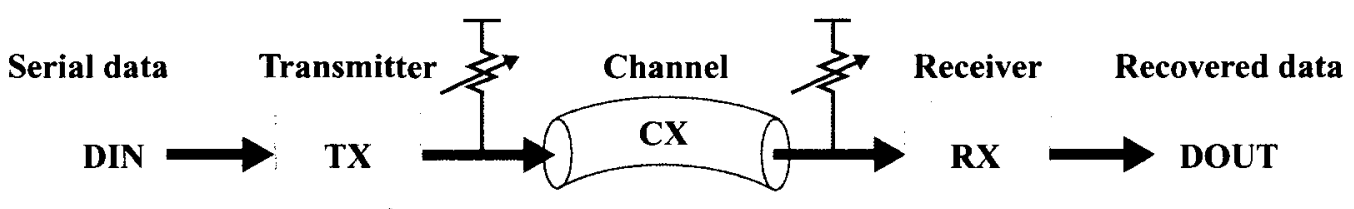

High-Speed Serial Link

Figure 2.1 Serial link overview

What makes it an HSSL [52][59] is a notion that cannot be discussed in isolation from the underlying CX. The symbol transfer speed is measured in bits per second (bps). 
In 1980s, a few kbps would have been characterized as high speed, but as of 1990s a capacity of few Gbps had been reached. Aggregate SerDes bandwidths have, as of 2010, reached 2.4 Tbps on CPUs using a host of advanced techniques [68][69]. For the long-haul optical market, several hundred Tbps is the ambition for continent-wide deployments [70]. Optical links use optical hardware, monomode fiber optic cables and scalable Dense Wavelength Division Multiplexing (DWDM) techniques [156]. New materials and substrates have to be engineered continuously to cope with ever-demanding HSSL requirements.

\subsection{System Synchronization}

A timing protocol needs to be agreed upon between sender and receiver if information is to be shared. This classification is briefly explained in Table 2.1 [6][11].

TABLE 2.1: SerDes clocking schemes

\begin{tabular}{|l|l|l|}
\hline Clocking Mode & \multicolumn{1}{|c|}{ Description } & \multicolumn{1}{|c|}{ Comments } \\
\hline Synchronous & $\begin{array}{l}\text { Same clock for the } \\
\text { source and destination }\end{array}$ & $\begin{array}{l}\text { Known frequency, some clock skew; Not feasible for multi- } \\
\text { drop architectures; feasible for short point-to-point on-chip } \\
\text { applications or very low-speed PCB systems }\end{array}$ \\
\hline Asynchronous & $\begin{array}{l}\text { Request / Acknowledge } \\
\text { signals in a handshake } \\
\text { mode }\end{array}$ & $\begin{array}{l}\text { Too slow for modern data rates and backplane communica- } \\
\text { tions but with notable exceptions [83]. }\end{array}$ \\
\hline Mesochronous & $\begin{array}{l}\text { Same clock for source } \\
\text { and destination, but with } \\
\text { a different phase with } \\
\text { bounded variations }\end{array}$ & $\begin{array}{l}\text { Wastes power in the transmitted clock signal, a phase align- } \\
\text { ment mechanism is necessary on the RX side. Enough edges } \\
\text { need to be present to ensure recovery. A CDR circuit is not } \\
\text { required. Used for memory and DMA chipsets. }\end{array}$ \\
\hline Plesiochronous & $\begin{array}{l}\text { Independent clock } \\
\text { sources for source and } \\
\text { destination }\end{array}$ & $\begin{array}{l}\text { Has to acquire/track the frequency of the clock signal embed- } \\
\text { ded in data; Saves power by not transmitting the clock; feasi- } \\
\text { ble for Gbps data rates over FR-4 backplanes; feasible for } \\
\text { multi-drop scenarios as well. A CDR circuit is required to } \\
\text { establish a sampling point following a DFE. }\end{array}$ \\
\hline Heterochronous & $\begin{array}{l}\text { Nominally at different } \\
\text { frequencies }\end{array}$ & $\begin{array}{l}\text { Integral path of the CDR circuit brings the oscillator in line } \\
\text { with the incoming symbol rate for multi-protocol SerDes sys- } \\
\text { tems upon a protocol change request such that the plesiochro- } \\
\text { nous case applies thereafter. }\end{array}$ \\
\hline
\end{tabular}




\subsection{The SerDes usage overview}

The selection of the package is the first and foremost design decision for systemon-chip (SoC) macros [10]. As SoCs have become dense, a portion of the functionality has migrated on to the package from the PCB [81]. This is shown in Figure 2.2 where a multilayered substrate within the package is used to route signals to the flip chip. With such complexity to handle, package modeling [73], system-level pin assignment, macro placement, floorplanning [76], test boards, ATE test time [74], electromagnetic interference (EMI) compliance [75], IP integration and validation [80], heat dissipation [139], mechanical stability and package cost considerations are not afterthoughts, but mandatory planning stage questions. With the system buses increasing in width, and thermal hot spots deteriorating chip lifetimes [79], a SerDes emerges as the commercially viable solution for enhanced SoC functionality.

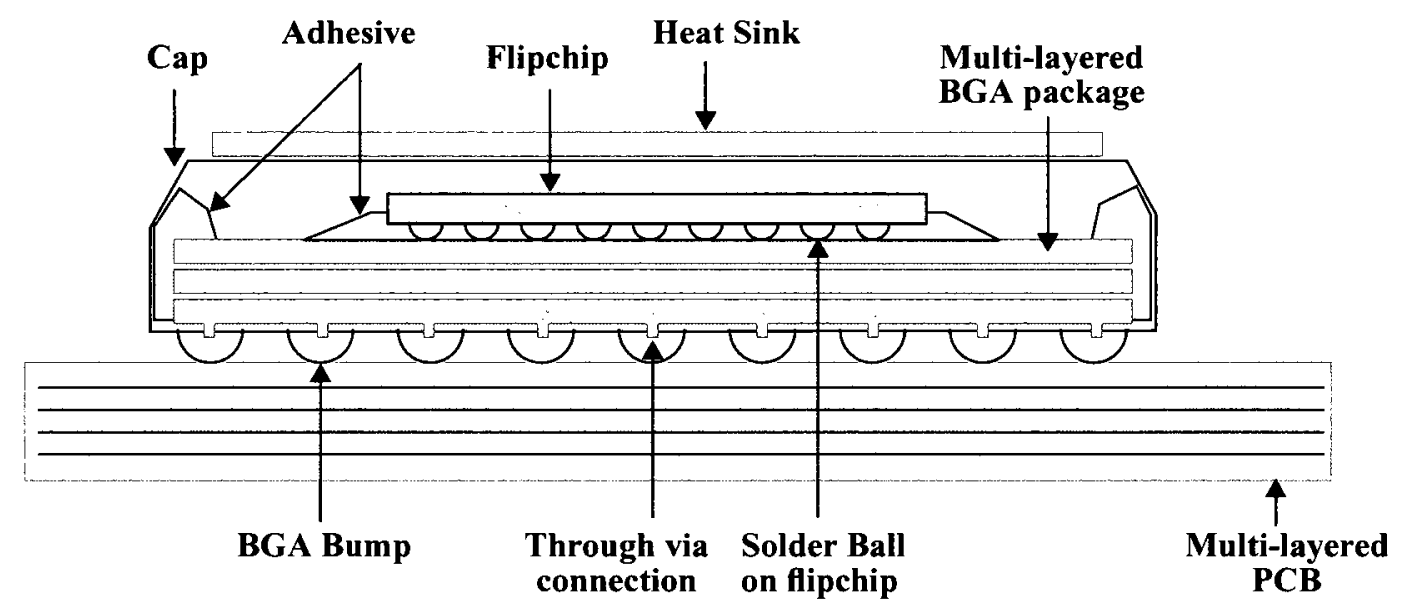

Figure 2.2 Flip chip die on a BGA package (cross section)

This backdrop drives the SerDes design in addition to the expedient design flow, technology and node selection, architectures, platforms, modeling tools [72], clocking schemes [6], calibration algorithms etc. Many of these decisions involved in SerDes design are recursive and cannot be resolved in isolation. The way the SerDes macros are arranged on-chip results in one or two dominant crosstalk aggressors, since pad dimen- 
sions and layout prevent many pads in close proximity. The EMI compliance is also improved due to a lower number of high-current switching outputs on-chip or on the PCB. Fewer pins are required as well. Hence, SerDes macros have emerged as cost-effective solutions for a host of challenges associated with complex SoCs. An example showing the usage of multiple SoC chips on a multi-layered PCB appears in Figure 2.3. To minimize the length of PCB traces, SerDes macros are often placed near the edge of the flip-chip die. Power and ground planes on the PCB provide short and numerous connections to the ball-grid array (BGA) package in order to improve power and signal integrity.

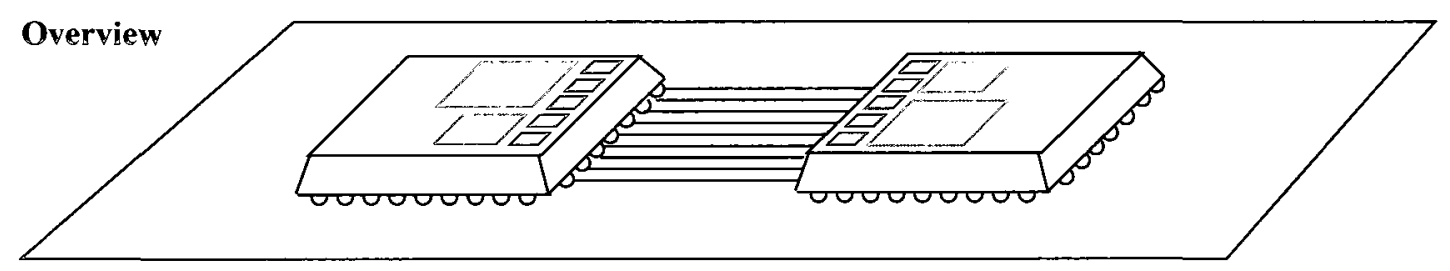

\section{Cross Section}

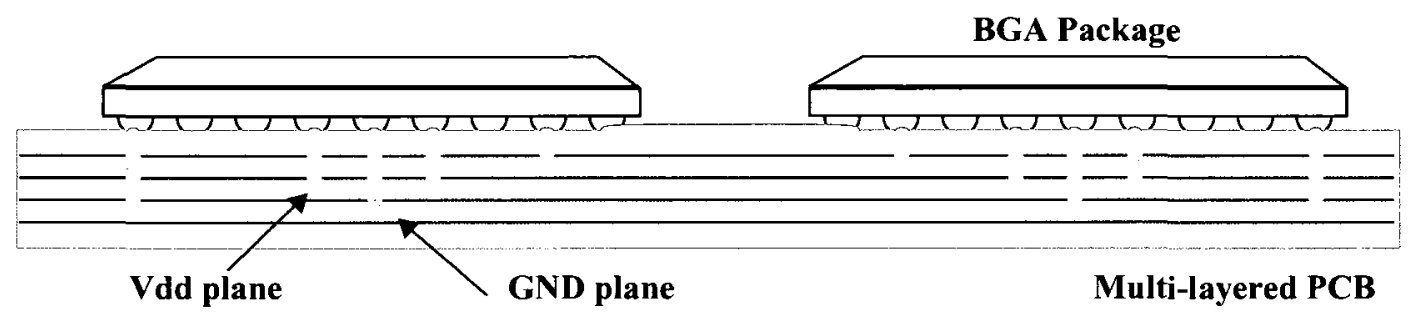

Figure 2.3 Typical off-chip SerDes usage

BGA packages with a generous number of pins are the norm on most SoC chips. The close proximity of the flip chip pads and the circuits reduces power supply resistance and helps achieve compliance over PVT corners. The BGA bumps have much lower inductance compared to the vintage flat package bondwires resulting in reduced reflections and shorter interconnect delays.

Typical SerDes macro usage within a complex chip is shown in Figure 2.4 [88]. A SerDes macro is marketed as a receiver/transmitter/clock generator (RX/TX/CG) combo with essential control signals included in the description. This facilitates the integration of the SerDes IP macro with the inhouse SoC project. A complex macro can have multiple 
RX/TX pairs to increase the aggregate bandwidth while the lanes typically share the CG block [69]. Compatibility between various SerDes IP vendors is virtually non-existent and requires a much needed standardization initiative.

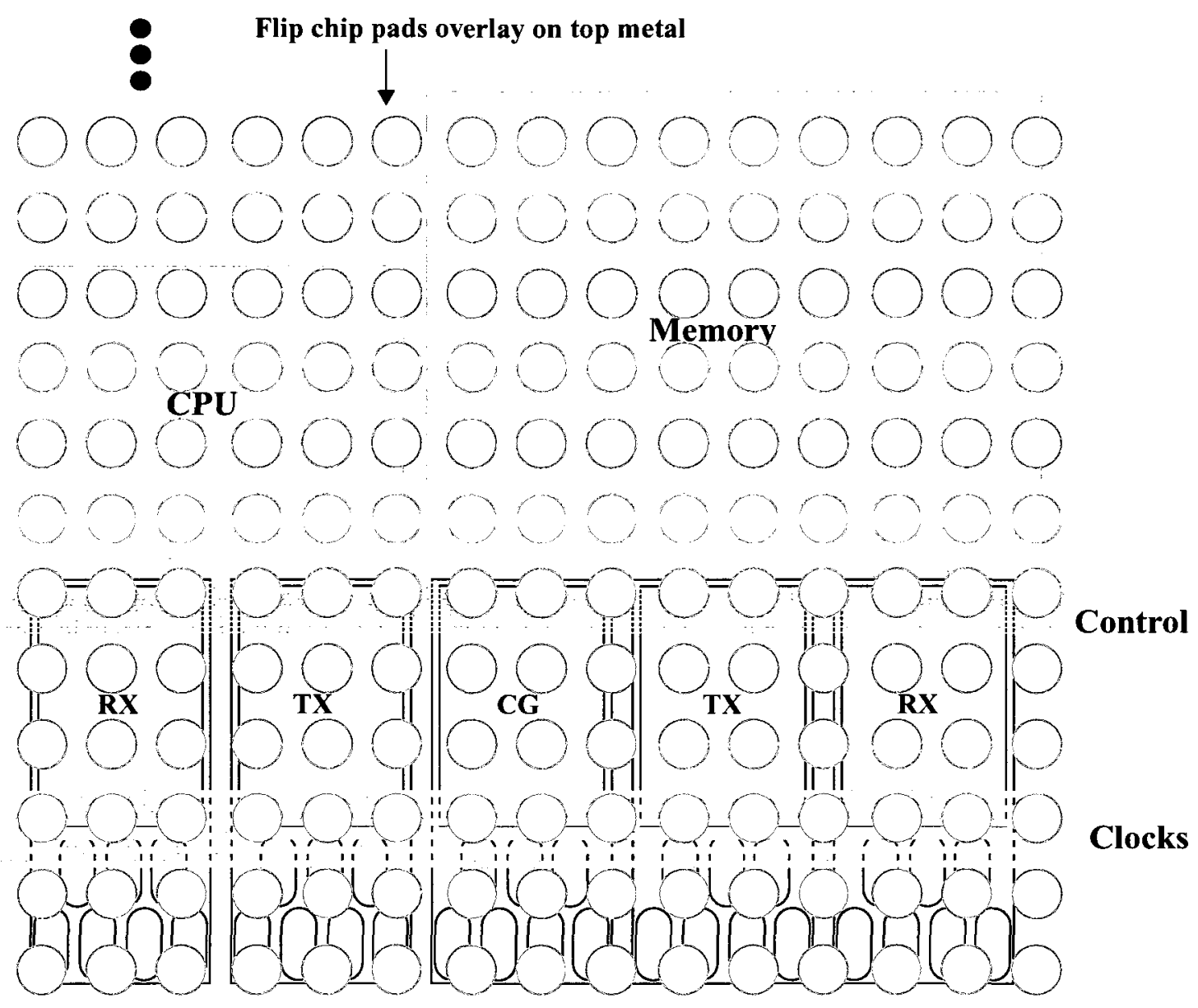

Figure 2.4 Typical SerDes Usage in SoC modules

Figure 2.5(a) shows an example of parallel data transfer on-chip from between two block buffers situated at the ends of a complex SoC. The arrangement quickly becomes pad limited wasting silicon area. Due to multiple buffers and biasing arrangements, power requirements also increase drastically and EMI compliance is harder to achieve. The individual bit drivers in the parallel case consume power depending on the net capacitance per unit length in order to maintain error free communication. Crosstalk is increased and 
phase aligners are required at both ends. Additional supply filtering has to be employed to improve power integrity.

Buffer_A

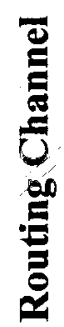

DSP

Buffer_B
Drivers Buffer_A

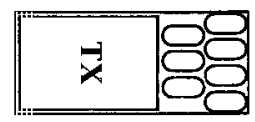

ד्र

Core_A

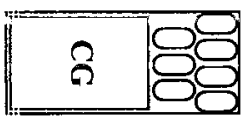

DSP

Core_B

Drivers Buffer_B
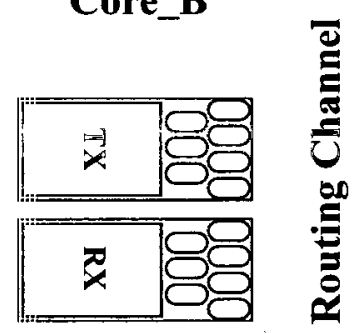

(a) Parallel

(b) SerDes

Figure 2.5 On-chip data transfer (a) Parallel vs. (b) SerDes

Using an on-chip SerDes macro as shown in Figure 2.5(b), however, more circuitry can be fitted in the same area and power budget [87]. The EMI compliance is also improved due to the reduction of power hungry drivers.

The software written for serially interfaced systems remains transparent to the data rate [71] and can be substantially reused. This software reuse is more cost-effective than the parallel scenario where a simple change from 32-bit bus to a 64-bit bus necessitates a re-design of the entire software and hardware platform. As the technology node scales, the 
possibility of the reuse of test beds, digital IPs and system software in the SerDes macro provides avenues to streamline product revisions while reducing the final cost.

\subsection{Channel}

The data rate dictates the substrate for the channel. The design techniques for TX, $\mathrm{RX}$ and modeling techniques for the $\mathrm{CX}$ are largely dependent on the input data rate.

TABLE 2.2: Channel types and data rates

\begin{tabular}{|c|c|c|}
\hline Channel & Data Rate & Remarks \\
\hline Satellite & few $\mathrm{Mb} / \mathrm{s}$ & $\begin{array}{l}\text { Used for transcontinental and tactical communications; Specialized } \\
\text { links with unique considerations for distortion created by atmo- } \\
\text { spheric absorption and noise. Expensive; used for TV broadcasting. }\end{array}$ \\
\hline Fibre optic cable & several $100 \mathrm{Tbps}$ & $\begin{array}{l}\text { Used for long haul applications over hundreds of kilometres. Repeat- } \\
\text { ers are required. Jitter peaking is to be avoided (SONET). Optical } \\
\text { leakage (crosstalk) and multi-modal transport are important issues } \\
\text { causing ISI. Interface with electrical hardware requires expensive } \\
\text { optical components. }\end{array}$ \\
\hline FR-4 PCB & $\sim$ upto 20 Gbps & $\begin{array}{l}\text { Used for communication and server backplanes, DFE is required for } \\
\text { most deployments due to severe attenuation and ISI distortion } \\
\text { acquired through the channel. PAM- } 2 \text { and PAM-4 links have been } \\
\text { widely reported. } 20 \text { Gbps techniques are recent, } 10 \mathrm{Gbps} \text { is the norm. } \\
\text { Phase aligner may be required instead of a CDR ciruit for shorter } \\
\text { links. }\end{array}$ \\
\hline Ethernet cable & upto 1.25 Gbps & $\begin{array}{l}\text { Gigabit ethernet is now in wide use in the PC market. Crosstalk from } \\
\text { other PC circuits is a major concern. DFE and crosstalk cancellers are } \\
\text { required. Interesting applications have surfaced for audio/video dis- } \\
\text { tribution over short distances using the GbE platform. }\end{array}$ \\
\hline Coaxial cable & upto 3 Gbps & $\begin{array}{l}\text { Short-haul, few } \mathrm{km} \text {; a linear equalizer is required as a minimum. to } \\
\text { restore amplitude }\end{array}$ \\
\hline $\mathrm{Cu}$ traces & upto $20 \mathrm{Gbps}$ & $\begin{array}{l}\text { on-chip communications; phase aligner required along with forward } \\
\text { equalizer. Cross-talk is a concern. }\end{array}$ \\
\hline $\mathrm{Al}$ interconnect & $\sim$ upto $10 \mathrm{Gbps}$ & $\begin{array}{l}\text { On-chip data transfer in older technology nodes earlier than } 45 \mathrm{~nm} \\
\text { using the thick top Al metal }\end{array}$ \\
\hline Wireless & $<1 \mathrm{Gbps}$ & $\begin{array}{l}\text { Using MIMO receivers that are computation-intensive. For indoor } \\
\text { applications requiring portability. Attenuations and reflections from } \\
\text { moving objects; changing environment. }\end{array}$ \\
\hline
\end{tabular}


The long-haul fiber-optic systems have bandwidths that cannot be handled by semiconductor chips. Optical hardware such as laser modulators, erbium-doped fiber amplifiers, optical mux/demux and PIN photodiodes are used to demultiplex optical traffic down to $40 \mathrm{~Gb} / \mathrm{s}$. Subsequently, the data is handled by III-V substrates such as GaAs or other expensive processes [129][132][131]. Modern CMOS processes are aptly suited for lower bit rates $(10 \mathrm{~Gb} / \mathrm{s}$ or below). The MUX/DEMUX arrangements can increase the throughput of the silicon chips to hundreds of $\mathrm{Gb} / \mathrm{s}$ using multiple data lanes [69].

Satellite, optical and wireless links are outside the scope of this work. Wireless interconnects are currently restricted to less than $1 \mathrm{~Gb} / \mathrm{s}$ using Multiple-Input MultipleOutput (MIMO) deployments. All these channels have to deal with noise, crosstalk (or optical leakage) and ISI (or multi-modal transmission) that are the major challenges.

\subsubsection{Modeling the channel}

Channel modeling continues to be a research topic [67][82][78][87]. A typical FR4 backplane channel is shown in Figure 2.6.

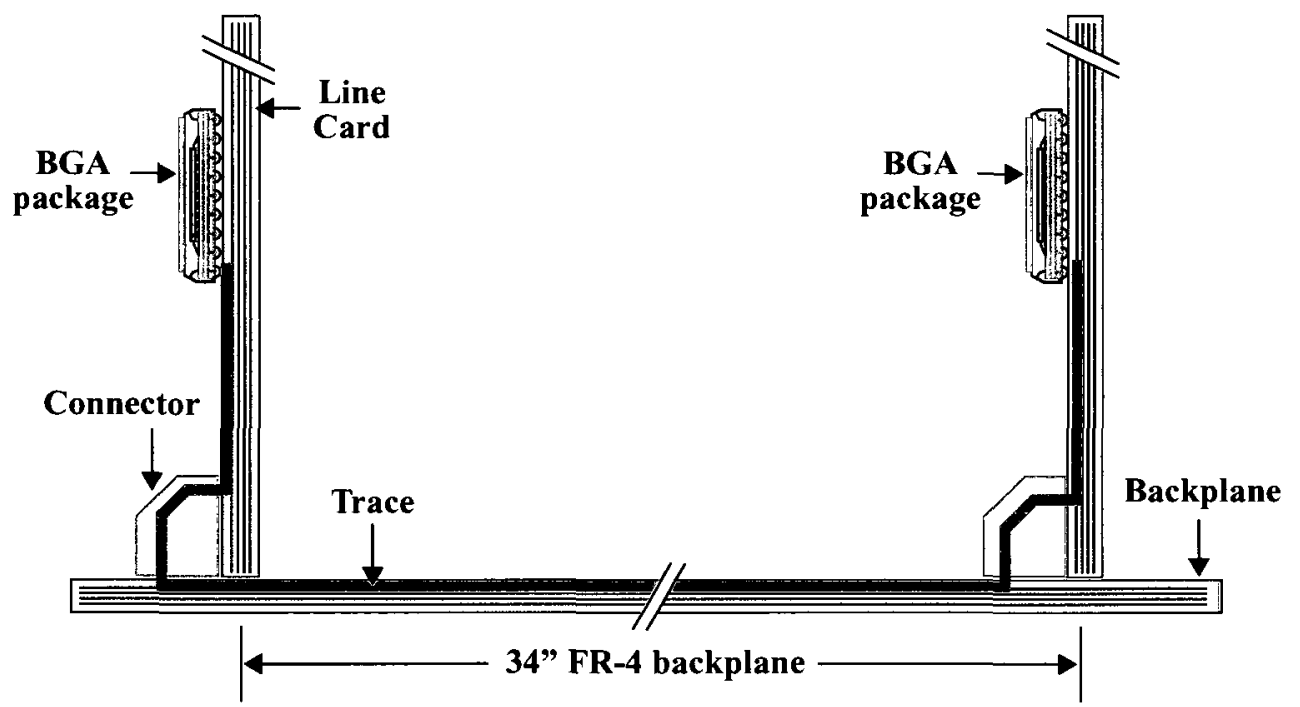

Figure 2.6 A typical FR-4 backplane channel

This is the most common wireline SerDes channel. The standard backplane trace length is 34 ". The line cards serve to add roughly 7" of trace length. A considerable num- 
ber of connector vias and material transitions increase reflections. It is not possible to know the precise channel at start up; although a rough estimate can be made. After installation, a more modern line card might be substituted for an older one or vice versa. The trace length for a multi-drop scenario is different for the same line cards-and so is the near-end crosstalk (NEXT) and the far-end crosstalk (FEXT). From start up to normal operation, the temperature changes before stabilizing. Local line cards might introduce varying signal integrity situations depending on the function of the card. With these factors in mind, adaptive DFEs have become the norm for FR-4 backplanes [85]. Additionally, several parametric models (from best-case to worst-case) are used to achieve an estimate of link performance at design time. Standard channel models are provided by manufacturers for FR-4 backplanes. Channel models are derived using s-parameters or time domain reflectometry (TDR) techniques [82]. A vector network analyzer is used for measuring the multi-port s-parameters and the results are saved to a standard file that works with industry standard simulators. For channel modeling using TDR techniques, a sampling scope with a TDR attachment is required.

\subsection{The SerDes itself}

The parallel input data from the SoC chip is serialized using a parallel-in, serialout (PISO) module before a transmitter is employed. This is shown in Figure 2.7. A SerDes is sometimes referred to as a HSSL but the meaning is clear from the context.

In order to guarantee a minimum transition density, data are encoded if the specifications require it-e.g., 8B/10B encoding converts an 8-bit payload to a 10-bit payload and guarantees a transition every five bits-which is quite similar to a 9-bit PRBS. With conventional circuits, a long run of zeros or ones either saturates the receiver or unlocks the conventional CDR system due to CP leakage. The data is deserialized after reception and decoded. For wireless links, a modulator is employed before the data can be sent and 
received at all using air waves. The important modules of this SerDes arrangement are briefly explored in the following subsections.

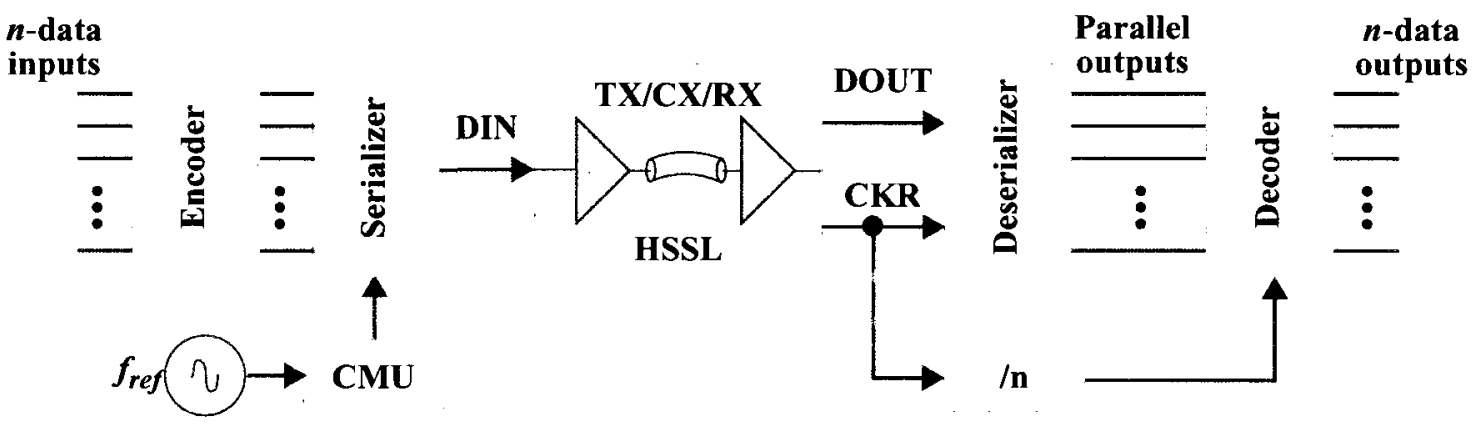

Figure 2.7 SerDes block diagram

\subsubsection{Transmitter}

The most prevalent technique in transmitter design is the pre-emphasis of the transmitted bits using a pre-emphasis filter [112]. Figure 2.8 shows a 3 -tap feed-forward equalizer scheme as it is implemented in a typical transmitter.

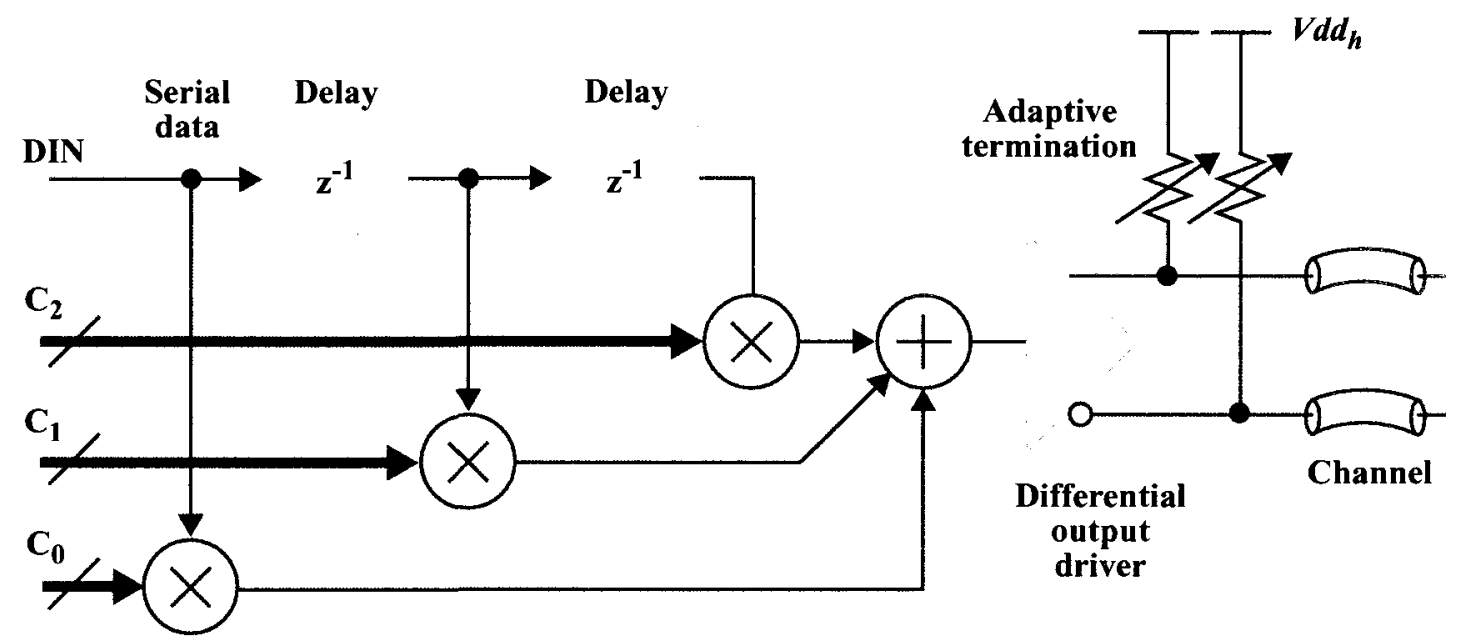

TX

Figure 2.8 3-tap Pre-emphasis block diagram

The need for the pre-emphasis filter arises since the channel causes disproportionate attenuation of the high-frequency content in the data. The reasoning goes that if the 
high frequency content is accentuated prior to transmission, then after the attenuation and distortion caused by the channel, the eye remains usable at the RX front-end without a significant performance penalty. The flip side of the argument is that it degrades the NEXT and FEXT in addition to putting stress on the output transistors. The EMI compliance of the product can also suffer. The multiply and add structure can be replicated in circuit form using a current output steering Digital-to-Analog Converter (DAC). It is separately discussed later in the chapter.

\subsubsection{Data phase aligner}

For source synchronous systems, the clock can be positioned at the optimum sampling point by choosing a clock phase from either a cell-based delay line or a multi-phase oscillator. This is conceptually shown in Figure 2.9.

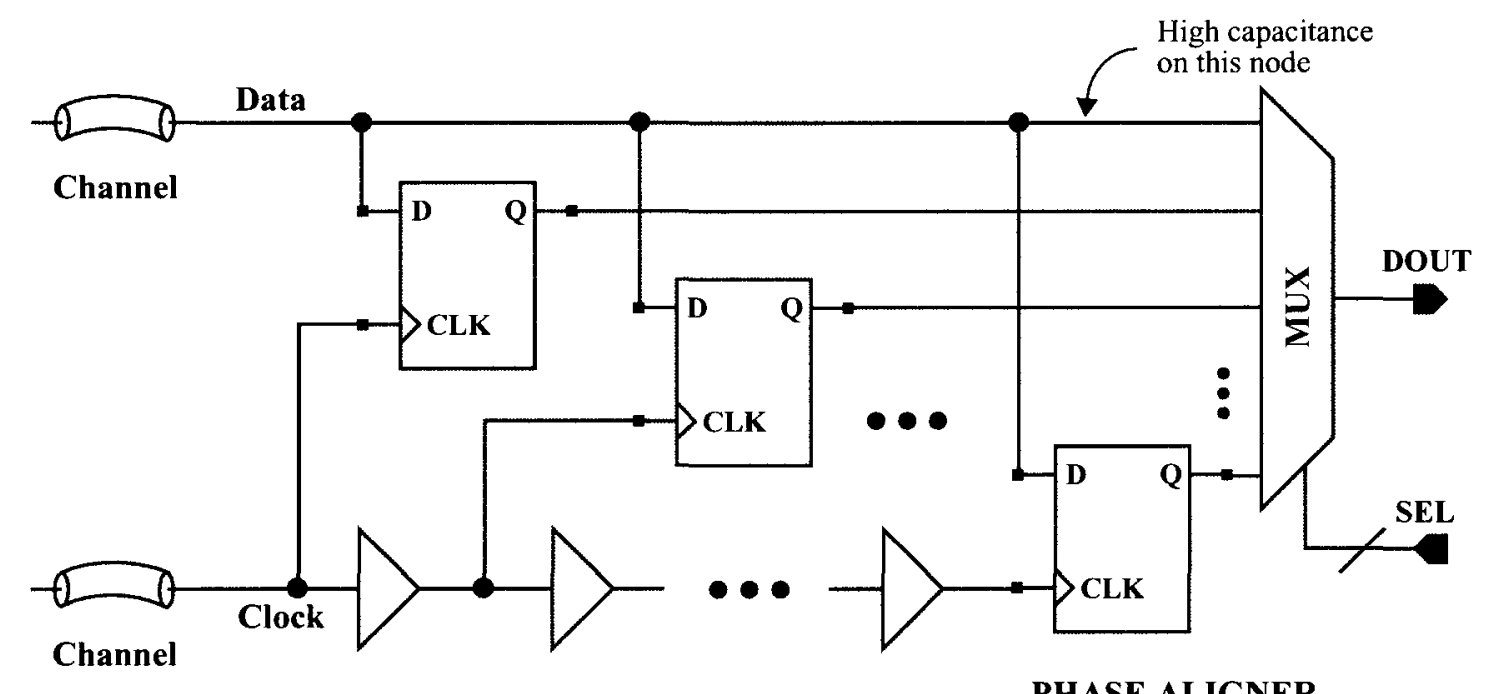

PHASE ALIGNER

Figure 2.9 Data phase aligner

The number of storage elements increase the capacitance on the 'Data' node and hence limit the range of correction. Adding further flipflops would require a DFE to restore the amplitude of the input signal. The delay cells need PVT compensators for accurate results. The propagation delay through various MUX inputs has uncorrelated 
variations. A DLL can be used instead of an open-loop delay line but it adds complexity and power. The DLL loop filter requires a large area as well.

\subsubsection{Decision Feedback Equalizers}

The essential function of the Decision Feedback Equalizer (DFE) [86][85] is the opening of the vertical eye at the RX prior to sampling by the recovered clock. The recovered clock is provided by the CDR circuit that places the sampling point at the centre of the eye thus improving the horizontal eye. CDR architectures are studied in detail in Chapter 5. To avoid duplication, only the interconnections are emphasized here.

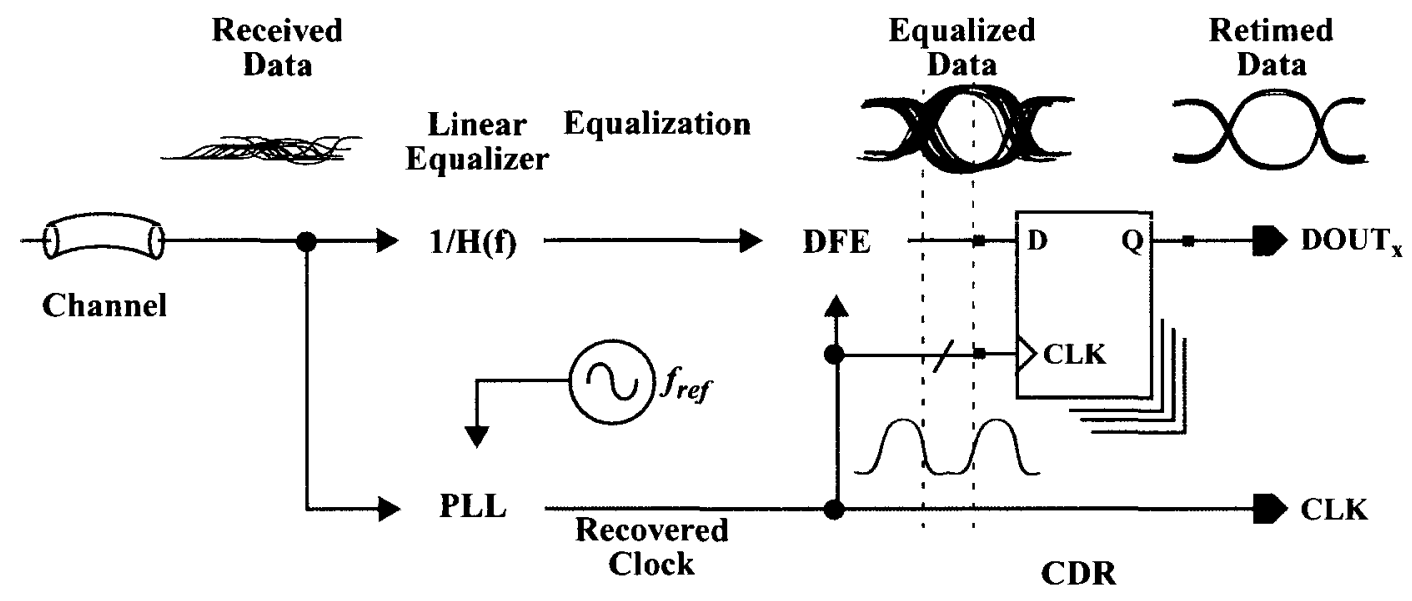

Figure 2.10 DFE and CDR interconnections in RX

The block diagram of a typical DFE is shown in Figure 2.11. The AGC circuitry is followed by the forward equalizer (FEQ) that compensates for the channel low-pass characteristics. The data slicer has a threshold of zero. The summing node is a CML type current summing circuit that needs to have a high bandwidth in order to maintain fast transitions times that improve the BER. If too many feedforward and feedback taps are used, then the large devices reduce the bandwidth of the summing node. Hence, there is a trade-off. The adaptation engine looks at the error slicer output and decimates the errors to avoid instability by updating taps too often. The AGC tap is used to keep the vertical eye at the error slicer threshold. The tap weights are controlled by the multi-bit digital outputs 
$\left(t_{-m}, \ldots, t_{0}, \ldots, t_{n}\right)$ from the LMS adaptation engine. Using capacitors to store the tap value is not very effective, since the LMS update happens at a frequency of a few $\mathrm{kHz}$ (maximum) and the capacitors can discharge significantly during this period causing a systematic ripple at LMS update frequency causing a reduced vertical eye opening that defeats the original purpose of the DFE.

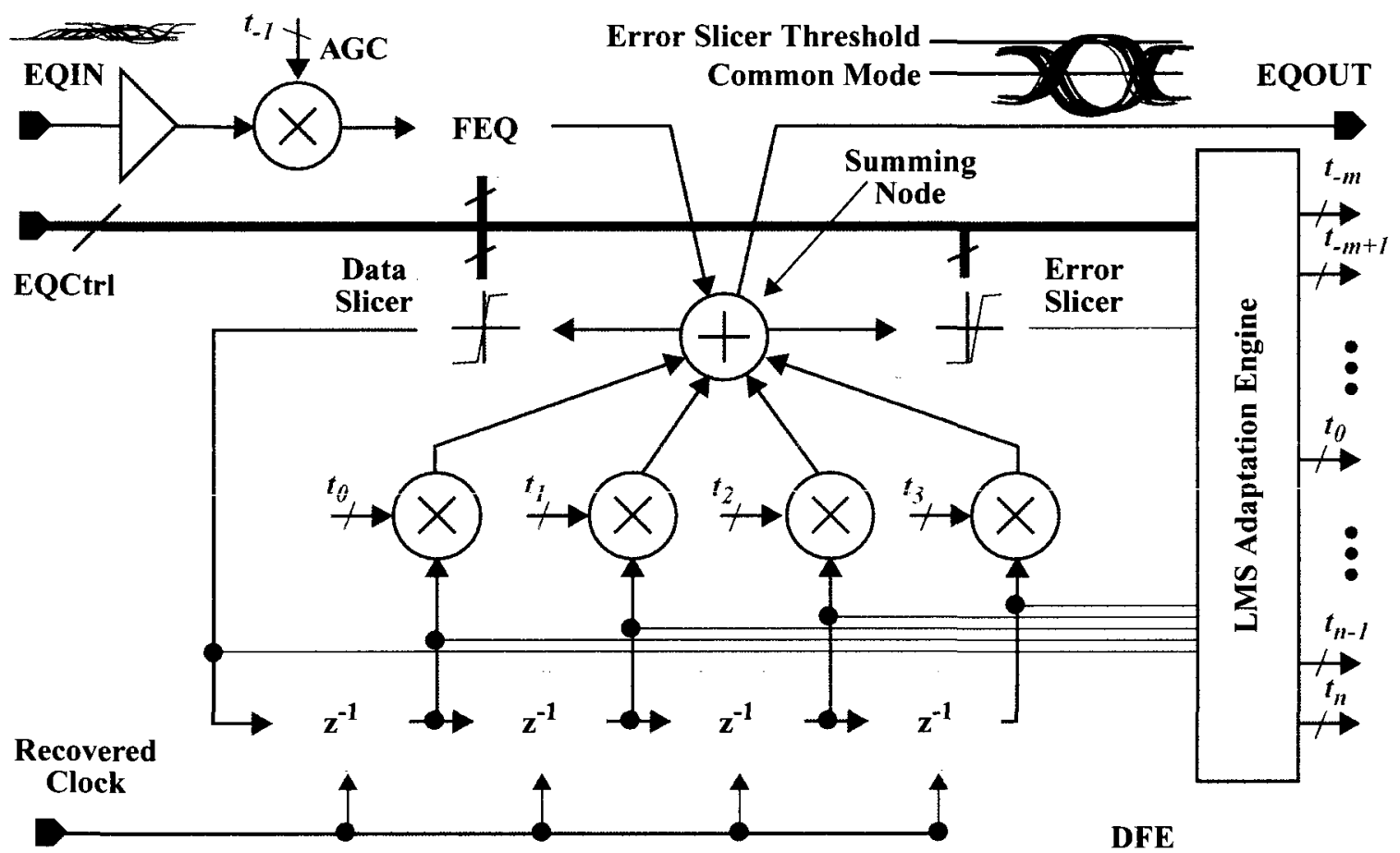

Figure 2.11 DFE block diagram

The control bus performs many functions that are not explicitly shown in Figure 2.11. It provides control signals to observe analog nodes. The ability to bypass the LMS adaptation engine during debugging, training, and calibration modes is also an essential part of the design process. The PVT calibration for dummy branch at the summing node, common mode trimming and settings for the thresholds for the data and error slicers is also managed with the control bus by using compensated DACs. Commercial receivers are complex systems.

The removal of ISI is achieved by subtracting the effect of the past symbols called 'post cursors' from the current symbol (called the 'cursor'). The pre-cursor is similar to 
watching the future and preparing in advance for it by confining the energy in the current symbol from spreading into adjacent symbols. A judicious selection of FEQ parameters under program control can reduce the number of taps for certain types of channels thus improving the BER.

\subsubsection{Current output Digital-to-Analog Converter (iDAC)}

Multiplying a digital bit by a weighted value and adding the result is a common sub-function in mixed signal implementations. Figure 2.12 shows an example of the circuit implementation of an iDAC circuit.
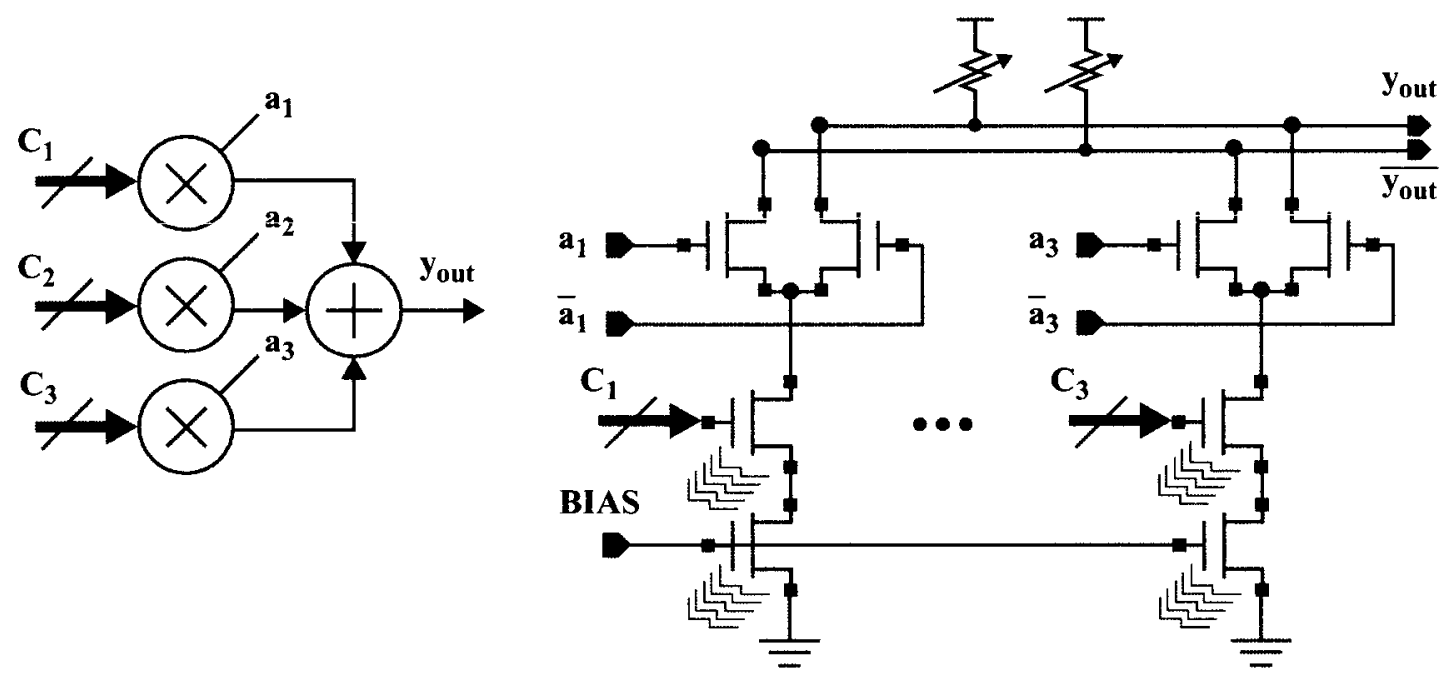

(a) iDAC symbolic

(b) iDAC circuit

Figure 2.12 Current output DAC

The bandwidth of the summing node determines the transition times at the output and hence the quality of the eye opening. An adaptive termination is the norm on multiprotocol SerDes implementations as an aid to post-fabrication tuning. For subtracting the effect of a certain bit, the polarity of the data inputs can be flipped. The transistors in the mirror as well as the switches can be implemented as unit-sized or binary-weighted depending on the required accuracy. For improved result over corners and due to linearity considerations, the current density in the switches and the biasing transistors should match 
well. If this multiplication is being performed at a low frequency, then the active bit and control signals can be combined to provide more headroom for the manipulation of output voltage swing.

\subsubsection{Pseudorandom bit sequence (PRBS) generators}

For BIST purposes, a Linear Feedback Shift Register (LFSR) is integrated on macros. The LFSR generates a pseudo-random binary sequence (PRBS). A MUX can be used to switch between incoming data (in normal mode) or the PRBS (in testing or training mode) as required. The LFSR can be arranged in the Galois or the Fibonacci configuration as shown in Figure 2.13.
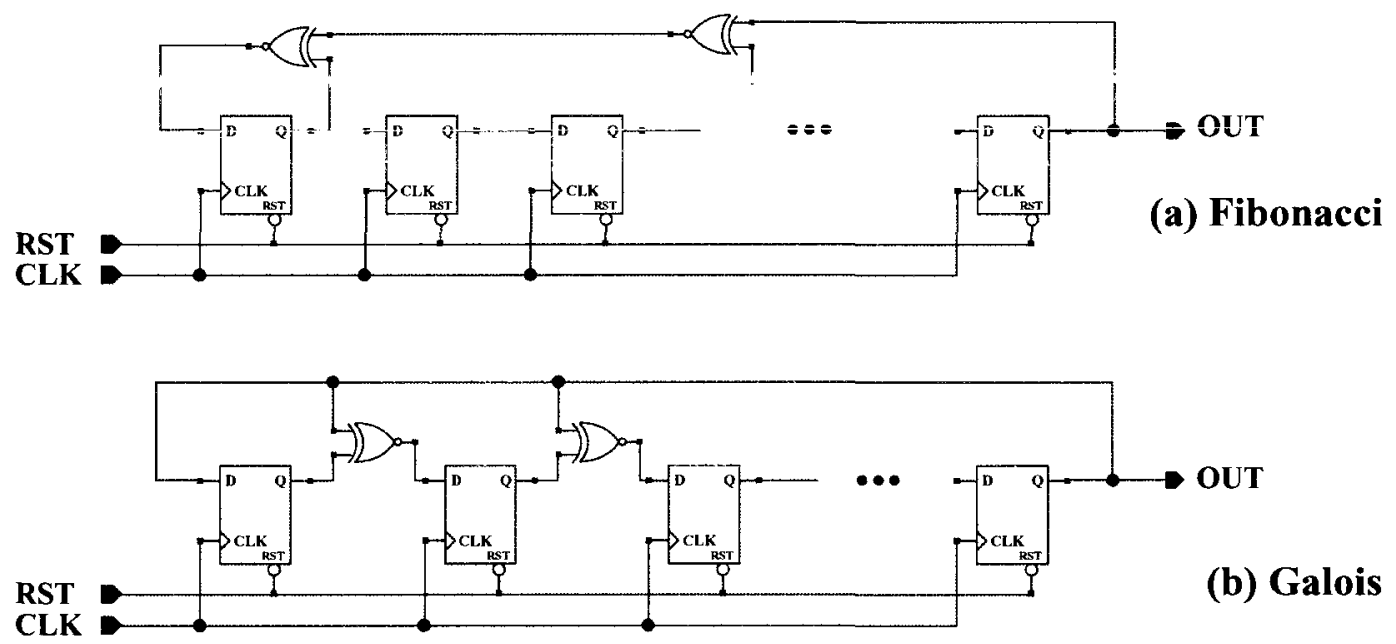

(b)

Figure 2.13 LFSR configurations

These implementations differ in the placement of the XOR gates. For high speed SerDes, the Galois configuration provides a distinct speed advantage since the feedback delay in the loop is much smaller compared to the Fibonacci configuration. Depending on the length of the polynomial, a suitable feedback connection is provided to the XOR/ XNOR gate to generate a maximal length sequence. Figure 2.14 shows a Fibonacci LFSR macro. At the application of the reset (RST) signal, the LFSR should not lock in a state at start-up that it cannot escape. Thus the maximal PRBS length is $2^{n}-1$, where $n$ is the 
number of storage elements. The external connection in the feedback path makes the PRBS generator more modular for later use as a pattern generator as well as a checker. In order to generate a PRBS using multiple PRBS running at slower speed, a mux can be used.

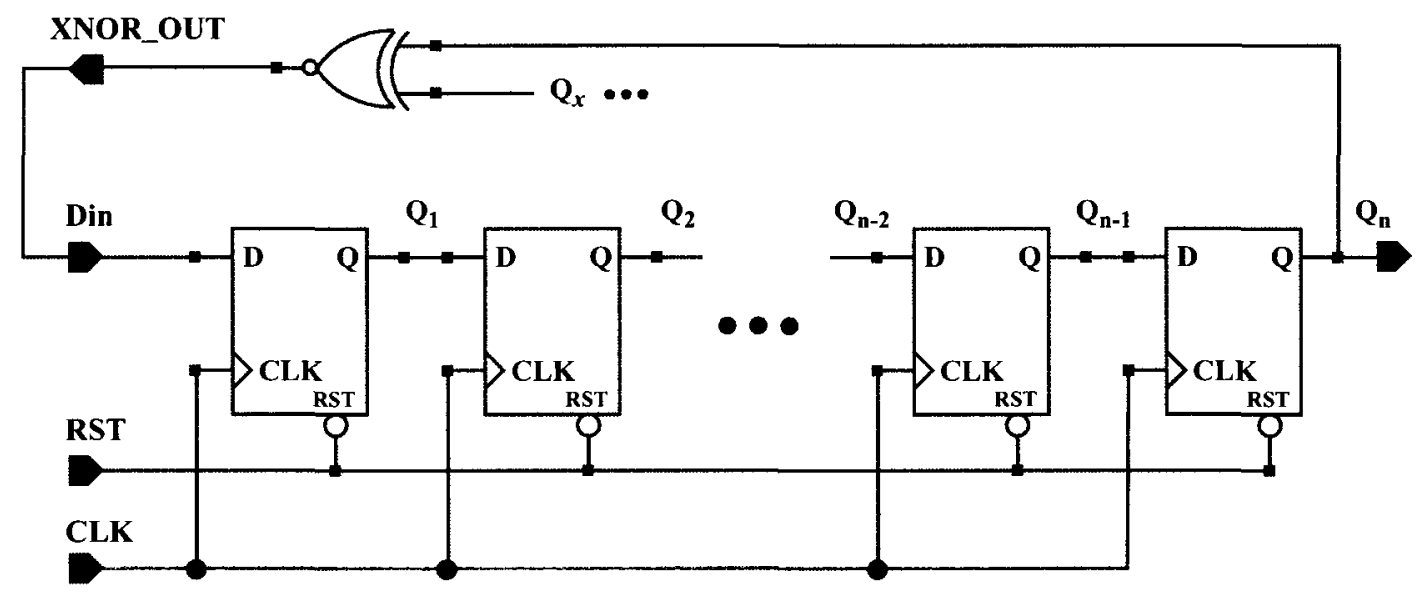

Figure 2.14 PRBS generator block diagram

\subsection{Definitions and specifications for SerDes}

Definitions emerge so people can communicate. Specifications exist to ensure compliance between products and systems. SerDes specifications can be found in product datasheets - and the specifications for the CDR circuit within a SerDes macro are not cited separately. In order to limit the scope of this discussion, the specifications for CDR circuits will be discussed after a review of CDR architectures in Chapter 3.

\subsection{Choice of technology}

SerDes is a building block for larger SoC systems and most SoC systems are fabricated in CMOS technologies. Thus the most common substrate for a SerDes IP macro is the CMOS technology. A coherent discussion of analog design trade-offs can be found in classical textbooks [1][3][4][5]. Moore's law still applies to the CMOS transistor density, but not to the speed and throughput for CPUs. Although Hardware Description Language 
(HDL)-driven automated synthesis and layout tools enable astounding automation and reuse, the sardine-can approach has hit interconnect and thermal barriers and the hunt is on for novel substrates and techniques. Reduced complexity architectures employing parallel functionality that provide increased throughput are being actively adopted.

\subsubsection{Why less analog is better}

The implications of scaling for analog design are dire. Many interacting specifications across different dimensions need to be recursively optimized before a product can be integrated with a SoC chip. This is pictorially illustrated in Figure 2.15.

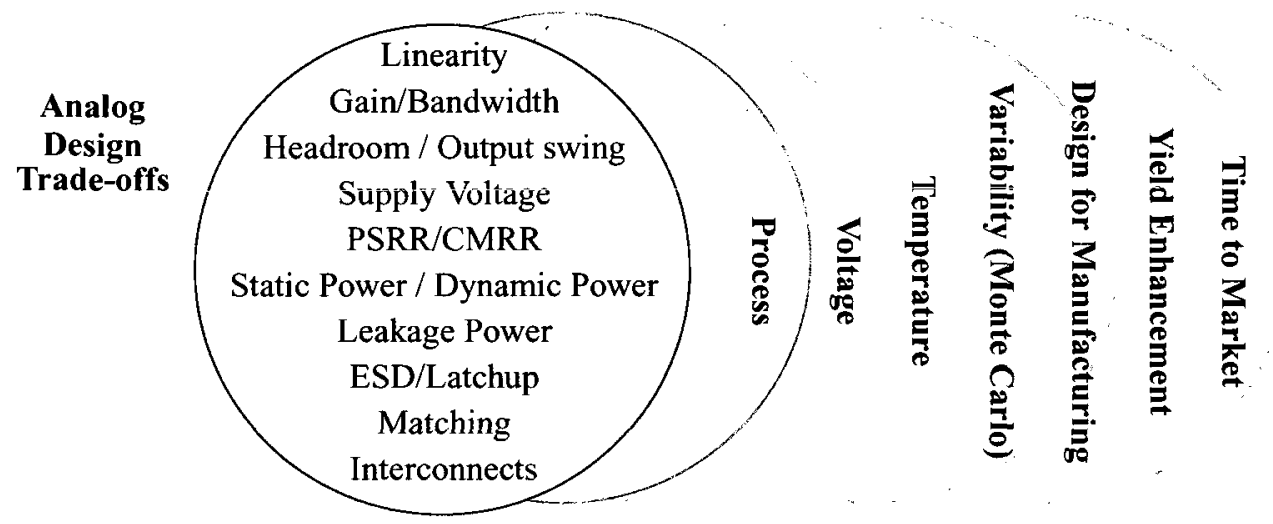

Figure 2.15 Analog design trade-offs across many dimensions

Human beings do not think in many dimensions at the same time- or do so very poorly without advanced simulators. The accuracy of simulation depends on the models provided by human knowledge and models cannot be upgraded without educated feedback. This implies that analog circuit design cannot be decoupled from the fabrication facility as long as models change regularly and this results in a number of open loop situations. Timely completion of an analog-intensive project is seldom guaranteed. SerDes design is a complex AMS task that requires knowledge of the past mishaps as well as intensive simulation resources. Less analog complexity is the natural choice. Experienced designers know that there is no such thing as an all-digital circuit let alone an all-digital 
system. At high speeds, even the simplest of digital gates become analog circuits where transition times, delays, fanouts and output impedances matter. The mixed-signal nature of the SerDes macro prevents such a trivial simplification of nomenclature across the board. To reduce the time-to-market and ensure first-pass success, digital IP blocks are introduced in favor of analog blocks wherever possible since HDL descriptions can be seamlessly ported to advanced process nodes.

\subsection{New directions in SerDes systems}

SerDes architectures are constantly evolving due to technological progress and the ever-increasing throughput requirements. This section reviews the relatively newer trends in SerDes design.

\subsubsection{Wireless inter-chip SerDes architectures}

Wireless techniques for short-range SerDes scenarios have gained considerable ground since the turn of the millennium [123]. Not all substrates are suitable for all systems. For instance, memory circuits utilize specially tailored processes; front-ends require low-noise amplifiers whereas the transmitters are fabricated using III-V processes capable of delivering higher currents. The ability to move information between different types of circuits, e.g., CPU and memory, without wasting power in buffers is commercially important. A notable new direction in this regard is the wireless inter-chip SerDes to transmit data between stacked dies in a Systems-in-Package (SiP) scenario. The TX/RX strategies for communication between stacked chips utilize capacitive, inductive, micro-bump and Through-Si via (TSV) techniques [124]. A new parameter that becomes important in such stacked-chip studies is the thickness of the die itself. Better magnetic field scaling is expected with thinner wafers so this opens up the possibility for further research on fabrication, stacking and packaging. A $1 \mathrm{~Tb} / \mathrm{s}$ throughput wireless SerDes has been presented in the literature [125][126] with an area of $2 \mathrm{~mm}^{2}$ and $3 \mathrm{pJ} / \mathrm{bit}$ power requirement. This 
direction of technological development is profound as it opens the doors to communication between stacked chips using different substrates. For example, data can be stored in memory that is stacked on to the CPU instead of using power-hungry off-chip SerDes or DMA solutions. CDR architectures would continue to evolve in the wireless context.

\subsubsection{Multi-tone signaling in SerDes}

This is a relatively new idea in SerDes design [127] and mimics a communication system on a PCB. A block diagram for a multi-tone SerDes is shown in Figure 2.16.

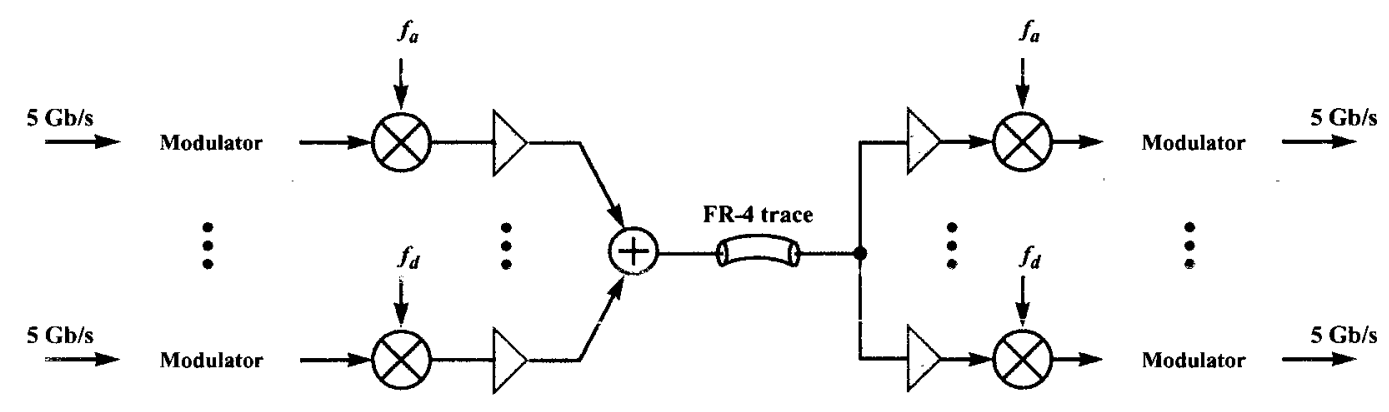

Figure 2.16 Multi-tone SerDes block diagram

In effect, it is similar to using a communication system with 16-QAM encoded symbols ( 4 bits per symbol) using four carriers but the channel has been replaced by the FR-4 PCB trace. At $20 \mathrm{~Gb} / \mathrm{s}$ the attenuation for a 30 " FR-4 trace is $60 \mathrm{~dB}$ with deep notches in the $\mathrm{CX}$ frequency response. This requires multi-tap equalization that consumes considerable amount of power. In short, the FR-4 PCB is not very useful for 20 Gbps NRZ signaling. The multi-tone SerDes architecture multiplexes multiple channels using different carriers pre-emphasized according to the attenuation profile of the trace to deliver an optimum eye at the RX end. This is an analog architecture that requires four locked LCVCOs working at different phase offsets. The reported improvement is in the area of equalization power since it is required over a narrower bandwidth per channel. The purity required for separating different streams is affected by the quality of the quadrature clocks. This architecture would have opened up newer possibilities for increasing the 
serial data throughput between system on a PCB but the shift toward optical communication between systems is, unmistakably, a game-changer. This architecture might still be utilized for inter-module signaling in a SiP scenario.

\subsubsection{Optical SerDes}

Intel $^{(\mathbb{R}}$ corporation has announced Light Peak ${ }^{\mathrm{TM}}$ technology as the optical link that is scalable from $10 \mathrm{Gbps}$ to $100 \mathrm{Gbps}$ for peripheral applications. Another milestone as of Q2-2010 is the announcement of a $4 \times 12.5 \mathrm{Gbps}=50 \mathrm{Gbps}$ link using hybrid Silicon Laser Technology and is a new development in the optical SerDes space [159]. There are two points to be noted; a round figure of $50 \mathrm{Gbps}$ is chosen as it is easier for consumers to remember it and four lanes are being used to deliver this throughput. Transceiver-related developments have also been reported for optical hardware [113][114].

\subsection{Reaching maturity}

For CPUs, tera-flops/W (floating point operations per second) is used as an indicator of throughput per unit power; for SerDes it is Gbps $/ \mathrm{mW}$. Due to Moore's law not being as relevant for CPU bus speeds or raw SerDes bit rates anymore, the CPU vendors have resorted to implementing multiple cores [143] running at lower frequency (and thus temperature) thereby reducing the design complexity and increasing CPU yields. For the case of SerDes, multiple lanes using manageable speeds for the given technology are the key to increasing the throughput whereas digital design enabled by CMOS and the IP reuse facilitated by the digital flow are the keys to a reduced development time. The industry might abandon CMOS, but it cannot abandon digital techniques. The industry cannot reverse the tide on serial vs. parallel links either [84]. This dissertation on all-digital CMOS CDR circuits will, therefore, remain relevant in the current context until a cheaper substrate can be exploited on industrial scale. Optical SerDes may become the dominant interconnection 
mechanism for high-speed peripherals in the 2010-2020 decade following the recent announcement of Light Peak ${ }^{\mathrm{TM}}$.

Few of the major and fundamental problems facing the IC industry are power density, interconnect delays, variability, reliability, yield, modeling and simulation of complex systems. Carbon nanotubes (CNT) may shift some of these fundamental bottlenecks $[144][145][146]$ to different arenas in the coming decades. Parallel processing and gridenabled simulators can alleviate simulation and visualization bottlenecks. In addition 3D SiP technologies, vertical transistors [138], optical device integration [78][158], CMOS nano-MEMS switches and novel nano devices will redefine the SerDes design landscape.

\subsection{Summary}

In this chapter, the SerDes system interconnections were briefiy surveyed in order to appreciate the attendant design challenges. Packaging and SerDes IP usage were also reviewed. SerDes is a "big digital, small analog" problem. It mostly utilizes CMOS technology and-like every other product that does so-benefits from digital IP reuse and scaling. The main components of a SerDes are the DFE and the CDR systems. The DFE opens the vertical eye opening and the CDR cirucit opens the horizontal eye opening to recover the transmitted symbols that have been corrupted by the channel. Other SerDes components enable, assist and complement these functionalities. Equalizer architectures and algorithms are not discussed in this work. This work focuses on CDR systems and an overview of CDR architectures follows in the next chapter. SerDes architectures are showing signs of maturity and have approached physical limits determined by power density [139] and interconnect bandwidth [13]. Testing of SerDes systems is an area unto itself but is omitted here for brevity. There are too many acronyms in the SerDes literature and no dictionaries to explain them. 


\section{Circuits}

"Language is legislation, speech is its code. We do not see the power which is in speech because we forget that all speech is a classification, and that all classification is oppressive."

Roland Barthes

\subsection{Chapter overview}

This chapter presents a survey of CDR architectures from the literature. The pros and cons of the various approaches used over the years are highlighted. The evolving techniques for architectures and components required to realize modern CDR architectures are reviewed. A classical PLL forms the core of a CDR system [22]. The need for a unifying theme in CDR architectures is highlighted.

\subsection{Design of CDR systems}

The questions that drive CDR specifications and design are listed below. These questions provide guidelines for architecture selection and component design.

- Can the phase difference between clock(s) and data at the input be detected accurately at speed? (PD architecture)

- Can the PD data be filtered to reduce unnecessary corrections? (jitter tolerance, bandwidth, filtering and stability)

- How often should the error be compensated without degrading the specified BER? (tracking bandwidth)

- What is the magnitude of the phase correction to maintain the specified BER? (phase step size) 


\subsection{Discrete CDR circuit implementation}

For plesiochronous systems, the clock is not transmitted to save power and has to be extracted from the data stream [92]. A differentiator circuit creates a frequency component at the frequency of the clock by accentuating the rising and falling transitions. This frequency component is rectified using a non-linearity and bandpass filtered (BPF) to provide a clock output. This discrete design requires hand matching of various delays and device parameters and is not suitable for high speed links [32]. A discrete design has been shown to work at 35.1 Gbps [31]. It is impractical to implement the discrete CDR circuit as an integrated design as shown in Figure 3.1, but the numerical cost of the operations involved is negligible for advanced computers and this method can still be used to simulate CDR operation, especially for computation-intensive transient bit error simulations.

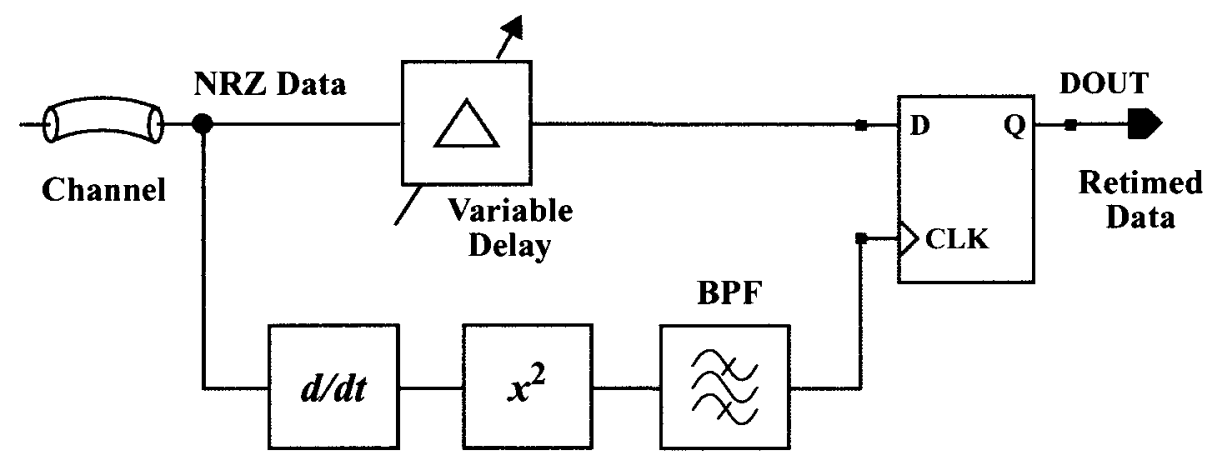

Figure 3.1 Discrete CDR implementation

\subsection{Integrated CDR circuit implementations}

Two choice are available for integrating the discrete CDR circuit. The first is to implement the BPF on chip. The contenders in this regard are SAW filters, MEMS devices and high-Q filters [149]. For mass production and going forward, the high-Q architectures could still be the cheapest and the most reliable technology requiring no extra masks [150]. The price of discrete SAW filters has been steadily reducing and integrated SAW 
devices have been reported [147][148] that can potentially solve a wide variety of oscillator purity problems. The SAW and MEMS devices require extra masks that reduce yield.

Until the above-mentioned techniques can be implemented on a commercial scale, CDR systems are implemented using on-chip PLL blocks to exploit the knowledge accumulated about the PLLs over the decades [135]. A CDR circuit with a PLL mechanism is shown in Figure 3.2 [2] while a more detailed block diagram [22] is shown in Figure 3.3.

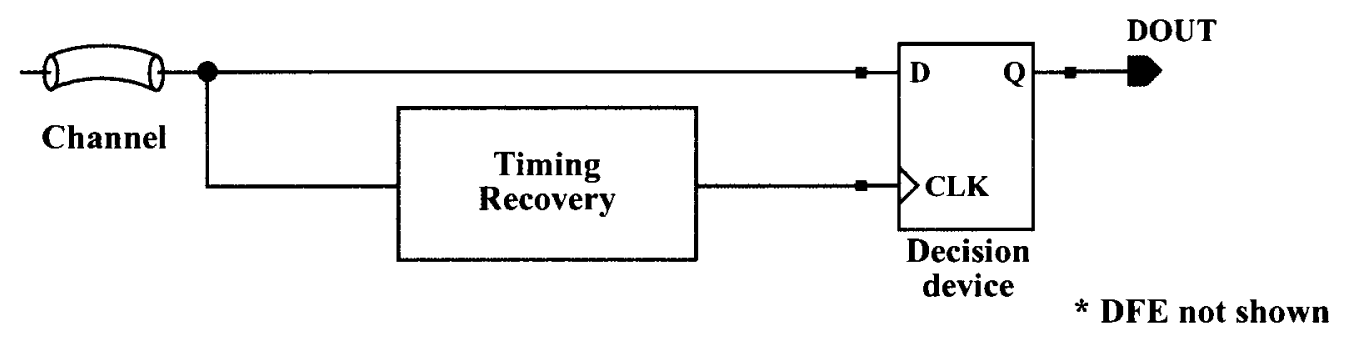

Figure 3.2 CDR concept diagram

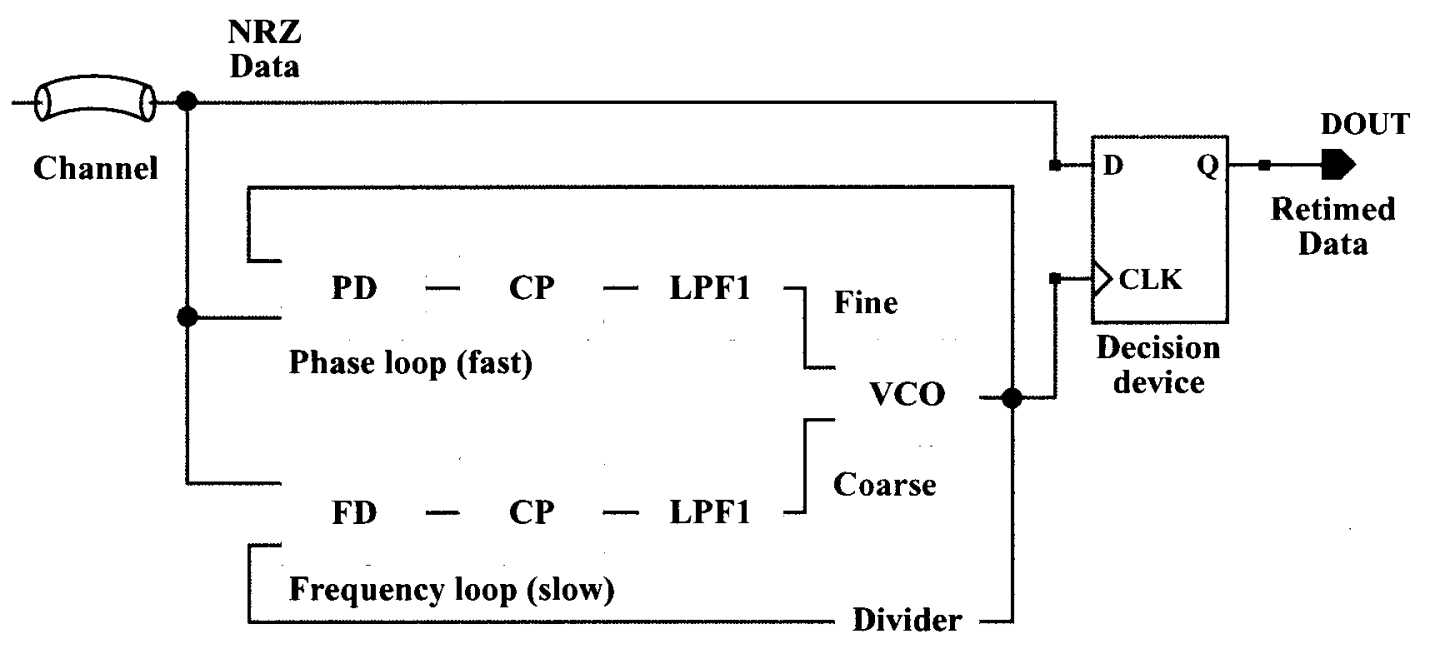

* DFE not shown

Figure 3.3 Conventional CDR block diagram

\subsection{Conventional PLL-based CDR systems}

The conventional PLL-based CDR circuit is referred to as a $2 x$-oversampling $(2 \mathrm{XO}) \mathrm{CDR}$. This is in conflict with the choice of oversampling ratio $(O S R)$ as presented 
later in this work so a pre-emptive clarification is in order. A feedback loop exists in the conventional PLL-based CDR circuit that acquires the frequency and aligns one of the edges of the data and the clock so that the other edge of the clock (assuming a $50 \%$ duty cycle) can be used to sample the data at the centre of the UI. This full-rate linear CDR operation is shown in Figure 3.4. It is obvious that sampling the data stream 'twice' in the period means 'once only at $0.5 \mathrm{UI}$ away from the tracked phase' or else the CDR design objective is not met. The conventional CDR system is, strictly speaking, not a $2 X O \mathrm{CDR}$ but a linear CDR mechanism that uses two conveniently available edges with infinite granularity once the lock has been achieved. The presence of asymmetry in RJ and DJ or duty cycle distortion (DCD) can seriously degrade the performance of this type of CDR system.

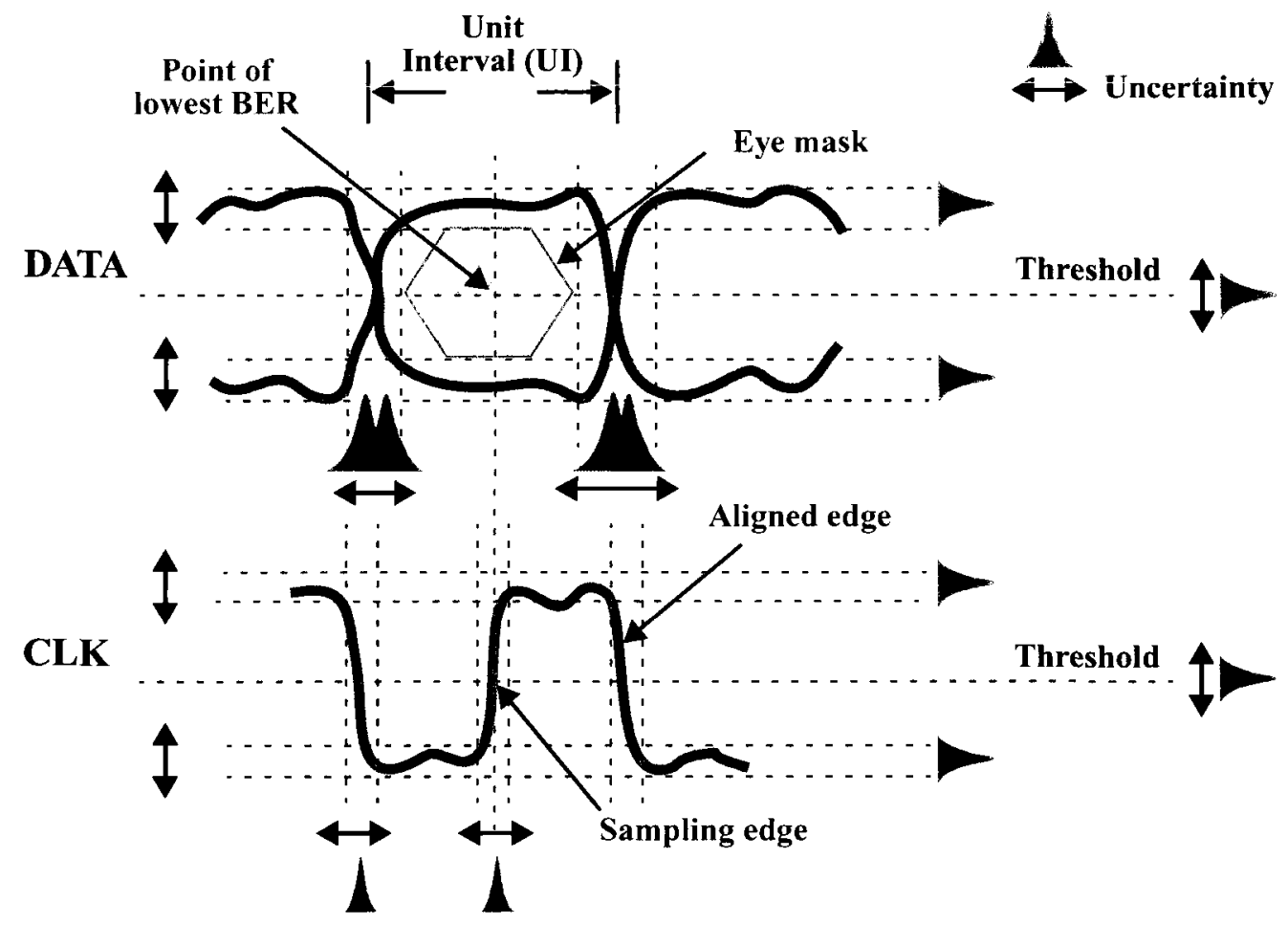

Figure 3.4 Challenges in designing CDR circuits

Once the clock edge is recovered, data recovery can be performed by sampling the data stream with this recovered clock using a decision device that is a flip-flop as shown in Figure 3.2. The architecture shown in Figure 3.3 is the basis of analog PLL-based CDR 
circuits [22]. The advantage of using a PLL for timing recovery is that it can be integrated. The frequency acquisition is not robust and a much slower frequency loop is utilized to maintain center frequency [105]. The interaction between the phase-tracking loop and the frequency-acquisition loop should be minimized to achieve better stability.

The understanding of the term 'analog' generally implies that the PLL loop filter uses passive components and that the Voltage-controlled oscillator (VCO) is also continuously controlled. A PLL employing a PFD, CP, VCO and a digital divider is known as a digital PLL; the nomenclature is erroneous but is traditionally used. The CDR circuits are broadly classified as linear or bang-bang (or digital) according to the type of the phase detector. The key points for conventional analog CDR circuits are as follows:

- Only a single edge of clock and data is aligned and tracked.

- The other clock edge is assumed to be $0.5 \mathrm{UI}$ apart.

- The common mode thresholds have to be maintained at the correct level.

- Supply- and substrate-noise contributions reduce the vertical eye opening.

- Jitter reduces the horizontal eye opening.

- Asymmetric jitter and DCD degrade the BER for conventional CDR systems. The timing margin of analog CDR systems is therefore limited to a maximum of 0.5 UI-pp.

\subsection{CDR Components as a Method of Classification}

It is not rigorous to classify a CDR circuit based on its components but like many things in engineering, it is often attempted with impunity. The partitions along the analog/ digital line based on component choices are arbitrary, ineffective and incoherent. Components are used for their competitive advantage and cannot be used as pivots during a classification exercise. This section reviews the components widely used in CDR architectures from the all-digital perspective. It is assumed that the reader is familiar with PLL systems 
[2]. Once the components are discussed, their use will be examined at a higher level in CDR systems that have used them over the years.

\subsection{Phase Detectors}

The phase detectors for CDR circuits need to run at the data rate in order to provide meaningful information. This is in contrast to the PLL-FS where a feedback divider relaxes such a constraint. A PD forms the front-end of the CDR system. If the measured phase difference is inaccurate, then the subsequent tracking decisions can only be similarly erroneous. The speed and resolution of PD circuits is, therefore, crucial to the operation of CDR circuits. A review of PDs is available in the literature [30].

\subsubsection{XOR phase detector}

An XOR gate is an odd parity detector. Consequently, a 2-input XOR detects the difference in logic levels for binary input signals making it suitable for measuring the phase difference as shown in Figure 3.5.

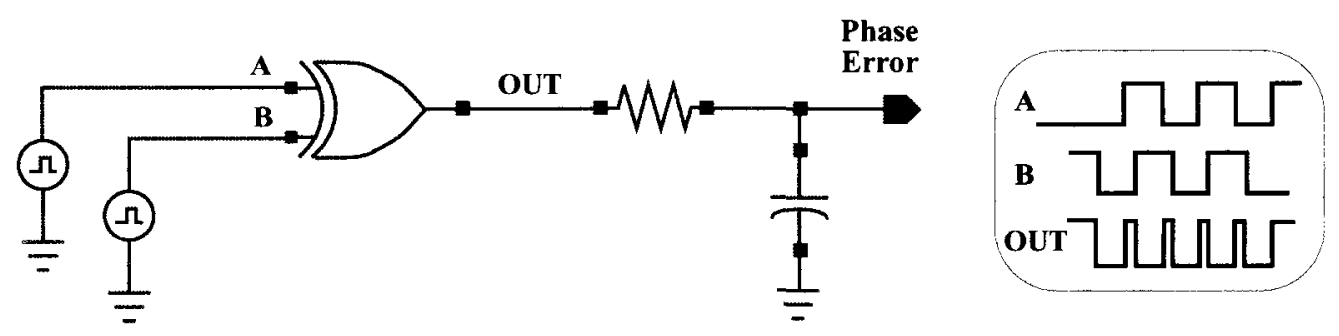

Figure 3.5 XOR PD

The LPF at the output of the XOR can be implemented as a shift register, counter or a passive RC-circuit. An XOR PD is a memory-less device and cannot measure the frequency difference. Moreover, different inputs can cause similar average outputs resulting in false locking. Another complication is that the PRBS sequence has missing transitions so the applicability of XOR PDs to the CDR domain is reduced. 


\subsubsection{Tri-State phase frequency detector}

The tri-state Phase Frequency Detector [24] is widely used in PLL-FS architectures as it provides both frequency and phase detection. It can provide UP and DN signals in response to a phase and frequency difference. For data recovery, however, the absence of data edges would confuse the PFD into making a wrong decision about the instantaneous frequency of the incoming data. It is used during CDR acquisition phase as part of a dual loop arrangement and is subsequently disabled as a more linear Hogge's style PD takes over [22]. A four-state PFD that ignores the missing data transitions using an additional delay line has been presented in the literature [28][29].

\subsubsection{Hogge's phase detector}

The Hogge's PD (HPD) [25] provides proportional and reference puises for detecting the phase difference when data transitions are absent [22]. A frequency detection (FD) circuit is required to bring the circuit within locking range [92]. The block diagram of the HPD is shown in Figure 3.6.

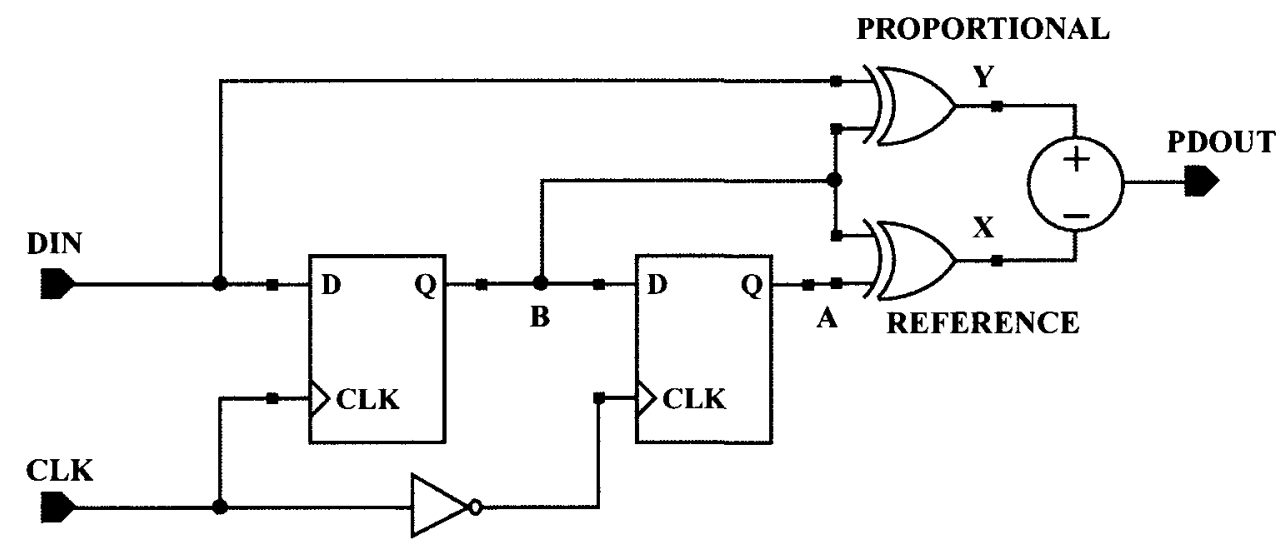

Figure 3.6 Hogge's PD block diagram

It is supposedly a linear system and the primary difficulties arise as a result of $C L K-Q$ and $D-Q$ delays in the flip-flops resulting in a systematic skew in the sampling point that reduces the JTOL and degrades the BER. The CP mismatch due to leakage, lay- 
out and PVT variations results in additional skew. As the voltages shrink, the process variability rises and VCO gain increases in VDSM technologies, the ripple on the $V_{\text {cont }}$ signal shifts the sampling point too abruptly to maintain the desired BER. If CDR bandwidth restrictions prohibit filtering of this ripple, then a solution could be to use fast- and slowpaths in the analog VCO to provide reduced sensitivity to the ripple as shown in Figure 3.3. The frequency acquisition of a HPD is not robust and a cycle-slip detector along with a frequency detector needs to be present [92][93]. Even during a locked scenario, the ripple causes activity on the $\mathrm{CP}$ due to the half-cycle skew between the proportional $(\mathrm{Y})$ and the reference $(\mathrm{X})$ pulses [22]. An open-loop simulation depicting the effect of ripple on the $V_{\text {cont }}$ signal is shown in Figure 3.7 for a $1.0 \mathrm{GHz}$ PRBS9 signal.

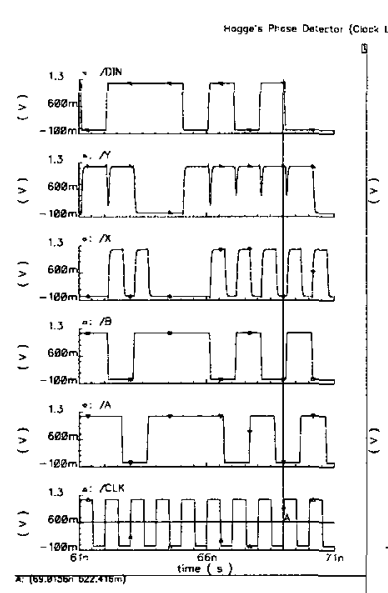

(a) UP

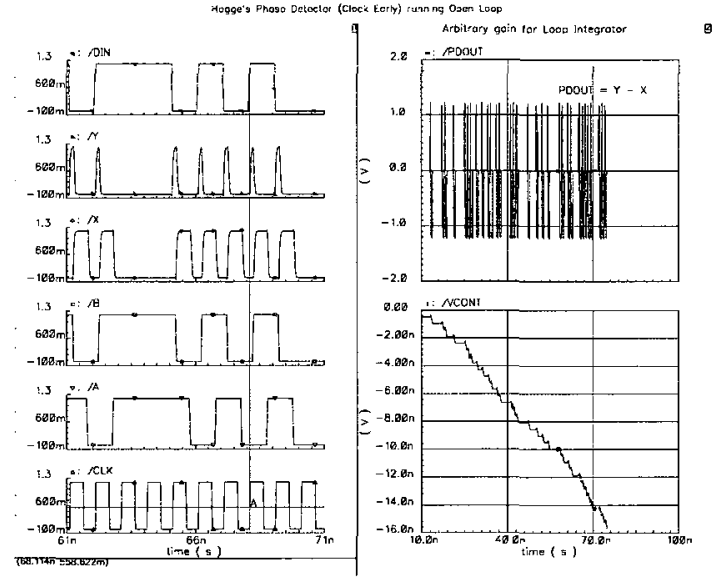

(b) DN

Figure 3.7 Hogge's PD operation (open loop)

The frequency acquisition loop based on a tri-state PFD is enabled when a cycle slip is detected and is disabled when the lock detector (LD) circuit signals the end of acquisition. This closed loop operation of the HPD is demonstrated in Figure 3.8.

As far as the simulation is concerned, no useful performance or test data can be acquired until a lock is achieved and that consumes a long time in an AMS simulation of a 
CDR circuit with widely differing time constants. In case of loss of lock, the acquisition procedure is re-initiated consuming more time.

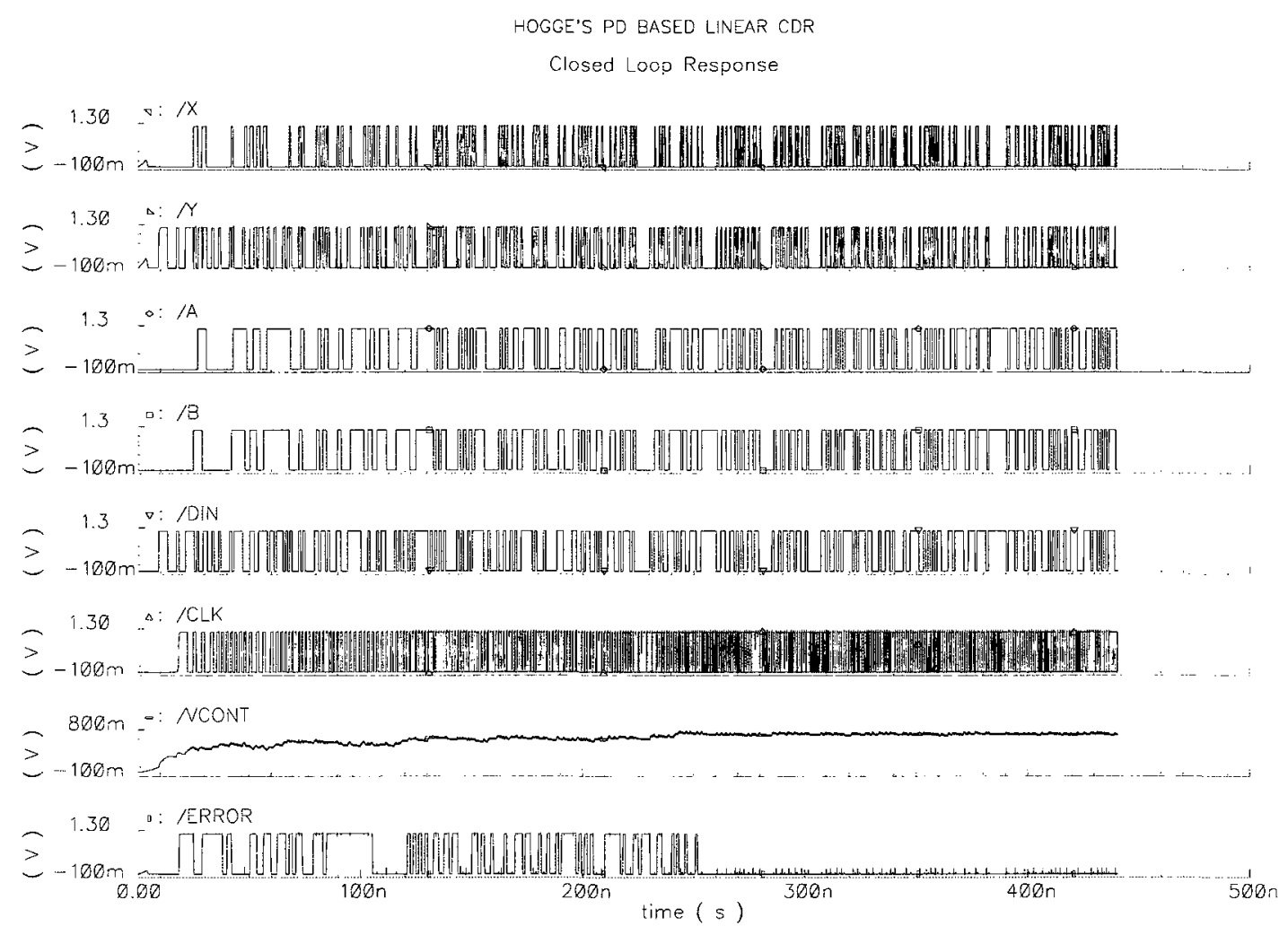

Figure 3.8 Hogge's PD style linear CDR response

\subsubsection{Tri-wave Phase Detector}

The Tri-wave PD [128], shown in Figure 3.9, offers improvements over the HPD in terms of the instantaneous deviation of the sampling point. This occurs since the average area under the triwave output is cancelled for zero phase difference. In contrast the sampling point for a classical HPD in the locked condition is systematically skewed and 
this degrades the BER performance of the HPD-based CDR circuit. A transient simulation using the tri-wave PD is shown in Figure 3.10.

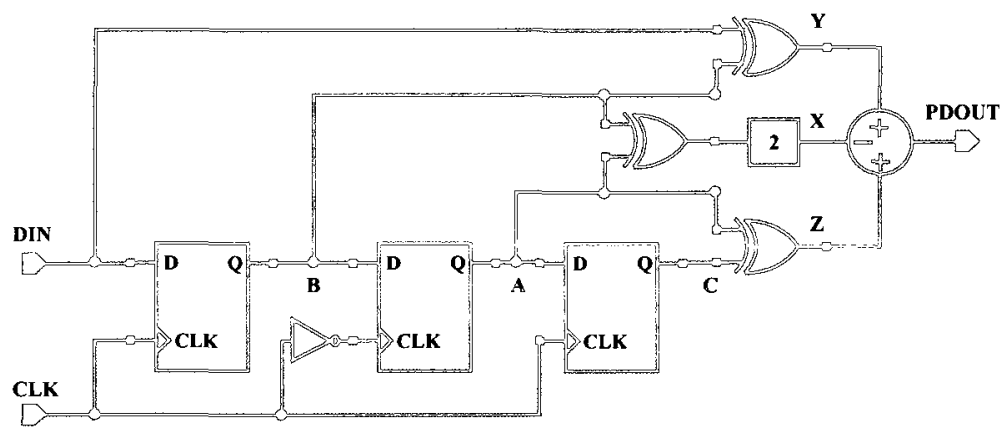

Figure 3.9 Triwave PD block diagram

TRI-WAVE PD BASED LINEAR CDR

Closed Loop Response

- $1.30 \therefore$ /ERROR

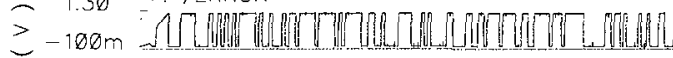

$-1.3 \times:$ DIN

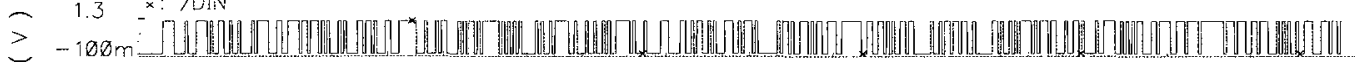

$-1.3 \quad \mathrm{a}: \mathrm{B}$

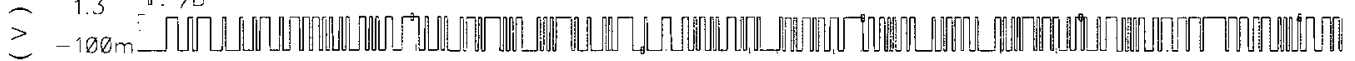

$-1.30 \therefore:$ CLK

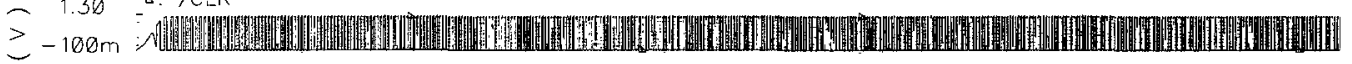

$-1.30 \therefore: / 2$

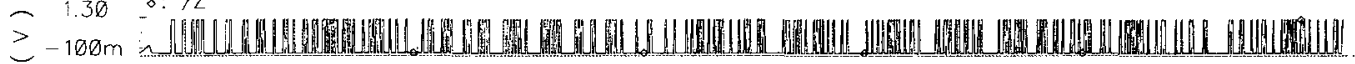

$-1.30-\therefore / x$

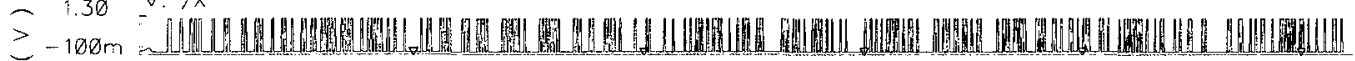

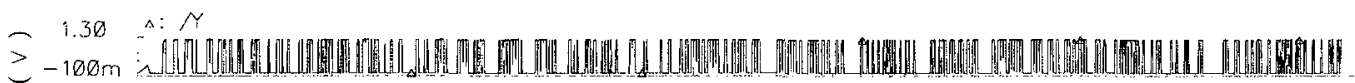

$-1.3 \therefore: 10$

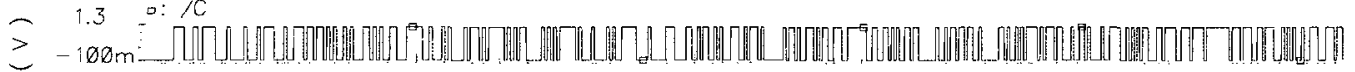

$-1.3 \div$ : $>$ A

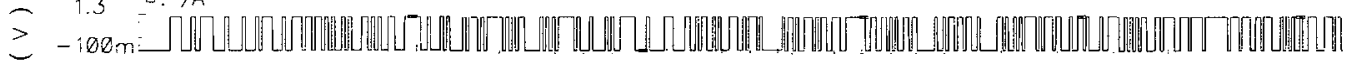

- $800 \mathrm{~m}-\therefore$ NCONT

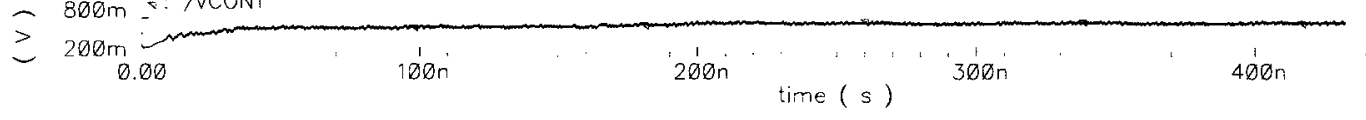

Figure 3.10 Triwave PD style linear CDR response

The displacement of the sampling point in the Triwave PD about a mean position degrades the JTOL but not as drastically. In addition, it alleviates the problem of a system- 
atic skew in the sampling point that is present with the classical HPD design. The triwave PD suffers from increased sensitivity to the clock duty cycle and a modified tri-wave PD has been proposed to mitigate the same [128]. The power consumption increases with the triwave and the modified triwave PDs as the additional flip-flops and XOR gates operate at the incoming data rate.

\subsubsection{Linear PD limitations}

Historically, as data rates increased, the ability of the processes to provide a working flip-flop at full data rate got challenged every two years. The PVT-related delay variations through the flip-flops and the XOR gates translated into increased total jitter at the output resulting in a degraded BER. The CDR circuit was a product of predominantly ana$\log$ thinking, so a half-rate linear PD was theorized to address this design challenge.

\subsubsection{Half-rate linear PD}

There are three challenges that need to be resolved when using full rate PDs. The first issue is that the oscillator running at high speed often provides reduced spectral purity and tuning range. The second issue is the speed requirement imposed upon the flipflops. The third issue for a HPD is the systematic skew for zero phase difference as proportional pulses are wider by the $C L K-Q$ delay of the flipflop. At high bit rates, the ripple owing to the half cycle delay between the proportional and reference pulses is also untenable as the ripple on a traditional oscillator can unlock the CDR circuit. A reduction of $K_{v c o}$ can alleviate the ripple by reducing the CP current but CDR bandwidth is reduced as well.

The idea behind a CDR circuit utilizing a half-rate PD is that both edges are utilized for phase detection, thus alleviating the speed requirement for latches and the oscillator by half. A half-rate linear PD has been presented in the literature [22] and is shown in Figure 3.11. The level sensitivity of the latches is depicted using a symbol at the centre of the respective latches. Reference and proportional pulses are generated using four latches 
that provide complete information about the phase difference between clock and data. The demultiplexed data is present at the DOUTA and DOUTB outputs.

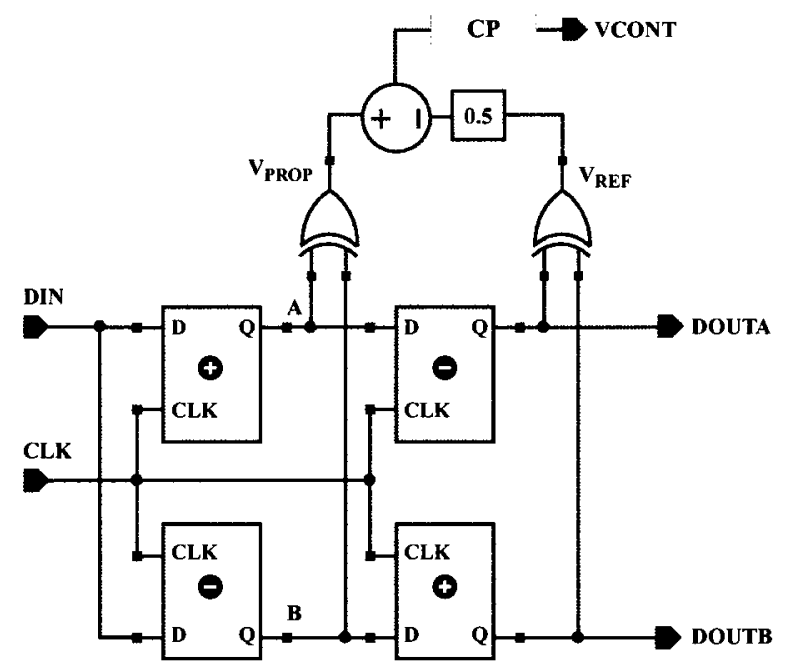

Figure 3.11 Half-rate linear PD block diagram

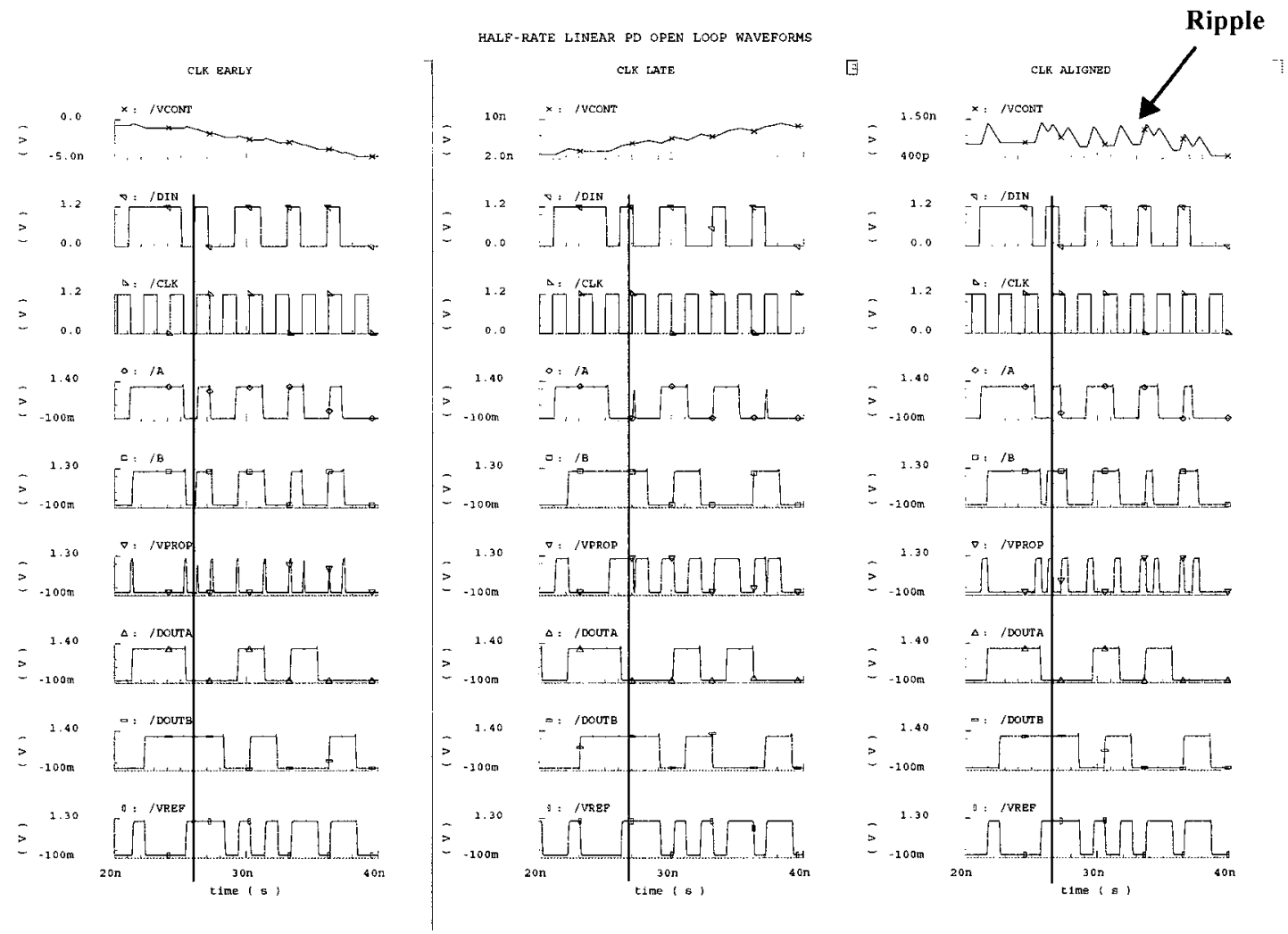

Figure 3.12 Half-rate linear PD block diagram and operation 
A set of publications discussing the half-rate linear and binary CMOS CDR implementations have reported [99][100] that using a $0.18-\mu \mathrm{m}$ process, a full-rate circuit would have been restricted to $7 \mathrm{GHz}$ but a half-rate $\mathrm{CDR}$ architecture was able to deliver $10 \mathrm{Gbps}$ throughput using both edges of a $5 \mathrm{GHz}$ oscillator thereby circumventing a technology limitation.

\subsubsection{Reduced-rate linear phase detectors}

Extending the theme of the half-rate PDs, reduced-rate PD-based CDR architectures have been reported in the literature. These include the quarter-rate [101], the $1 / 8$ thrate [102][104]. Generalized analysis of the reduced rate architectures [103] suggests that this theme cannot be continued indefinitely due to area and power requirements. Also, the inter-phase spacing variance puts a limitation on the achievable BER. Energy-wise there is no major advantage in using a reduced-rate PD architecture since the number of transitions for CDR operation remain the same. Only the portion of power dissipated in the parasitics would be reduced due to lower VCO frequencies. The reader can notice in Figure 3.12 that the ripple is still present in the locked state and the original problem in the HPD design has not been resolved but rather postponed pending a new discovery.

\subsection{Transitioning from linear to digital phase detectors}

There is nothing elegant, linear or mathematically superior about the linear PD. Like everything else, it is an accident of history and circumstance. The simple argument that a linear PD generates pulses whose difference is proportional to the phase difference has too many invisibles that mask the physical engineering reality. First, it is assumed that the technology's $f_{\tau}$ allows the narrow current pulses at the CP output. The narrow pulses are unavoidable since the PD would be hovering around the optimum sampling point during locked operation. Such narrow pulses are filtered due to their high frequency content resulting in unpredictable blips of current in the analog CP. It is further assumed, even if 
the short pulses were not the bottleneck, that the difference circuit would generate the ideal difference. This implies perfect switches with no charge injection. The assumption that there is no intrinsic delay through the PD circuitry is not obvious. A PD is a device trying to quantify the delay between two edges in real time. The intrinsic PD delay is uncorrelated with the input data and contributes to the output jitter.

It is evident that the assumption of linear phase difference breaks down for physical reasons_-but not mathematical reasons - as the locked state is approached. For 'zero' phase difference, the activity on a linear PD still produces corrections-half a cycle later-and serve to dither the sampling point for several reasons such as jitter, skews, delays, temperature, process, leakage, quantum effects, supply disturbances, substrate injection and a host of unmodeled and yet unforseen factors as geometries shrink further.

In the limit, it follows that the decision circuit never encounters a 'zero' phase difference state for long due to thermal jitter, if nothing else. The problem, therefore, reduces to whether it is necessary to have a linear PD at all since a recovery circuit would spend a clear majority of its running time in the locked state and the very small phase differences can be encoded as whether the incoming data edges occur earlier or later than the ideal sampling point (or vice versa) without regard to the absolute deviation from that reference point. Another valid question is whether if two consecutive data edges happen to be early and late due to random effects, does it even make sense to change the sampling point?

On most occasions, the phase deviation is small and swaying on both sides of the sampling point, and therefore it is to be labeled as high-frequency jitter and ignored. If the deviation starts accumulating in a certain direction, the corrective action needs to be taken with enough agility to recover the data. The ability to detect the direction of the data edge movement, i.e., jitter, from cycle to cycle or every so many cycles (once lock is achieved) is more important than finding the absolute deviation at any given instance. The stray 
edges that are further away from the locking point are to be suppressed. The edges close by that are oscillating from early to late status are to be filtered.

\subsubsection{Alexander PD or Bang-Bang PD or Early-Late PD}

The PD based on the insight that an early or late indication is sufficient is variously known as the Alexander PD [26], Bang-Bang PD (BBPD) or an Early-Late PD and is shown in Figure 3.13 along with a summary of its operation.
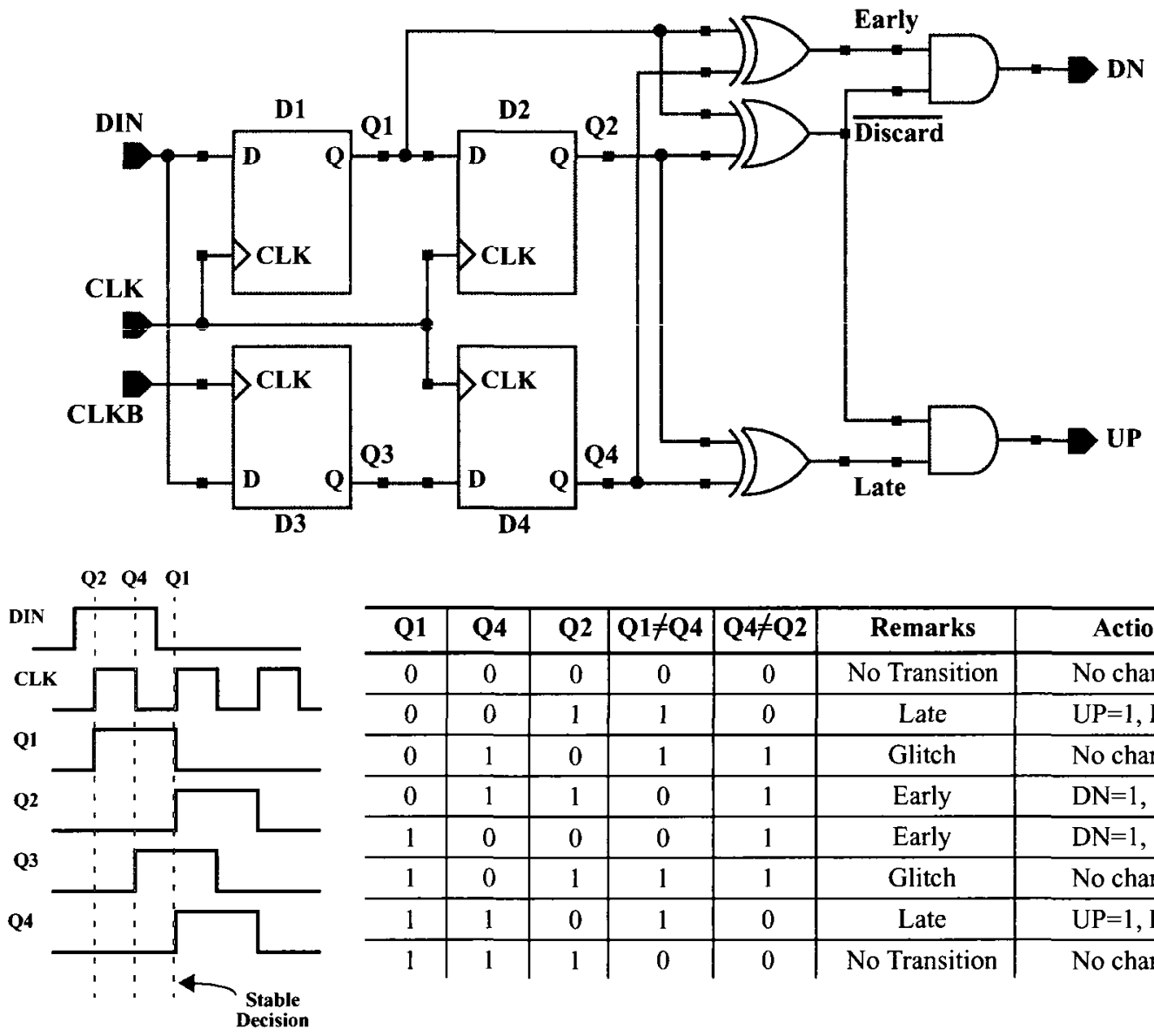

\begin{tabular}{|c|c|c|c|c|c|c|}
\hline Q1 & Q4 & $\mathbf{Q 2}$ & $Q 1 \neq Q 4$ & $Q 4 \neq Q 2$ & Remarks & Action \\
\hline 0 & 0 & 0 & 0 & 0 & No Transition & No change \\
\hline 0 & 0 & 1 & 1 & 0 & Late & $\mathrm{UP}=1, \mathrm{DN}=0$ \\
\hline 0 & 1 & 0 & 1 & 1 & Glitch & No change \\
\hline 0 & 1 & 1 & 0 & 1 & Early & $\mathrm{DN}=1, \mathrm{UP}=0$ \\
\hline 1 & 0 & 0 & 0 & 1 & Early & $\mathrm{DN}=1, \mathrm{UP}=0$ \\
\hline 1 & 0 & 1 & 1 & 1 & Glitch & No change \\
\hline 1 & 1 & 0 & 1 & 0 & Late & $\mathrm{UP}=1, \mathrm{DN}=0$ \\
\hline 1 & 1 & 1 & 0 & 0 & No Transition & No change \\
\hline
\end{tabular}

Figure 3.13 Alexander PD block diagram and operation

The Alexander PD operates as follows. Flipflop D1 samples the data and delays it by a clock cycle using flipflop D2. Flipflop D3 samples the data at the negative clock edge and delays it by half a clock cycle. Hence Q1 and Q2 are the midbit symbols, where as Q3 is the symbol that should ideally align with the other clock edge. All of these transitions 
are compared at the same time to provide the correct phase difference information. If Q1 and Q2 are the same as Q4 (i.e. the transition sample Q3 now delayed by half a clock cycle to give Q4) then there has been no transition and no decision can be made. If the clock arrives earlier than the ideal sampling point (as shown in Figure 3.13), the last two samples $(\mathrm{Q} 1, \mathrm{Q} 4)$ would be different than the first two $(\mathrm{Q} 2, \mathrm{Q} 4)$. The last two samples $(\mathrm{Q} 1, \mathrm{Q} 4)$ would be the same if the clock arrives late and the first two samples (Q2,Q4) would be different. If the consecutive midbit samples (Q1,Q2) are the same but the transition sample Q4 is different, then it would signify a glitch that is to be discarded. The high gain in the vicinity of the sampling point is mitigated by flipflop metastability [33]. In the absence of

a data transition, there is no activity on the $V_{\text {cont }}$ line; the VCO drift occurs only due to $\mathrm{CP}$ leakage and noise. This is an advantage over analog PDs where a ripple exists for zero phase difference due to charge summation operations. The BBPD architecture is realizable as long as flipflops can be designed for a particular process node [32]. For digital CDR circuits, the UP and DN signals are filtered and subsequently drive the oscillator. The difficult design of a low-voltage CP in a VDSM technology is obviated.

\subsubsection{Half-rate BBPD}

As the early/late or digital mentality took hold in CDR design, an obvious extension of the idea emerged. The half-rate schemes could theoretically provide the same benefits for digital PDs as they had delivered for linear PDs [100]. Hence an important theme in CDR design is the use of multi-phase clock generators to alleviate speed requirements for flipflops. A half-rate BBPD along with the operational waveforms is shown in Figure 3.14 [22]. The samples Q1, Q2 and Q3 serve the same purpose as in a full-rate BBPD; but for a half-rate system, a quadrature clock (CLKQ) is used to sample the data as 
well to provide adequate phase resolution. The half-rate PD eases the speed requirements for flipflops.
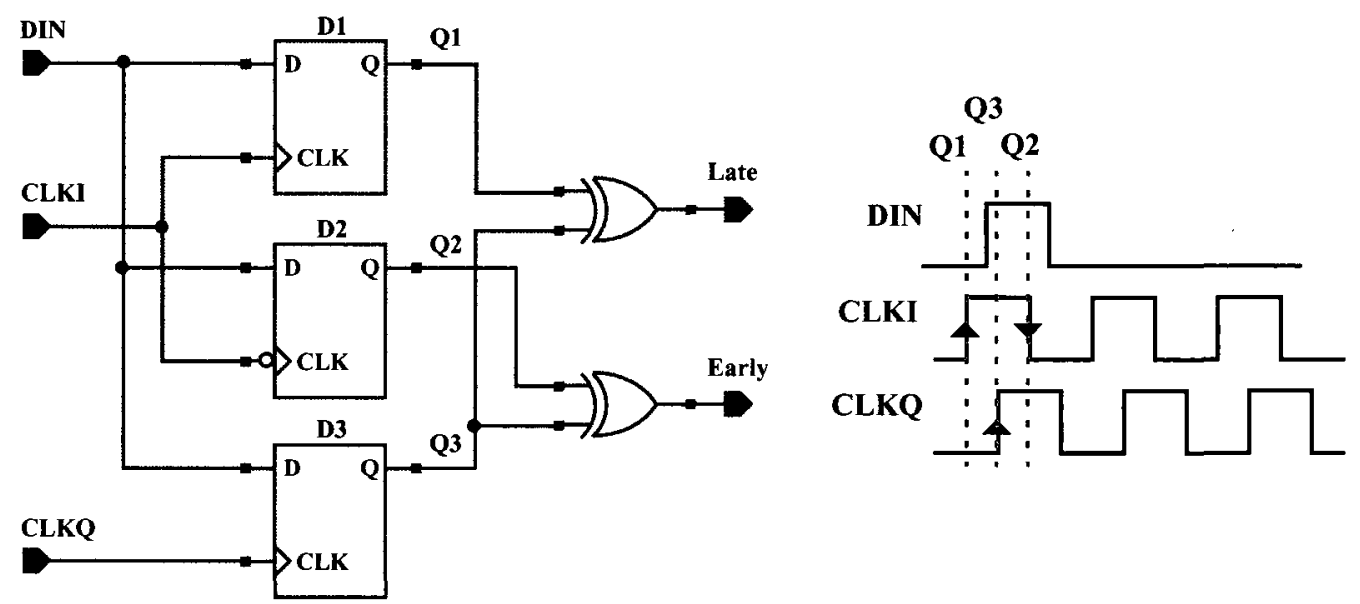

Figure 3.14 Half-rate Binary PD and operation

\subsection{Multi-phase and multi-bit phase detectors}

Sampling the data at multiple points with the available clock phases can provide digital information on the phase difference with improved granularity. This is termed oversampling. Henceforth, a $2 x$-oversampling PD is denoted as a $2 \mathrm{XO} \mathrm{PD}$. The abbreviations can be extended to $3 \mathrm{XO}, 4 \mathrm{XO}, \ldots, \mathrm{NXO} \mathrm{PD}$ as the oversampling ratio $(O S R)$ changes. An illustration of phase detection using multiple phases is depicted in Figure 3.15.
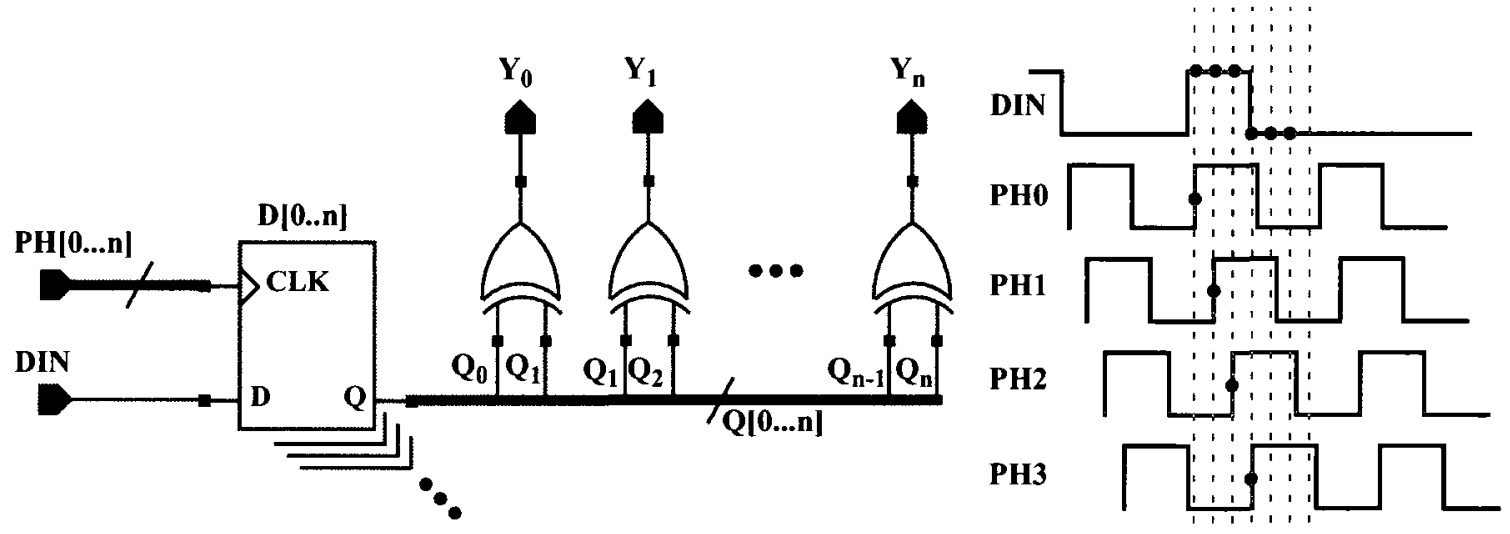

Figure 3.15 Multi-phase detectors and operation 
Oversampling provides additional information. With more phases available, a phase selection can be made in various ways to stay at the centre of the UI when the jitter moves in a certain direction. As a minor downside, the multi-phase oscillators are technologically challenging to implement at very high frequencies. The NXO PD circuits have a better acquisition performance and less output jitter [39]. Oversampling PDs are discussed in Chapter 4.

The realization that the jitter sways on either side of the sampling point and corrections do not need to be made every cycle further weaken the idea of a conventional CDR mechanism that tunes continuously. Given this insight, a data stream can be sampled over several bits at once resulting in the possibility of reduced-rate oversampling CDR architectures. A scaling factor can be applied to the outputs resulting in graded PD output characteristics that more closely resemble a linear loop without the attendant design problems and power dissipation.

The discussion on PDs has elaborated upon the point that the consistency of interphase spacing between adjacent phases available from a multi-phase oscillator has become the challenge and not the frequency of the oscillator itself. High throughput systems can be realized using slower multi-phase oscillators.

Oscillators can exist inside wideband PLL systems that gives these systems PVT invariance for output frequency (and consequently for inter-phase spacing) over the tuning range with poor quality IC compatible passive elements [37]. Another popular choice for multi-phase clock generation is the Delay-Locked Loop (DLL) type system. For DLL systems, the excess phase does not accumulate in a feedback loop and a DLL system is considered more stable than a PLL system. 


\subsection{Frequency Detectors}

At start-up, the local oscillator in the PLL starts from a frequency that is far removed from its final value. Consequently, a mechanism is required to pull the local oscillator close to the final frequency such that the PD with its limited pull-in range can take over. The NRZ data received from the DFE is assumed to be the output of a compliant transmitter that has acquired jitter over the unknown channel. Many FD architectures have been demonstrated over the decades [55]. These are based on quadri-correlators, rotational frequency detectors and the detection of consecutive multiple edges on the same input. Their digital variants work at lower frequencies but enhance integration and portability. The incoming data is sampled with the CLKQ and the CLK is sampled with the input data (DIN). This creates a beat note that is low-pass filtered to get a DC component that drives the oscillator to within the pull-in range of the PD. One such implementation [92][93] has been shown in Figure 3.16.

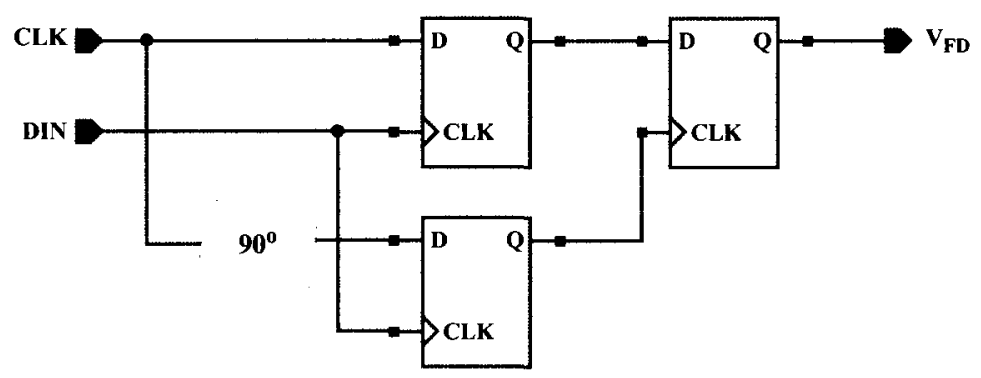

Figure 3.16 Rotational frequency detectors

\subsection{Integrated LC Oscillators}

Oscillation provides the beat in synchronous electronic circuits. The VCOs have held sway over the IC design world since the days of electronic tubes. It has taken a surprisingly long time after the maturity of digital techniques for the digital control mechanisms to be introduced to commercial chips resulting in digitally controlled oscillator (DCO) architectures [12]. 
As soon as the oscillator design problem is phrased in a manner that requires a 'voltage' to 'control' the oscillator, the same needs to be generated. Since this is a continuous signal, the analog mindset takes over the system design of the PLL or the CDR circuit and leaves an indelible impression on the output in the form of jitter (phase noise and spurs). There is no shortage of discussions about oscillators in the open literature.

A fundamental paradigm shift has occurred in oscillator control [38] that is steadily garnering wider acceptance. An all-digital CDR architecture employing a DCO can be seen as the convergence of many techniques. To reiterate, a DCO is not a digital oscillator, it is a digitally-controlled oscillator. The stages of this architectural evolution are shown in Figure 3.17.

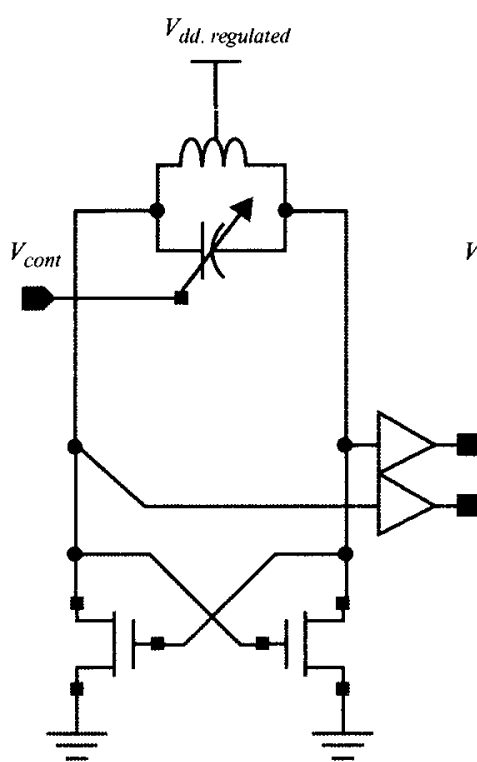

VCO with a coarse varactor $L C$-tank

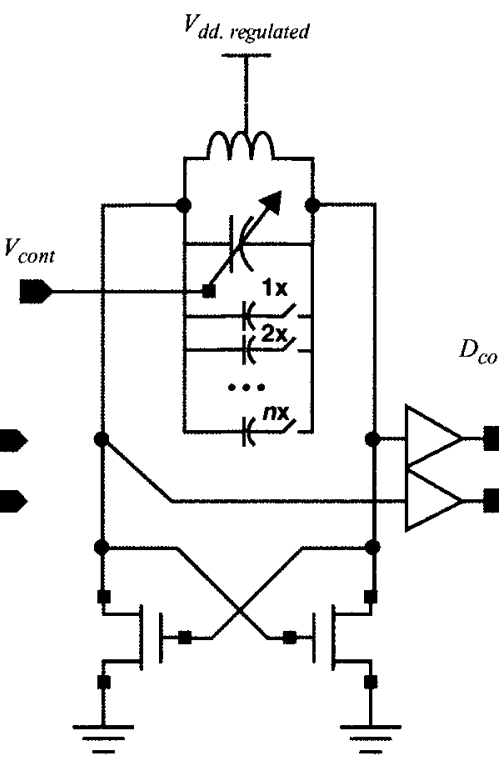

VCO with a fine varactor $L C$ tank plus binary-weighted band-select capacitors - Note the evolving digital control switches - still a VCO

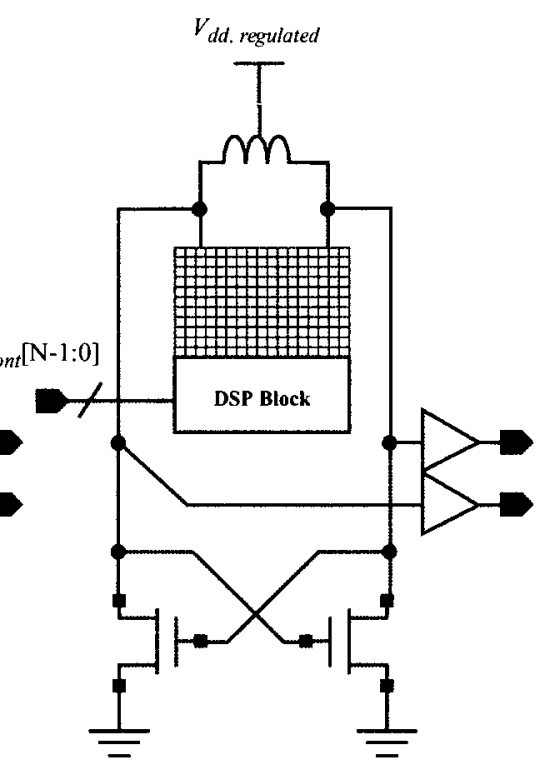

DCO with an array of similarly sized or unit 'varactors' that are randomly turned on or off to minimize spurs and layout-related effects

Figure 3.17 From VCO to DCO - The paradigm shift

If the varactors in the $L C$-tank can be laid out with sufficiently small dimensions and be controlled individually using digital signals, then the oscillator frequency can be 
changed without requiring an analog voltage that acts on the resonant tank en masse causing a spurious havoc. A register can hold the information on how many and which varactors should be turned on in the $L C$-tank at any given instant. If this granularity is not enough, then a modified scheme is used where a subset of varactors is controlled by a sigma-delta modulator [7][8] to achieve finer resolution. Before a DCO is used in a CDR system, a discussion of the digital control approach is presented. Few things as traditional as the VCO change easily; there must be practical reasons to even consider it.

\subsubsection{Transformation to a DCO}

There are four major external gateways that can let noise into an oscillator; the supply, the ground, VCO control input and the EMI interference. Then there is the internal noise from the VCO itself. The noise performance of an oscillator depends on circuit strategies that manage or block these external gateways. These are depicted in Figure 3.18.

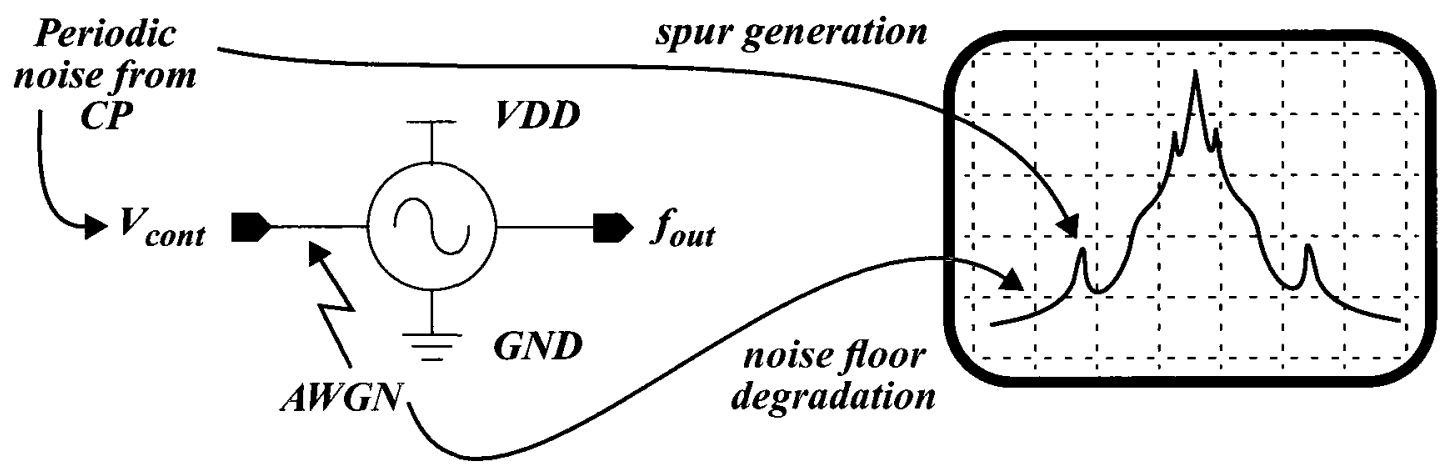

Figure 3.18 Noise gateways into the oscillator

The relationship between the supply disturbance and VCO output depends on the architecture of the VCO. The accepted remedy in commercial chips is the use of a voltage regulator running at a higher input voltage and utilizing thick-oxide transistors. It provide a quiet and regulated voltage to the oscillator that is itself operating at much higher frequencies and cannot use the high-threshold, thick-oxide devices. The injection from the substrate can be minimized by using 'moats' or deep wells that isolate the VCO from sur- 
rounding circuitry owing to the reverse bias arrangements. The inclusion of a capable regulator is a rule and not an exception in commercial SoC systems.

The problem remains the direct connection of an analog voltage to the varactors in the $L C$-tank. Due to current mismatches in the CP, noise on the interconnects, or layout issues, the perturbations at the $V_{\text {cont }}$ input are reflected as spurs or the elevation of the noise floor at the VCO output. This is determined by the VCO gain $\left(K_{v c o}\right)$. Either the CP design must be modified to use less current or $K_{v c o}$ has to be reduced-and both strategies reduce the loop bandwidth. Optimally, the CP is modified to supply larger currents during acquisition but smaller currents are used during normal operation. The band-select capacitors in the $L C$-tank allow the partitioning of the problem on the VCO side. A coarse and fine tuning strategy is thus born. These design strategies mitigate the problem, but do not solve it. A noise on the $V_{\text {cont }}$ input still modulates the VCO output; albeit gently. An interim strategy can only result in short-term relief.

With advanced VDSM technologies, the leakage in the CP circuit and variations in the CP current sources over PVT corners erode the low CP current advantage. The reduced supply voltage increases the $K_{v c o}$ - while the AMS design complexity keeps increasing due to process node migration. For example, a $10 \mathrm{Gbps}$ system experiencing a repetitive $10 \mu \mathrm{V}$ square wave at the $V_{\text {cont }}$ input with a $K_{v c o}$ of $1 \mathrm{GHz} / \mathrm{V}(10 \%$ of tuning range) would experience close to a $10 \mathrm{kHz}$ instantaneous frequency offset. This reduces the JTOL budget of a SerDes system and degrades the BER.

The current in the CP cannot be reduced to zero in the limit as this would open the loop and render the very strategy futile. The binary-weighted capacitors can still be used in the VCO, but the oscillator remains sensitive to the continuous analog voltage. If an array of unit-sized varactors, each controlled with a digital signal, is put in the tank then any continuous voltage applied to the $L C$-tank is removed. A VCO is then transformed into a DCO. As an added benefit, the dithering engine can be synthesized automatically 
and re-used later when a node migration occurs. Such a DCO is robust against varactor layout inconsistencies, cross-talk and spurious modulation and has improved PVT compliance [38].

This preceding discussion is based on the novel and elegant design solution implemented by Texas Instruments for commercial GSM radio chips. In order to further explore it, the CMOS varactor characteristics have to be briefly reviewed.

\subsubsection{Varactors in CMOS processes}

A MOS varactor has different regions of operation as shown in Figure 3.19.

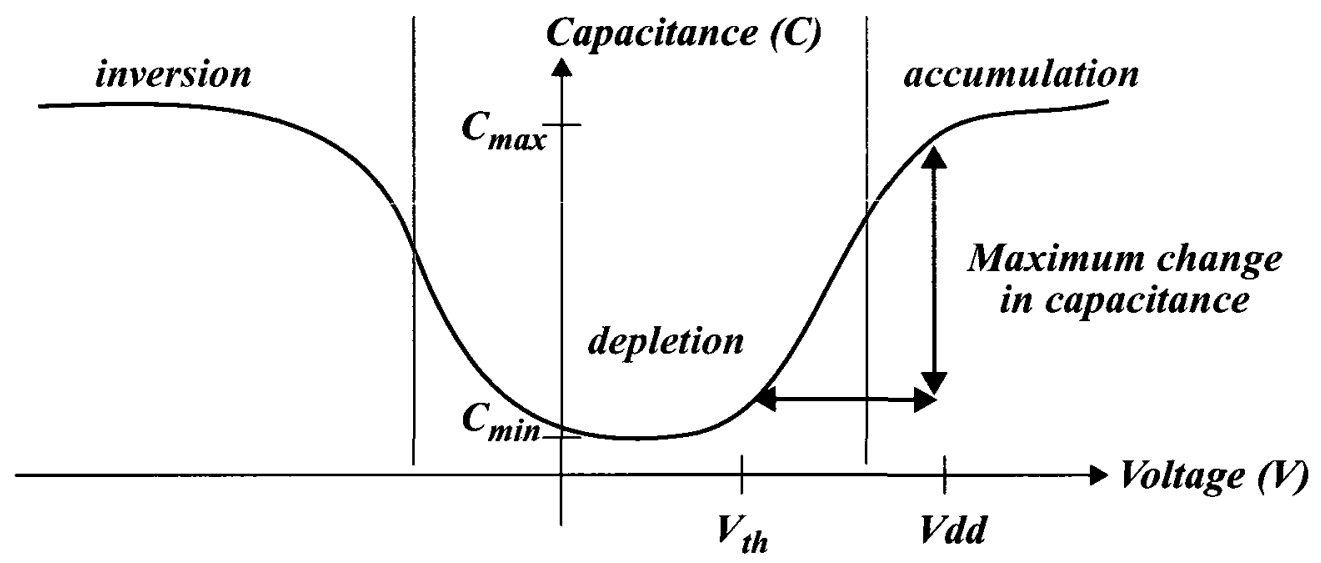

Figure 3.19 MOS varactor characteristics

The unit varactor size is limited by the design rules for a certain process node. A careful look at the varactor characteristics reveals the optimal switching requirements. If the varactors are switched between ground (zero volts) and the supply voltage ( $V d d)$ then a significant portion of the waveform creates no change in the capacitance of the varactor. The high-slope region thus needs to be exploited and this shifts up the switching voltage from zero to $V_{t h}$ so that switching between $V_{t h}$ and $V d d$ yields the maximum change of capacitance. The smaller the rise time of the switching waveform, the less pronounced the modulation would be at the oscillator output. The VDSM technologies can deliver this sharp rise time as well as small unit varactors so that elements required to achieve small 
changes in output frequency are present. Another refinement is to use a sigma-delta modulator to control the duty-cycle of the varactor control signal in order to gain further precision and granularity. Doing so also ensures that the spurs can be shaped by manipulating the sigma-delta spectrum. A further bottleneck is encountered as individual varactors can generate spurs due to their position in the switching matrix. Hence the switch identity of the varactors needs to be randomized every update cycle. This ensures that varactor-position-related spurs are minimized. With all these modifications, significant performance improvements have been reported for GSM radio chips [12][38].

From the commercial perspective, the DCO:

- brings a digital mindset to the system-level design ensuring repeatability of results, rapidity of development, accuracy and precision in the generation of the required frequency and simplification of radio architectures,

- reduces the NRE cost as the IP can be stored as HDL code,

- reduces spurious output due to the idiosyncrasies of individual varactors,

- allows for automated placement of the varactors as well as the DSP engine,

- allows behavioral simulations of the oscillator using a digital simulator,

- guarantees precise control of the frequency with the best phase noise possible since the gateways of noise have been eliminated,

- guarantees monotonicity,

- allows several modulating signals to be summed up digitally and applied to the final output,

- and opens up the possibility of manipulating digital data to find the spectral content that results in valuable information that can be post-processed.

These advantages are either absent for a VCO or are not feasible to implement. The DCO architecture is a significant and fundamental addition to VLSI design and perhaps rivals the advent of the sigma-delta modulator or the LMS algorithm several decades 
ago. It is also an ideal contender for FPGA offerings tailored for the wireless industry, but to this date a DCO is not found on FPGAs.

\subsection{Loop filters}

Passive loop filters can be integrated on chip albeit at a great cost in terms of area. Generally, capacitor values are limited to $1 \mathrm{nF}$, inductor values are limited to $1 \mathrm{nH}$ and resistor values are limited to several hundred $\mathrm{k} \Omega \mathrm{s}$. High performance thick top metal layers for inductors, special layers for smaller valued Metal-Insulator-Metal (MIM) caps and precision resistors with various specifications are available on most CMOS processes tailored for AMS applications. The response of passive components changes with frequency, PVT variations and is also layout dependent. The integration of passive components has been extensively discussed in the literature over the last two decades.

Up to $20 \%$ of the layout area for a PLL-FS or a CG macro can be used up by the loop filter. Another $20 \%$ is used for the voltage regulator that sports a large capacitor to stabilize the core supply. The front-end terminations take up almost $15 \%$ area to implement two $100 \mathrm{ohm}$ resistors in addition to the ESD devices. Some of this circuitry is indispensable but digital filters can be implemented in a fraction of the space and thus an alldigital system design is incomplete without the inclusion of digital filters. The UP/DN signals from the digital PDs can be filtered using a digital filter. These digital signals need to be filtered to prevent excessive updates of the sampling edge in a CDR system. If the input data suffers from high frequency jitter, but the frequency of the incoming data is constant, then the sampling point does not need to be updated. This improves the high frequency JTOL or the timing margin of the CDR system. If the input data edges are accumulating a slow drift in any direction, the VCO frequency needs to be updated to track the incoming 
data. This is termed as tracking 'low frequency wander'. The evolution of the digital filtering scheme is shown in three steps in Figure 3.20.
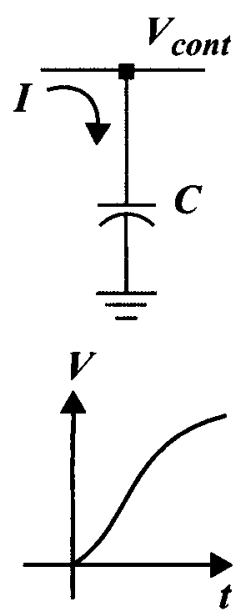

(a) Analog integrator
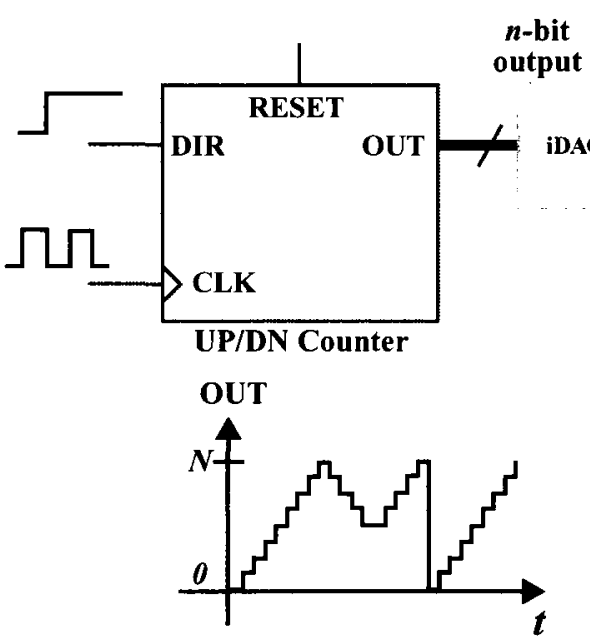

(b) Digital integrator

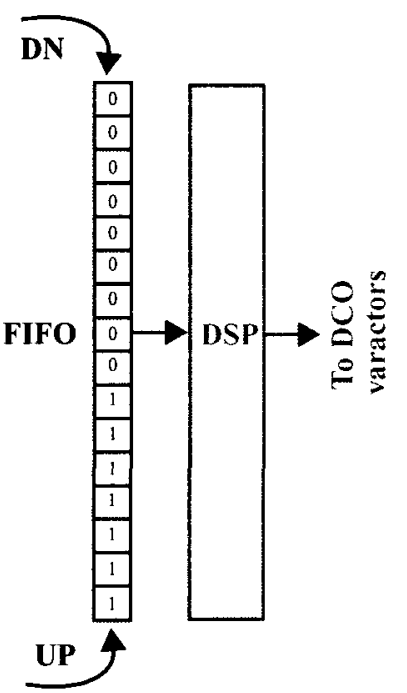

(c) FIFO

Figure 3.20 Loop filter evolution

The digital LPF can be constructed using a digital counter, since a counter is an integrator. This would be the first step in mimicking the old ways of thinking. The integrator is being replaced by another integrator, albeit digital. Such a counter would need an AMS-intensive DAC in order to convert the resulting count into a voltage to control a VCO. If the architecture is all-digital then the UP signal and DN signal could instead populate or deplete a first-in, first-out (FIFO) array with ones and zeros respectively and the resulting thermometer code could be directly applied to varactors to control the DCO. In effect, a FIFO is just like a capacitor being charged by a CP-style charging/discharging mechanism as shown in Figure 3.20 except that a FIFO can be automatically synthesized and laid out using a fraction of the time and area needed for a capacitor. PVT variations do not affect the output signal amplitude of a flipflop significantly, only its speed.

Thus to realize that analog is nothing infinite-level digital — and that such a construct can 'never' be required in practice results in cost-effective system-level solutions. 
This concept will be further developed in conjunction with the discussion on PD modeling in Chapter 4. A survey of CDR architectures is presented next.

\subsection{CDR Architectures}

The following survey of CDR literature is not an exercise in the classification of CDR circuits - and certainly not using components as pivots. Over the decades, many CDR architectures have been proposed to accommodate the increasing data rates in optoelectronic circuits [27][23]. In this chapter, a host of component choices for CDR circuits have been considered. In light of this discussion, it is obvious that the permutation of component choices can result in a large number of options available to the designer - and not all options are created equal. Instead a general trend has been the evolution from purely analog to digitally-controlled analog circuits and toward hybrid architectures that deliver required results. The following survey briefly evaluates the various strengths and weaknesses of the each CDR architecture from different viewpoints. These include but are not limited to design effort, PVT compliance, yield, upgradability, configurability, portability, automation and testability.

A fair question at this point is whether CDR architectures can be classified using algorithms as pivots-say digitally-controlled vs. linearly controlled. The short answer to this question is that if algorithms apply to individual components then the same dilemma would reappear at a later time during the classification since components will respond and deliver the objectives to varying degrees using a particular algorithm. Can architectures be classified using another metric? The answer is that if two components or even algorithms in a system interact and exhibit a trade-off in two different comparative dimensions, then such a classification would be a disguised form of using components as pivots. 


\subsubsection{Reference-less, full-rate CDR architectures}

The reference-less full-rate CDR circuit [22][130] is redrawn in Figure 3.21. It is to be noted that the cited architectures do not use a QVCO but generate the quadrature phases separately. The QVCO inductors and the LF capacitors are area intensive as is the regulated voltage that is required for the system. A possible future solution can be stacking a chip carrying passive and area-hungry elements grown on a specially tailored substrate.

The frequency tracking loop needs to be slow to guarantee stability. The hand over from frequency acquisition mode to phase tracking mode is non-trivial but separating the paths leads to a better design. A logical evolution of this strategy is to sub-divide the phase tracking loop functionality to implement coarse and fine tuning for the VCO along with a separate frequency acquisition loop. This division is driven by the type of modulation that the data stream carries and is not general to all architectures. Due to predominantly analog sub-circuits, the reference-less CDR systems represent the initial R\&D phase of the early 1990s. The legendary purity of the recovered clock can still set a reference point for other architectures due to the continuous PLL. This design is not portable and cannot scale with the bit rate since every individual component has to be substantially redesigned, perhaps with a modification to its particular architecture with increasing speed.

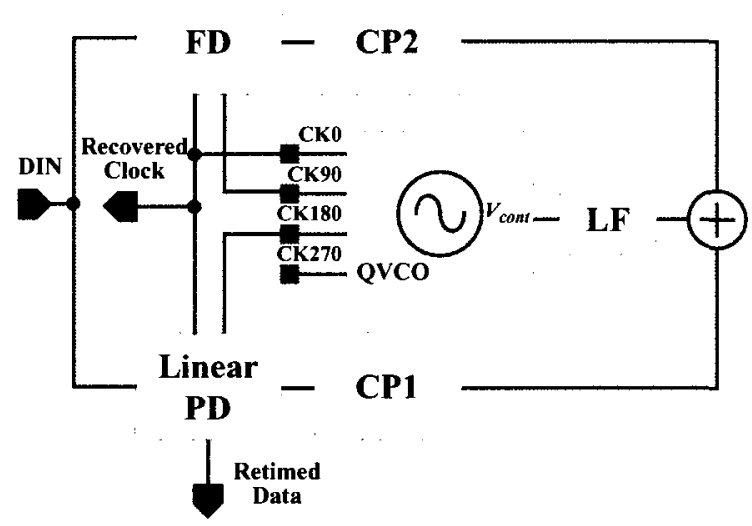

(a)

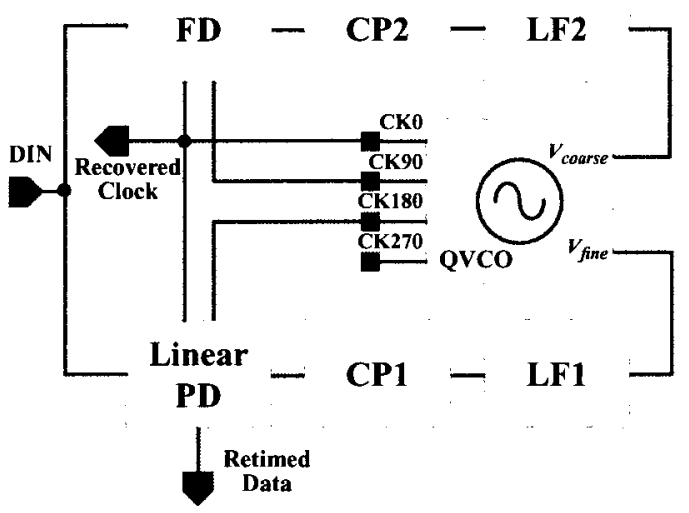

(b)

Figure 3.21 Linear CDR (a) single VCO control (b) dual VCO control 


\subsubsection{Fully-digital CDR systems}

Keeping with the evolutionary theme, two semi-fictitious schemes are explored in this section to highlight what it would mean to have an all-digital CDR system. Assuming that a DCO is available and needs to be integrated in an existing linear design, an ADC can be used to drive this DCO. This is shown in Figure 3.22(a). The front-end and the ADC in this scheme are inefficient and AMS intensive. The delay through the CDR loop reduces the stability and degrades the BER. This is still not an all-digital CDR system but a step in the right direction limited by the AMS complexity and speed of its components. Missing converter codes can create abrupt jumps at the DCO output. Since FPGA systems might offer DCOs in the future, this architecture could be explored in the embedded context as and when it becomes practical to do so. As shown in Figure 3.22(a) with an analog PD, $\mathrm{CP}, \mathrm{LF}$ and a DCO it is a true architectural oxymoron and has been presented for illustration purposes only.

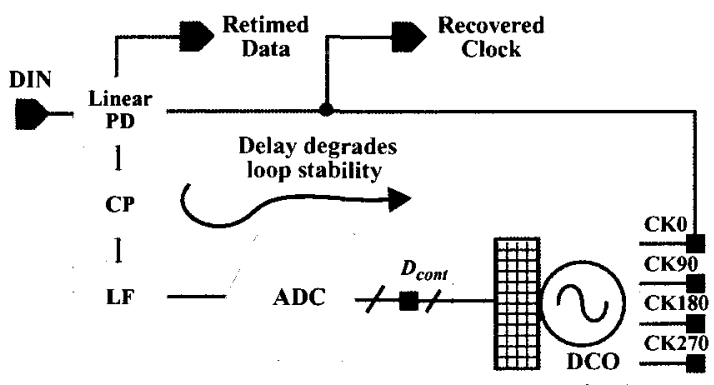

(a)

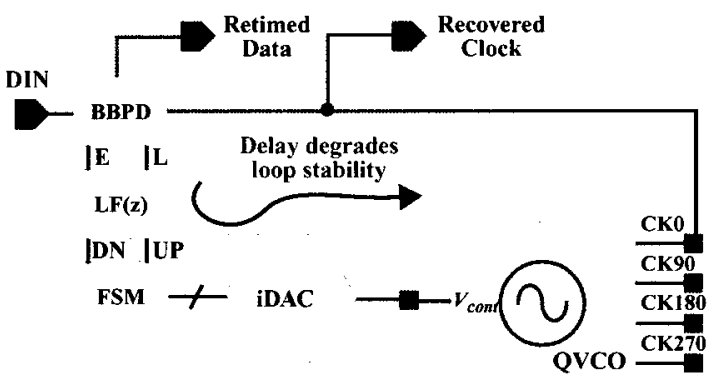

(b)

Figure 3.22 Inclusion of data converters in CDR loops

A converse example is shown in Figure 3.22(b). Here a BBPD provides digital outputs for phase tracking and a digital filter follows the PD. All this is within the purview of currently available technology and tools. This time around a library QVCO is used that requires a continuous input supplied by an iDAC. Practically, this scheme is a less complicated design case due to the iDAC and fine granularity can be achieved at the VCO output using interpolation between DAC codes. This can also be appealing for FPGA platforms 
since $\mathrm{iDAC}$ is a generic block that can be re-used in conjunction with many SerDes blocks. A 'digital' CDR architecture similar to Figure 3.22(b) employing an analog VCO and AMS-intensive iDAC has been reported [57].

It is clear that the data converters add unnecessary AMS complexity to the CDR architecture. A custom or an embedded implementation of a digital CDR circuit would benefit greatly from using a DCO. A practical fact is that DCOs are neither widely understood nor available although that situation is evolving. VCOs and conventional CDR systems are well-understood and are available as custom IP blocks. Hence the DCO forms the desirable portion of Figure 3.22(a) along with the front-end from Figure 3.22(b) that would make the CDR system truly digital, at least from the control perspective. This is shown in Figure 3.23(a) and Figure 3.23(b) for the BBPD and oversampling PD cases. A frequency acquisition loop is present but not shown. A point to note is that the CDR system has no frequency-related bottlenecks and requires a working DCO and working flipflops for its implementation. A truly synthesizable architectural variant could be a Direct Digital FS (DDFS)-based multi-phase DCO CDR circuit; but it would be limited to lower frequencies due to the limitations of the standard cell libraries and synthesis tools.

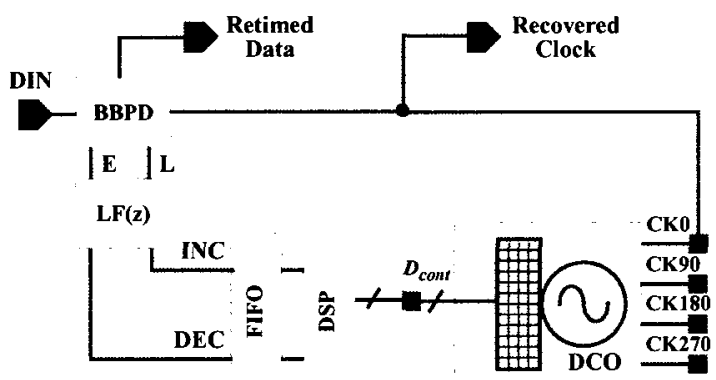

(a) BBPD based

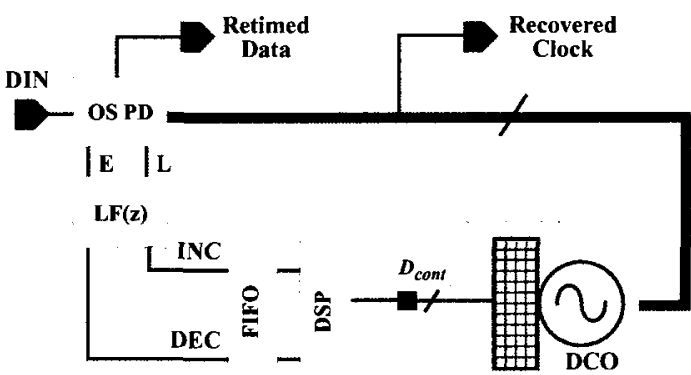

(b) Oversampling PD

Figure 3.23 An all-digital DCO-based CDR architecture

Interim combinations can be explored when understanding, availability or platform choice pose practical challenges. This DCO-based CDR architecture is unexplored in the 
literature as of June 2010 although commercial GSM radios [38] have been reported using

DCO techniques as discussed in Section 3.11. Complex SoCs can benefit from a library DCO as can FPGAs. An electronic oscillator maintains its AMS character on a silicon substrate, regardless of the way it is controlled.

\subsubsection{Full-rate CDR architectures with a local reference}

It is more beneficial to achieve a stable frequency through the use of a local crystal reference as it is not corrupted by the channel. As the CDR circuit locks, the phase tracking can be performed by the selected PD scheme. This opens up more possibilities for clock generation using multi-phase oscillators and DLL based clock generators on the RX side. The benefits of a local oscillator in CDR systems have been discussed in the literature [43][44]. Figure 3.24(a) shows a dual-VCO locking arrangement [58]. The VCO2 and the LF2 generate a control voltage that drives the phase-tracking loop (PTL) to within its tracking range. This also allows independent loop dynamics for the coarse and fine loops resulting in enhanced spectral purity for the recovered clock. However, double the area is required for dual VCOs and LFs and coarse ripple can unlock the fine loop that has a much lower bandwidth.

The coarse frequency loop can be replaced with a programmable iDAC but doing so would void the PLL feedback advantage and increase the programming complexity of the system. Other challenges that have been indicated [27] are frequency pulling that limits the ability to accommodate spread spectrum clocking (SSC), extra area for filters and matching of VCO1 with VCO2. Multiple lanes do benefit from the separate CG PLL.

The sequential locking CDR architecture [22][111] of Figure 3.24(b) is more suitable for a multi-protocol SerDes due to competitive specifications for area and power. To reduce the hardware, a LD circuit is employed that switches control from the frequency tracking loop (FTL) to the phase tracking loop (PTL). The handover from FTL to PTL can potentially unlock the CDR circuit and this problem must be carefully studied over cor- 
ners. The architecture in Figure 3.24(b) is conceptually simpler and modular. The FTL can be progressively shut down during a hand over. Moreover, the FTL can be developed in parallel with the PTL.

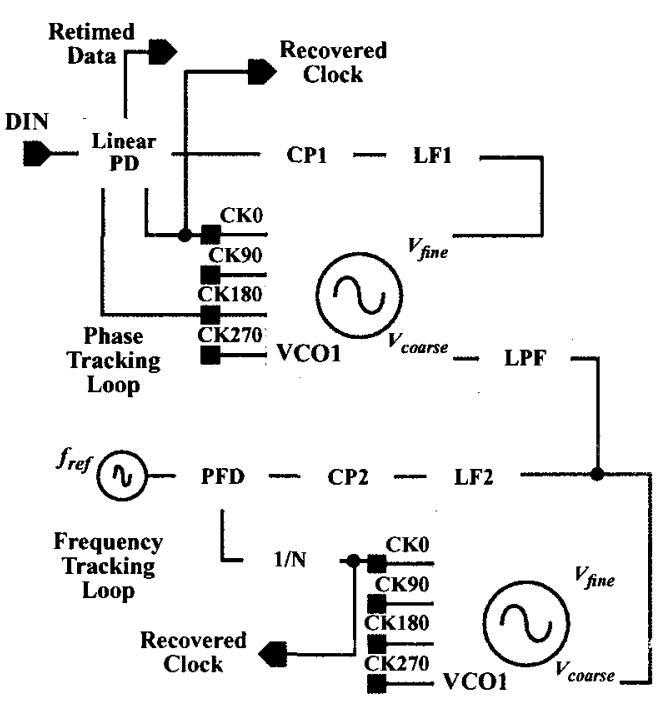

(a) Dual VCO, dual loop

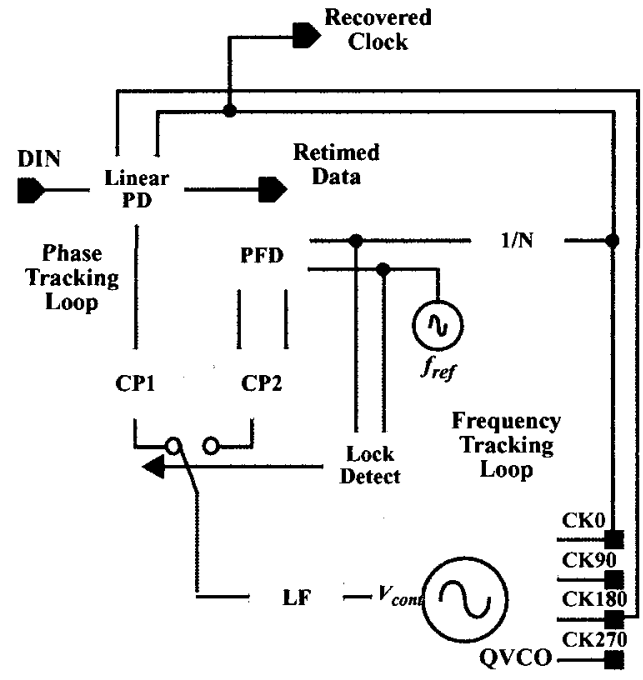

(b) Conventional linear CDR system

Figure 3.24 CDR architectures with a local reference

In keeping with the evolutionary theme, a DCO can replace the VCO along with a digital PD as shown in Figure 3.25(b). The reader has already noticed that the design of the CDR system will be done in the linear domain [133] as long as a continuous VCO is present in the system. As soon as the VCO is replaced with a DCO, a discrete-time analysis becomes more feasible [57].

\subsubsection{Full-rate binary CDR architectures}

To implement a conventional binary CDR circuit, a binary PD replaces the linear PD in the PTL as a minimum requirement [42] as shown in Figure 3.25(a). A good discussion of trade-offs for linear vs. binary CDR systems is present in the literature [134] but it predates the use of a DCO, so a binary PD vs. linear PD is emphasized therein. An analysis of BBPDs based on sigma-delta modulation has been reported [47]. 
The loop as a whole does not become digital. The same legacy analog design problems remain but the design methodology is widely understood. Figure 3.25 (b) shows an all-digital approach that builds up on the all-digital CDR architecture previously shown in Figure 3.23 by adding a DCO block. It is possible ot analyze the system shown in Figure 3.25(b) in the discrete-time domain [57]. The LD circuit with a multiplexer chooses between the FTL and the PTL. This architecture can be augmented to provide oversampling functionality as depicted in Figure 3.23(b).

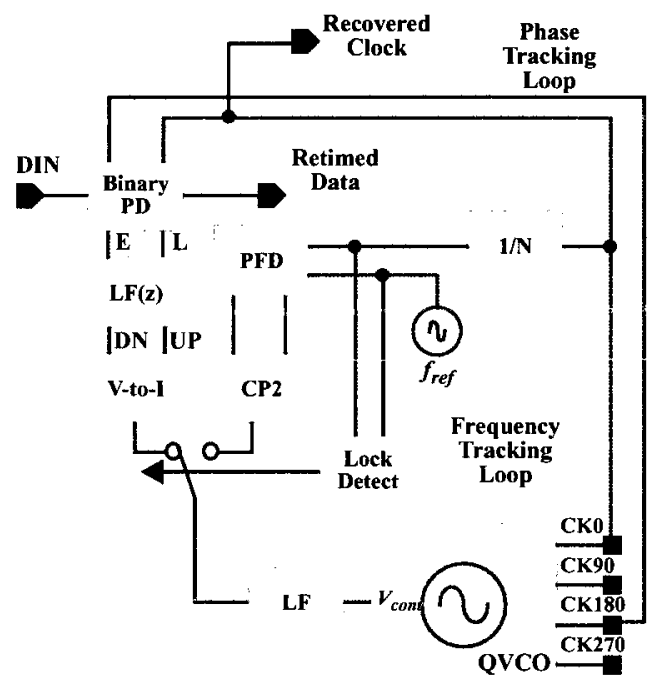

(a) Conventional binary CDR

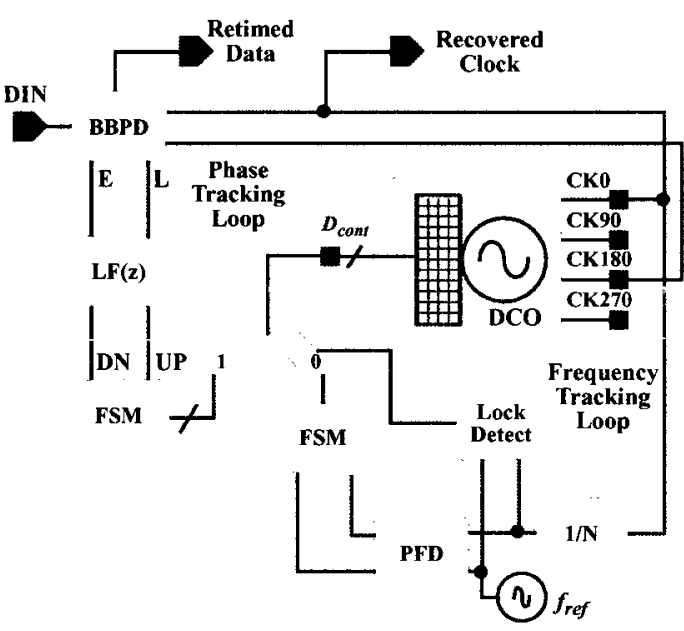

(b) A possible full-rate, all-digital CDR implementation

Figure 3.25 Full-rate, binary CDR architectures 


\subsubsection{Reduced-rate CDR circuits}

The limitations of full-rate architectures-linear or binary-have been discussed in Section 3.7.7. To ease the flipflop speed requirements, the full-rate architectures can be converted into half-rate linear [99] or half-rate binary [100][105] architectures.

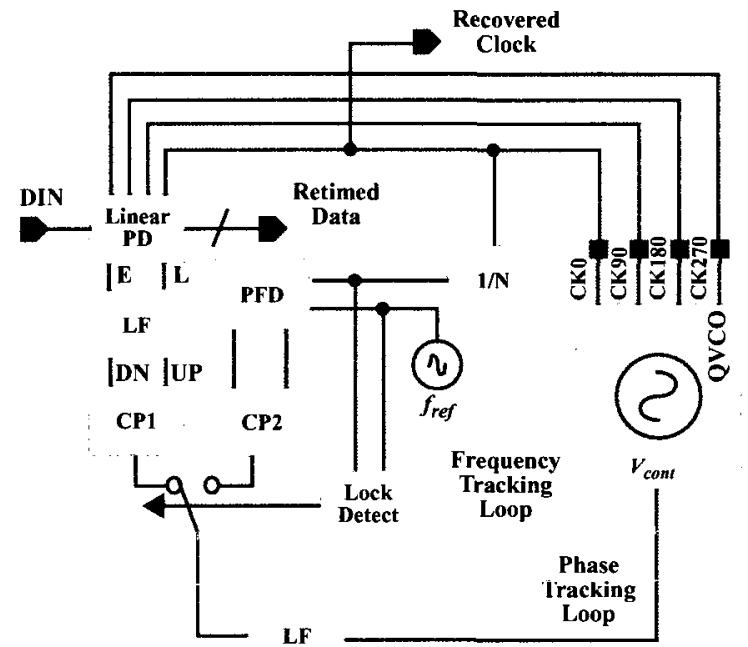

(a) Reference-based linear, half-rate CDR architecture

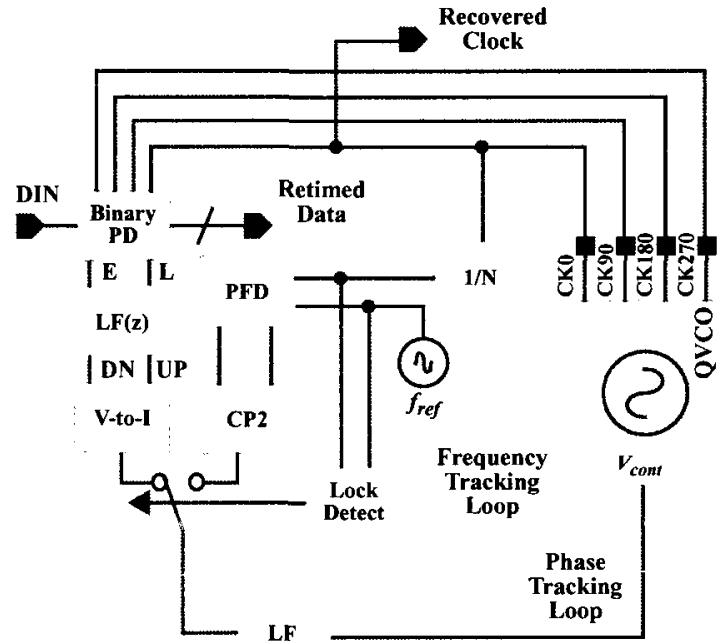

(b) Reference-based binary, half-rate CDR architecture

\section{Figure 3.26 Half-rate CDR architectures}

The inter-phase spacing of an oscillator stage is not a bottleneck since digitally controlled phase interpolators (PI) can always provide finer granularity. It is the variance of the delay that limits the performance. As an example, if a $10 \mathrm{~Gb} / \mathrm{s}$ CDR system requires an 8-phase ring oscillator running at $1.25 \mathrm{GHz}$, then the phase spacing is 125 ps per phase. If the delay spread for the technology is 25 ps over PVT corners, then the worst case is 137.5 ps that corresponds to $9.09 \mathrm{GHz}$. Hence a more advanced process node would have to be used for this design problem. Quarter-rate architectures using hybrid techniques for generation of multiple phases have been reported recently [56]. 


\subsubsection{DLL-based CDR circuits}

A DLL can be used to recover the incoming NRZ data [89][96]. Figure 3.27 shows an evolved usage of a voltage-controlled delay line (VCDL) that allows the decoupling of JTOL and JTRAN for CDR systems [90]. A wide bandwidth FTL loop would have delivered a wider JTOL bandwidth but a degraded JTRAN performance with associated jitter peaking. Instead, the extraction of a clean clock from the narrowband PLL results in independent JTRAN and JTOL optimization. The JGEN is also limited by VCO phase noise.

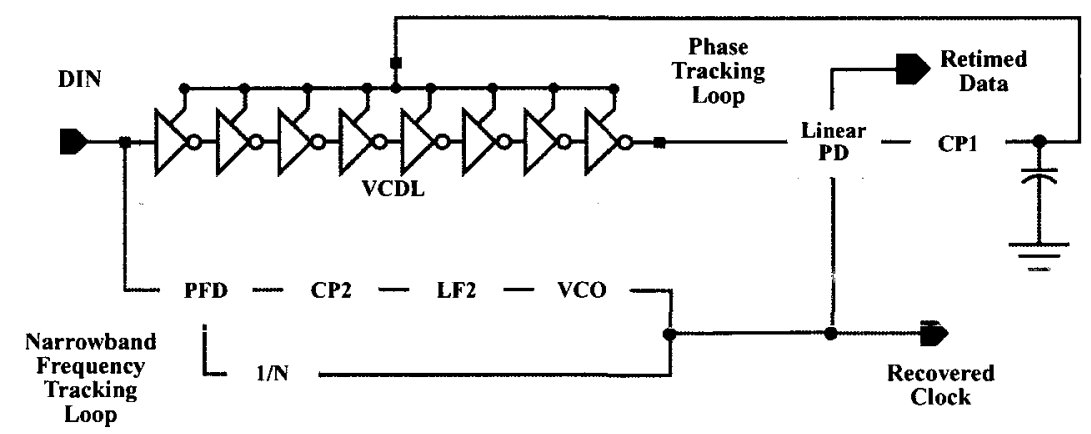

Figure 3.27 CDR system with a wideband DLL with narrowband PLL

Figure 3.28 shows a D/PLL-based CDR circuit that is suitable for multi-protocol SerDes applications with bit-rate detection circuitry (separate loop, not shown here) while offering independent JTRAN and JTOL optimization [91]. This implementation also saves the area for the extra DLL capacitor and has reported better than SONET performance. Hence the judicious inclusion of a DLL provides calculated architectural benefits [27].

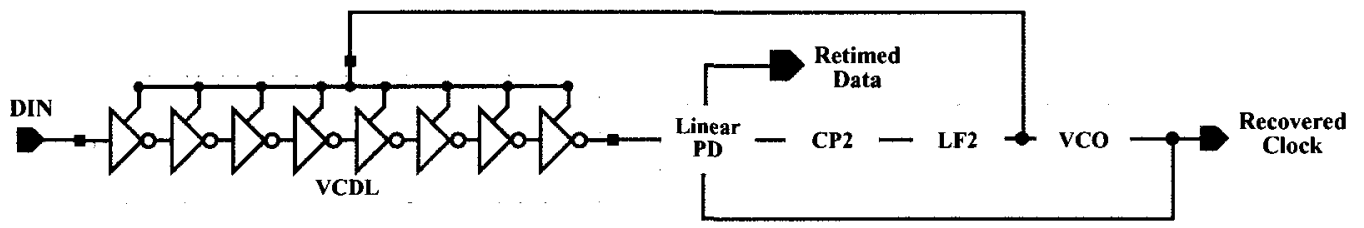

Figure 3.28 DLL/PLL based CDR

A DLL provides multiple phases each of which can be positioned at the centre of the data bit as the sampling clock. This requires a phase rotator circuit. If the phase spac- 
ing is not sufficient then PIs can be employed. For clock recovery, an interpolator would be required to smooth the cycle-to-cycle phase jumps.

\subsubsection{Phase-selector CDR circuits}

The technique of phase selection predates the use of DLL in CDR circuits. Phaseselector CDR variants are shown in Figure 3.29 [60]. These use a multi-phase VCO and select the appropriate phase using a clock phase pointer (CPP). Choosing a phase directly and using it for CDR operation as shown in Figure 3.29(a) causes a discrete phase jump at the clock output determined by the inter-phase resolution. This may be good enough for data recovery (DR) operation. With a subtle architectural change and without requiring analog PI circuitry the VCO can be steered slowly to the new frequency as shown in Figure 3.29(b). The selected phase is fed back and one of the phases is directly used reducing the recovered clock jitter essentially reducing peak-to-peak jitter that would have dominated the total jitter (TJ). The phase selector architecture is digital in nature as far as the PTL is concerned. A multi-phase DCO would morph this architecture to Figure 3.23(b) where a phase selector was not shown purposely for the clock being recovered. A DLL could also provide multiple phases but with a reduced frequency range.

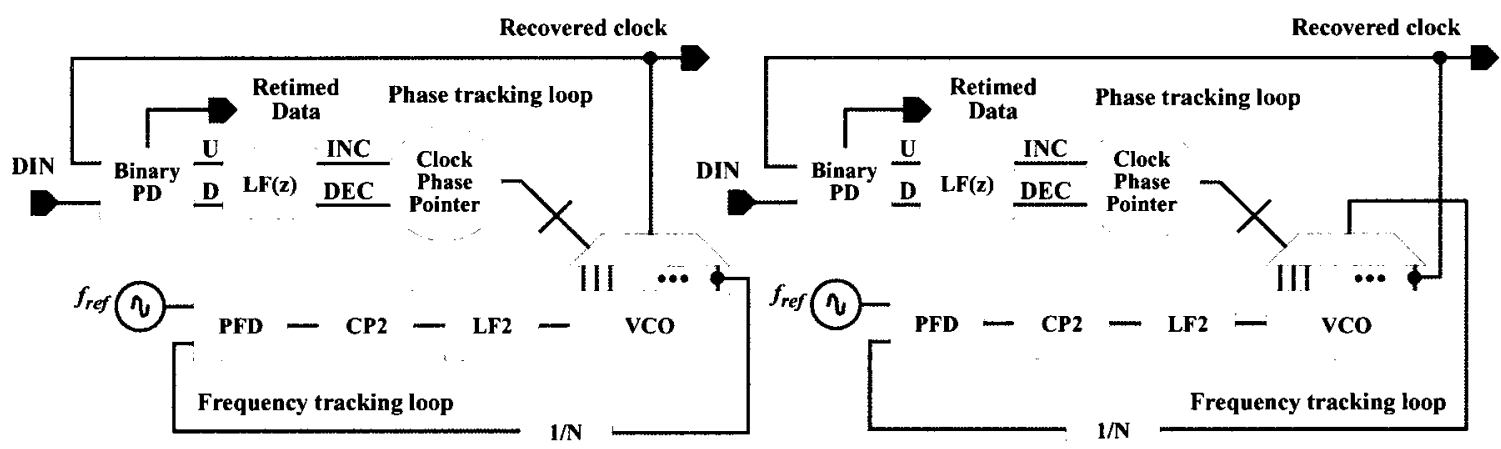

(a) Phase-selector CDR circuit

(b) Feedback phase selection

Figure 3.29 Phase-selector CDR circuit 
A modification of Figure 3.29(a) that uses a Phase Interpolating (PI) circuit following phase selection has also been proposed [61][98] and is shown in Figure 3.30.

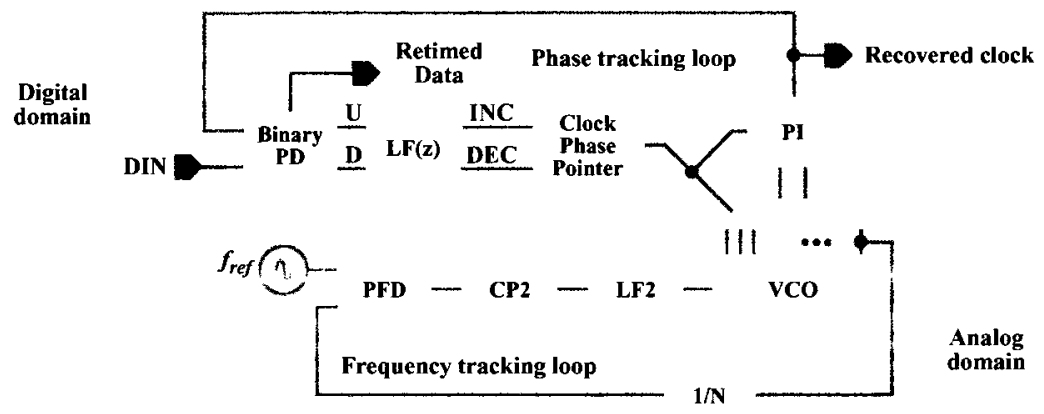

Figure 3.30 Phase-interpolating CDR architecture

At first glance, it seems a bit inferior than the more AMS-intensive feedback-phase version of Figure 3.29(b), but the advantage of using the PI-CDR architecture lies in the possibility of digitally controlling the recovered clock phase in fine increments without using a DCO. The drawback is the added, but manageable, complexity introduced by the PI circuit. Indeed a DCO was not as well-known when a PI-CDR architecture was proposed which makes this architecture quite elegant.

\subsubsection{Injection-locked CDR architectures}

An injection-locked CDR (IL-CDR) architecture [95] is shown in Figure 3.31. This is a variant of the PI-CDR.

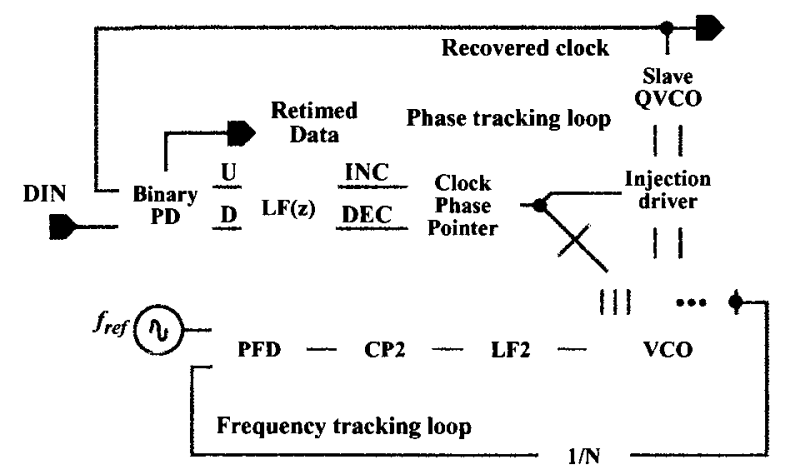

Figure 3.31 Injection-locked CDR Circuit 
Due to the chaotic analytical nature of injection locking, it is hard to analyze the behavior of the loop especially at start-up. The slave oscillator provides a better duty cycle balance. The IL-CDR architecture is AMS intensive.

\subsubsection{Burst-Mode gated-oscillator CDR architectures}

A burst-mode gated oscillator CDR (GO-CDR) architecture is used in burst-mode passive optical networks (PON) [106][107] and is shown in Figure 3.32. It offers fast synchronous CDR operation for fiber optic links.

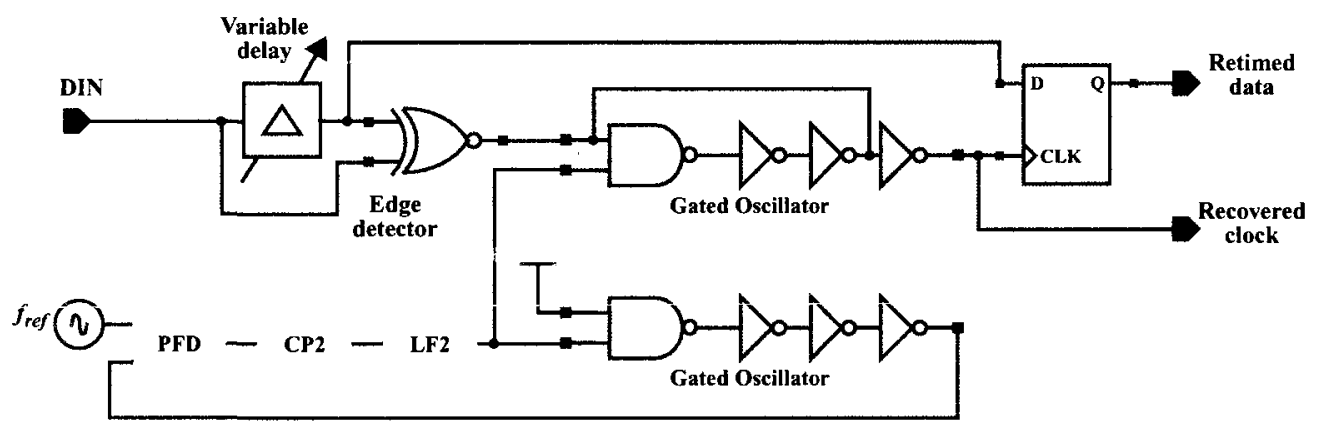

Figure 3.32 Gated-oscillator CDR circuit

The clock is derived from the gated-oscillator triggered by an edge detector. The frequency of the tuning oscillator is regulated using a replica ring oscillator with its gated input tied to logic high. The extra NAND gate ensures similarity between the two oscillators. The architecture has a wide bandwidth due to no LF being present and this degrades the JTOL. The advantage is a small footprint, power and the ability to receive data packets after a burst of training pulses that is common in PON applications. Recently, a gated ring oscillator with high performance has been reported that can provide 6 ps of delay resolution and consumes small area [77].

The GO-CDR is an area of active research in optoelectronic IC design and nothing short of a full-length review would be required to do it justice [108][109][110]; the space or the scope do not permit it here. 


\subsubsection{Jitter tracking CDR architectures}

Up until now, the CDR architectures discussed have used conventional PLL-style tracking. Jitter asymmetry and DCD can degrade BER for these architectures. The sampling point is not always at the visual center of the data eye - except for the case of symmetric jitter on both edges. The solution is to track the asymmetrically jittered data edges and use a sampling clock that is situated midway between the tails of the jitter distributions on either edge. This ensures the lowest BER performance. The concept is depicted in Figure 3.33.

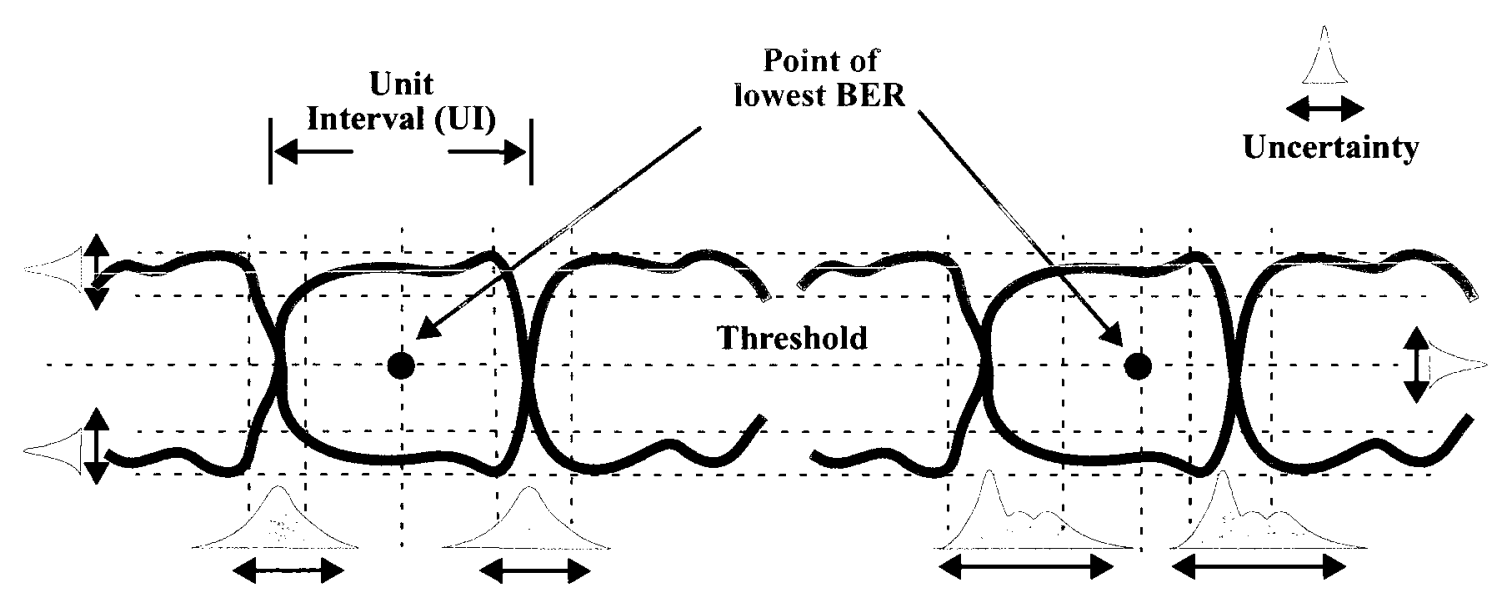

(a) Symmetric jitter

(a) Asymmetric jitter

Figure 3.33 Effect of asymmetric jitter on CDR operation

A minimum of three edges are required for this funcationality giving rise to the $3 x$ oversampling (3XO) CDR architecture [97]. If continuous tuning is employed for jitter tracking edges, then such a variable interval oversampling architecture becomes an AMSintensive project that is non-portable. The three edges can also be derived from a multitude of oscillator phases and can have coarse and selectable granularity imparting a more digital outlook to the CDR architecture. 


\subsubsection{Variable-interval $3 x$-oversampling CDR architecture}

The variable interval, $3 \mathrm{x}$-oversampling, mixed-signal CDR (VI-3XO) CDR architecture is redrawn from the original publications [49][50] to emphasize the various tracking loops. Figure 3.34 shows the details of the VI-3XO architecture.

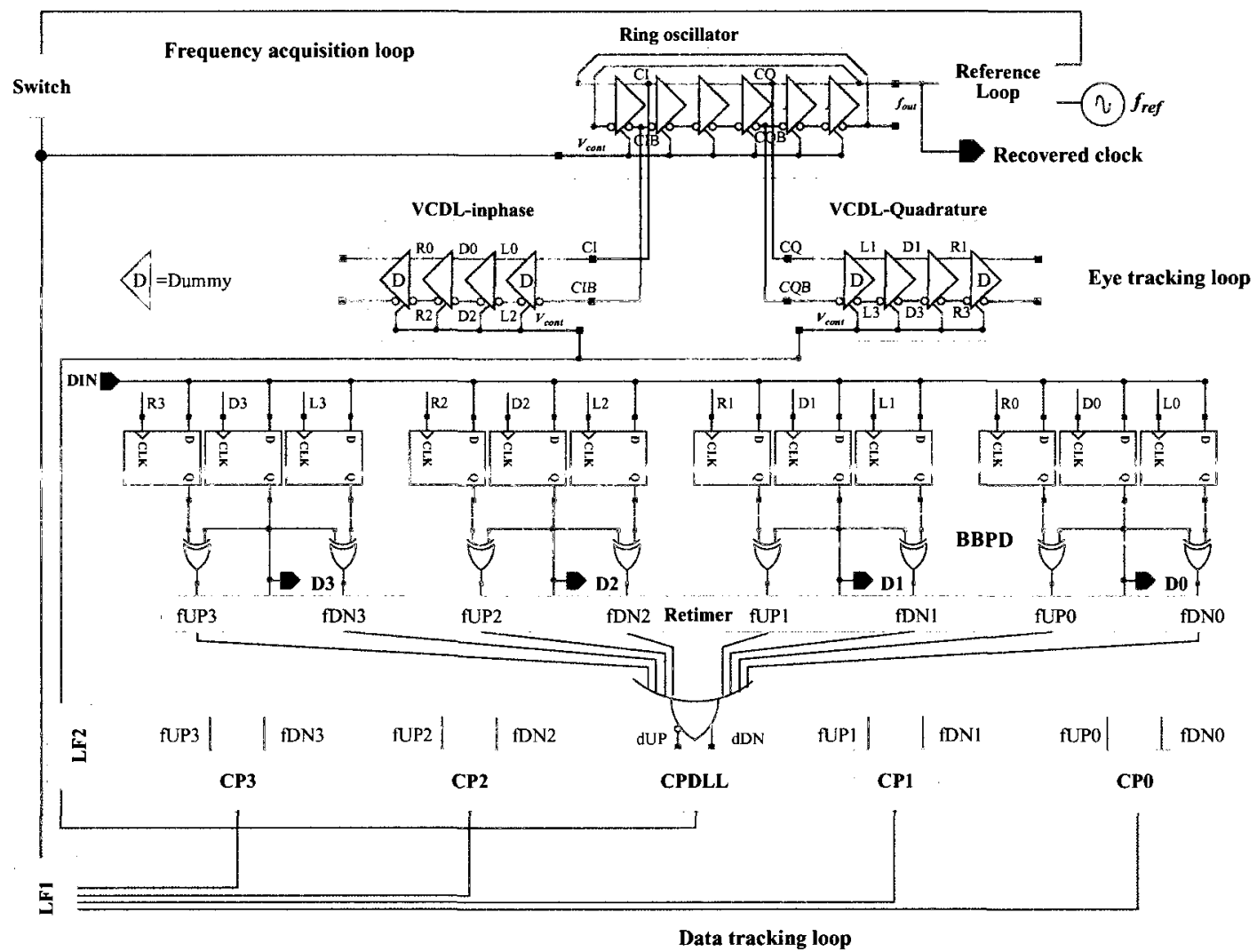

Figure 3.34 Variable interval oversampling CDR architecture

This architecture uses a quadrature clock at $1.25 \mathrm{GHz}$ to provide CDR operation at 5 Gbps. A ternary PD quad operates on four data bits simultaneously to collect jitter information. A frequency tracking loop locks to the incoming data frequency. Then the tracking loop and the eye-measuring loops become independent of each other and the DLL provides three edges per UI to track the asymmetric jitter. The centre edges are used to sample the data. The delay cells in the DLL and the ring oscillator are identical. Interaction(s) between the acquisition loop, the tracking loop and the eye-measuring loops are to be min- 
imized for achieving the desired stability over PVT corners. In short, many CDR components and strategies discussed until now have been used in this VI-3XO CDR circuit.

The AMS complexity of the VI-3XO CDR design renders it non-portable without significant effort. The bottlenecks in this architecture are the capacitance at the input data node, area required for the two filters, the switchover between the acquisition and tracking loops, the matching between VCDL and oscillator stages, biasing arrangements and multitudes of clocks sharing the same layout area creating crosstalk in addition to potential oscillator pulling. The VI-3XO CDR architecture provides a measure of the horizontal eye-opening and this information can be creatively used at various points in the RX, especially the DFE. The horizontal eye opening measurement is a reusable technique that can be employed in conjunction with other architectures.

\subsubsection{All-digital eye-tracking DR architecture}

The eye-tracking, 3x-oversampling DR (ET-DR) architecture is also based on the concept of tracking asymmetric jitter on data edges. The ET-DR [48] is shown in Figure 3.35. Compared to the VI-3XO CDR architecture, it is a DR architecture only and uses a coarse selectable phase using a clock phase pointer (CPP).

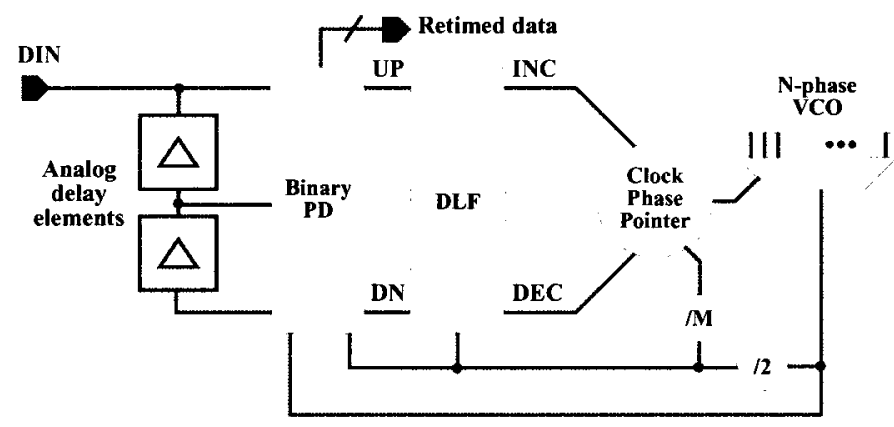

Figure 3.35 All-digital eye-tracking DR architecture

The incoming data is delayed twice using tunable analog delay elements thus creating three copies of the data spanning a part of the UI that can be thought of as a sampling aperture for 3XO PD operation. The sampling clock is chosen from the ' $N$ ' available clock 
phases and is rotated in either direction to track the data eye at a frequency $f_{c l k} / M$. The inter-phase spacing in this architecture is fixed compared to the VI-3XO CDR architecture. The CPP is driven by a BBPD and a digital filter. The analog elements to be designed are the front-end flip-flops, the analog delay elements and the oscillator core circuitry. This ET-DR architecture has demonstrated a high-frequency jitter tolerance of 0.7 UI in the presence of asymmetric jitter for a SFI-5 backplane with sixteen lanes at 2.5 Gbps each, while providing data recovery at a BER of $10^{-12}$ [48]. The increased throughput and the digital core makes the ET-DR portable with the exception of the multi-phase oscillator and the analog delay elements.

\subsubsection{Blind Oversampling CDR}

Oversampling techniques were well known at the time when these were employed by Motorola ${ }^{\circledR}$ during the early 1970 s in conjunction with a serial universal asynchronous receiver/transmitter device. For very low frequencies, it was possible to implement $16 \mathrm{x}$ or $32 \mathrm{x}$ oversampling. How far the sampling point was from the edges could be determined with redundant granularity and accuracy; however, the generation and routing of multiphase clocks becomes challenging at high frequencies.

A blind oversampling (BOS) CDR circuit works when two fundamental assumptions about the data and the sampling clock are observed.

- The incoming data is at the same mean frequency although its phase can drift due to the jitter acquired in the channel.

- The sampling instances are uniformly distributed.

This architecture differs from the PLL-style architectures, since there is no apparent feedback connection and charge-pump/filter combination. It simply relies on deriving signal statistics based on a finite number of samples over a time window and then making the bit decisions based on an algorithm. The coarse phase spacing resolution employed by 
the CDR circuit is not the main performance limitation [51]. The block diagram (adopted from [51]) is presented in Figure 3.36.

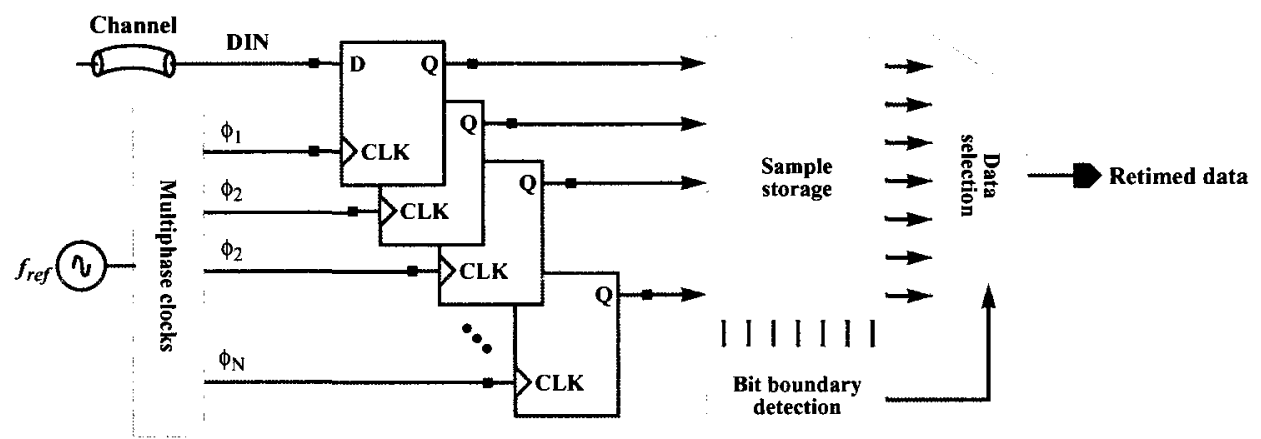

Figure 3.36 Blind oversampling CDR block diagram

A serial NRZ stream is sampled using a bank of flipflops (also called samplers) and a non-synchronized reference clock supplying the required oversampling phases. These data samples are then stored into a memory. The bit boundaries may be clearly defined for a repeating $101010 \ldots$ pattern, but in case there are long sequences of ' 0 ' and ' 1 ', the bit boundaries can be extrapolated using digital logic. After the bit boundaries have been found, the data can simply be selected as the sample that is farthest from the edges. An odd OSR removes the ambiguity while making this selection and is, therefore, employed. Another method for data selection could be a majority voting scheme, but research has shown previously that the centre-picking results in better performance compared to majority voting [52]. The reason is intuitive. At relatively high frequencies of operation, it is not possible to have a glitch at the centre of the eye due to $f_{\tau}$ limitations. Hence the sample between the edges is more likely be the correct sample. The same work [52] also demonstrates a "phase-picking" architecture at 6.4 Gbps using a $0.35-\mu \mathrm{m}$ CMOS technology. The power consumption is $600 \mathrm{~mW}, 60 \%$ of which is spent in the FIFO. This FIFO area is not a concern for modern processes but the power situation does not change if thousands of bits are to be saved at speed. Still, the all-digital and the algorithmic nature make it a viable choice due to its portable outlook for low frequency applications for FPGA platforms. FPGA vendors, however, are better off with the DCO route. 
The BOS CDR architecture is a departure from all other CDR architectures before it. The focus has, until now, been on recovering a clock from the data stream and then using this clock to sample the data stream using only a single flip-flop. For the BOS CDR, the ever-changing data is being stored (in thousands of flipflops) and the clock edge that gives the best results is being chosen afterwards. In other words, the constancy associated with the clock frequency is not being exploited. Thus, a clock-centric focus for CDR design is efficient whereas a data-centric focus is wasteful of area and power.

The BOS architecture makes tacit assumptions about the speed of the available technology. First, the synthesis tools put an upper limit on the frequency of the synthesizable blocks. A higher $O S R$ can only be implemented if the technology allows it. The number of samples that have to be saved in order to make a decision on what constitutes the optimum sampling point requires a large number of flipflops. Saving the samples does not result in much benefit unless statistical post-processing needs to be done. In a stealthy manner, this is nothing short of a DSP engine determining where the edges are falling so as to be able to control the sampling point - in other words, a power-hungry algorithmic PLL. The lesson is that all-digital and portable may not always the best choice.

A hybrid between a BOS DR and a phase-tracking CDR circuit has been reported whose JTOL is the multiple of the JTOLs for a phase-tracking CDR and a BOS DR [53]. A particular thing to note in this publication is the saturation of the JTOL characteristics for the BOS DR as the FIFO gets saturated. A similar effect would be evident in a phaserotating $\mathrm{CDR}$ architecture that is limited by the update period unless frequency acquisition circuitry is employed. A power efficient BOS DR has been reported on an FPGA platform that has explored tracking algorithms in some detail [54].

\subsubsection{Multi-level CDR circuits}

The pulse amplitude modulation (PAM) using one symbol per UI is known as PAM-2 or NRZ signaling. An extension of the idea leads to PAM-4 or two symbols per UI 
using four discrete levels. This does not provide much better results than the PAM-2 case due to the equalization power required to achieve the specified BER. This was used in conjunction with memory architectures around 2003, and publications in the open literature have since then dried out [117][118][119][120]. Due to the AMS-intensive nature of such CDR architectures, these have not been pursued further in this work.

\subsubsection{Multi-valued DR architectures using CDMA techniques}

A multi-valued CDR circuit using Code Division Multiple Access (CDMA) techniques was introduced but was limited by the frequency of CDMA style 'chips' used for data recovery [121]. The idea is eclectic, novel and perhaps ahead of its time. Equalization techniques for multi-valued DR techniques along with inductively-coupled wireless interchip data transmission has been reported [122]. BPSK modulation has been used in this work as reported earlier in the literature [124]. For wireless inter-chip SerDes on SiP modules, the multi-valued DR architectures could become important in the future where a wider optical bandwidth could change the balance in the favor of CDMA-over-DWDM style CDR architectures.

\subsection{Jitter in SerDes}

This section reviews the basics of jitter ${ }^{1}$ in the SerDes design context. Jitter puts an upper limit on the performance of SerDes hardware. Many design and verification challenges arise as a result of jitter. The instrumentation and techniques used to characterize jitter remain an area of active research and the literature remains distributed in technical journals with notable exceptions [9].

1. Etymology of jitter is not precisely known and the first usage is 1929 according to Meriam-Webster online dictionary. Oxford English Dictionary Online (OED-Online) defines 'jitters' ( $\mathrm{pl}$.) as 'a sense of panic or extreme nervousness' or 'a feeling or raw nervousness'. Also jitter is described as the 'slight, irregular variation in an electrical signal'. 


\subsubsection{Jitter in electronic circuits}

Jitter denotes the temporal non-idealities in an otherwise ideal signal. Jitter in electronic devices may not be limited to either the slight or the irregular variety. Technically, the ITU-T G-810 Recommendation defines clock jitter as the "short term deviation in the significant instants of digital signals referred to their equidistant nominal instants" (as quoted in [151] along with a discussion of the shortcomings of the suggested method). In mathematical form, and assuming a sinusoidal signal,

$$
X(t)=A \sin (2 \pi f t+\Phi(t))+n(t)
$$

where $n(t)$ is the instantaneous amplitude noise and $\Phi(t)$ is the phase modulating waveform that produces the jitter with a defined probability distribution function (pdf). A clock signal has rising and falling edges every cycle. Due to the statistical nature of the noise and the modulating signal itself, a jitter histogram is used to study the types of jitter embedded in a given measurement since averages derived over time produce a clearer statistical picture of the signal's history. 


\subsubsection{Jitter modeling}

It is customary to model jitter as the deviation of edges of an otherwise ideal clocking source. Figure 3.37 shows the various components of jitter being modeled as deviations from an ideal VCO output.

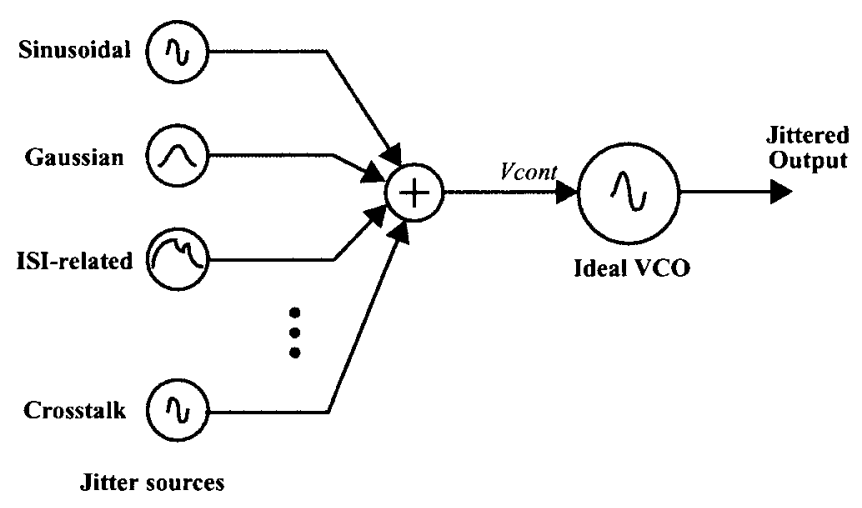

Figure 3.37 Modeling jitter components

Each component in the jitter degrades the BER differently and a single number for the BER is specified in the presence of various kinds of jitter. As a simulation strategy, a testbench involving separate jitter sources is more expedient. The non-trivial challenge is to dissect the jitter components [152] at the receive side that are embedded in the received signal. 


\subsubsection{Classification of jitter}

Jitter is classified as comprising of its sub-components. Figure 3.38 shows the classification of jitter as shown in the Agilent's jitter poster [157].

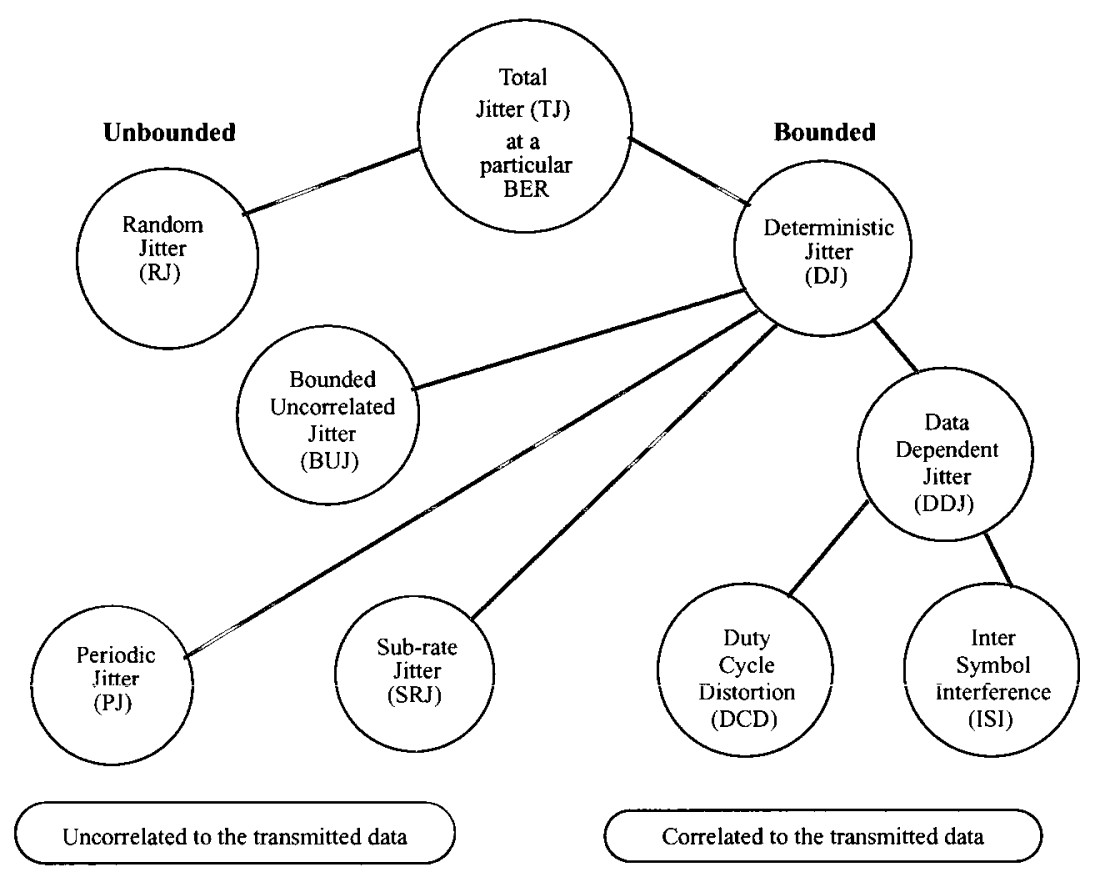

Figure 3.38 Classification of jitter

\subsubsection{Measuring jitter}

Jitter characterization is non-intuitive and requires data storage and post-processing. A host of sophisticated mathematical techniques, CAD tools and measurement instruments give a true picture of the problem at hand. The remainder of the chapter briefly reviews jitter modeling and simulation in the context of the digital CDR circuits.

Characterizing jitter is a complex measurement since the data have to be stored for post-processing. An isolated transition is just as useless as a terabyte of unanalyzed data. Owing to the nature of jitter, a statistical measurement done over a long duration is sought. Watching the waveform on a normal oscilloscope reveals not much about the jitter and seldom benefits the SerDes test and debugging operations. Ordinary scopes can only be set to high persistence to see the eye closure. Sampling scopes with phase and amplitude histo- 
gram functions are used to get more insight into the jitter. Even this does not go far enough. More recently, sampling scopes have started separating different jitter components using math software installed on the scopes. Such a breakdown of jitter results in advanced debugging and verification strategies for serial links. Different instruments from different vendors seldom agree $100 \%$ on the final results due to slightly different jitter separation algorithms. For measurements like JTOL, JTRAN and JGEN, jitter has to be generated according to specifications and subsequently the BER is recorded for different jitter amplitudes. Automated BER Testing (BERT) systems go a step further and scan the eye while maintaining an error count. This data can be fitted to an exponential model to extrapolate down to a BER level of $10^{-15}$-something that is not possible by direct measurement [154].

A confidence level in the measurement determines the time required for a direct test if a BERT scan instrument is not available [155].

\subsubsection{Eye diagrams and histograms}

If a modulated clock is used to drive a PRBS generator, the data waveform shows eye closure as shown in Figure 3.39 (b).

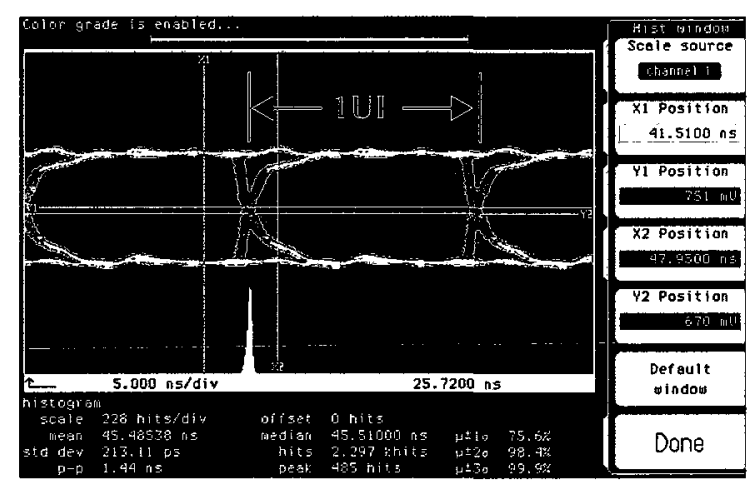

(a) $50 \mathrm{MHz}$ data output with no modulation and residual random jitter

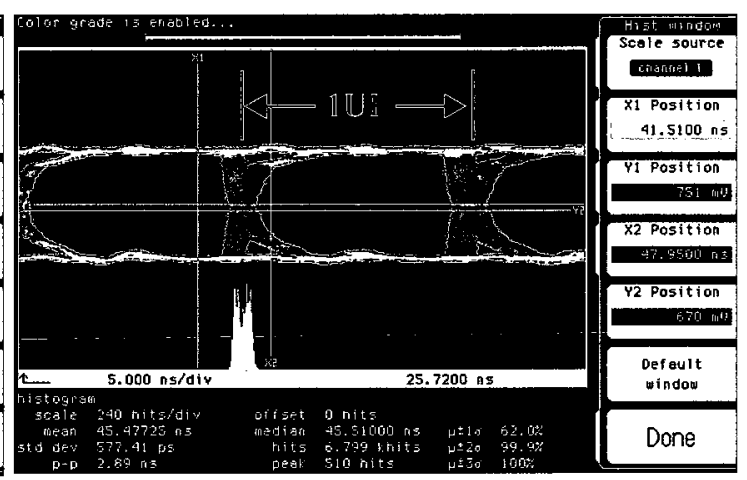

(b) $50 \mathrm{MHz}$ data output with FM 1.0 MHz deviation (a) $1 \mathrm{kHz}$

Figure 3.39 Eye Closure (Signal Integrity Deficit) 
The jitter histogram depicts the probability of the data edge being in a certain location. The probability is the highest at or near the ideal crossing point along with a random (Gaussian, RJ) or deterministic (DJ) jitter added to the clock. Data must be recovered by the CDR circuit with the specified BER in the presence of horizontal eye closure that is caused by specific types and quantities of jitter. If the eye is vertically closed as well, then a DFE is used to open the eye prior to establishing the sampling point.

\subsubsection{RMS and peak-to-peak jitter}

The peak-to-peak jitter is caused by random noise and is unbounded. Therefore, it is dependent on the size of the acquired data. The rms value for jitter applies to a Gaussian distribution such as RJ but cannot encompass all types of jitter. The DJ can be further divided into many jitter components and cannot be related to the rms value in a repeatable manner. Hence more complicated algorithms are needed to separate the jitter and to understand its influence on the BER [153].

The situation is different from the case of oscillators, where RJ (pp and rms) specifications are considered sufficient. There is some fuzziness on how the spurs are specified but spurs are attributable to sources mostly external to the oscillator, e.g., power supply, or substrate or CP noise etc. These are specified as being a certain number of dBs down compared to the carrier. For serial links the final indicator of performance is the BER, i.e., the ratio of erroneous to successfully received bits. The specified values range from $10^{-12}$ to

$10^{-14}$ for backplane applications. Such challenging specifications have implications for simulation, design, prototyping, laboratory testing and production testing as discussed next.

\subsection{Clock and data recovery circuit specifications}

This section presents commonly encountered CDR circuit specifications. It also sets up the stage for the practical problem of simulation of CDR systems. The perfor- 
mance criteria for CDR circuits are mostly set by the standard committees for various applications.

\subsubsection{Bit Error Ratio (BER)}

This refers to the ratio of the incorrectly recovered bits to the number of transmitted bits. For instance, if one bit out of every billion $\left(10^{12}\right)$ bits is received in error, then the BER is simply $10^{-12}$. Since this is a ratio, it is dimensionless.

Often, it is mistakenly referred to as the "Bit Error Rate", and the same abbreviation is used. In doing so, a 'per second' unit should also be appended to avoid confusion. This terminology has carried over from the classical communications theory. The practical difference is that where $10^{-4}$ is considered a good BER for wireless systems, a $10^{-12}$ is usually required for wireline serial links. For a SerDes system, any change to the CDR architecture, its components, the DFE structure or the equalization algorithm results in a change in the BER. Hence it is a key specification. Measuring BER requires a thorough understanding of the types of jitter that can inflict a BER penalty.

\subsubsection{Bit rate, symbol rate and throughput}

Baud rate (or symbol rate) is the number of symbols transferred every second. For binary NRZ signaling, the symbol rate and bit rate are identical. If multi-level signaling is used e.g., 4-PAM then two bits are transferred per symbol and the bit rate is twice the symbol rate. Such an exploitation of signal amplitude is limited by the effectiveness of the equalizer. By far the dominant technique is NRZ signaling also known as 2-PAM.

The data rate of the CDR circuit is the symbol rate times the number of bits per symbol that can be transmitted while maintaining an acceptable BER (usually lower than $10^{-12}$ ). It is measured in either Megabits per second (Mbps or $\mathrm{Mb} / \mathrm{s}$ ) or Gigabits per second (Gbps or $\mathrm{Gb} / \mathrm{s}$ ) and is determined by the application. Note that the physical CDR has to include the coding and protocol bits, so the information rate is usually less than the data 
rate. For instance a $3.125 \mathrm{Gbps} \mathrm{CDR}$ system with $8 \mathrm{~B} / 10 \mathrm{~B}$ coding might end up with an information rate of $3.125 \times 0.8=2.5 \mathrm{Gbps}$.

In the late 1990 s and through the better part of the first decade of 2000 , standards evolved to provide bit rate improvement in multiples of two or four per lane. This strategy quickly becomes unsustainable due to technology restrictions. It is, however, expedient to add an already functioning lane and double the throughput by using a multiplexer or to run four lanes at quarter of the speed by utilizing multiple sampling phases per bit. Intel has demonstrated a $10 \mathrm{Gbps} \times 47$ lane SerDes [69] for an 8-core Intel microprocessor. The bottleneck in this case becomes the multiplexer design and thus complexity can be managed more effectively for the given technology node. Although 40 Gbps CDR circuits have been reported, the challenges involved in their simulation, electrical design, package and thermal co-design and industrial testing are cost prohibitive [74]. The major trend is the improvement of throughput using independent lanes at frequencies that can be handled by the technology node expediently.

\subsubsection{Jitter generation}

Jitter generation (JGEN) in the transmitter circuitry is a major design concern at the TX end, since it only gets worse as the data progresses through the channel. It serves to reduce the timing margin of the CDR (and of the SerDes chain) as the data is recovered at the RX end. It can also get accentuated from VCO phase noise (PN) in the CDR due to the high-pass VCO PN characteristics, fluctuation of the charge pump outputs in PLLs and supply and substrate noise injection. It is measured in (fractions of) unit intervals (UI), usually mUI-pp. Jitter generation is cited as an aggregate value for the SerDes chain and the architecture of the CDR system or its particular contribution to this specification is not discussed in datasheets as a default strategy. 


\subsubsection{Jitter transfer}

Jitter Transfer (JTRAN) refers to the ability of the CDR circuit to suppress the incoming jitter at the specified frequency. The incoming data acquires jitter as it progresses through the channel. In data re-timing (repeater, SONET) applications, it is important that the jitter is not transferred (or even worse, amplified) from the input to the output. This incoming jitter should be suppressed within the constraints imposed by the CDR bandwidth. As the jitter amplitude dependent bandwidth is reached, attenuation of the jitter happens. Thus the critical portion where peaking happens has to be carefully designed. The JTRAN characteristics exhibit a typical low-pass profile and can be represented by Equation (3.2). A typical JTRAN curve is shown in Figure 3.40 for two arbitrary values of $K_{v c o}$.

$$
\frac{\phi_{\text {out }}}{\phi_{\text {in }}}(s)=\frac{1}{1+s / \omega_{-3 d B}} .
$$

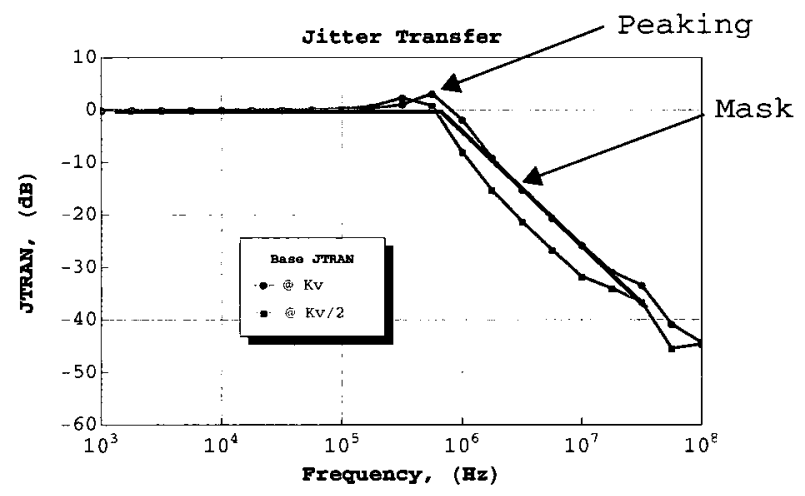

Figure 3.40 Typical jitter transfer characteristics

\subsubsection{Jitter tolerance}

Jitter Tolerance (JTOL) refers to the ability of the CDR circuit to tolerate the sinusoidal input jitter at a certain frequency for a certain BER. In general, the high frequency JTOL specifications are more difficult to meet than the low-frequency wander tracking 
specifications. This is because the PLL's ability to track is diminished beyond its loop bandwidth. Simulation of JTOL is discussed in Chapter 5.

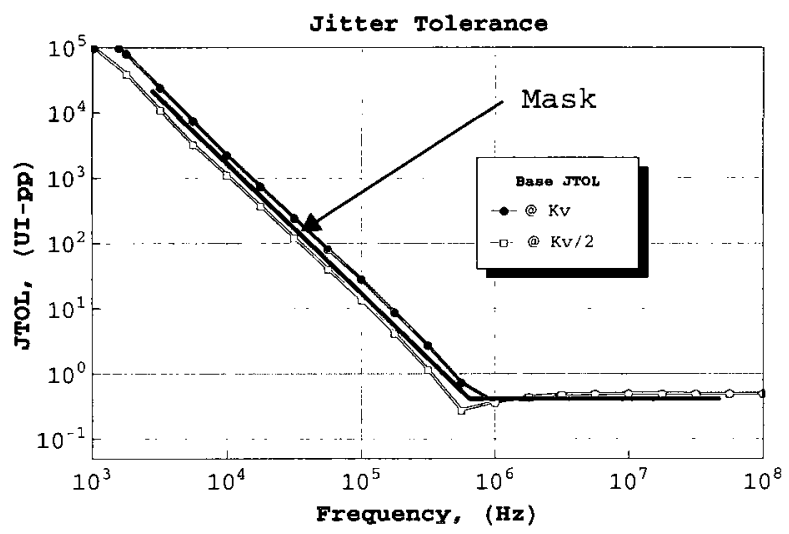

Figure 3.41 Typical jitter tolerance characteristics

\subsubsection{CID Tolerance}

For long data streams with no data transitions encountered in some applications, a lower limit on Consecutive Identical Digit (CID) count is also specified. The CDR circuit should be able to maintain lock and correctly recover the CID run followed by a PseudoRandom Bit Sequence (PRBS) of specified length. Digital CDR circuits can prevent the oscillator frequency from drifting significantly during CID run lengths and are of noticeable importance in this regard. For linear PLL-based architectures employing charge pumps, drifts in frequency occur as the loop-filter capacitors lose charge during a long CID run. This is referred to as the 'LF droop' and can be detected on an eye diagram. It disappears for a $101010 \ldots$ pattern and this serves as a debugging aid in analog CDR systems.

\subsubsection{Pattern dependence}

Deterministic Jitter (DJ) can be caused by certain repetitive data patterns. This deterministic error is observable only if the testing continues for several run lengths of the PRBS. Most notably, an alternating high-transition density pattern followed by a low-tran- 
sition density pattern would impart an asymmetric deterministic jitter to the data which should be successfully tolerated by the CDR circuit. Specialized sequences (e.g., K28.5) have been devised to facilitate the detection of pattern-related deterministic faults.

\subsection{Emergent themes in CDR design}

CDR circuit is an engineering product and as such has no single unifying theme. Components, algorithms, architectures and control methodologies cannot be satisfactorily used as pivots during a classification exercise. The designer has the unenviable task of deciding what combination of components, algorithms, architectures, design flow, clocking scheme, oscillator architectures and modeling strategy would deliver the intended results. This common requirement is perhaps a unified system-level analysis and an efficient simulation strategy that delivers the specified BER. A design flow that ensures firstpass silicon success is a topic of active research and of great commercial interest. For integrated CDR architectures it would not be biased, at this point, to list the dominant themes that have become evident as a result of the preceding review. These include, but are not limited to:

- CMOS over non-CMOS processes,

- Digital PDs over linear PDs,

- Reduced-rate designs vs. full-rate designs,

- DCO over VCO,

- Multi-phase clock generators over single phase oscillators,

- Digital filters, BIST and tuning over passive alternatives,

- Behavioral modeling over circuit-level simulations,

- Phase domain models over time domain models,

- Shared clock generators for multiple data lanes over higher speed individual lanes 
- Strategies for first silicon success and quick ATE testing vs. the expensive trialand-error approach,

- Encoded data vs. non-encoded data,

- PAM-2 over PAM-4 and other interesting signaling schemes,

- Digital design flow over an analog design flow and

- Discrete-time analysis over a continuous transfer function style analysis.

\subsection{Summary}

This chapter has reviewed, in some detail, the CDR components and architectures that have been presented in the open literature. A CDR circuit forms an integral part of the SerDes RX macro. It opens the horizontal eye by sampling the incoming data symbols at the centre to improve the BER. A DFE is required to open the vertical eye if the channel has already introduced excessive ISI and jitter prior to the CDR unit. As a first step, the CDR circuit either extracts the clock embedded in the data stream or uses a local PLLbased clock. Subsequently, the clock phase is adjusted to sample the data where the BER is the lowest.

Jitter was briefly reviewed as the major bottleneck afflicting CDR design and main specifications for a typical CDR system were listed. What is evident from the preceding discussion is that there needs to be a way to analyze and simulate CDR performance in an efficient way in order to evaluate various alternatives in the presence of jitter.

The underlying theme that may unite the linear, the bang-bang (or digital) and the oversampling architectures is elusive. The preceding review has not done much to elucidate any commonalities and such a unifying theme is not available in the literature. However, it revolves around the phase detection mechanism. Chapter 4 addresses this unification and is one of the major contributions of this dissertation. 


\section{Unified Phase Detector Modeling and Analysis}

"A new scientific truth does not triumph by convincing its opponents and making them see the light, but rather because its opponents eventually die, and a new generation grows up that is familiar with it."

Max Planck

\subsection{Chapter overview}

Phase detection is at the heart of CDR operation and CDR system classification. This chapter presents analytical equations for expected PD characteristics for bang-bang, oversampling and linear PD architectures in the presence of deterministic and random jitter. A common analysis theme unifies different PD architectures; and a simulation flow emerges for oversampling CDR system design as a result of this strategy. This unified PD analysis coupled with a practical simulation flow is a major contribution of this dissertation.

\subsection{Oversampling in serial links}

The idea of oversampling has been in the public domain for several decades. For Gbps data rates, a $3 \mathrm{XO}$ or a $5 \mathrm{XO}$ approach is considered practical. Up until now, no analytical framework exists in the literature to determine the minimum $O S R$ that must be employed for a particular type of input jitter distribution in order to achieve the required JTOL and JTRAN characteristics. This chapter presents such an analytical framework coupled with a behavioral-modeling-based simulation flow that can answer system-level CDR design questions. 


\subsection{The normal or Gaussian distribution}

This section defines essential terms used throughout the dissertation. Let $N(\mu, \sigma)$ be a one-dimensional normal distribution with a random variable (r.v.) $\phi$. The two parameters, the variance $\left(\sigma^{2}\right)$ and the mean $(\mu)$ define it completely as shown in Equation (4.1).

$$
f(\phi)=\frac{1}{\sqrt{2 \pi} \sigma} e^{-\frac{(\phi-\mu)^{2}}{2 \sigma^{2}}}=N(\mu, \sigma), \phi, \mu \in \Re, \sigma>0 .
$$

For $\mu=0, \sigma^{2}=1$, the distribution is known as a standard normal as given in Equation (4.2).

$$
N(0,1)=\frac{1}{\sqrt{2 \pi}} e^{-\frac{\phi^{2}}{2}} .
$$

The Gaussian distribution appears in all physical sciences and mathematics. The distribution has a maximum at $\mu$ and a decreasing slope thereafter. The word 'normal' signifies that the phenomenon being represented occurs naturally. Any deterministic activity manifests as a non-decreasing slope for a part of the distribution. The integral of the normal distribution is the error function (erf $(x))$ given in Equation (4.3).

$$
\int N(\mu, \sigma)=\frac{1}{2} \operatorname{erf}\left(\frac{\phi}{\sigma \sqrt{2}}\right) \text {. }
$$

If this integration is carried out over the range $(-\infty, \infty)$, then the total area under a normal distribution is found to be unity. The area under the standard normal distribution from a certain point $x$ to $\infty$ on the $x$-axis is labeled as $Q(x)$ and is defined in Equation (4.4). It also results in the observations given in Equation (4.5).

$$
\begin{gathered}
Q(x)=\int_{x}^{\infty} N(0,1) . \\
Q(-\infty)=1, Q(0)=0.5 \text { and } \mathrm{Q}(\infty)=0
\end{gathered}
$$


The functions $Q(x), \operatorname{erf}(x)$ and the complementary error function $(\operatorname{erfc}(x))$ are related for the standard normal case as shown in Equation (4.6).

$$
Q(x)=\frac{1}{2} \operatorname{erfc}\left(\frac{x}{\sqrt{2}}\right)=\frac{1}{2}\left[1-\operatorname{erf}\left(\frac{x}{\sqrt{2}}\right)\right] \text {. }
$$

The standard normal curve along with $Q(0.5)$ (shaded) is depicted in Figure 4.1 on linear and logarithmic scales. The parabolic relationship on the log scale in Figure 4.1(b) is obscured on the linear scale in Figure 4.1(a). It follows intuitively that the visual inspection of a linear scale histogram alone cannot deliver satisfactory BER readings and advanced automation is necessary for jitter instrumentation. While businesses and manufacturing concerns strive for a six-sigma $( \pm 3 \sigma)$ performance, jitter phenomena in HSSL require a 14-sigma $( \pm 7 \sigma)$ modeling that renders the human intuition as irrelevant.

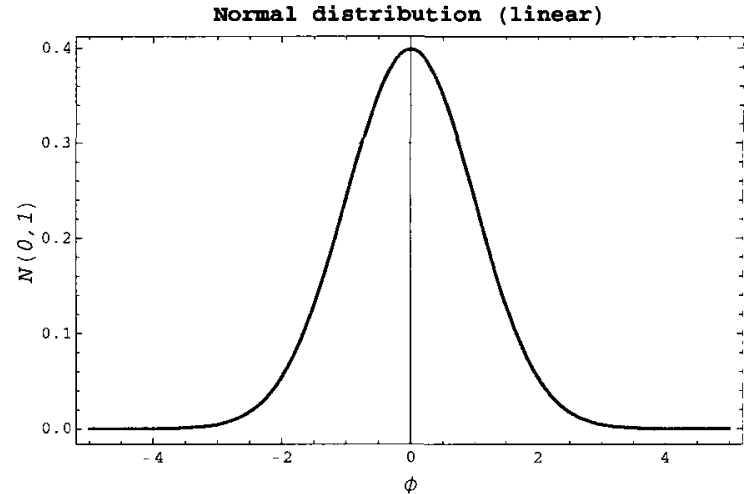

(a) Linear

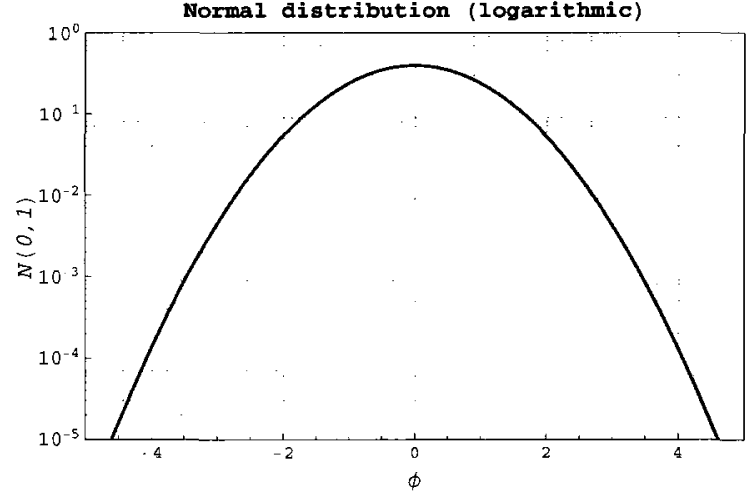

(b) Logarithmic

Figure 4.1 Standard normal distribution plots

The variable $\phi$ is used as the general r.v. associated with the normal distribution to represent phase error at $\mathrm{PD}$ input. The normal distribution represents a phenomenon that occurs mostly near the mean but naturally wanders in either direction in accordance with certain statistical metrics. In the context of the PD characteristics, the $x$-axis represents the phase error that can be either positive or negative but hovering around zero phase error in case of a phase-locked CDR circuit. The variable $\phi$ is not to be confused with the general description of phase as used in electrical engineering texts. Also the oversampling ratio 
$(O S R)$ neither derives from the ADC literature nor from the Sigma-Delta modulator literature. The $O S R$ refers to the distinct phases that can be called upon to detect the incoming data edge location within a UI.

\subsubsection{In the absence of the error function}

Some EDA software do not have precise representations of the $\operatorname{erf}(x)$ function in their repertoires. A simple tanh(.) substitution that minimizes the mean squared error over $-\infty<\phi<\infty$ between the two representations is given by Equation (4.7). The error reduces to less than $2 \%$ for $-2<\phi<2$ and rapidly diminishes thereafter.

$$
\operatorname{erf}(x)=\tanh (1.2028 x)
$$

The error and the absolute error between the two representations are plotted on linear and $\log$ scales, respectively, in Figure 4.2.

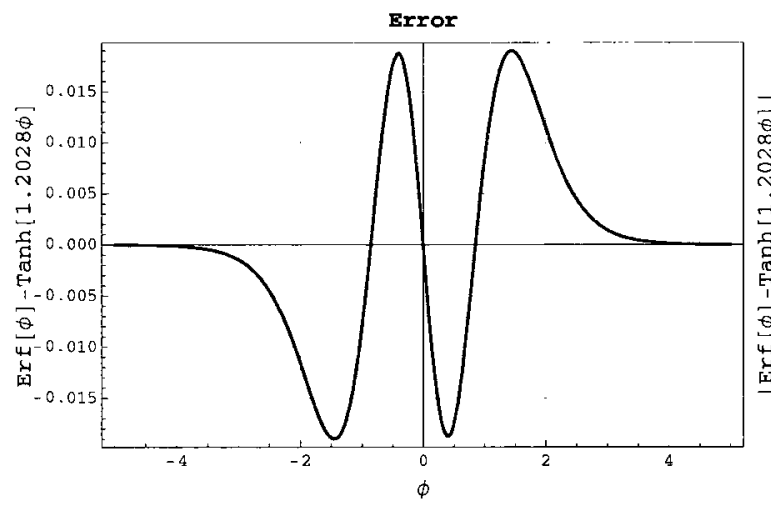

(a) linear scale

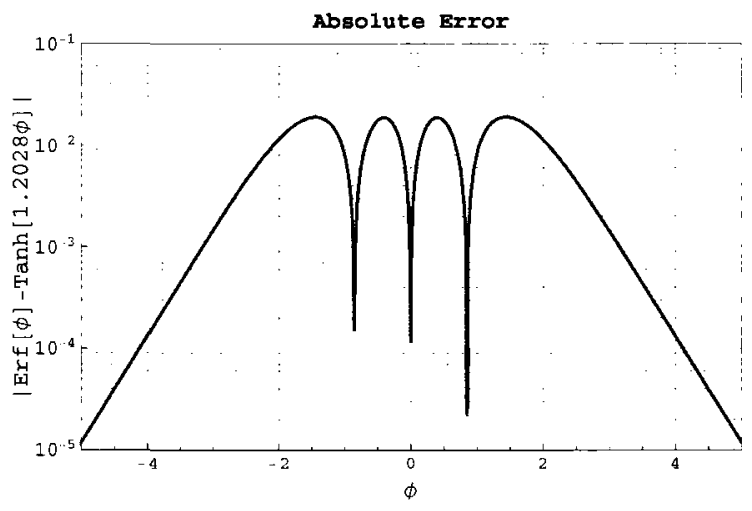

(b) log scale

Figure 4.2 The error between $\operatorname{erf}(x)$ and $\tanh (1.2028 x)$

\subsection{BBPD characteristics and the price of simplicity}

The BBPD transfer function in the phase domain is a signum function, i.e., it deciphers the sign of the input phase. If the clock edge is early compared to the data edge, the output is +1 , else it is -1 . This realization is profound, since the time-averaged BBPD characteristics are not determined exclusively by the BBPD's circuit design. The average 
BBPD output is governed by the distribution of the input edges. Individual data transitions are not considered for phase domain models but the average output of the PD in the presence of jitter can be found by convolving the transfer function of the BBPD with the input jitter distribution. For the case of Gaussian RJ, with a deviation of $\sigma$ and no asymmetry, edges fall equally on both sides of the peak in a locked state. Equation (4.8) is the result of convolving the normal distribution with the sgn(.) function.

$$
\operatorname{sgn}(\phi) * N(0, \sigma)=1-2 Q\left(\frac{\phi}{\sigma}\right)=\operatorname{erf}\left(\frac{\phi}{\sigma \sqrt{2}}\right)
$$

where $\operatorname{sgn}(\phi)$ is the signum function and '*' denotes the convolution operator. This methodology has been reported [33][34][35] for Gaussian jitter and the BBPD case only. The results showing various quantities are depicted in Figure 4.3.

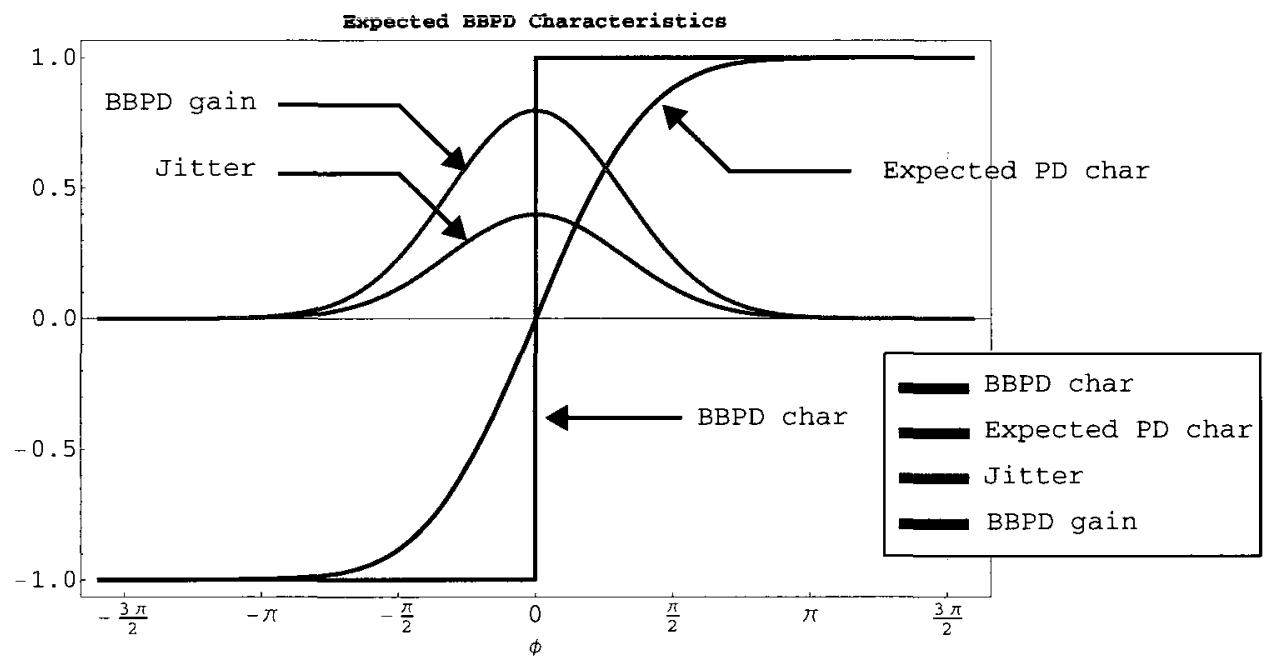

Figure 4.3 Expected BBPD characteristics

Linear PDs provide constant gain over much of the PD range making the analysis of PLL-FS systems mathematically convenient. General intuition would suggest that a similar constancy of PD gain would benefit CDR systems too, but this is not true.

For a CDR in lock, the slope at $\phi=0$ is the PD gain that determines the bandwidth of the CDR. Fluctuations at $\phi=0$ modulate the CDR bandwidth. A Gaussian style PD slope as observed in Figure 4.3 means that every incoming phase difference is treated 
differently depending on the phase error amplitude. The PD gain is not flat over the entire UI. Linear analyses involving BBPD cannot take this into account [32][34]. The PD gain is calculated as shown in Equation (4.9). A multiplier of 0.5 can account for the transition density of a truly random data pattern.

$$
\frac{d}{d \phi}\left[\operatorname{erf}\left(\frac{\phi}{\sigma \sqrt{2}}\right)\right]_{\phi \rightarrow 0}=\left.\frac{\sqrt{\frac{2}{\pi}}}{\sigma} e^{\frac{-\phi^{2}}{\sigma^{2}}}\right|_{\phi \rightarrow 0}=\frac{1}{\sigma} \cdot \sqrt{\frac{2}{\pi}}
$$

For a PD with a linear gain $K_{p d}$, the average output would not be affected by the input jitter. The non-intuitive result of a convolution of a constant, $c$, with a generalized normal distribution is the constant itself as shown in Equation (4.10).

$$
c^{*} N(\mu, \sigma)=c
$$

Commercial high speed CDR systems utilize a BBPD [32] and the Gaussian style PD gain actually performs a built-in filtering of the edges. If the edges fall near $\phi=0$, then the gain is almost flat. The edges that are further away are due to jitter and are suppressed. This scenario, however, does not hold if the jitter distribution becomes asymmetrical around $\phi=0$ or if the distribution changes. For most SerDes standards, DJ is part of the specification and asymmetry is the norm rather than the exception.

A linear gain over the entire PD range is not to be sought after all but it stabilizes CDR bandwidth if the PD gain is constant around $\phi=0$. This leads to many questions.

- Over what range, then, should a linear PD gain be sought in a CDR system? There is a dilemma since constant gain delivers constant bandwidth but it also means that jittered edges are not filtered degrading the HF JTOL.

- If a BBPD is not an ideal choice for asymmetric jitter, and a linear PD implementation is not feasible due to analog design challenges at high speeds, then how much oversampling should be employed?

- Does oversampling help stabilize CDR bandwidth? 
- Does oversampling help under asymmetric jitter conditions?

As the data rates approach $f_{\tau} / 5$ of the technology, BBPD circuit design becomes more cost-effective compared to a linear PD. If a flipflop can be implemented at a certain frequency, then a BBPD can also be implemented [32]. The price for this simplicity is the mathematical complexity, both for PLL-FS [62][63][64] and CDR systems [65][66] alike, if a BBPD is employed. The bit-by-bit behavior of a first-order BBPD, as explored in these publications is not helpful for CDR system design. With 10 billion such transitions happening per second for a $10 \mathrm{Gbps}$ link, individual events are not useful for understanding system behavior and are impossible to simulate practically [35]. A higher-level time-averaged view is required in the phase domain.

With data-dependent PD behavior, the reproducibility of BER results during testing is controlled by the applied input jitter and is not determined exclusively by the device under test (DUT). Testing of digital CDR circuits and that of SerDes systems employing them is, therefore, not straight-forward and often very frustrating.

As as result of this discussion, it is clear that a constant PD gain is helpful for utilizing a linear transfer function analysis that is well understood and mathematically expedient. For a better performing CDR system, a tapered PD gain is required to suppress RJ. For expedient system-level design, a mathematical framework is required that eliminates guesswork and parametrizes the PD characteristics given the type and quantity of input jitter. To improve CDR performance, this mathematical framework should be able to compare the time-averaged performances of the BBPD, the oversampling PDs and the linear PD such that a choice of optimum $O S R$ can be made. A closed-loop behavioral simulation flow based on such a unified PD analysis is presented in the remainder of this chapter.

\subsection{Transitioning from BBPD to oversampling PDs}

Figure 4.4(a) to Figure 4.4(d) depict a gradual transition from a BBPD to a linear PD with changing $O S R$. Any migration toward the linear PD characteristic is accompa- 
nied by movement on the $\phi$-axis (i.e., phase error) as well as the reduction of the jump along $y$-axis (i.e., output voltage or current). Figure 4.4(a) shows a 2XO PD or a BBPD.
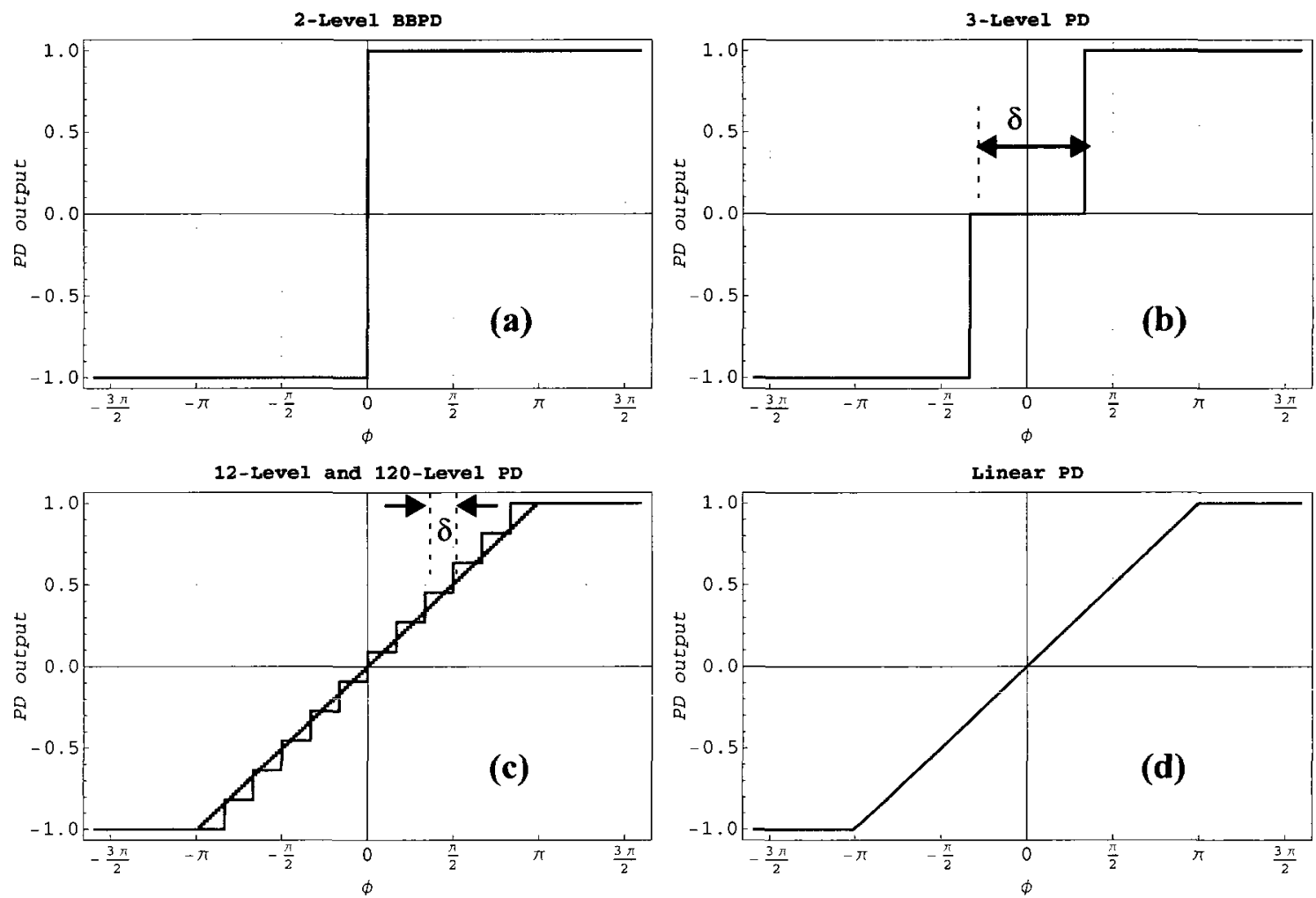

Figure 4.4 Transitioning from BBPD to linear

In Figure 4.4(b), 3XO PD characteristics are shown with a certain decision threshold in either direction. The maximum inter-phase spacing $(\delta)$ allowed for a NXO PD is limited to $2 \pi / O S R$. This generates equally-spaced phases. Here NXO PD means an oversampling PD with a general $O S R$. The 3 XO PD characteristics still look far from linear. Figure 4.4(c) shows the overlaid characteristics of a 12-XO PD and a 120-XO PD. Such systems are not practically feasible but the 120-XO PD characteristics look quite similar to the linear PD characteristics shown in Figure 4.4(d). This points to the possibility of achieving specified performance through a combination of $O S R$ and $\delta$ parameters given the power, area and jitter specifications for the CDR system. If the 120-XO PD characteristics resemble the linear PD characteristics visually, then it seems that mathematics would 
have no other choice but to behave accordingly. A proper understanding of oversampling trade-offs requires mathematical analysis that follows next.

\subsection{Generating phase detector transfer functions}

A $\operatorname{sgn}($.$) function can be written as a scaled unit step function with a biasing con-$ stant as shown in Equation (4.11). Let the unit step function be denoted by $\Theta$ and let $\Theta(0)=1 / 2$ so that,

$$
\Theta(\phi)=\frac{1}{2}(1+\operatorname{sgn}(\phi))
$$

In order to generate NXO PD characteristics, horizontally shifted and appropriately scaled $\Theta(\phi)$ functions can be added together in the phase domain. The convolution of these equations with parametrized jitter distribution functions gets computationally intensive for a larger $O S R$. Hence PD equations for $2<O S R<9$ have been generated for the purpose of this discussion. These characteristics have already been seen in Figure 4.4. Let $\Upsilon(O S R)$ denote the transfer function for the particular NXO PD with the inter-phase spacing $(\delta)$ and an oversampling ratio $(O S R)$ as shown in Equation (4.12).

$$
\left[\begin{array}{c}
\Upsilon(2) \\
\Upsilon(3) \\
\Upsilon(4) \\
\cdots \\
\Upsilon(9)
\end{array}\right]=\left[\begin{array}{c}
2 \Theta(\phi)-1 \\
\Theta\left(\phi-\frac{\delta}{2}\right)+\Theta\left(\phi+\frac{\delta}{2}\right)-1 \\
\frac{2}{3}[\Theta(\phi-\delta)+\Theta(\phi+\delta)+\Theta(\phi)]-1 \\
\ldots \\
\frac{1}{4}\left[\sum_{i=1}^{T, \text { odd }} \Theta\left(\phi-i \frac{\delta}{2}\right)+\sum_{i=1}^{7, \text { odd }} \Theta\left(\phi+i \frac{\delta}{2}\right)\right]-1
\end{array}\right],\{\sigma>0, \delta \geq 0\}
$$

The condition $\sigma>0$ ensures that the jitter distribution is not an impulse and the condition $\delta \geq 0$ allows for the BBPD in the limit. If $\delta=0$, all $\Upsilon(i)$ s reduce to the BBPD case, i.e., $\Upsilon(2)$ for $i \geq 2$. It is clear that $\Upsilon(2)$ represents the BBPD, $\Upsilon(3)$ represents the 
3XO PD and that $\Upsilon(\infty)$ represents a linear PD with infinite number of steps. Practically, the equivalence is reached when computation delivers non-significant advantage for the CPU time spent and the improvement in JTOL and JTRAN is no longer required to meet specifications. Each part in Equation (4.12) can now be convolved with an arbitrary jitter distribution, the generation of which is discussed next.

\subsection{Generating parametrized jitter distributions}

Figure 4.3 shows that the expected BBPD characteristics in the presence of symmetric RJ are centered on the mean of the jitter histogram. This means that half the edges are on either side of the mean. A locked CDR always works in this manner regardless of the jitter asymmetry [65] although the visualization becomes more involved.

Each of the $\Upsilon(i)$ equations has severai parts. There are two types of terms in Equation (4.12) — constant terms as well as $\Theta(\phi \pm c \delta)$ terms, where $c$ is a scalar constant and $\delta \geq 0$. The rules for convolution as shown in Equation (4.13) and Equation (4.14) have to be applied to each of the terms in the $\Upsilon(i)$ equations. This is true for the case of symmetric jitter in the $\phi$ domain as seen in Figure 4.3.

$$
\begin{gathered}
\Theta(\phi \pm c \delta) * N(\mu, \sigma)=\frac{1}{2}\left[1+\operatorname{erf}\left(\frac{\phi \pm c \delta}{\sqrt{2} \sigma}\right)\right] . \\
c * N(\mu, \sigma)=c \quad,\{\sigma>0, \delta \geq 0\} .
\end{gathered}
$$

\subsubsection{Asymmetric jitter distributions}

The asymmetry forces a non-decreasing condition over part of the jitter distribution. The asymmetric jitter distribution happens when DJ and RJ are both present. Equation (4.15) breaks the normal distribution in two parts each with a different jitter amplitude and separated by a distance of $2 k$, where $k$ is a positive scalar.

$$
f_{a s y}(\phi)=\operatorname{asy} N(\mu+k, \sigma)+(1-a s y) N(\mu-k, \sigma),
$$


where asy is the asymmetry factor, bounded by $0.25<a s y<0.75$. If the bound is chosen as $0<a s y<1$, then a pure RJ term occurs in either limit. This is the same condition as the case with no asymmetry as one of the Gaussian terms disappears. Various cases of symmetric and asymmetric jitter distribution are shown in Figure 4.5.
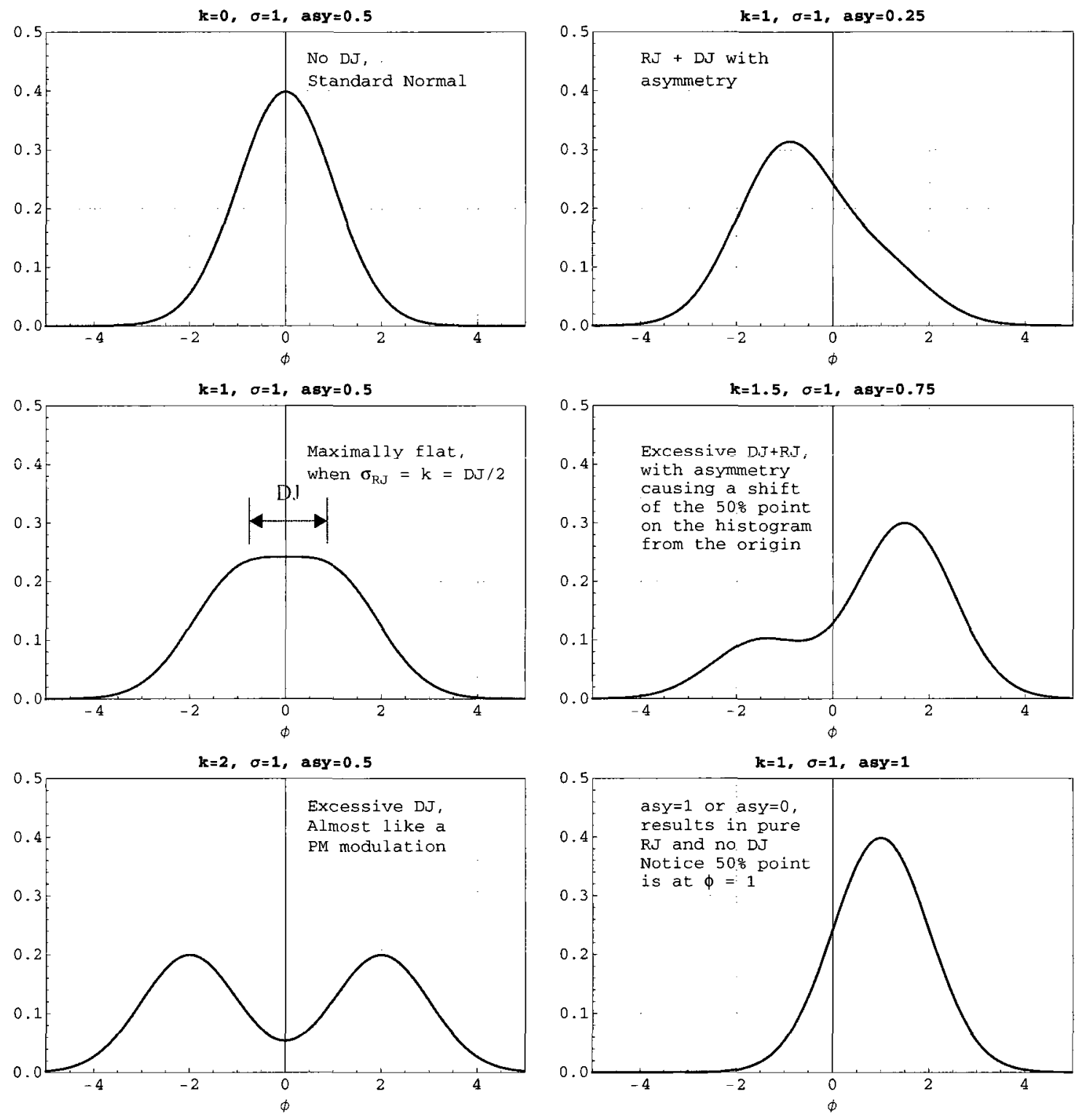

Figure 4.5 Jitter distributions with DJ and RJ 
Maximum flattening occurs at $\sigma=D J / 2$, after which the jitter distribution looks more like the case of PM modulation. The result of the convolution is slightly modified as shown in Equation (4.16).

$$
\begin{aligned}
& \Theta(\phi \pm x \delta) * f_{a s y}(\phi)=\frac{1}{2} a s y \cdot \operatorname{erfc}\left(\frac{k-\phi \mp x \delta}{\sqrt{2} \sigma}\right)+ \\
& \frac{1}{2}(a s y-1) \cdot\left[-2+\operatorname{erfc}\left(\frac{k+\phi \pm x \delta}{\sqrt{2} \sigma}\right)\right]
\end{aligned}
$$

The two Gaussian terms can have separate spreads and further generalization can be incorporated by introducing separate spreads $\left(\sigma_{1}\right.$ and $\left.\sigma_{2}\right)$, a scenario that is not pursued here. More complicated jitter profiles are rarely encountered but can be incorporated in this method. The total area under $f_{a s y}(\phi)$ remains unity. The parameter $\mu$ is usually set to zero to represent the sampling point such that $\phi<0$ and $\phi>0$ represent the previous and the current bit respectively. The histogram mean needs to be determined and the $\phi=0$ has to be shifted to accommodate the asymmetry. In order to highlight the asymmetry, the $\phi=0$ point has not been recalculated in Figure 4.5. However, it can be seen that the histogram mean does not coincide with the zero phase error if asymmetry is present. The expected characteristics of the NXO PD are centered at the histogram mean by virtue of $C D R$ operation; i.e, half the jittered edges on each side of the sampling point regardless of the distribution. The expected NXO PD equation thus derived after convolution are solved to find the zero crossing on the $\phi$ axis, and a shift is dynamically added depending on the selection of the parameters in the equation.

This asymmetry in the jitter hurts the conventional PLL-based CDR disproportionately since only one edge is being tracked. The other edge is used for sampling and a 50\% duty cycle is generally assumed. In the presence of asymmetric jitter or DCD, the BER performance of a conventional PLL CDR deteriorates and oversampling provides more robust performance [49][50]. 


\subsection{Expected characteristics for an arbitrary $\mathrm{NXO} P D$}

Now that the jitter components are in place and the symmetric distribution $f(\phi)$ is just a special case of the asymmetric distribution $f_{\text {asy }}(\phi)$ with asy $=0.5$, the characteristics of the NXO PDs can be convolved with the jitter distribution. This is done in Equation (4.17). Let $\tilde{\chi}(i, \phi)$ represent the expected characteristics of the NXO PD in question with $O S R$ set to $i$ such that,

$$
\tilde{\chi}(i, \phi)=\Upsilon(i) * f_{a s y}(\phi)
$$

For the case of no asymmetry, this characteristic would be passing through the $\phi=0$ point upon lock. Let $\chi(i, \phi)$ be the final expected PD characteristics, horizontally shifted to the histogram mean, due to the $f_{a s y}(\phi)$ distribution so that,

$$
\tilde{\chi}(i, \phi)=\left.\chi(i, \phi)\right|_{\text {asy }=0.5}
$$

The partial Mathematica ${ }^{\circledR}$ list of $\tilde{\chi}(i, \phi)$ vector is shown in Figure 4.6 in bitmap form and would span many pages of typed equations.

$$
\begin{aligned}
& \left\{-\operatorname{asy} \operatorname{Erf}\left[\frac{k-\phi}{\sqrt{2} \sigma}\right]-(-1+\operatorname{ass}) \operatorname{Err}\left[\frac{k+\phi}{\sqrt{2} \sigma}\right\} \frac{1}{2}\left((-1+\operatorname{asy}) \operatorname{Erf}\left[\frac{\delta-2(k+\phi)}{2 \sqrt{2} \sigma}\right]-(-1+\operatorname{asy}) \operatorname{Erf}\left[\frac{\delta+2(k+\phi)}{2 \sqrt{2} \sigma}\right]+\operatorname{asy}\left(-2+\operatorname{Erc}\left[\frac{2 k-\delta-2 \phi}{2 \sqrt{2} \sigma}\right]+\operatorname{Erc}\left[\frac{k+\frac{\delta}{2}-\phi}{\sqrt{2} \sigma}\right]\right)\right)\right. \\
& \frac{1}{3}\left(\operatorname{Erf}\left[\frac{k+\phi}{\sqrt{2} \sigma}\right]+\operatorname{Ext}\left[\frac{k-\delta+\phi}{\sqrt{2} \sigma}\right]+\operatorname{Erf}\left[\frac{k+\delta+\phi}{\sqrt{2} \sigma}\right]-\operatorname{ary}\left[\operatorname{Err}\left[\frac{k-\phi}{\sqrt{2} \sigma}\right]+\operatorname{Er}\left[\frac{k-\sigma-\phi}{\sqrt{2} \sigma}\right]+\operatorname{Er}\left[\frac{k+\delta-\phi}{\sqrt{2} \sigma}\right]+\operatorname{Er}\left[\frac{k+\phi}{\sqrt{2} \sigma}\right]+\operatorname{Ern}\left[\frac{k-\delta+\phi}{\sqrt{2} \sigma}\right]+\operatorname{Er}\left[\frac{k+\delta+\phi}{\sqrt{2} \sigma}\right]\right)\right)
\end{aligned}
$$

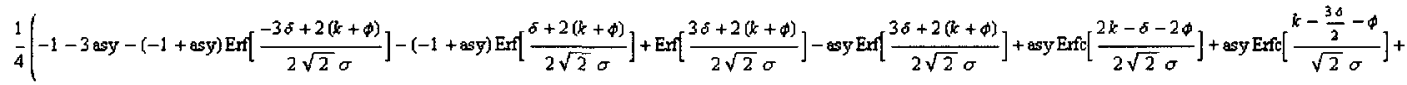

$$
\begin{aligned}
& \left.\operatorname{asy} \operatorname{Erc}\left[\frac{k+\frac{\delta}{2}-\phi}{\sqrt{2} \sigma}\right]+\operatorname{asy} \operatorname{Erfc}\left[\frac{k+\frac{3 \delta}{2}-\phi}{\sqrt{2} \sigma}\right]-(-1+\operatorname{ssy}) \operatorname{Erf}\left[\frac{\delta-2(x+\phi)}{2 \sqrt{2} \sigma}\right]\right), \frac{1}{s}\left[\operatorname{Erf}\left[\frac{k+\phi}{\sqrt{2} \sigma}\right]+\operatorname{Erf}\left[\frac{k-2 \delta+\phi}{\sqrt{2} \sigma}\right]+\operatorname{Err}\left[\frac{k-\sigma+\phi}{\sqrt{2} \sigma}\right]+\operatorname{Er}\left[\frac{k+\delta+\phi}{\sqrt{2} \sigma}\right]+\operatorname{Erf}\left[\frac{k+2 \delta+\phi}{\sqrt{2} \sigma}\right]-\right. \\
& \operatorname{ary}\left[\operatorname{Erf}\left[\frac{k-\phi}{\sqrt{2} \sigma}\right]+\operatorname{Err}\left[\frac{k-2 \delta-\phi}{\sqrt{2} \sigma}\right]+\operatorname{Er}\left[\frac{k-\delta-\phi}{\sqrt{2} \sigma}\right]+\operatorname{Er}\left[\frac{k+\delta-\phi}{\sqrt{2} \sigma}\right]+\operatorname{Err}\left[\frac{k+2 \delta-\phi}{\sqrt{2} \sigma}\right]+\operatorname{Er}\left[\frac{k+\phi}{\sqrt{2} \sigma}\right]+\operatorname{Er}\left[\frac{k-2 \delta+\phi}{\sqrt{2} \sigma}\right]+\operatorname{Er}\left[\frac{k-\delta+\phi}{\sqrt{2} \sigma}\right]+\operatorname{Erf}\left[\frac{k+\delta+\phi}{\sqrt{2} \sigma}\right]+\operatorname{Erf}\left[\frac{k+2 \delta+\phi}{\sqrt{2} \sigma}\right]\right), \\
& \frac{1}{6}\left(-1-5 \operatorname{asy}-(-1+\operatorname{asy}) \operatorname{Err}\left[\frac{-5 \delta+2(x+\phi)}{2 \sqrt{2} \sigma}\right]-(-1+\operatorname{ssy}) \operatorname{Erf}\left[\frac{-3 \delta+2(x+\phi)}{2 \sqrt{2} \sigma}\right]+\operatorname{Er}\left[\frac{\delta+2(x+\phi)}{2 \sqrt{2} \sigma}\right]+\operatorname{Erf}\left[\frac{3 \delta+2(x+\phi)}{2 \sqrt{2} \sigma}\right]+\operatorname{Erf}\left[\frac{5 \delta+2(x+\phi)}{2 \sqrt{2} \sigma}\right]+\right. \\
& \operatorname{Erc}\left[\frac{\delta-2(k+\phi)}{2 \sqrt{2} \sigma}\right]+\arg \left[-4+\operatorname{Erf}\left[\frac{\delta-2(k+\phi)}{2 \sqrt{2} \sigma}\right]+\operatorname{Erc}\left[\frac{2 k-\delta-2 \phi}{2 \sqrt{2} \sigma}\right]+\operatorname{Erf}\left[\frac{k-\frac{5 \delta}{2}-\phi}{\sqrt{2} \sigma}\right]+\operatorname{Ercc}\left[\frac{k-\frac{30}{2}-\phi}{\sqrt{2} \sigma}\right]+\right. \\
& \left.\operatorname{Err}\left[\frac{k+\frac{\phi}{2}-\phi}{\sqrt{2} \sigma}\right]+\operatorname{Err}\left[\frac{t+\frac{3 \Delta}{2}-\phi}{\sqrt{2} \sigma}\right]+\operatorname{Erc}\left[\frac{k+\frac{5 \Delta}{2}-\phi}{\sqrt{2} \sigma}\right]+\operatorname{Err}\left[\frac{\delta+2(k+\phi)}{2 \sqrt{2} \sigma}\right]+\operatorname{Erc}\left[\frac{3 \sigma+2(k+\phi)}{2 \sqrt{2} \sigma}\right]+\operatorname{Erc}\left[\frac{5 \sigma+2(k+\phi)}{2 \sqrt{2} \sigma}\right]\right), \\
& \frac{1}{7}\left[\operatorname{Er}\left\{\frac{k+\phi}{\sqrt{2} \sigma}\right]+\operatorname{Er}\left\{\frac{k-3 \delta+\phi}{\sqrt{2} \sigma}\right]+\operatorname{Er}\left\{\frac{k-2 \delta+\phi}{\sqrt{2} \sigma}\right]+\operatorname{Erf}\left[\frac{k-\delta+\phi}{\sqrt{2} \sigma}\right]+\operatorname{Erf}\left[\frac{k+\delta+\phi}{\sqrt{2} \sigma}\right]+\operatorname{Ert}\left[\frac{k+2 \delta+\phi}{\sqrt{2} \sigma}\right]+\operatorname{Err}\left[\frac{k+3 \delta+\phi}{\sqrt{2} \sigma}\right]-\right.
\end{aligned}
$$

Figure 4.6 Generalized NXO PD equations 
In the more general case, $\tilde{\chi}(i, \phi)$ needs to be solved for the zero crossing. This solution is unique since the histogram mean is always unique. Let $\phi_{c}$ be the point where the expected crossing happens, then the general case is shown in Equation (4.19).

$$
\chi(i, \phi)=\tilde{\chi}\left(i, \phi+\phi_{c}\right)
$$

where $\phi_{c}$ is the solution of $\tilde{\chi}(i, \phi)=0$ for the selected parameters. Equation (4.19) reduces to Equation (4.18) when $\phi_{c}=0$ at $a s y=0.5$. For high OSR values the real-time interactivity can get sluggish on slower CPUs. For practical $O S R \mathrm{~s}$, this dynamic computation does not pose any issue for a typical computer circa 2010.

The gain of the expected NXO PD characteristics can be found by differentiating Equation (4.19) w.r.t. $\phi$, as shown in Equation (4.20).

$$
\Psi(i, \phi)=\left[\left.\frac{d}{d \phi}[\tilde{\chi}(i, \phi)]\right|_{\phi \rightarrow \phi+\phi_{c}}\right]
$$

The vector of computed equations for $\Psi(i, \phi)$ does not promote any human understanding and is, therefore, not listed here. Generation and visualization of such equations requires a mathematics software with an advanced symbolic capability and a fast CPU.

The RJ and DJ parameters in these equations are given by the channel conditions. The asymmetry might be acquired due to the channel, the DFE, supply, substrate disturbance or it could also be pattern or design related.

Two parameters relate to the PD itself. The $O S R$ is selected based on the area, power and design complexity. The inter-phase spacing $(\delta)$ is determined by the number of oscillator phases. Due to the number of free parameters, it is not feasible to plot sets of 2D curves since for $n$ parameters it would require $2^{n-2}$ 2D-plots and contribute little to the understanding. An interactive software proves to be more useful such that a broad understanding of the design process can be facilitated for the choice of input jitter parameters. 
The next step is to extract and convert the equations in order to transport them to an AMS style behavioral simulator to perform accurate but efficient system-level CDR simulations.

\subsection{Expected characteristics of a linear PD}

This section appears in its current location since it is easier to discuss the concept of expected PD characteristics at this point. Since the gain of the ideal linear sawtooth PD is not dependent on external parameters, it retains its shape in the presence of various jitter distributions. Linear detection suffers from practical circuit design problems as discussed elsewhere in the dissertation. The range for phase detection is $(-\pi, \pi)$ and a FD mechanism takes over thereafter. The sawtooth linear PD characteristics have discontinuities at $\pm \pi$ and unless a constant value is used for PD slope using intuition, the math involving integrals, differentials and Gaussian non-linearities runs into frequent difficulties as shown in Figure 4.7. It is ironic that the BBPD and a linear PD would share something such as a pure mathematical discontinuity. Linear PDs remain a good vehicle for understanding and for transfer function style analysis.

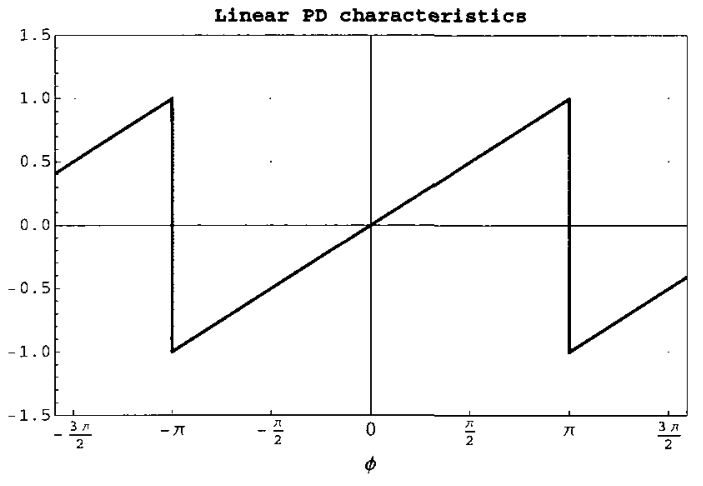

(a) Linear PD characteristics

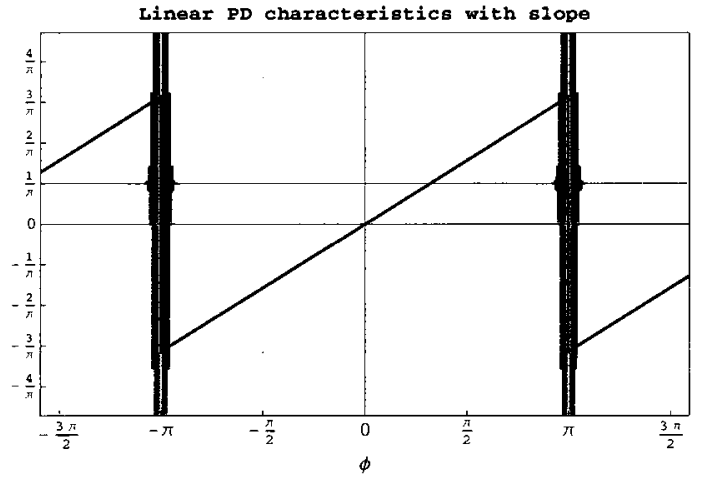

(b) Slope discontinuity

Figure 4.7 Linear PD and the discontinuity

\subsection{Expected characteristics of a clip PD}

Linear or exponential, characteristics of real devices saturate soon (or sooner than desired). Let us define a clip PD, whose characteristics saturate at \pm 1 for $\phi= \pm \pi$. This 
saturation represents the transition to a FD mechanism. The method of the preceding sections is applied and the long-winded equations are computed for the expected characteristics. The clip PD is not burdened by the effects of oversampling and phase spacing; it does not have a discontinuity either and can serve as a perfect analytical and theoretical device. It is intuitively expected that the clip PD characteristics would be modified starting near the edges of the clip PD range due to RJ, DJ and asymmetry. This also serves as a test case for comparison with the general NXO PD that depends on $O S R$ and $\delta$ for its operation. Figure 4.8(a) shows the clip PD characteristics and the ideal slope in the presence of no jitter. Figure 4.8(b) shows the same plot, with many more curves having appeared due to RJ with $\sigma=1.0 \mathrm{rad}$. The plots use the same legend as Figure 4.3, with the addition of a golden trace that represents the ideal PD gain.

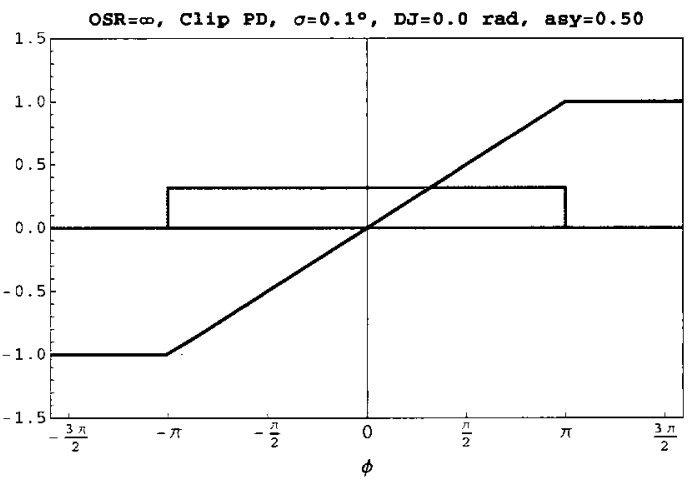

(a) PD Characteristics and slope

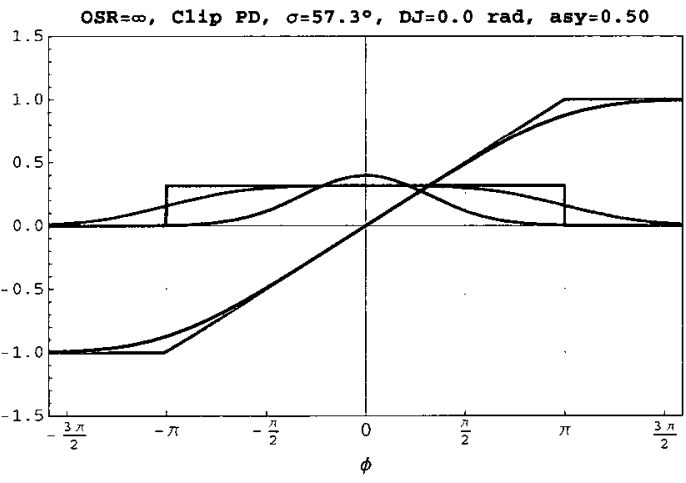

(b) Expected characteristics

Figure 4.8 Clip PD as an analytical device

The differences between ideal and expected PD characteristics can be clearly seen in Figure 4.8(b). The BBPD $(\Upsilon(2))$ and the clip PD $(\Upsilon(\infty))$ realizations could not be further apart but share one attribute. Neither is dependent on the phase spacing parameter $\delta$. The advantages that accrue with oversampling are critically dependent on the inter-phase spacing since with $\delta=0$, any NXO PD reduces to a BBPD.

The reason for the choice of the label 'clip PD' is because there is a command in Mathematica $^{\circledR}$ that behaves similarly (see "Clip[x]" [160]). 


\subsection{Interactive software}

Mathematica $^{\circledR}$ is a commercially available software with support for symbolic mathematics and dynamic interactivity and is chosen for this work. All pertinent information about the software can be found at the official website [160]. In summary:

- The generalized equations for NXO PDs are formulated (Section 4.6)

- The parametrized equations for the jitter are generated (Section 4.7)

- The two items above are convolved, collected and archived for faster real-time execution (Section 4.8)

- The expected characteristics are shifted to account for jitter asymmetry as the parameters are changed interactively in real-time (Equation (4.19)).

- The PD gain is calculated using differentiation of the expected PD characteristics while holding all other parameters constant (Equation (4.20))

- The PD transfer function, NXO PD characteristics, PD gain and the jitter distribution are updated interactively.

Various results and screenshots are presented in the following sections to promote an understanding of the trade-offs. The value of this process is that once the $O S R$ and $\delta$ have been chosen for the given jitter, the equations are available and can be transported to the behavioral simulator. The availability of time-averaged NXO PD equations is an advantage over prior art and is a major contribution of this dissertation.

\subsubsection{Plotting software output}

The output of interactive software can be saved to a graphics format file. While output on static paper cannot capture the essence of interactivity, the resulting set of plots are explained to highlight the important features. The plots in this section in particularand in this chapter in general-do not contain individual legends to avoid clutter. Instead the legend from Figure 4.3 is adopted. Each plot is individually labeled at the top for parameters. The plots proceed from left to right and top to bottom unless otherwise speci- 
fied. The shaded portion represents $50 \%$ area under the jitter histogram starting at $-\infty$; it becomes important for the asymmetric jitter case. The last picture in all sets of results in this section is the comparison with the clip PD performance for the same jitter parameters.

Plots appear on a separate page for each test case following the explanations that appear in

Section 4.12. The case with $O S R=9$, although generated and captured, is omitted so as to have a rectangular array of results fit on a page; besides it does not provide additional understanding.

\subsection{Test cases}

The representative test cases are listed in Table 4.1 and the plotted outputs appear from Figure 4.9 through to Figure 4.15.

TABLE 4.1: Test Cases - PD performance evaluation

\begin{tabular}{|c|c|c|c|c|c|c|}
\hline Case & OSR & asy & $\mathbf{D J}=\mathbf{2} \mathbf{k}$ & $\mathbf{R J}(\sigma)$ & $\begin{array}{c}\text { Interphase } \\
\text { spacing }(\delta)\end{array}$ & Remarks \\
\hline 1 & $2-8$ & 0.5 & $\mathrm{k}=0$ & $\mathrm{UI} / 14$ & $2 \pi / O S R$ & $\begin{array}{l}\text { No asymmetry, RJ only, } \sigma \text { for } 10^{-12} \\
\text { performance, no DJ }\end{array}$ \\
\hline 2 & $2-8$ & 0.5 & $\mathrm{k}=0.25$ & $\mathrm{UI} / 14$ & $2 \pi / O S R$ & Same as case 1 but with the onset of DJ \\
\hline 3 & $2-8$ & 0.5 & $\mathrm{k}=0.50$ & $\mathrm{UI} / 14$ & $2 \pi / O S R$ & Same as case 1 but with flat DJ \\
\hline 4 & $2-8$ & 0.5 & $\mathrm{k}=1.5$ & $\mathrm{UI} / 14$ & $2 \pi / O S R$ & DJ resembles phase modulation \\
\hline 5 & $2-8$ & 0.5 & $\mathrm{k}=1$ & $\mathrm{UI} / 9$ & $2 \pi / O S R$ & Excessive $\mathrm{RJ}$ and $\mathrm{DJ}$ \\
\hline 6 & $2-8$ & 0.25 & $k=1$ & $\mathrm{UI} / 9$ & $2 \pi / O S R$ & Excessive DJ, RJ with asymmetry \\
\hline 7 & 8 & 0.5 & $\mathbf{k}=1$ & $\mathrm{UI} / 9$ & sweep & $\begin{array}{l}\text { Same as case } 6 \text {, showing the effect of } \delta \\
\text { on characteristics for OSR }=8 \text { every } 15^{\circ} \\
\text { upto a maximum of } 45^{\circ}\end{array}$ \\
\hline 8 & 3 & 0.5 & $\mathbf{k}=1$ & $\mathrm{UI} / 9$ & sweep & $\begin{array}{l}\text { Same as case } 7 \text {, showing the effect of } \delta \\
\text { on characteristics for OSR }=3 \text { every } 40^{\circ} \\
\text { upto a maximum of } 120^{\circ}\end{array}$ \\
\hline
\end{tabular}




\subsubsection{Case 1}

Case 1 is the application of RJ only with no DJ or asymmetry. The plots are shown in Figure 4.9. The shaded area on the jitter distribution is divided equally on both sides of $\phi=0$. The gain becomes sufficiently linear for $O S R=6$. The $\sigma$ for $\mathrm{RJ}$ is set to $25.7^{\circ}$ as $7 \sigma$ results in a $0.5 \mathrm{UI}$ spread. This case is intended as a demonstration since DJ is always present.

\subsubsection{Case 2}

Case 2 is the same as Case 1 but with the initial onset of DJ. The plots are shown in Figure 4.10. It can be seen that a BBPD with $O S R=2$ performs with a high gain and fairly linear characteristics for a $\pm \sigma$ range. Without the benefit of an interactive software, this might lead to a conclusion that a BBPD might be a good choice. However the introduction of asymmetry frequently provides evidence to the contrary. The reader can notice the improvement in the $3 \mathrm{XO}$ and the $5 \mathrm{XO} \mathrm{PD}$ case. The flattening of the jitter distribution provides a linear gain to the PD near $\phi=0$ and thus characteristics are improved.

\subsubsection{Case 3}

Case 3 introduces more DJ such that flatness is visible on the jitter histogram. The plots are shown in Figure 4.11. The onset of a deadzone in the BBPD case is not visible yet. For various values of RJ and DJ, a PD with a higher $O S R$ performs better in the event of asymmetry. The question is not whether the 5XO PD is better than a 3XO PD. The idea is to search for the least valued $O S R$ solution to handle the jitter present at the input. The reader can notice the almost linearized $O S R=3$ case. Hence the addition of DJ creates problems for the BBPD but is handled better by PDs with a higher $O S R$. 


\subsubsection{Case 4}

Case 4 represents excessive modulation style DJ. This is depicted in Figure 4.12. Such DJ is added in a JTOL simulation and this case is included for demonstration only. The analytical difficulty involved in convolving a sinusoidal modulation histogram with discontinuities is significant and prevents a simple computation of the results. In effect, since the modulation is deterministic, and edges move to either side of the locking point such that the density of the edges at the locking point is reduced. The reader can notice the pronounced deadzone for the case of the BBPD. For $O S R=5$ or above, the situation does not improve significantly leading up to the clip PD and hence there is no point in increasing the $O S R$. As a frequency detection mechanism, BBPD performs poorly but higher $O S R$ values perform better since the edge visibility during the UI is improved. For PM modulation, the integral path in called upon to assist the NXO PD inside the CDR system.

\subsubsection{Case 5}

Case 5 addresses the case of excessive RJ and DJ. The plots appear in Figure 4.13. Once again, the non-intuitive result for the BBPD is evident. Increasing the RJ has reduced the deadzone for the BBPD. In general, the linearity of the gain does not change much after $O S R=5$. The next test case introduces asymmetry.

\subsubsection{Case 6}

Case 6 is the same as Case 5 but with severe asymmetry added. The results are shown in Figure 4.14. For Case 5, the BBPD performance did improve with addition of $\mathrm{RJ}$, the introduction of asymmetry produces a very different picture. The asymmetry can sway the histogram from side to side during CDR operation. The JTOL and JTRAN for the CDR under asymmetry is determined by the worst portion of the characteristics on either side of $\phi=0$. This is where the advantages of oversampling become obvious. It 
can be noticed that the PD performance for $O S R>4$ is vastly improved for this especially degenerate case of excessive RJ and DJ. Interactively, it can be seen that changing the asymmetry from side to side does not change the gain significantly, thus resulting in a robust CDR design over PVT and design-induced variations. The reader can notice the shaded portion on the histogram and compare with previous cases.

\subsubsection{Case 7 and Case 8}

For Figure 4.15, the results in the first column $(O S R=8)$ and the second column $(O S R=3)$ are to be read from top to bottom. The effect of varying $\delta$ on PD performance has been recorded. It can be seen that the $O S R=3$ and $O S R=8$ result in fairly similar looking characteristics and even achieve optimum performance at different values of $\delta$. The case of equally spaced phases is more desirable since it gives edge visibility over the entire UI and thus provides more information and more gain from the PD. Practically, there can be many variations of this theme. One could easily choose 3 out of 8 phases, in which case the phase spacing is fixed by the higher $O S R$ value. The advantage in doing so is that a different phase spacing can be chosen for acquisition and locked modes. The $O S R$ of the PD is determined by the number of phases being used in the PD and not upon the number of phase available from the multi-phase oscillator. The phase step at the output that occurs during CDR operation is not as abrupt if many phases are available to choose from. Practically, this is limited by the power required in the multiphase oscillator circuit. 

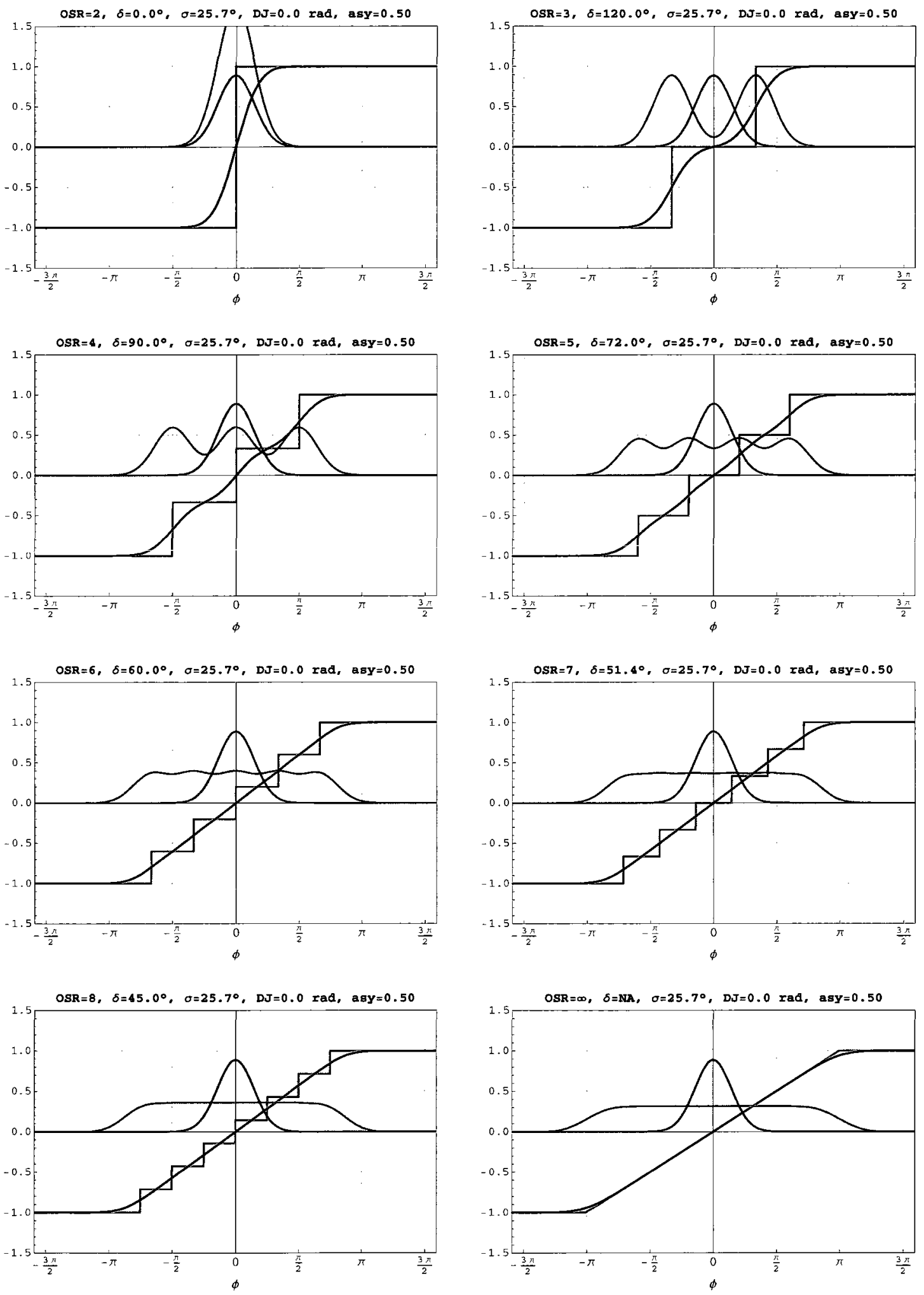

Figure 4.9 Case 1 - RJ only, maximal phase spacing 

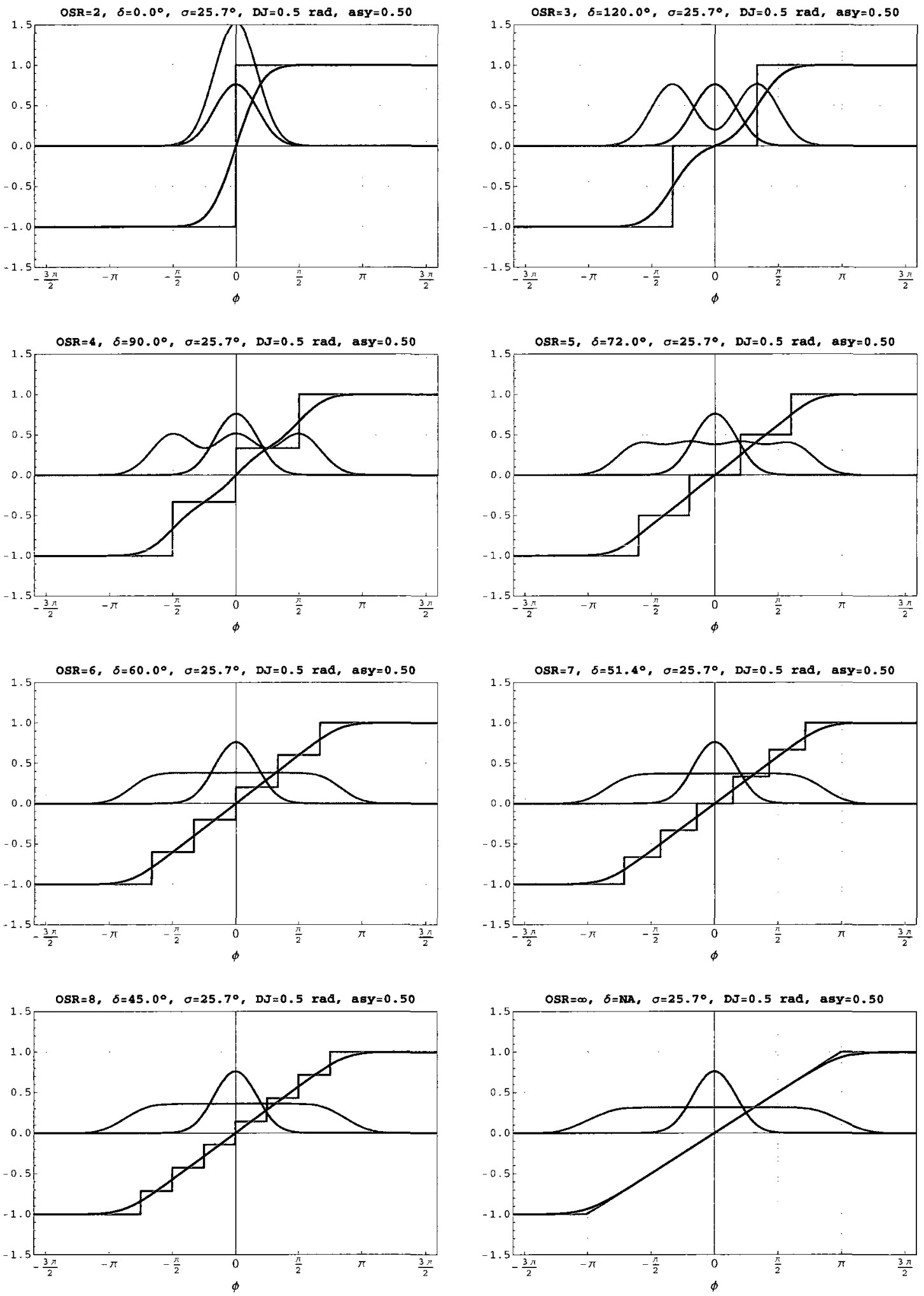

Figure 4.10 Case 2 - RJ + DJ, maximal phase spacing 

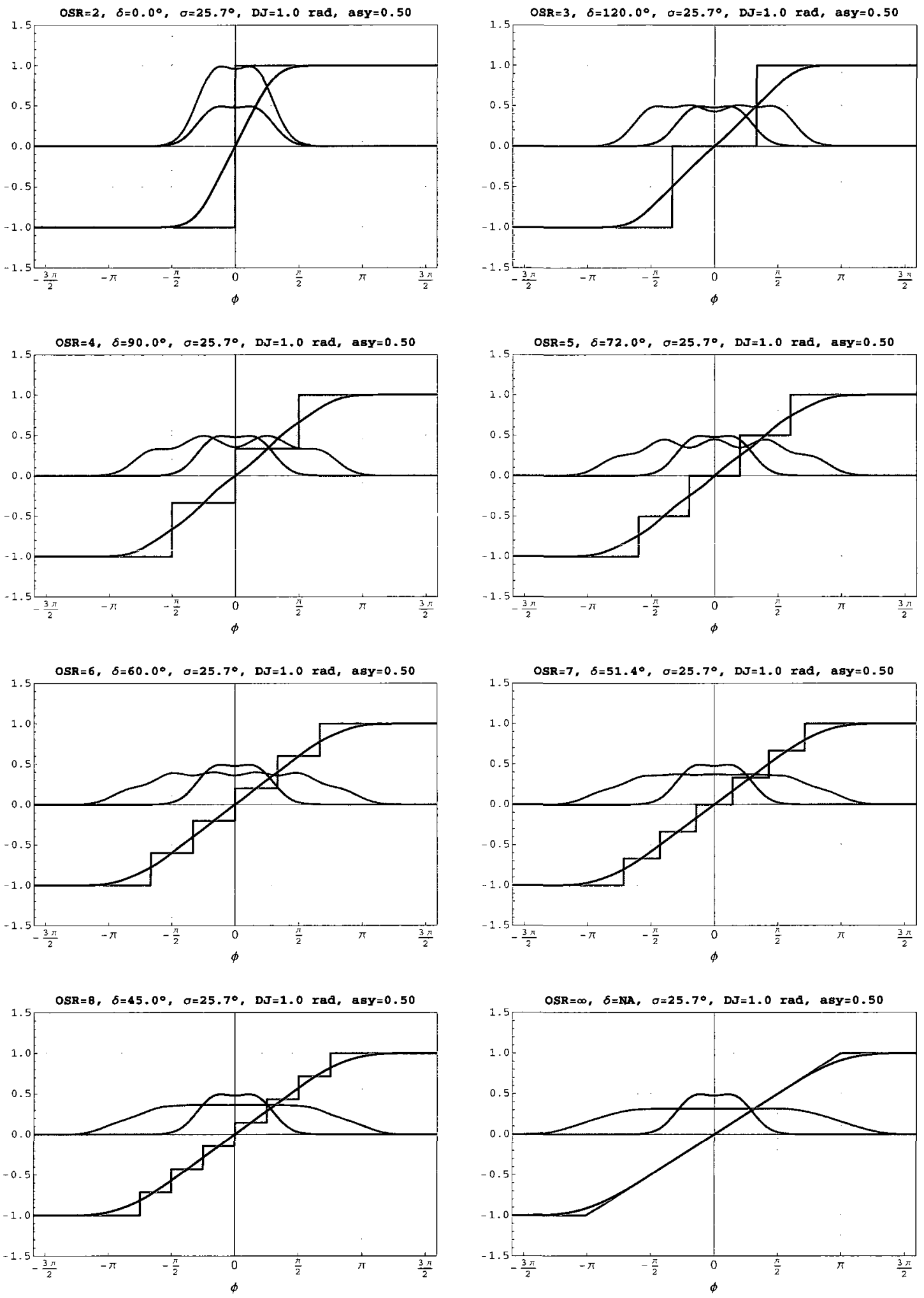

Figure 4.11 Case 3 - Enough DJ to cause histogram flattening 

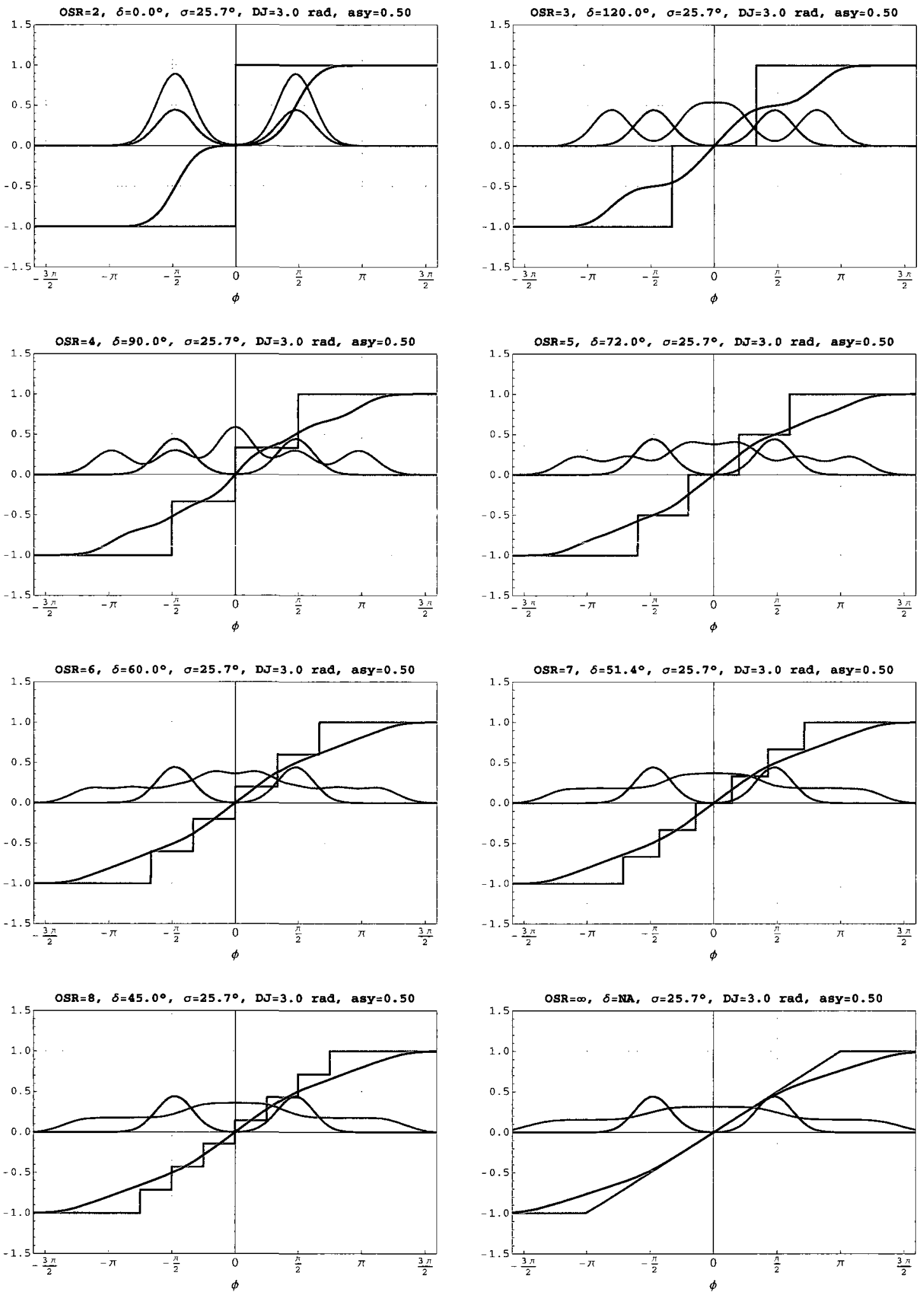

Figure 4.12 Case 4 - PM modulation style DJ 

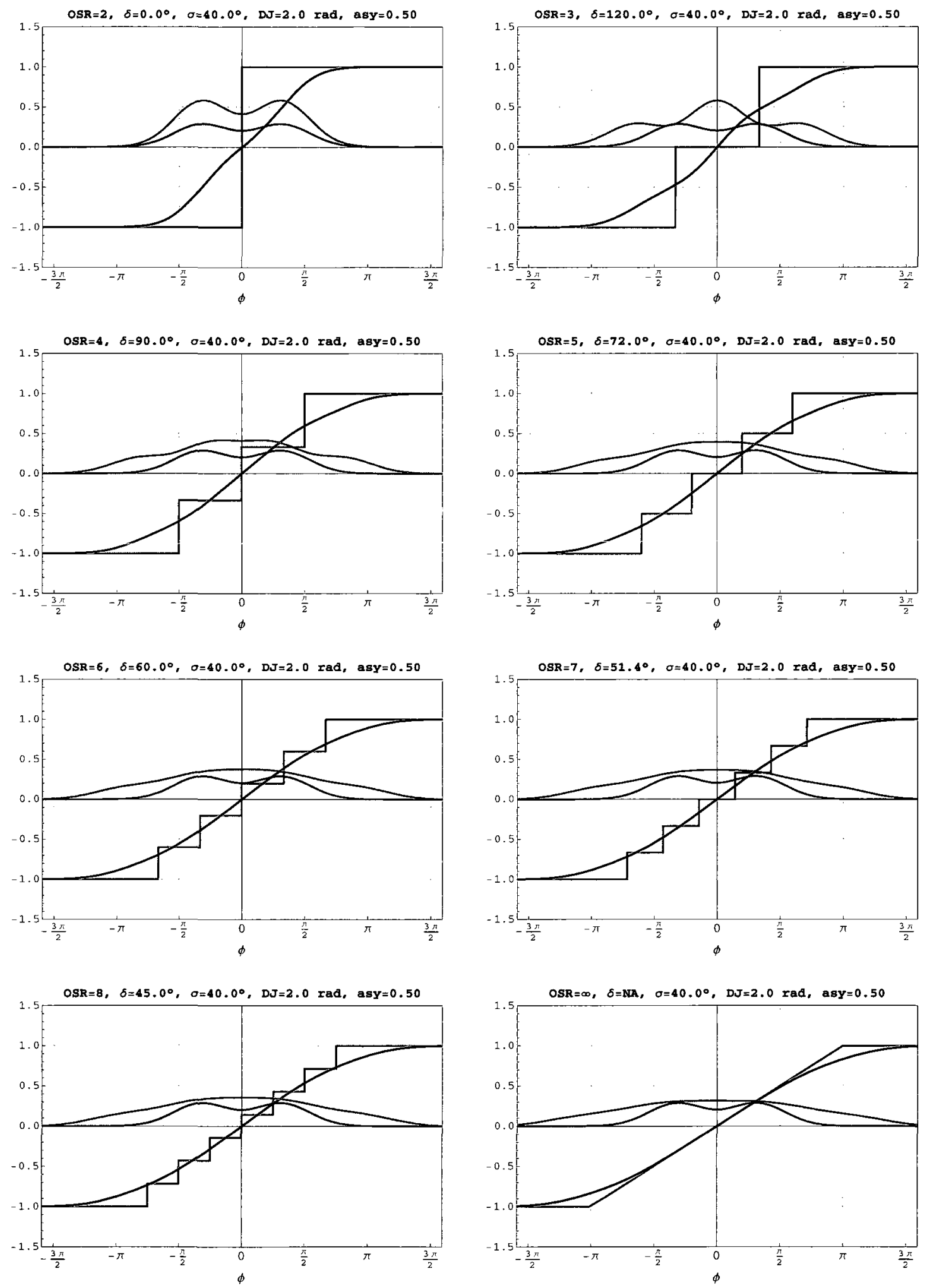

Figure 4.13 Case 5 - Excessive RJ + DJ 

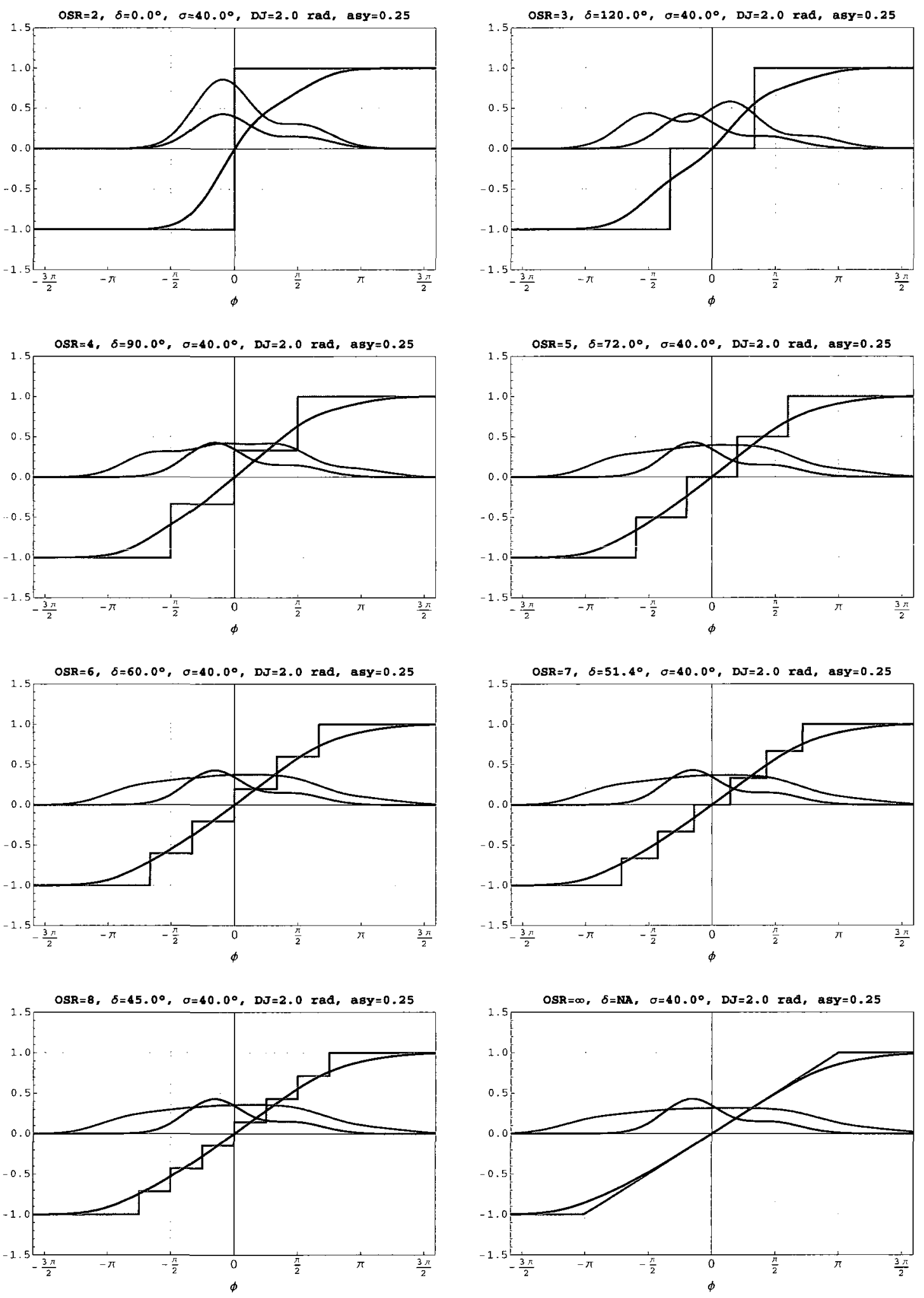

Figure 4.14 Case 6 - Excessive RJ + DJ with asymmetry 

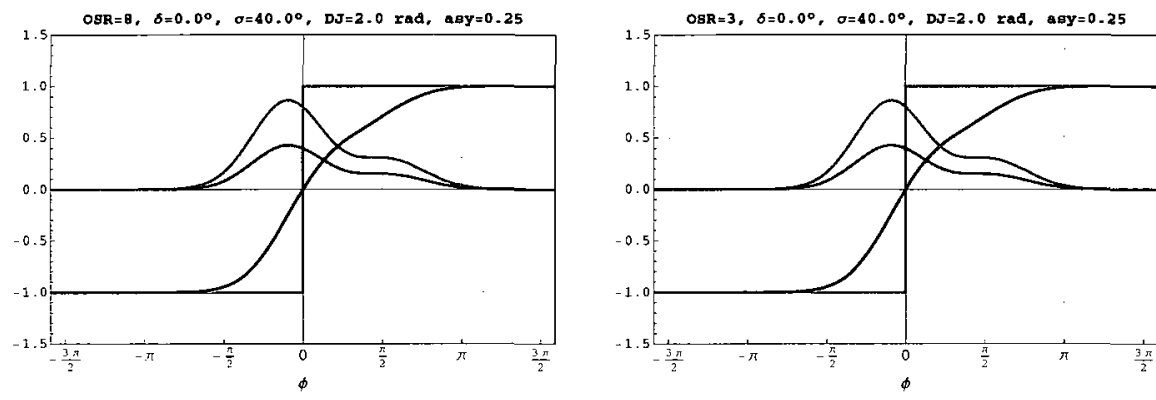

(a) $\delta=0$
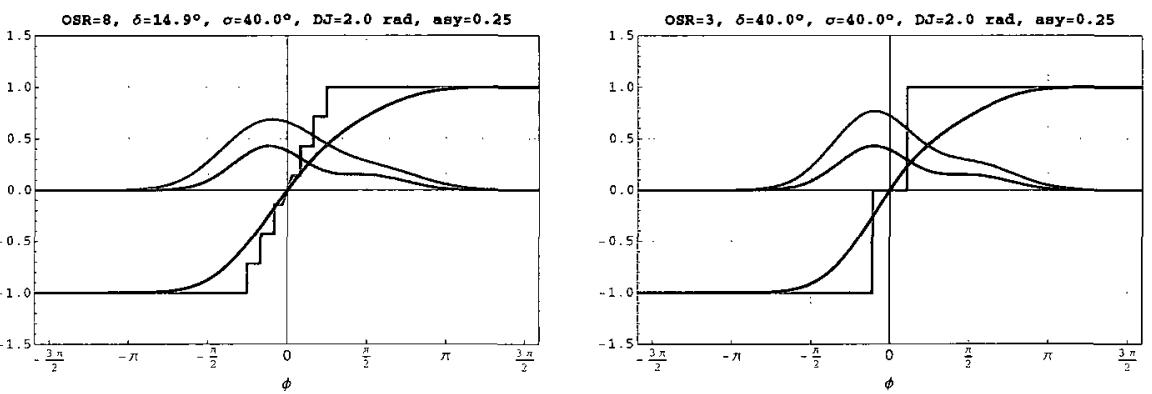

(b) $\delta=1 / 3$ of $2 \pi / O S R$
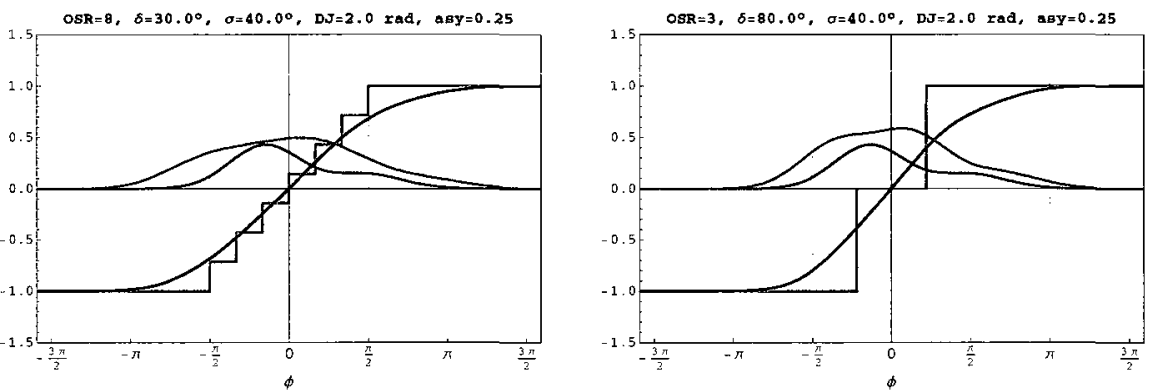

(c) $\delta=2 / 3$ of $2 \pi / O S R$
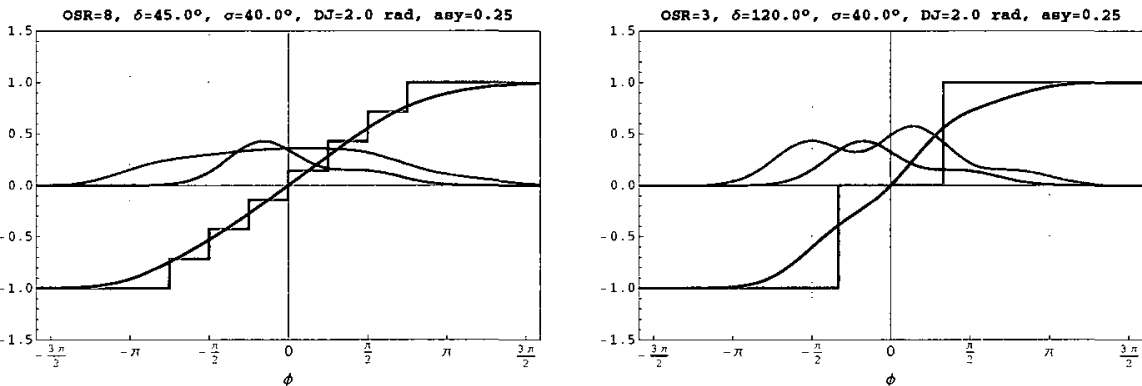

(d) $\delta=2 \pi / O S R$ maximal spacing
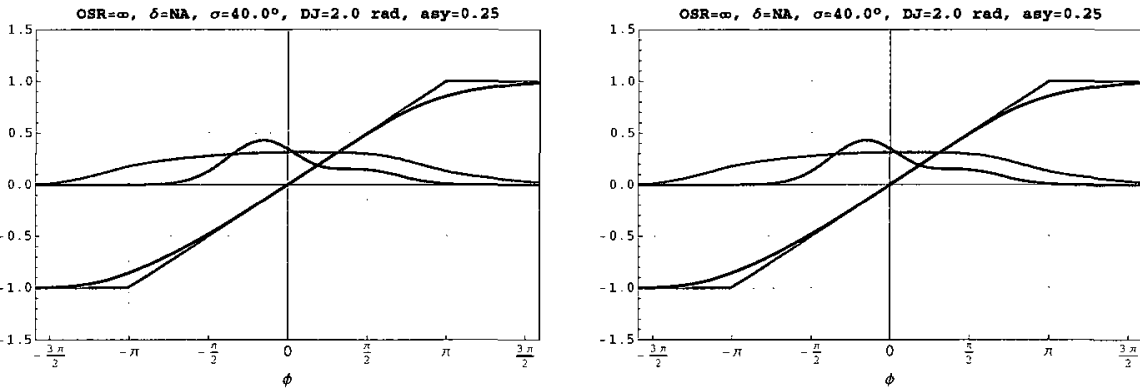

(e) Clip PD for comparison

Figure 4.15 Case 7 and Case 8 - Change of inter-phase spacing 


\subsection{The use of random jitter as a linearizing aid}

In this section, the results are to be read from top to bottom in each column. The plot scale for this section has been modified to show more RJ information. First consider the $O S R=5$ in Figure 4.16(a). The RJ deviation is set to a few degrees case as $\delta$ is swept. A perfect impulse would have simply replicated the PD characteristics. In practice, only clock signals have low jitter-CDR systems seldom have such clean input data [46].
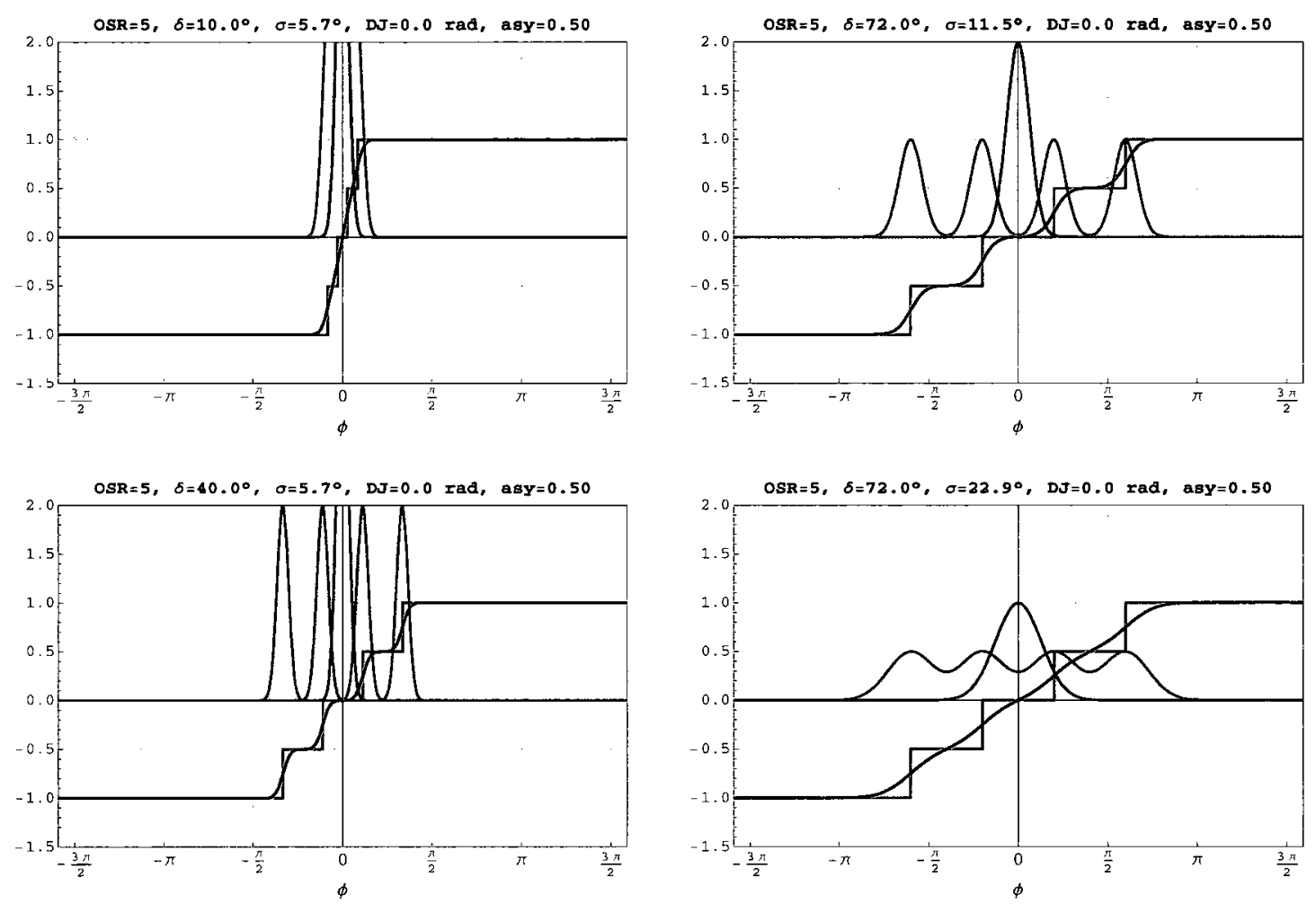

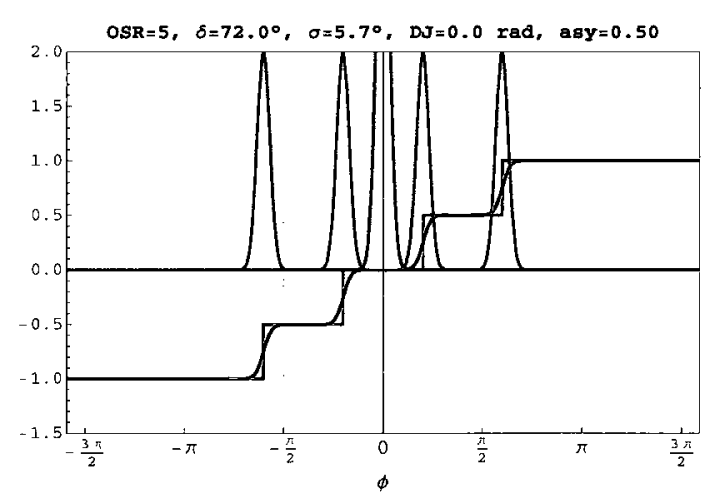

(a)

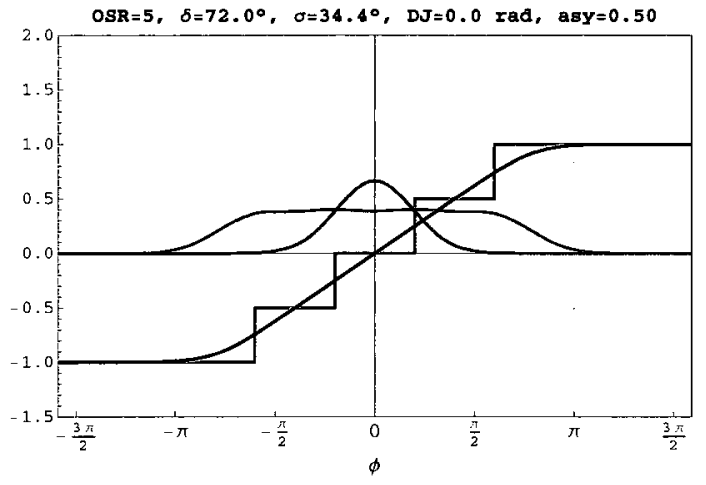

(b)

Figure 4.16 Using RJ to linearize the PD characteristics 
This is not a practical case for CDR design. An aligner can recover the data with sufficient accuracy in this theoretical case. In Figure 4.16(b) more RJ is added (top to bottom), while keeping phase spacing at maximal value. The resulting PD linearization is non-intuitive but is known in conjunction with PLL systems [36]. Figure 4.17(a) and Figure 4.17(b) show the effect on PD linearization (from top to bottom) upon adding modulation style DJ and the more practical case of long-term DJ, respectively.
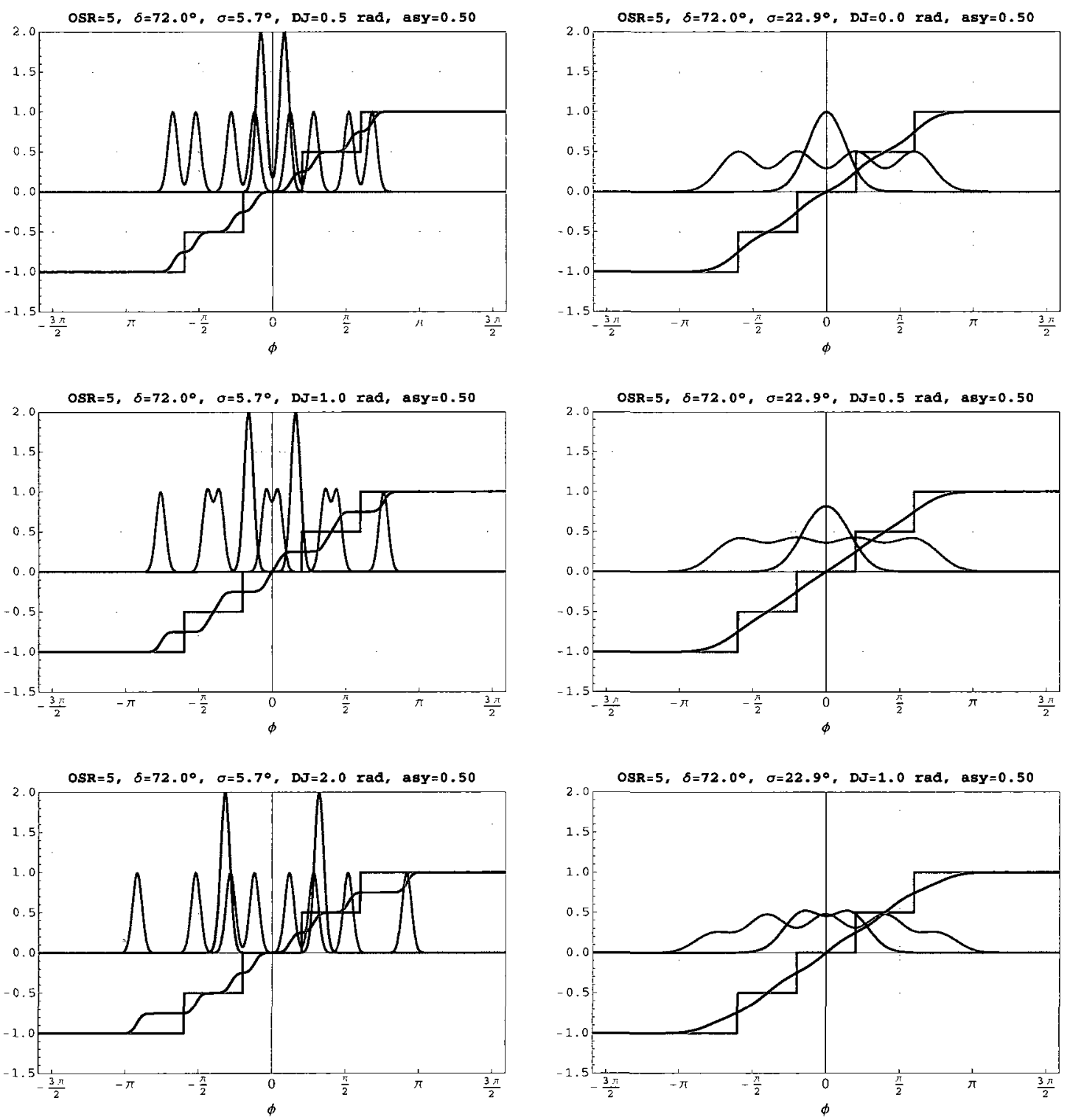

(a) Modulation style DJ

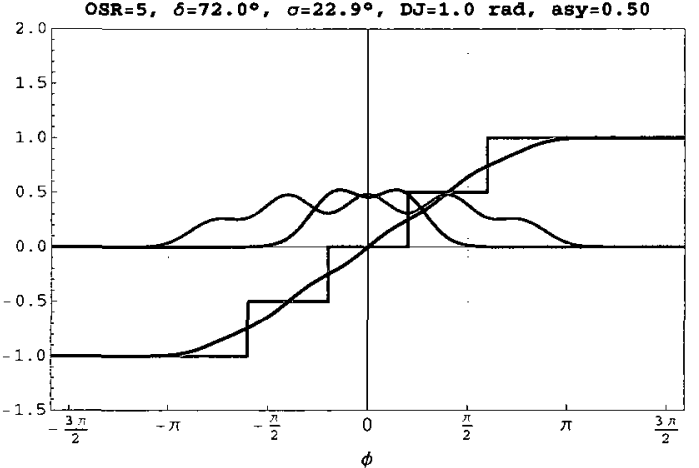

(b) Pattern-related DJ

Figure 4.17 Linearization in presence of DJ 
The addition of RJ and its implications need to be understood since it is precisely what the CDR is trying to reject in order to satisfy a minimum BER specification. This technique cannot be democratized without caution. The addition of $\mathrm{RJ}$ reduces the timing margin and the bandwidth of the CDR but it makes it more robust to variations in the input jitter, especially the asymmetry.

A similar strategy is used to reduce the 'chatter' due to high gain in relay-based control systems. RJ is added to reduce the gain in the vicinity of decision threshold. Relaybased control systems are nothing but bang-bang style systems [14] and the nomenclature is different for electronics and control industries (Ch. 3, [16]). In the chemical industry, such systems have long time constants and oversampling relay systems appear infrequently. For serial links, the time constants are of the order of tens of picoseconds and oversampling is often employed to improve system performance. Using this linearization technique, creative trade-offs can be implemented. For instance, the power of the oscillator can be reduced to allow additional phase noise and in turn extra RJ on the sampling clock. Assuming that CDR architecture uses a local oscillator and the RJ on the sampling clock is not correlated with the incoming data, the RJ variances on clock and data would add together enabling the requisite linearization of the PD characteristics. Such a trade-off requires long counters to identify the input jitter distribution. Since the PD characteristics can be computed analytically, this opens the door to various types of adaptive architectures not possible before.

\subsection{Effect of unequal phase spacing}

Thus far, the phase spacing parameter $(\delta)$ has been treated as a constant. This changes in Figure 4.18 where one of the five phases has a systematic offset. The results in Figure 4.18(a) and Figure 4.18(b) are to be read from top to bottom and depict the cases 
for maximal and non-maximal inter-phase spacing, respectively, for the case of $O S R=5$ in the presence of a static offset on the phase closest to $\phi=0$.
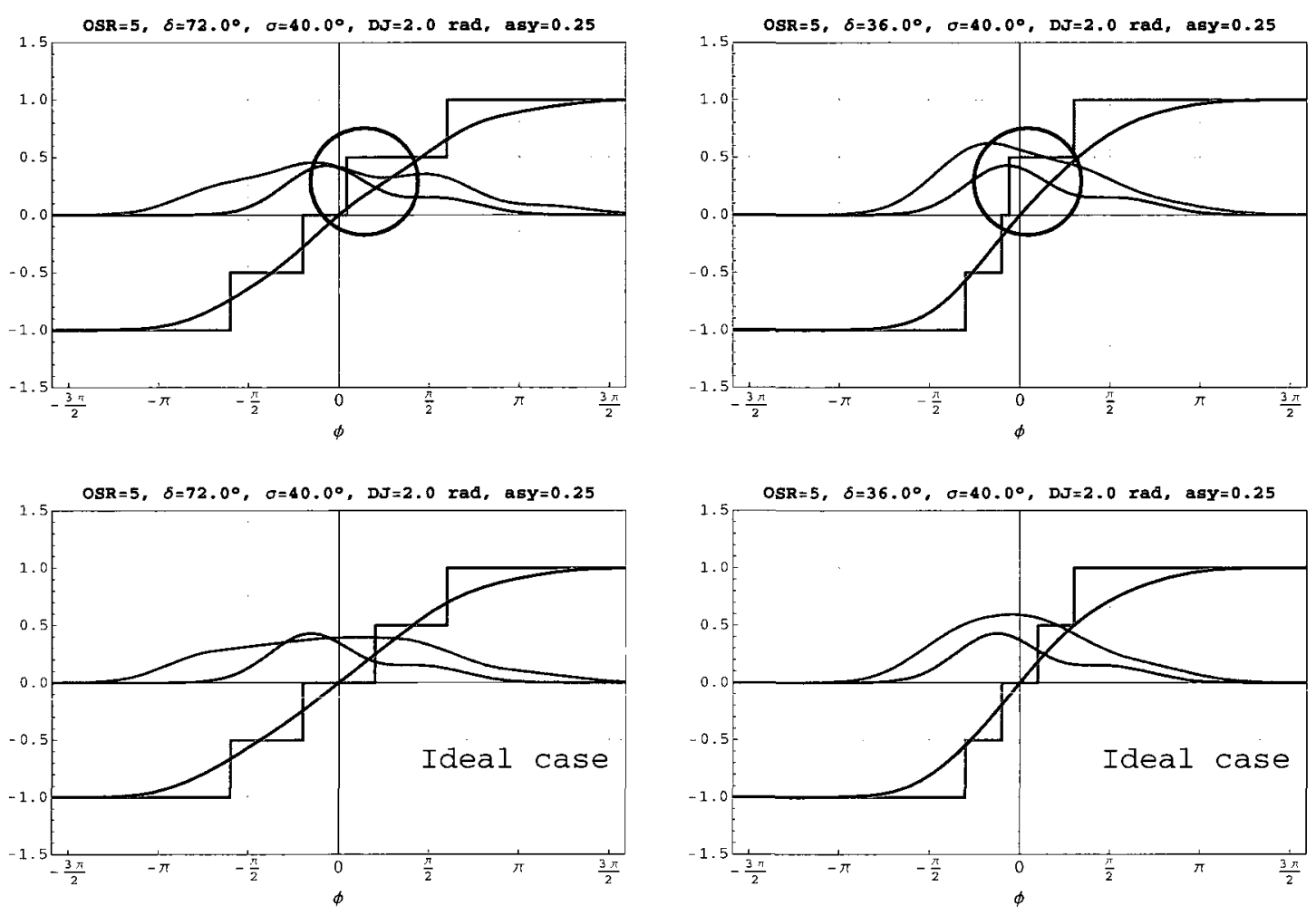

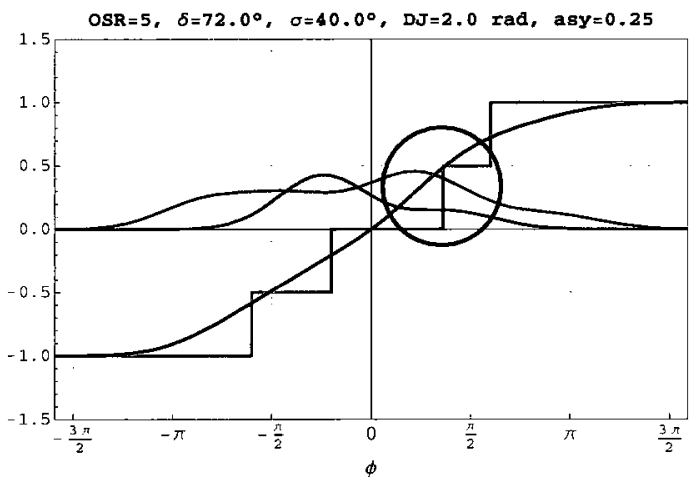

(a)

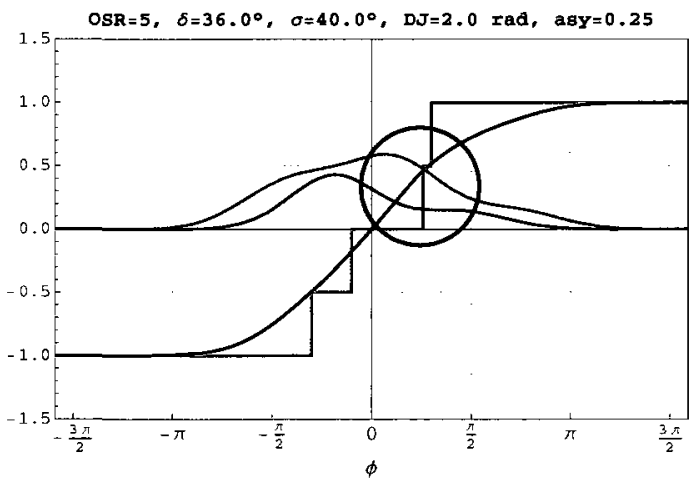

(b)

Figure 4.18 Effect of unequal phase spacing

For an NXO PD, this is a two dimensional problem in addition to the possibilities discussed so far; i.e., which phases have an offset and how much offset is present. Since the averaged NXO PD characteristics are being considered, the effect of RJ on the multiple phases is averaged out. What remains is the static offset caused by improper circuit 
design and incorrect biasing in the oscillator. In order to demonstrate the possibilities, Case 6 is chosen as the base case for Figure 4.18 for a fixed $O S R=5$. The offsets are introduced as the inter-phase spacing $(\delta)$ is set to a maximal value of $72^{\circ}$ (and at $36^{\circ}$ for the second set). For the case of a phase rotating CDR circuit, the static phase offset will be dithered due to an appropriate clock phase getting selected every update cycle. For a nonrotating CDR circuit, more damage occurs to JTOL if the phase with the static offset is closer to $\phi=0$. If the aberrant phase is at the periphery near $\phi= \pm \pi$, then the situation is more manageable. For multiple phases derived from a VCDL inside a DLL, the static offset problem near $\phi=0$ is more relevant. Also using maximal phase spacing provides more cushion as the per-unit phase deviations are smaller. The observations regarding the static phase offsets in PD characteristics are as follows:

- The expected PD characteristics for a lower $O S R$ are affected more than those for a higher $O S R$. Theoretically, for a very high $O S R$, it would be immaterial if one or several phases have a static offset, since each would incur its own non-substantial penalty to the final expected NXO PD characteristics.

- Per unit deviations $(\Delta \delta / \delta)$ need to be minimized for better performance.

- A belated phase step gives rise to a higher PD gain in the vicinity of the anomaly.

- An early phase step creates a lower gain in the vicinity of the anomaly.

- Detailed simulations need to be run for characterizing JTOL and JTRAN in the presence of static phase offsets and those are possible because of the availability of precise equations for the expected NXO PD characteristics.

\subsection{Simulation Flow}

The aim of the interactive software is to choose the oversampling ratio and $\delta$ such that the PD characteristics become linear in the vicinity of $\phi=0$ and taper off thereafter, for the applied DJ and RJ thus resulting in robust CDR performance. From here, there are two possibilities. 


\subsubsection{Transfer function analysis}

First, for the system-level JTOL and JTRAN simulations, the value of $K_{p d}$ can be extracted by evaluating the slope of the generalized NXO PD characteristics at $\phi=0$ for the chosen parameters. A transfer function analysis with constant $K_{p d}$ can be run to see if the JTOL and JTRAN bandwidth constraints would be met. This is applicable to wellbehaved linearized systems. If the slope changes near the origin or diminishes rapidly thereafter, then a circuit style behavioral simulation is the preferred way to characterize the CDR system. Three cases are pictorially depicted in Figure 4.19 that serve as a visual aid only and no particular technical information is emphasized here other than the shape of the expected PD gains and their utility for the transfer function style analysis.

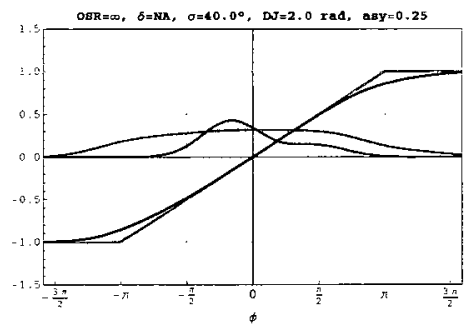

(a) Applies to linear PDs

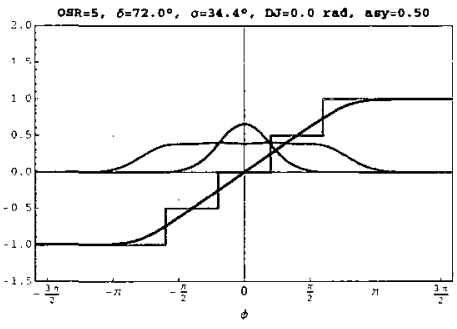

(b) Good approximation

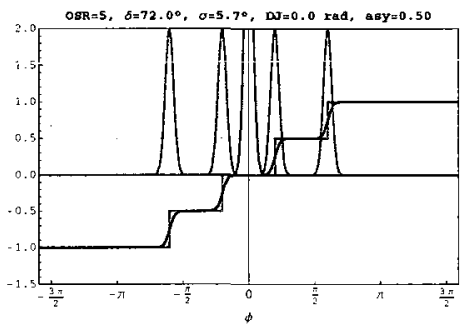

(c) Inaccurate

Figure 4.19 Validity of transfer function analysis

\subsubsection{Behavioral Modeling}

The parametric equations provided by the software for the expected NXO PD characteristics are programmed into the Verilog-AMS simulator by applying the substitutions shown in Equation (4.6) and Equation (4.7) such that $\operatorname{erfc}(x) \Rightarrow \operatorname{erf}(x)$ and $\operatorname{erf}(x) \Rightarrow \tanh (x)$. The aim of this effort is to generate a visual catalog of various PD characteristics vs. the JTOL and JTRAN curves-such that an understanding of the effects of PD characteristics upon the JTOL and JTRAN performance is facilitated. The results have been plotted using Mathematica ${ }^{\circledR}$. The Verilog-AMS simulator lacks the solve(.) functionality—so calculations for $\phi_{c}$ are done in Mathematica prior to equation export. 


\subsection{Effect of PDs on CDR performance}

Several test cases will be studied in this section to demonstrate the possibilities and to develop an understanding of how the expected NXO PD characteristics affect JTRAN and JTOL performance of the CDR system with all other components remaining invariant. If the performance of other components in the loop is dependent on $\mathrm{DJ}$ and/or $\mathrm{RJ}$, then more modeling effort is required in conjunction with those components and the loop itself. The expected NXO PD equations can only provide calculated benefits when used in conjunction with a proper behavioral model. The import of the interactive, symbolic software is the availability of the precise NXO PD equations for various jitter conditions.

\subsubsection{Base Results}

The phase domain simulation methodology follows the Kundert's phase domain CDR model and the associated JTOL testbench with some adjustments to the model [35]. Also a JTRAN testbench has been set up independently. The industry-standard VerilogAMS simulator is used. The base results for JTOL and JTRAN are shown in Figure 4.20 for two values of the VCO gain to establish a reference point. No effort is made to optimize the base CDR for any particular specification. Higher loop gain improves the CDR bandwidth and JTOL but deteriorates the JTRAN characteristics, so a trade-off is sought.

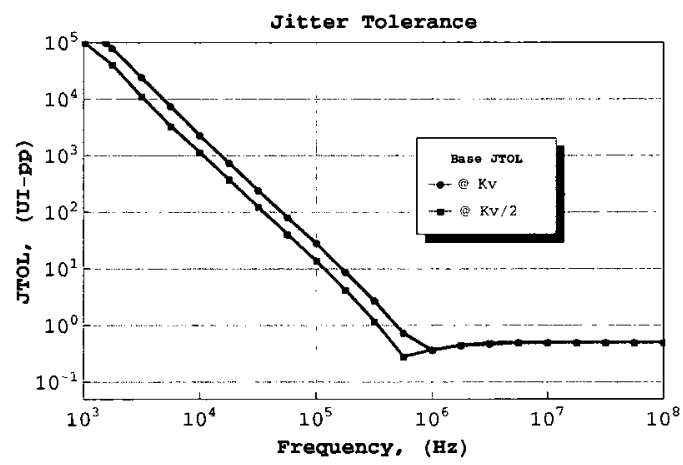

(a) JTOL

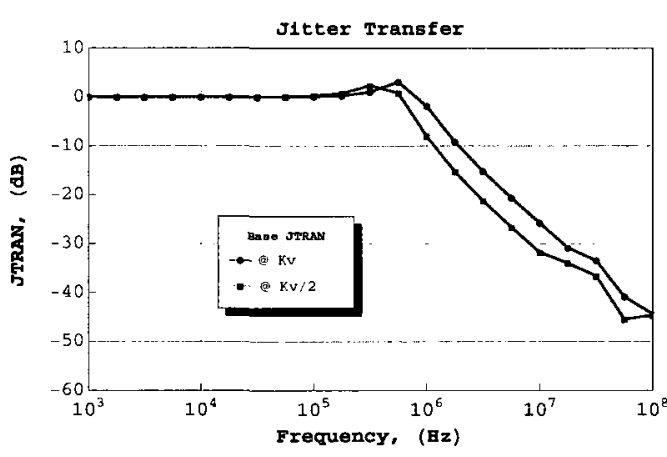

(b) JTRAN

Figure 4.20 Base results for JTOL and JTRAN 


\subsubsection{Effect of changing $R J$ and DJ separately}

Figure 4.21(a) and Figure 4.21(b) show the effect of changing RJ and DJ respectively upon the JTOL and JTRAN of the reference BBPD CDR. The results are to be read from top to bottom in the same column.
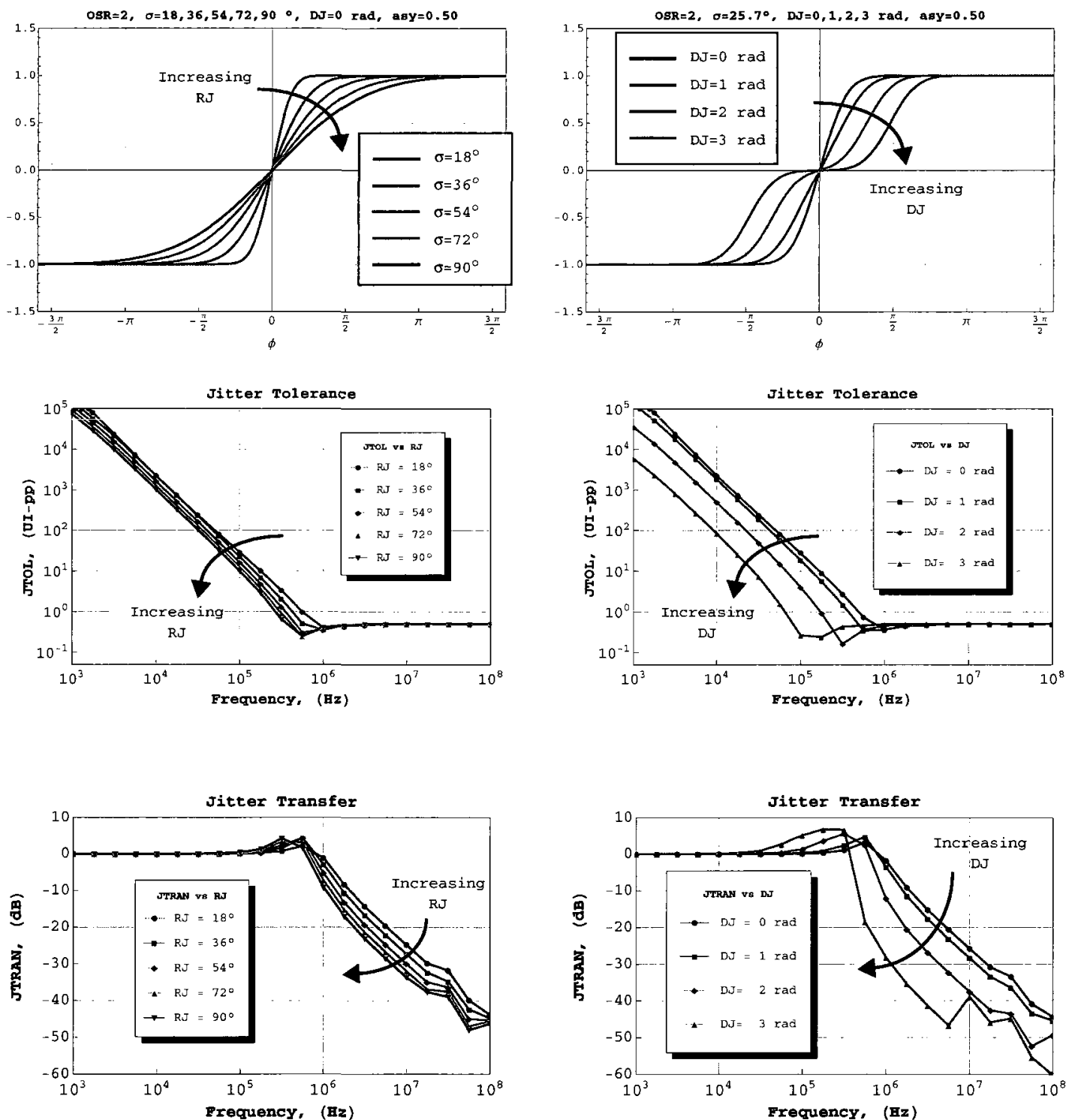

(a) Changing RJ only

(b) Changing DJ only

Figure 4.21 Effect of jitter distribution on JTOL 
It is apparent that the bandwidth of the BBPD CDR is dependent on the RJ and DJ present in the system and can fluctuate heavily. As RJ is increased (Figure 4.21(a)), the bandwidth of the BBPD CDR is reduced correspondingly. For the JTRAN case, a higher bandwidth results in less peaking and a lower bandwidth accentuates the jitter peaking. A typical CDR needs to pass the specifications for bandwidth, locking range and high frequency JTOL. The JTRAN specifications are important for repeater applications.

Figure 4.21(b) shows the case where the DJ is increased while RJ is held constant. A deadzone results in the BBPD characteristics. This signifies the lack of edges in a statistical sense near $\phi=0$ since the modulation moves from side to side with a deterministic resolve. The bandwidth is deteriorated as well as the JTRAN peaking performance. Hence for the case of DJ, a frequency detection mechanism inside an integral loop would be required to counter the peaking and track the data. The BBPD case has been chosen to highlight the sensitive nature of the CDR to routinely present anomalies. One might be tempted to generalize this behavior to oversampling or digital CDR circuits, but oversampling can alleviate this design challenge. 


\subsubsection{Robust operation with a higher OSR}

Figure 4.22 shows the case where a ripple in PD characteristics can be mitigated by the use of RJ as a linearizing aid as explained in Section 4.13. This is being used to study effects that are hard to understand and characterize. A brief explanation follows.
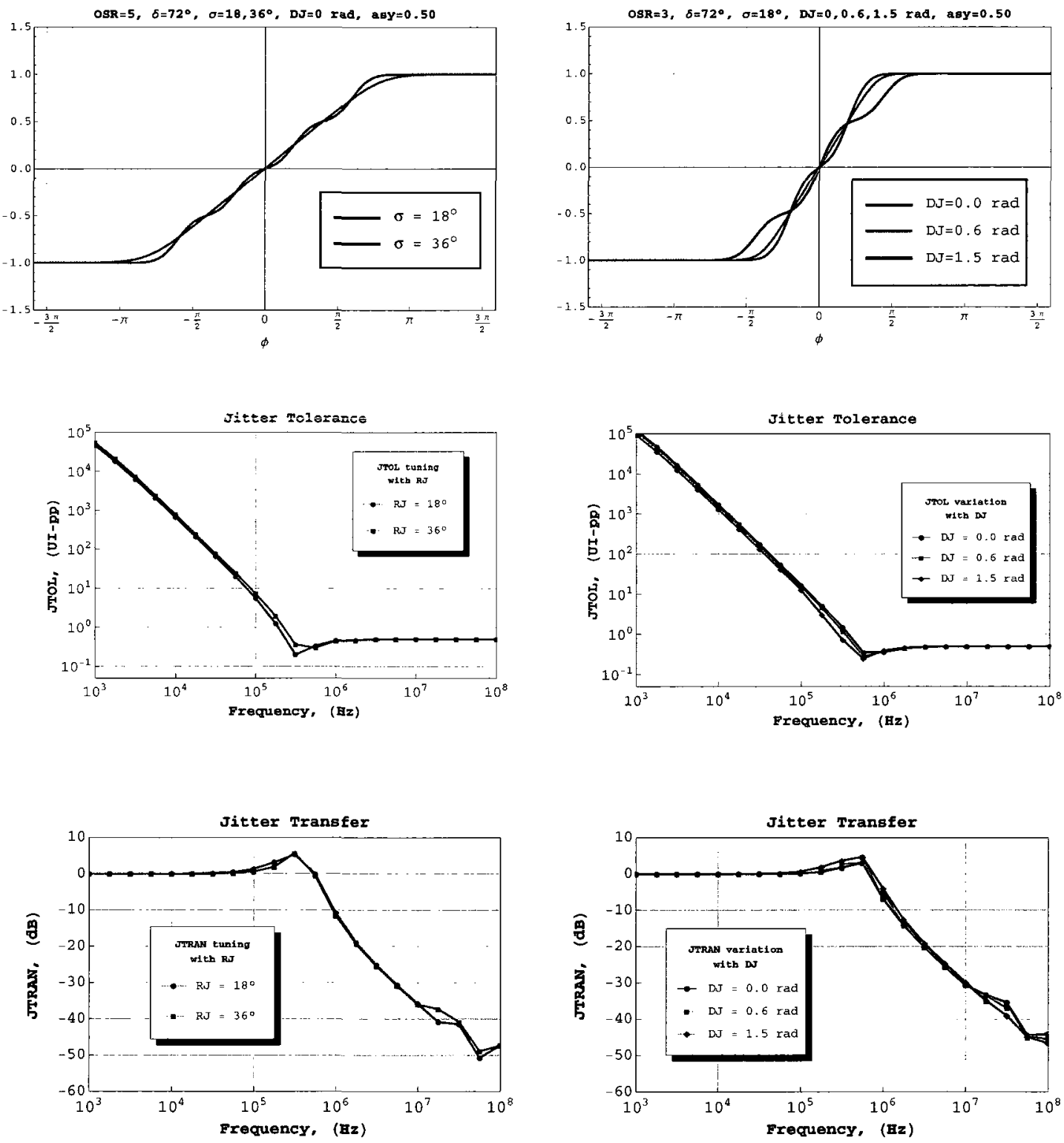

(a) Maximal phase spacing

(b) Non-maximal phase spacing

Figure 4.22 Robustness due to a higher oversampling ratio 
This particular comparison has been included here as a gentle reminder that intuition might not lead very far when hard non-linearities are at play during system-level CDR design. For certain combinations of RJ and asymmetric DJ, the PD gain at $\phi=0$ may be higher or lower than the desired value while maintaining the same average slope. This results in subtle changes in the bandwidth and behavior of the CDR circuit. Figure 4.22(a) and Figure 4.22(b) show two cases for maximal and non-maximal phase spacing. The JTOL bandwidth is necessarily reduced if the slope at $\phi=0$ is reduced; but the tracking performance might not be too far off since the average PD gain remains the same. Without the availability of exact NXO PD equations, it would be impossible to study this effect. Further, a higher $O S R$ stabilizes a CDR bandwidth in the presence of RJ and DJ as can be seen from Figure 4.22.

The modulation of PD gain can potentially show up during testing as the test equipment lets in unclassified RJ and DJ into the device under test. During prototype testing, this can result in a different performance from hour to hour-something that can be quite annoying and costly.

In summary:

Oversampling helps with the linearization of PD gain, stabilization of CDR bandwidth and provides robust operation under asymmetric jitter conditions.

\subsection{Miscellaneous topics}

This section discusses miscellaneous topics related to the discussion so far.

\subsubsection{Using JTOL curve to debug CDR or PD anomalies}

Continuing with the same theme, the reverse question may also be asked. If a deadzone in PD characteristics leaves a certain stamp on the JTOL curve, is it possible to find the PD deadzone from an available JTOL curve? 
As a start, this requires a reference JTOL curve for the CDR. So for a first time design, it is not a possibility. Second, if the exact conditions for RJ, DJ and asymmetry can be replicated, then JTOL characteristics can perhaps be replicated. Achieving that in real life is difficult due to PVT variations, calibration cycles, test equipment setups etc. Thus, the effectiveness of the question initially posed is limited to simulation only.

It has also been assumed in the preceding discussion that every other component in the CDR remains invariant. An effect similar to the one shown in Figure 4.22 can be observed if the loop filter characteristics develop a 'wavy' look for any reason. While hard to theorize on what such a filter looks like in practice, it is easy to see that the reverse engineering using the final JTOL curve is not straight-forward. This topic can benefit from further research into specific cases and might have some utility in specific cases.

\subsubsection{The case of colored noise in control systems}

In control systems literature, the effect of colored noise on detection systems is frequently studied and this terminology is not used in the SerDes area. Some explanatory comments are in order.

There are two distinct problems in the SerDes design-Equalization and recovery of clock and data. If the colored noise affects the amplitude of the signal, then the DFE has to manage it. This is outside the scope of this dissertation.

After equalization, if the colored noise affects the phase of the incoming data then it has to be dealt with in terms of the jitter-the metrics for which are well developed in the SerDes literature. This jitter is measured using various instruments as briefiy discussed in Section 3.14.4. There is no joint study available, to the best of author's knowledge, that can be used to map the nomenclature across the control systems and SerDes disciplines. The main reason is that the SerDes area is relatively recent and has its own terminology evolving continuously. In short, colored noise may be represented by (asymmetric) DJ that has a limited spectrum. 
The interactive software can handle Gaussian RJ and DJ. A clarification is in order regarding the quantity of the DJ that the user must take care not to exceed. As flatness occurs on the jitter histogram, it represents the pattern-related jitter that is deterministic; since many patterns would cause the same jitter for a long PRBS pattern. This happens in the long-term-a duration that is a sub-multiple of the PRBS run-length. This means that many such patterns happen during a run-length but there is no shortage of edges in the vicinity of the $\phi=0$ point. This is known as long-term, pattern-related DJ.

Short-term periodic jitter such as sinusoidal jitter, however, causes a deadzone near $\phi=0$ and represents a case where there is a dearth of edges near the locking point as the jitter moves the edges in a specific direction over a short period of time. This type of jitter is added using a JTOL simulation - and no double-counting should be done. It is a bit more clear now why colored noise is hard to map to various types of jitter and this topic can benefit from cross-disciplinary research.

\subsubsection{Metastability in PD flipflops}

Metastability has been studied in the literature [115][116]. Its effect has been mentioned in conjunction with the current topic [33][35]. It is a research problem. The first part is the accurate characterization of metastability for various flipflop architectures as used in a CDR configuration. This research on metastability is outside the scope of this dissertation. The second part is including it within the NXO PD methodology. If the effects of metastability can be parametrized in terms of the DJ, RJ and asymmetry, then the mechanism to include such effects has been made available through this work. If metastability is independent of the jitter distribution (which is highly unlikely), then a direct addition of variances can be employed else the covariance of metastability and the incoming jitter will also have to be incorporated. This topic needs further research since metastability is not going anywhere and might as well be understood and exploited to improve CDR design. 


\subsection{Summary}

This chapter has presented a unified theme and mathematical analysis of the PD mechanisms for CDR circuits. The analysis for any PD mechanism-from a BBPD to a general NXO PD and on to a linear PD — is given by the same mathematical procedure. As more sampling phases are added, the performance of an NXO PD approaches that of a linear PD in the limit. It makes intuitive sense-since a linear PD detects the phase difference with infinite resolution and provides proportional correction. If the $O S R$ could be set to infinity, then the NXO PD performance would also gain independence from the shape and magnitude of the input jitter. Linear PD design is not feasible for Gbps data rates. The optimal oversampling ratio and inter-phase spacing for a PD can be found analytically in the presence of jitter - such that the CDR performance becomes robust using those parameter choices. This is accomplished using the interactive features of a program written using a symbolic and an interactive mathematics software known as Mathematica ${ }^{\mathbb{B}}$. Using this interactive program, the variables $O S R$ and $\delta$ can be adjusted for optimum PD characteristics in the presence of jitter. A behavioral simulation flow is proposed to evaluate the effects of PD characteristics on JTOL and JTRAN performance. This facilitates the design of oversampling CDR systems and is a major contribution of this dissertation. 
"We dance around in a ring and suppose, But the Secret sits in the middle and knows."

Robert Frost, "The Secret Sits"

\subsection{Chapter overview}

This chapter presents a novel Multiple-Rotating-Clock-Phase (MRCP) data recovery (DR) architecture after reviewing, in further detail, the relevant digital CDR literature. Simulation and measurement results for the proposed novel DR architecture are presented. An FPGA implementation is used as a proof-of-concept vehicle to verify MRCP-DR functionality using Verilog HDL.

\subsection{Digital CDR design-most important point}

The central theme of the ongoing discussion about digital CDR systems is that the digital techniques facilitate abstractions that streamline product development. Digital circuits can be synthesized and laid out automatically, tested using a BIST, re-used and configured on the fly to deliver optimum performance. These are clear advantages over the conventional analog CDR systems.

Having established the digital CDR architecture as a bona fide contender in the CDR design space and having analyzed the effect of $O S R$ on PD performance analytically, the stage is now set to implement an all-digital CDR circuit that can utilize today's FPGA technology. A DCO is not available on FPGAs as a synthesizable block as of 2010 so a proof-of concept strategy is adopted with a simpler architecture for multi-phase clock 
generation. Implementing a DCO or a DDFS on the FPGA does not form part of the experimental work.

From the generalized NXO PD analysis, it is clear that the advantages that accrue with oversampling disappear if the phase spacing parameter $\delta$ is set to zero reducing the NXO CDR circuit to a BBPD CDR circuit, both mathematically and physically. If $\delta$ is set to a maximal value, lowest PD gain results for the chosen $O S R$ but with the most extended linear range possible around the sampling point for the given input jitter. This results in a robust JTOL and JTRAN performance.

\subsubsection{Jitter at PD input}

To recap, adding $\mathrm{RJ}$ reduces $\mathrm{PD}$ gain but increases the linear range for the BBPD gain as seen in Figure 4.21(a). The addition of DJ at the BBPD input changes the situation a bit as seen in Figure 4.21(b). In this context, there are two scenarios. If it is a repetitive modulation input, let's say $50 \mathrm{kHz}$, it would shift the edges to either side of the sampling point, 50000 times per second. If it is pattern related, it could be happening tens or hundreds of times during the run length of the PRBS. Hence the time constants are order of magnitudes different for short-term DJ and long-term DJ. The former scenario results in a concave DJ characteristics (Figure 4.12) whereas a minor flattening occurs on the jitter histogram for the latter case (Figure 4.11).

\subsubsection{Phase tracking vs. frequency acquisition}

Comparing the clip PD characteristics with the BBPD characteristics, it can be seen that the hand-off to the frequency mechanism occurs at the edge of the UI for a linear PD. For the BBPD or a $2 \mathrm{XO} \mathrm{PD}$, this range shrinks to zero. This means that the BBPD treats every input data edge as an FD problem and undertakes corrective action. Indeed, a first-order BBPD CDR corrects the frequency of the oscillator every cycle to remain in sync with the incoming data resulting in small voltage jumps at the $V_{\text {cont }}$ line [32] even 
under lock. A second-order BBPD adds an integral path to acquire lock and to track frequency drifts. The first-order BBPD is an inadequate choice for a CDR system and a second-order circuit is a more practical implementation. The integral path provides a longterm outlook that takes care of the DJ. The point of this discussion is that the PD is not an FD device and should not be expected to do any frequency acquisition work; its job extends as far as tracking data is concerned. The transient acquisition performance of BBPD-based PLL circuits has been discussed in the literature [41].

By adding a ternary output for the case of no transition, the oscillator frequency is kept constant in an Alexander PD system resulting in less disturbance on the $V_{\text {cont }}$ line. This is a step in the right direction and two relevant scenarios occur. For analog CDR systems using a ternary PD, the V-I converter is not disturbed in the event of no transitions and the LF droop happens due to CP leakage only. For digital systems incorporating a digital filter and a DCO, the varactors are randomized as usual but the value of capacitance remains unchanged during a CID run. The modulation style DJ needs to be detected and corrected otherwise deterministic errors accumulate fast and the circuit is rendered nonfunctional. Oversampling PDs are better than a BBPD when it comes to the visibility of the edges during the UI. The wander tracking and HF JTOL performance can be separately optimized and studied [48].

\subsection{Oversampling architectures and complexity}

A mechanism to linearize the BBPD characteristics is to be sought such that the hand-off to the FD mechanism occurs closer to the edge of the UI. This is made possible by oversampling as realized in conjunction with the analysis of NXO PDs earlier. There is no way to use ' $n$ ' out of ' $m$ ' phases until ' $m$ ' phases have been generated and a CPP, an AMS mechanism or a register bank has enabled such a selection [45]. If the phase spacing is such that the entire UI is not covered, then there must be a way of tracking the farthest edges of jitter within the UI and positioning the pointers accordingly [49][50], which 
increases the AMS design complexity. If the entire UI is covered by the PD then the phases at the edges, it can be argued, provide little additional value since most of the edges are concentrated around the sampling point. It makes sense to concentrate fewer phases at the center and adjust $\delta$ to achieve the design objectives. Having a smaller number of phases spanning the centre of the bit degrades performance, and phase spacing for the two outer edges can only be adjusted if there is an AMS mechanism [50] or by selecting ' $m$ ' of ' $n$ ' phases with digital circuitry using a clock phase pointer (CPP) [48]. A larger OSR enables smaller phase jumps at the output during updates. In short, more phases are desired as long as someone else pays for them-a typical engineering trade-off situation. Continuous steering of fewer phases and having a multitude of phases to select from have resulted in the VI-3XO [50] and ET-DR architectures [48] respectively. These architectures optimize BER for the case of asymmetric jitter. The underlying concepts behind the VI-3XO CDR and the ET-DR architectures are shown in Figure 5.1.
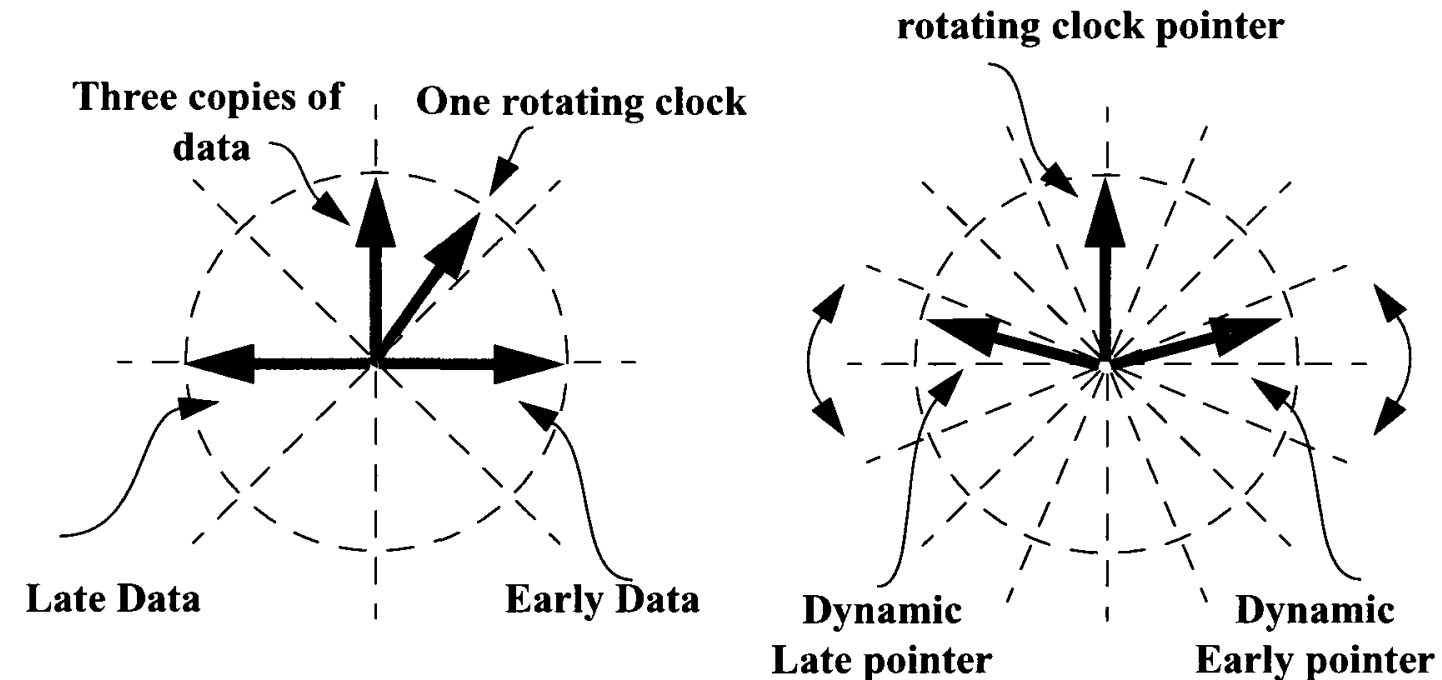

(a) Eye-tracking concept Fixed decision threshold

(b) Variable-interval oversampling concept

Figure 5.1 Eye-tracking and and variable-interval oversampling CDR concepts 
Within the oversampling CDR architectures, the BOS CDR circuit relies on nominally equidistant phases. The BOS CDR circuit is inefficient in terms of power and area as discussed earlier.

Like most engineering products destined for mass consumption, the oversampling CDR systems gravitate toward cost reduction by using the migratory theme - that of ana$\log$ to digital thinking. Oversampling is difficult at higher frequencies so it is not a fix-all solution. However, if a capable multi-phase oscillator is available in a library or on an FPGA, then it can be used to implement effective CDR systems with repeatability. This allows the large system integrators and IP designers to focus on the CDR performance, rather than the mechanics of the oscillator design.

\subsection{Eye-tracking DR architecture}

As a first step, the design space for the reference ET-DR architecture was explored using MATLAB [17] and Verilog-AMS models [18] prior to the introduction of the novel MRCP-DR design. Three parameters were selected and swept for this purpose; the analog delay $\left(T_{\text {del }}\right)$ between copies of data, the number of available clock phases $(N)$ and the update period in bits $(M)$. The terminology and the plots in this section have been directly adopted from a prior publication [17].

The effect of $T_{d e l}$ shown in Figure 5.2(a) and Figure 5.2(b) requires explanation and agrees with NXO PD theory presented earlier. Smaller interphase spacing $(\delta$, called $T_{d e l}$ here) simply diminishes the ability to see the edges since the entire UI is not covered by a limited number of edges and thus reduces the tracking ability and CDR bandwidth. The high-frequency (HF) JTOL is improved for the same RJ since less corrections are attempted. The inability to detect edges, however, is not a desired outcome. The reverse happens for larger values of $\delta$. An optimum value can be chosen, but it needs to remai 
PVT invariant or the JTOL would vary as shown in the analysis on NXO PDs in Figure 4.18.

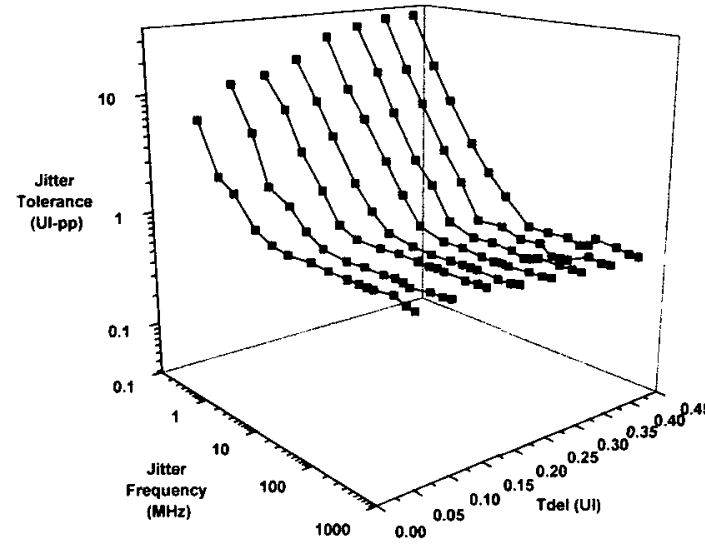

(a) JTOL vs. $T_{d e l}$

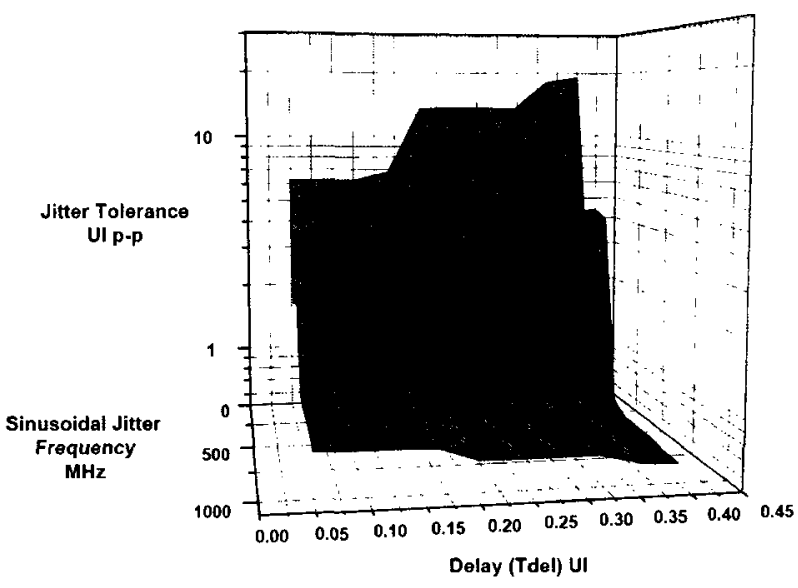

(b) Smoothed trend

Figure 5.2 Effect of $T_{d e l}$ on JTOL

The number of available phases $(N)$ reduce the JTOL for the same update period as shown in Figure 5.3(a). The update period $(M)$ reduces the tracking bandwidth in a $\log _{2}$ fashion as shown in Figure 5.3(b). More clock phases require a shorter update period to achieve the same jitter tracking ability.

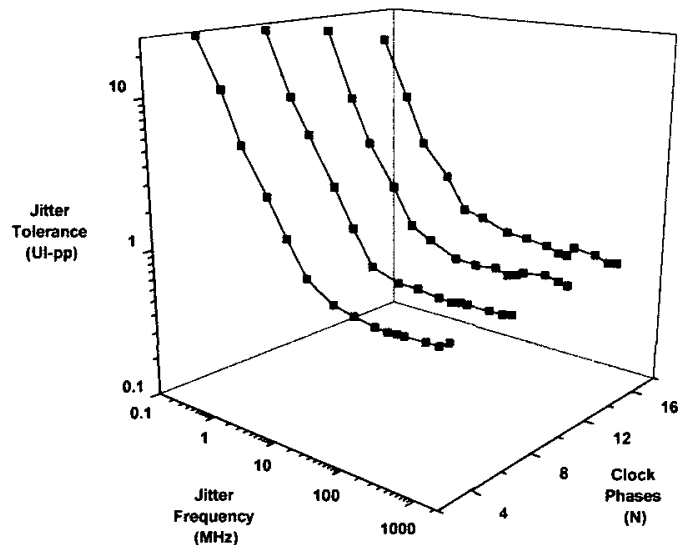

(a) JTOL vs. clock phases $(N)$

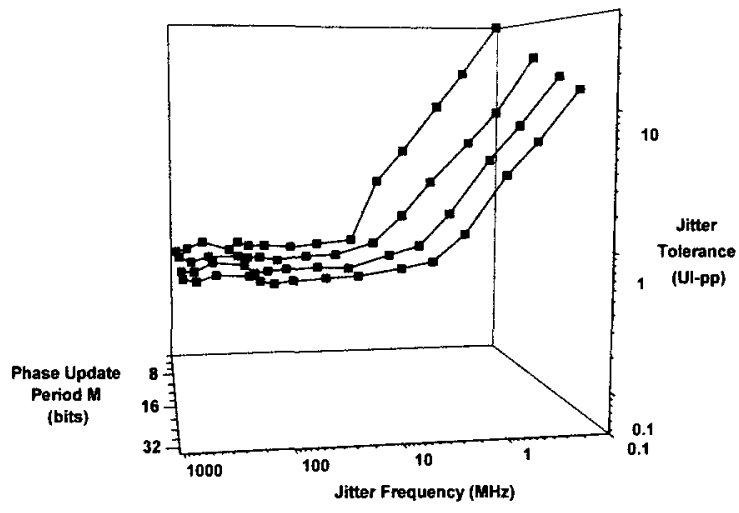

(b) JTOL vs. update period ( $M$ )

Figure 5.3 Effect of $M$ and $N$ on JTOL 
The number of clock phases are fixed by oscillator design. In order to have a continuously variable $\delta$, the VI-3XO CDR architecture is required with all its AMS complexity. A simpler selection of the center phase is more economical. The update period can be manipulated digitally and the CDR circuit does not have to correct the sampling phase every cycle in order to meet BER specifications. The trade-offs that occur due to the choice of inter-phase spacing $(\delta)$ (or $T_{d e l}$ ) are PVT-dependent. The RJ, DJ and the asymmetry parameters make this situation more unpredictable. No two PD designs would give similar NXO PD equations for variations in $\delta$. The solution to this problem is to make $\delta$ largely invariant to the PVT variations. This implies that the phases should be tapped from the clock phases generated inside a feedback style PLL architecture. To accomplish this, the roles of clock and data had to be reversed. A novel DR architecture was thus proposed.

\subsection{Block diagram of the MRCP-DR architecture}

The major block diagram level differences between the ET-DR and MRCP-DR architectures are shown in Figure 5.4 [20]. Instead of rotating one clock phase to track the data, the idea is to rotate three clock phases to create a stable window for data detection. Thus, the JTOL performance becomes robust against variations in $\delta$ over PVT corners.

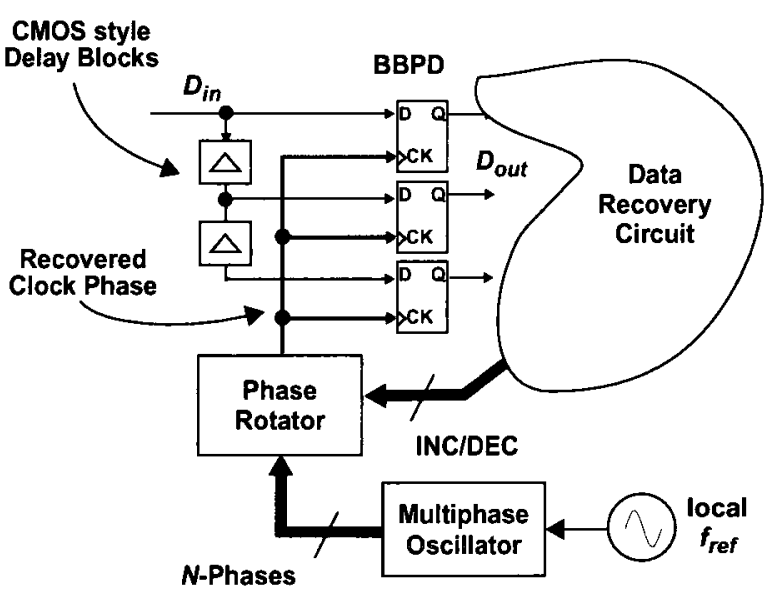

(a) ET-DR [Miki]

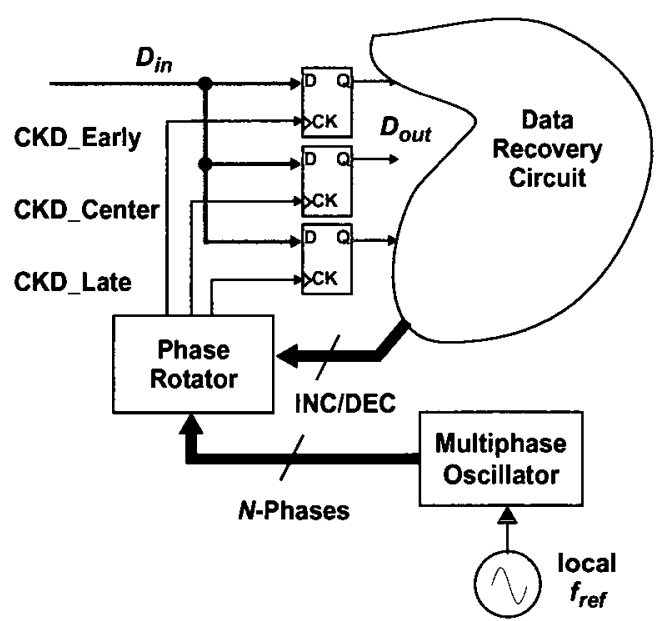

(b) MRCP-DR

Figure 5.4 ET-DR vs. MRCP-DR architecture 
Figure 5.5 shows the principle of operation of the ET-DR and the MRCP-DR architectures. The data detection window is created using three stable clock phases instead of creating three delayed copies of the incoming jittered data.

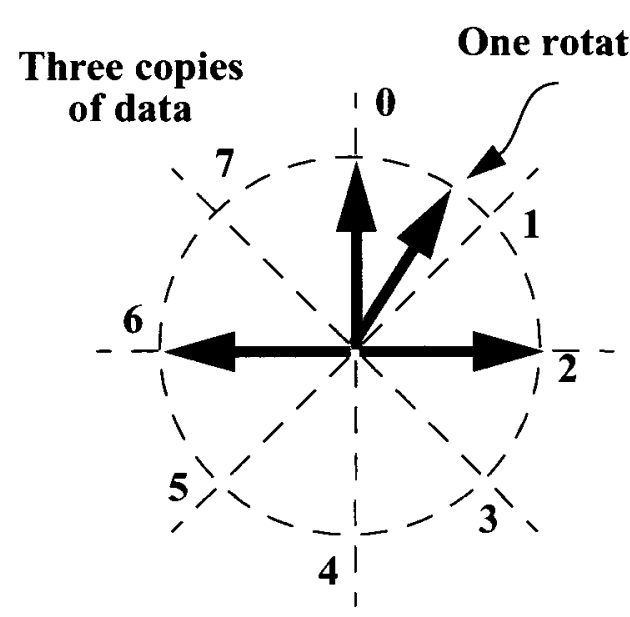

(a) ET-DR

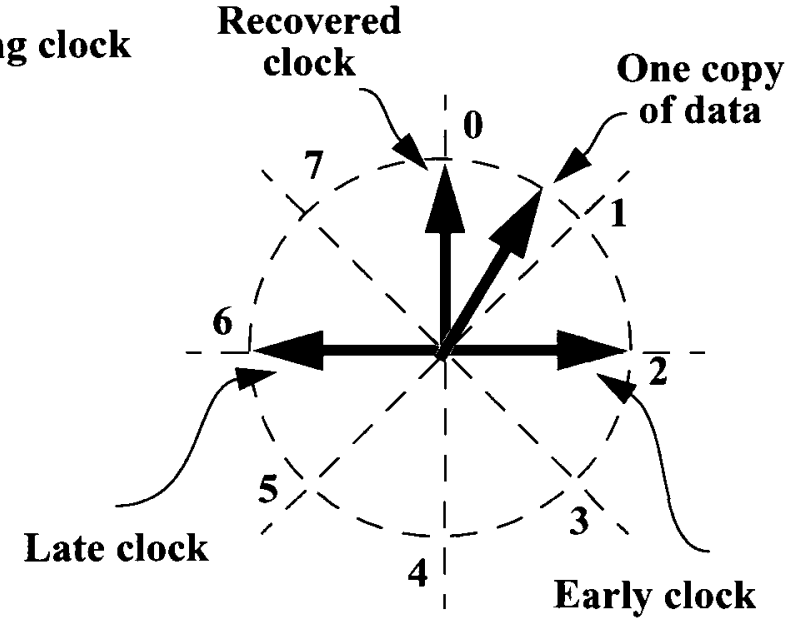

(b) MRCP-DR

Figure 5.5 Three data phases vs. three clock phases

\subsection{Founding thoughts for the MRCP-DR Architecture}

The founding thoughts for the MRCP-DR architecture are listed below:

- Miki's architecture [48] has analog delay elements that are not easily portable. Recent research in this direction has generated high resolution time-to-digital converters [77] but the area required is comparable to that of the digital CDR core (reported in [48]) and the current consumption is quite high.

- A multi-phase oscillator working in conjunction with this architecture already has delays between the clock phases. Hence, if the roles of clock and data can be switched, then oscillator phases can be re-used.

- As an added benefit, the phases derived from a PLL- or DLL-based multi-phase clock generator are PVT invariant compared with the free-running oscillator scenario or due to the use of analog delay elements. 
- Instead of creating three copies of jittered data, three clock phases are tapped to create an edge-detection windows and subsequently rotated to track the data.

- If the data rate changes for a multi-protocol SerDes, then re-tuning the oscillator to the new bit rate using an FD mechanism provides the right delays automatically making the architecture more flexible and useful. In Miki's paper, the analog delay values would have to be tuned to accommodate a change in bit-rate.

- The delay elements are not offered as standard components on FPGAs and their inclusion in libraries is cumbersome. Multi-phase oscillators are not standard fare on FPGAs either, but PLL and DLL offerings are more commonly available.

- Delaying the data two times in order to create three copies creates its own ISI and group delay issues. Since a CDR circuit is positioned after the DFE, this added ISI would add directly to the incoming jitter and the DFE would not be able to help it.

- The CML delay elements need to have no bandwidth limitation at speed. Otherwise, the chain of delays will have progressively worsening jitter for the copies of data. This introduces more uncertainty for the ET-DR circuit.

- If the three CML delay elements suffer a dissimilar common mode voltage change, then unrepeatable JTOL and JTRAN characteristics results. This can be especially problematic during testing, especially when many lanes are involved and several bit-rates must be accomoadated in a multi-protocol SerDes product.

- The design of the CDR circuit should be decoupled from the design of the multiphase oscillator.

- If an FPGA provides a multi-phase oscillator, then the remaining CDR circuit can be automatically synthesized.

- The algorithms and architectures for the loop filter can be changed on the FPGA, whereas it is difficult to do so on a custom ASIC. Hence different filter algorithms can be studied in hardware for different bit rates prior to an ASIC implementation or mass production.

- A switch matrix is required to implement a multiple-lane DR architecture using the MRCP DR technique. 
- The MRCP architecture requires power to rotate the two extra phases. It is, however, smaller than the power required to create analog delay elements or precision time-to-digital converters [77]. It does not need much extra area as well.

- For the MRCP-DR architecture, power is required to route extra clocks signals. Going to a $5 \mathrm{X}$ implementation, this might not be feasible for very high data rates. For the ET-DR, if copies of data have to be routed to multiple lanes across the chip, then the performance at the leading end and trailing end would be dependent on the interconnect bandwidth with the attendant ISI and distortion problems. For clock signals, this is a more manageable problem.

Essentially, the MRCP-DR architecture has adopted the concept of three clock phases from the VI-3XO CDR architecture minus their dynamically adjustable nature. It has adopted the rotation concept from the ET-DR to track the data while providing a stable data detection window by exploiting available oscillator phases. Additionally, the MRCPDR architecture becomes an all-digital architecture that can be implemented using a digital design flow as an ASIC or onto an FPGA. Thus the MRCP-DR architecture is a hybrid of the VI-3XO CDR and the ET-DR architecture that can be implemented on an FPGA for prototyping. Custom implementations can also be pursued. This novel DR architecture is one of the contribution of this dissertation. The digital CDR architectures were reviewed in depth in Chapter 3 to highlight this connection to the reader.

The ET-DR architecture uses $\delta=90^{\circ}$, whereas $\delta=120^{\circ}$ would have been optimum for a $3 \mathrm{XO}$ CDR. The addition of the ninth phase would make this architecture closer to ideal, but the generation of eight phases is more practical and a differential QVCO can be used to generate the required eight phases with minimum innovation.

\subsection{Concept verification}

The MRCP concept has been verified using the flow shown in Figure 5.6. For initial verification, Simulink [17][18] was chosen because it has a comprehensive repertoire of readily available inputs, outputs and processing blocks. This was later replaced by Ver- 
ilog-AMS based verification [19][20] for a more consistent IC design flow. A custom ASIC implementation was attempted in parallel with an FPGA implementation. Due to the AMS multi-phase oscillator, a digital flow could not be employed.

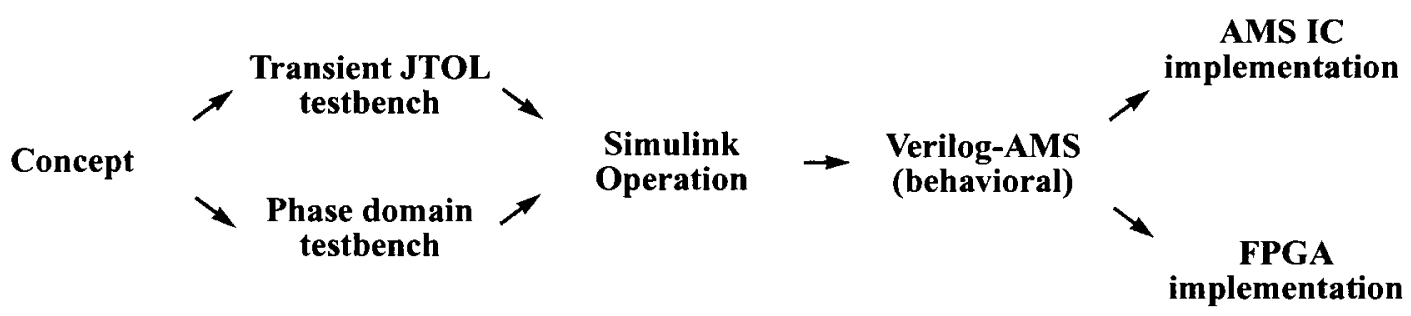

Figure 5.6 Verification flow

The preliminary simulations done for verifying the tracking performance using Matlab on the ET-DR and MRCP-DR architecture are shown in Figure 5.7. The traces are self-explanatory but are discussed in more detail later in conjunction with verilog-A simulations. In Figure 5.7(b), the central clock phase (CKD_CENTER) establishes the sampling point. In order to provide a stable detection window, two additional phases are tapped and rotated in tandem with the center phase-early (CKD_EARLY) and late (CKD_LATE). Thus a 3XO DR architecture is established.

By transferring the burden of maintaining phase distances to the wideband PLL, three things happen. First, the tuning range of the multi-phase oscillators can be used to implement a multi-protocol data recovery using this strategy. Second, the inter-phase spacings are PVT-invariant and maintain nominal spacing. Third, JTOL does not fluctuate with the fluctuating PD characteristics. The clock generator module is common for several lanes on-chip and further re-use of hardware can be made by implementing capable clock 
buffers. For a $3 \mathrm{XO}$ PD, a multiphase oscillator in a wideband PLL configuration and a technology with a high-enough $f_{\tau}$ are a prerequisite for a custom implementation.

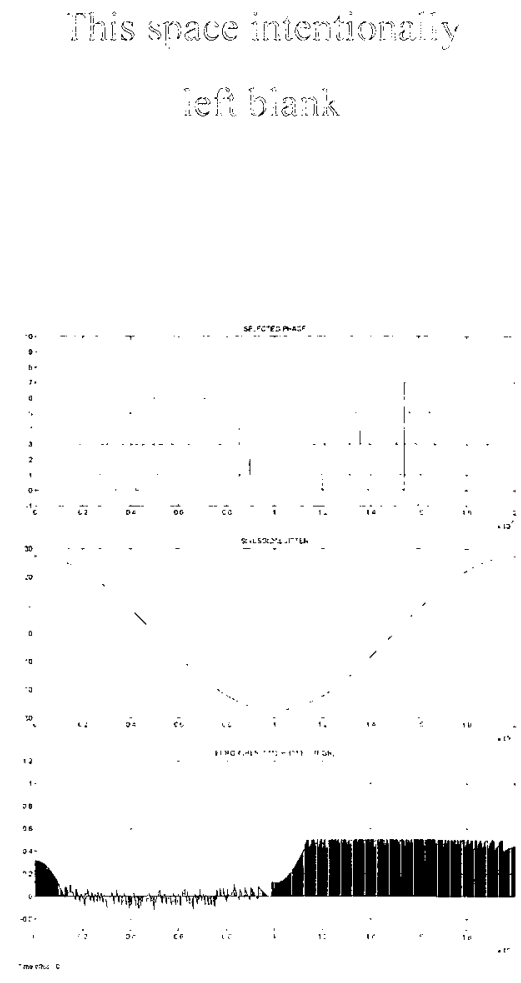

(a) ET-DR operation
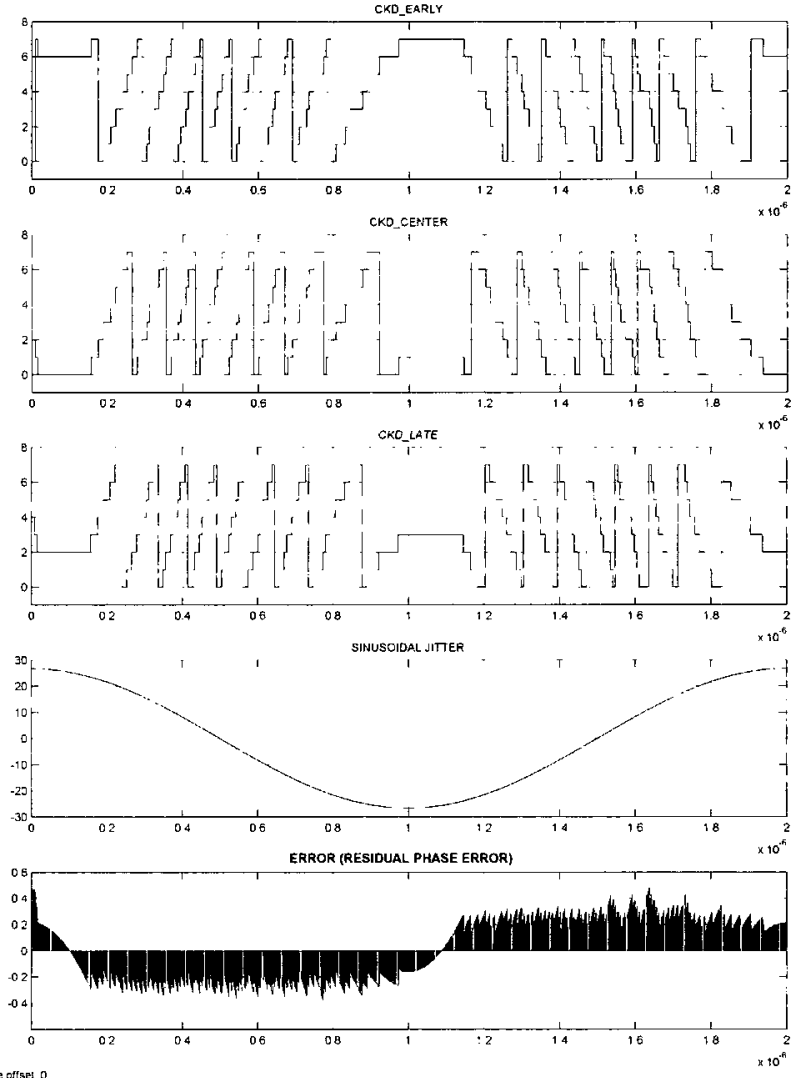

(a) MRCP-DR operation

Figure 5.7 Comparing the tracking performance using Matlab

\subsection{Simulating jitter tolerance}

A major bottleneck in the design of CDR systems is the efficient evaluation of its performance at the conceptual or design stage. This is made complicated due to the indirect consequence of Amdahl's law. A serial transient simulation cannot be run any faster than the time required to solve the circuit's Jacobian matrix at every successive time step. For a serial link, the time constants associated with the pattern length, jitter frequencies, modulation and bit rates vary over several orders of magnitude. CDR simulation is further complicated by the fact that BER of $10^{-12}$ is considered acceptable for most HSSL sys- 
tems. To encode transitions, the Nyquist frequency criterion applies. At high frequencies approaching the limit of the technology, where the rise time and fall time and on time are roughly equally divided, it would mean three samples per UI and hence a total of $3 \times 10^{12}$ samples per set of billion bits. For statistical confidence in the error results, this is to be repeated many times over [155]. Hence, it is impractical to undertake transient simulations as the only vehicle of verification over all modulation ranges. A combination of strategies is required. Transient JTOL simulations are to be run, however, at all stages of chip design to detect any functional shortcomings in the design due to unmodeled phenomena.

The future of a brute-force transient bit simulation is bleak and is unlikely to improve in coming years. The single core performance of CPUs has not improved significantly since 2004 [143] due to the demise of Moore's law and is unlikely to improve due to packaging and thermal considerations in CPUs. Parallel simulations performed on multi-core CPUs help in expediting parametric simulations, but the situation for a single transient simulation does not improve significantly.

\subsubsection{Phase domain jitter tolerance testbench}

In the phase domain, the performance of the CDR can be verified in manageable time for the requisite BER performance and the previous chapter has shown such results in Figure 4.21 and Figure 4.22. The phase-domain simulation methodology is clearly explained in the literature [35]. Setting up the proper phase domain model for a novel architecture is quite challenging. Cross-correlation with a synthesized FPGA version is equally difficult during prototype testing. 


\subsubsection{Behavioral jitter tolerance testbench}

The first step in the functional verification of a DR circuit is the set up of a JTOL testbench. The details for this JTOL setup are shown in Figure 5.8 as they have appeared in prior publications [19][20].

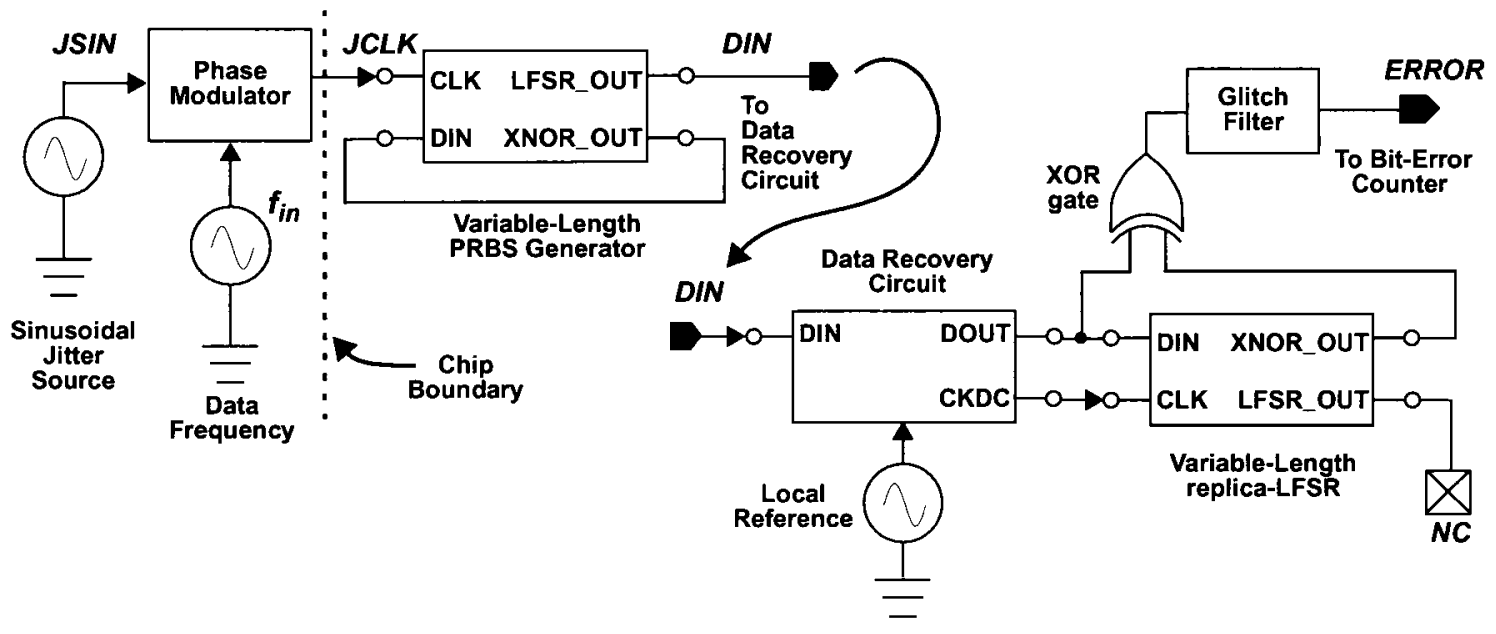

Figure 5.8 JTOL testbench

The testbench operates by modulating a clock source with a sinusoidal jitter (JSIN signal) that results in a jittered clock (JCLK). The JCLK signal drives a variable-length PRBS generator that produces the jittered data (DIN). The signal DIN travels through the channel and an equalizer (not shown) and reaches the data recovery circuit that is the device under test (DUT). After the data is recovered at the output (DOUT) terminal of the DR circuit, the recovered clock (CKDC) is now used to drive the replica LFSR that has same number of flipflops as the input PRBS generator. As correlation is established between the two LFSRs, the error (ERROR) pulses disappear. The ERROR signal can 
drive a counter to count the number of errors during debugging. Figure 5.9 shows a typical

JTOL transient simulation and explains important events on the simulation.

(A) Jitter reaches its peak

(B) Maximum change of jitter; Tracking is limited by the phase step and update period.

(C) Tracking jitter

(D) Tracking direction has reversed; Notice phase wrapping happens three times as DJ=3 UI-pp

(E) No tracking required at the point corresponding to(A)

Errors are likely to happen near (B) and the slope of the sinusoid is determined by its amplitude

(F) Notice the constant phase difference between the 3 of 8 phases

(G) The multiphase DLL is given an initial condition to expedite locking

Full Simulation 3.Ø UI-pP @ $1 \mathrm{MHz}$ (With DLL)
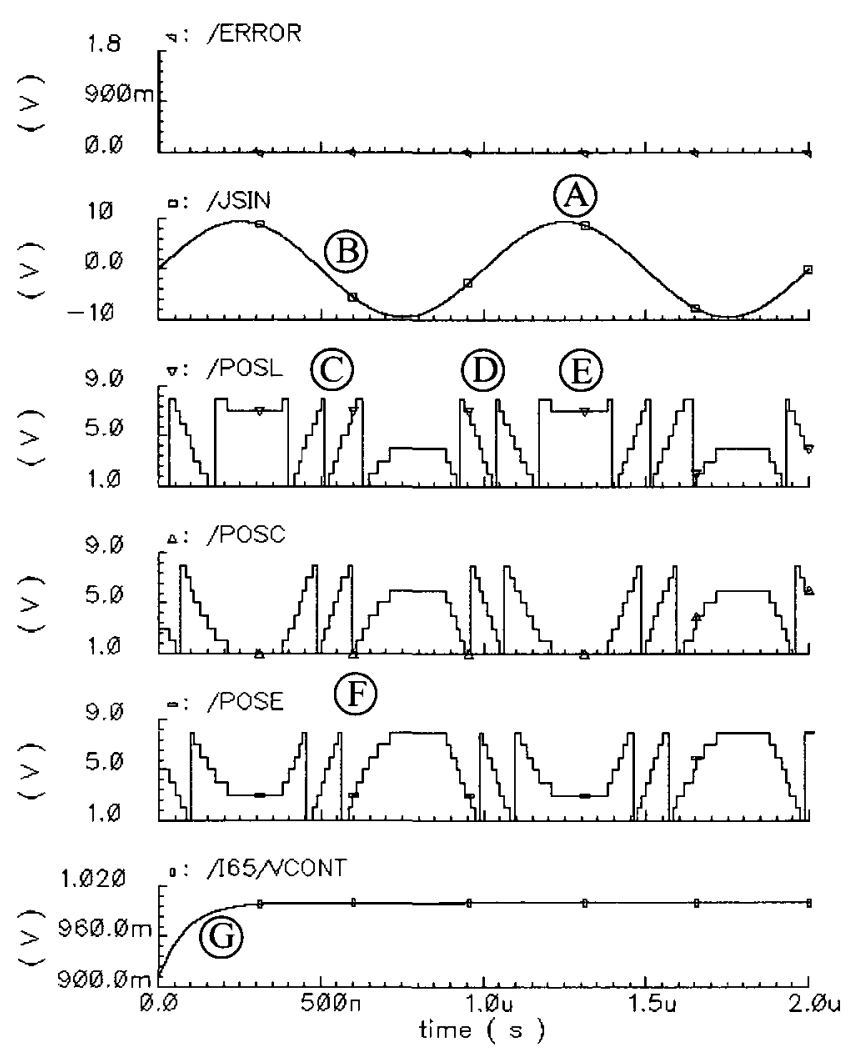

Figure 5.9 Functional verification of JTOL using Verilog-A

Initial conditions are set on the $V_{\text {cont }}$ line to achieve faster lock and the ERROR signal is monitored for errors. The POSL, POSC and POSE are the positions of the late, center and early clock phases respectively. Since there are eight phases, the values wrap around from 8 to 1 and vice versa as the JSIN signal changes direction. Appropriate pulse width filtering is employed to eliminate false ERROR pulses.

Similar methodology is used in BERT instrumentation. If one erroneous bit enters the replica LFSR, it generates a number of errors depending on the feedback connections of the LFSR until the errors are flushed out at the other end and correlation is re-estab- 
lished. Commercial BERTs compensate for this in order to detect the true error count. Such functionality is not pursued in this simulation.

\subsubsection{The basic idea of a jitter tolerance simulation}

The basic idea is that the frequency and the amplitude of the JSIN signal are changed in a systematic manner. This generates a 2D space that is swept. If the ERROR pulses appear after the system has settled, then the particular point in the $2 \mathrm{D}$ space fails the JTOL test. The number of errors is important for commercial BERT systems since RJ is also added and a proper BER characterization is required. Adding RJ in a transient simulation is not feasible. The advantage that the BERT instrument has is that it is running at speed, whereas the simulations run extremely slow.

\subsubsection{Strategies to reduce JTOL transient simulation time}

If the phase step that compensates for the jitter is not large enough or the sampling phase updates are not frequent enough, then the jitter cannot be tracked from cycle-tocycle resulting in transient bit errors. This effect is felt the most at the zero crossing where the slope of the JSIN signal is at its maximum. Away from the zero crossnig of the JSIN signal, the CDR circuit is able to cruise comfortably. It is, therefore, not useful to use the whole JSIN signal for simulation purposes. The slope of the JSIN signal can be manipulated in a way that stresses the CDR circuit to cause errors.

\subsubsection{FM and AM chirps}

The JSIN signal neither has to be at the same frequency nor the same amplitude for the duration of the simulation as shown previously in Figure 5.9. The amplitude and frequency of the JSIN signal trace a path in the 2D JTOL plane vertically and horizontally, respectively. The results in Figure 5.10 elucidate this concept. When the amplitude or the frequency of the JSIN signal increase beyond the CDR circuit tracking range, bit errors occur rapidly. The range of the applied AM and FM chirps [19][21] can be managed intel- 
ligently to determine the JTOL for a particular CDR circuit. There is no particular reason to label these chirp signals as the JSIN signal other than the fact that several signals are being multiplexed in the same testbench and the node has not been renamed to avoid confusion.
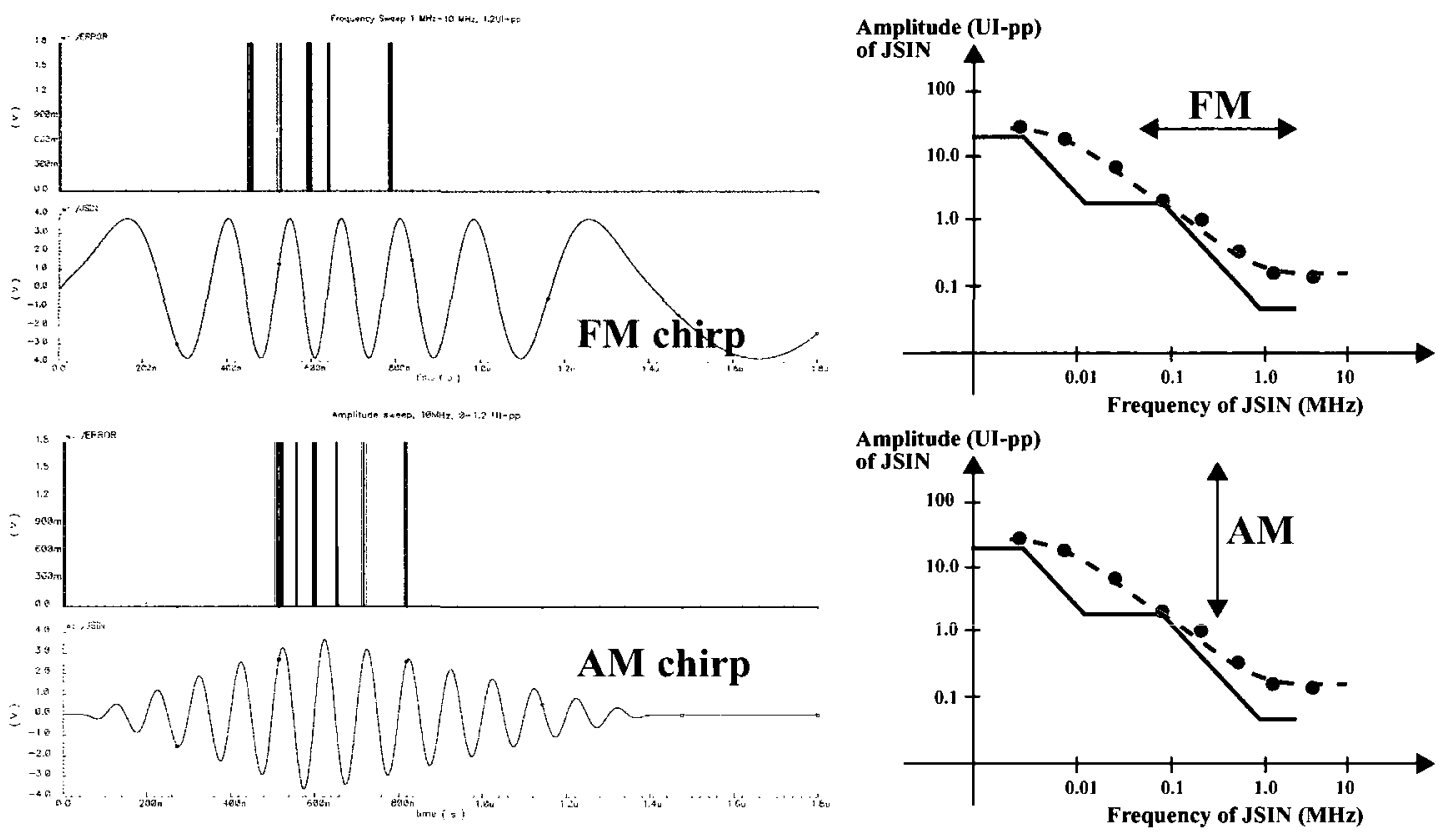

Figure 5.10 AM and FM chirps on the JSIN signal

\subsubsection{Ramp input as the JSIN signal}

Since it is the slope of the jitter signal that causes bit errors, a ramp with the same maximum slope can be used instead - but with some caution. For the AM and FM chirps, a shorter simulation using a ramp signal can find the exact amount of slope that can be tolerated. This reduces the simulation time to a manageable level without sacrificing the accuracy of the transient simulation [21]. A typical result appears in Figure 5.11. The phase model simulations are good for the architectural stage but are not feasible prior to tapeout where the functionality and performance need to be ascertained. There might not be a one-to-one correspondence between the phase model and the actual circuit since models are never complete. The reduction in simulation time using a ramp signal requires a simpler test input. The designer must wait for the initial transients to settle. Once again, 
the ramp input retains the JSIN label. The simulation time can be shortened by using a ramp signal and the simulations can be parallelized and/or parametrized for maximum efficiency.

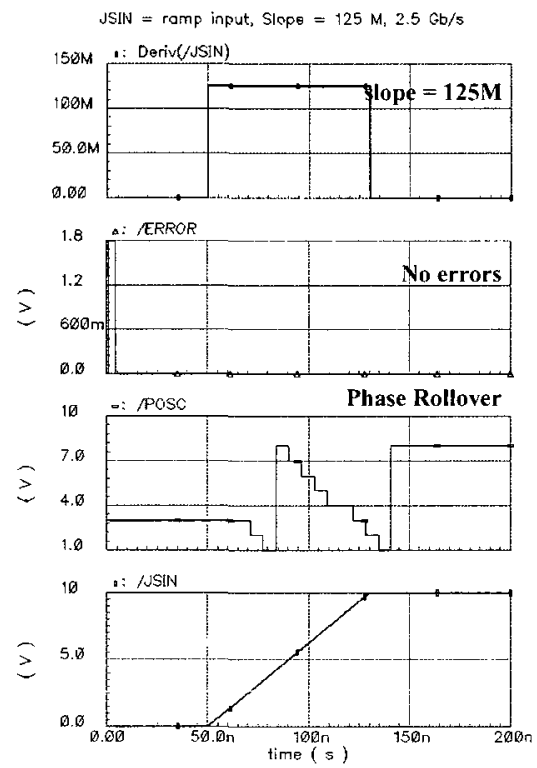

(a) No errors at slope $=125 \mathrm{M}$

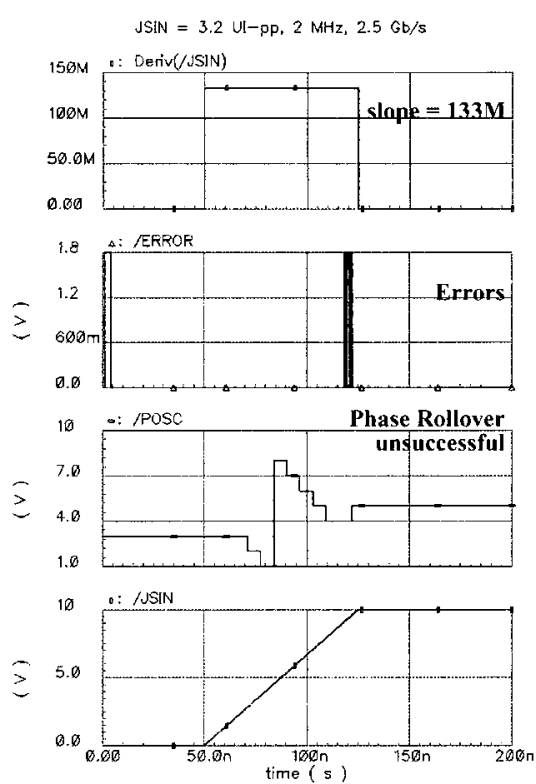

(b) Errors at slope $=133 \mathrm{M}$

Figure 5.11 Ramp input as the JSIN signal

With the above-mentioned techniques, it is possible to accurately characterize a CDR system without loss of accuracy. For simulating low frequency wander with the low time constants involved, the ramp method is more suitable. As a final note, the simulation results from the phase-domain model can be mixed with the HF JTOL results from the accurate time-domain simulations to get a clearer picture of the CDR performance.

\subsection{Representative simulations for the MRCP-DR}

It can be observed in Figure 5.12 (a) that the CDR bandwidth suffers as a result of reducing the bit rate. This occurs because the number of edges per unit time reduce. Having the same number of oscillator phases at a lower bit rate is beneficial, however, and it improves the HF JTOL as the UI widens and thus becomes more tolerant to the same DJ. 
In order to achieve the same bandwidth at the half the bit rate, the update period is halved and the CDR performance returns to its original level.

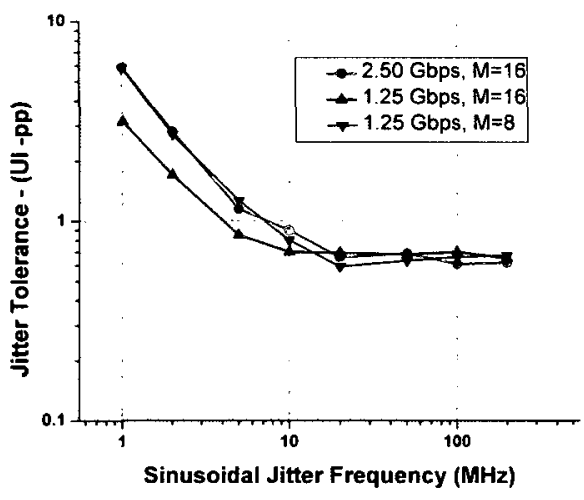

(a) Different bit rates and update frequencies

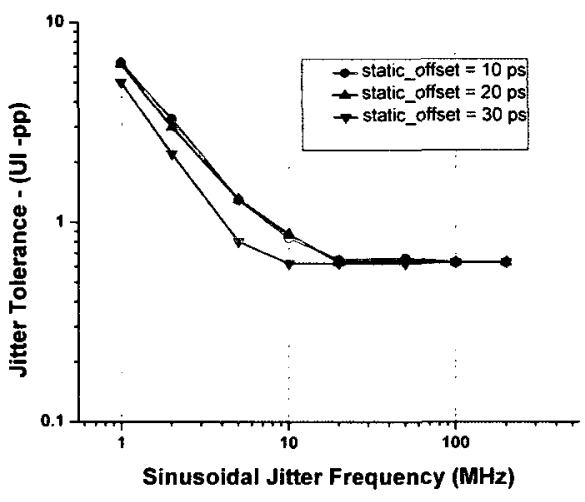

(b) Static offsets in inter-phase spacing

Figure 5.12 Jitter tolerance - MRCP-DR architecture

In Figure 5.12(b), the effect of a static phase offset in one of the DLL phases is shown. For a non-rotating oversampling CDR system, this would have been a more complex phenomenon depending on which phase was aberrant. If the aberrant phase were near the locking point, it would modulate the CDR bandwidth and accurate performance could be found using the software introduced earlier. For a rotating NXO CDR system, however, the phases do not have a static identity and the static offset is dithered out due to the rotation. Beyond 0.125 UI, severe degradation of JTOL occurs due to static phase offsets.

In order to further study the robustness of the DR circuit, more simulations are performed for the frequency offsets in the oscillator. The JTOL performance for an offset of $10 \mathrm{MHz}$ is shown in Figure 5.13 (a). For a locked oscillator, this is not an issue, but for a free-running oscillator, this puts an upper limit on the the JTOL performance. Frequency tolerant CDR circuits have been discussed in the literature [94].

The effect of PRBS length can be seen in Figure 5.13(b). With a longer PRBS, the bandwidth and the HF JTOL suffer due to the CID run length. The degradation gets espe- 
cially pronounced if the longest CID run and the update period are roughly the same and occur at the most inconvenient time.

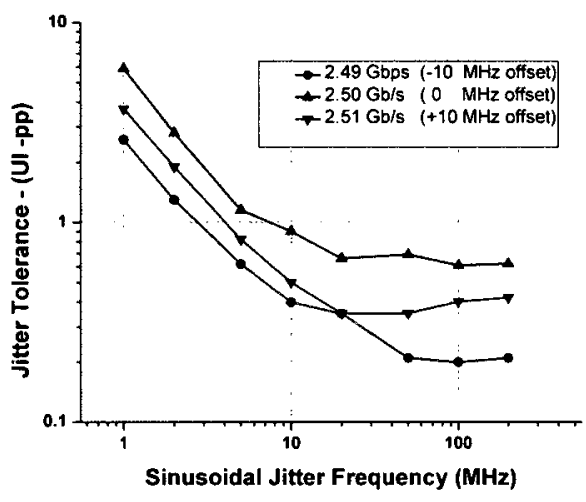

(a) With frequency offsets

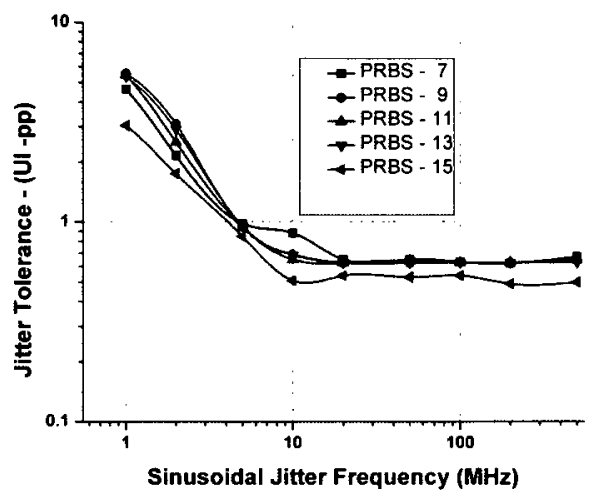

(b) Using a variable length PRBS

Figure 5.13 Jitter tolerance - MRCP-DR (continued)

Finally, the tracking performance of the ET-DR architecture and the MRCP-DR architecture are similar as shown in Figure 5.14, only robustness and the digital implementation aspects are improved.

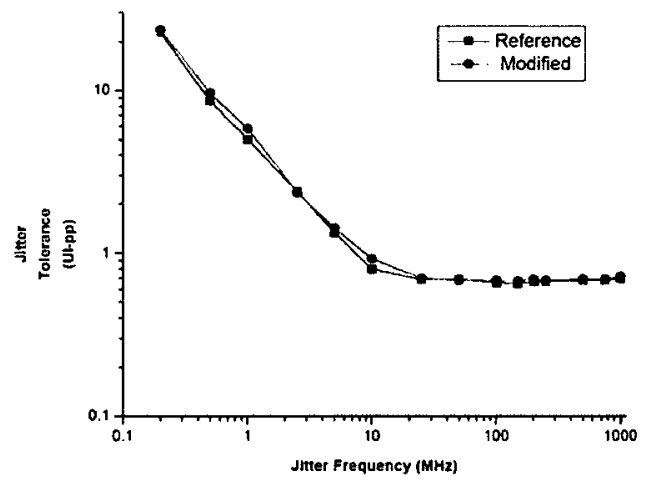

Figure 5.14 Comparison of tracking performance

\subsection{Implementation}

The MRCP-DR architecture has been implemented in an IBM process (CMRF8SF) on a 0.13 um process node. Although the measurements from the fabricated 
chip were not repeatable and therefore inconclusive, some estimates can still be made for future work. Figure 5.15 shows the chip layout with various modules marked.

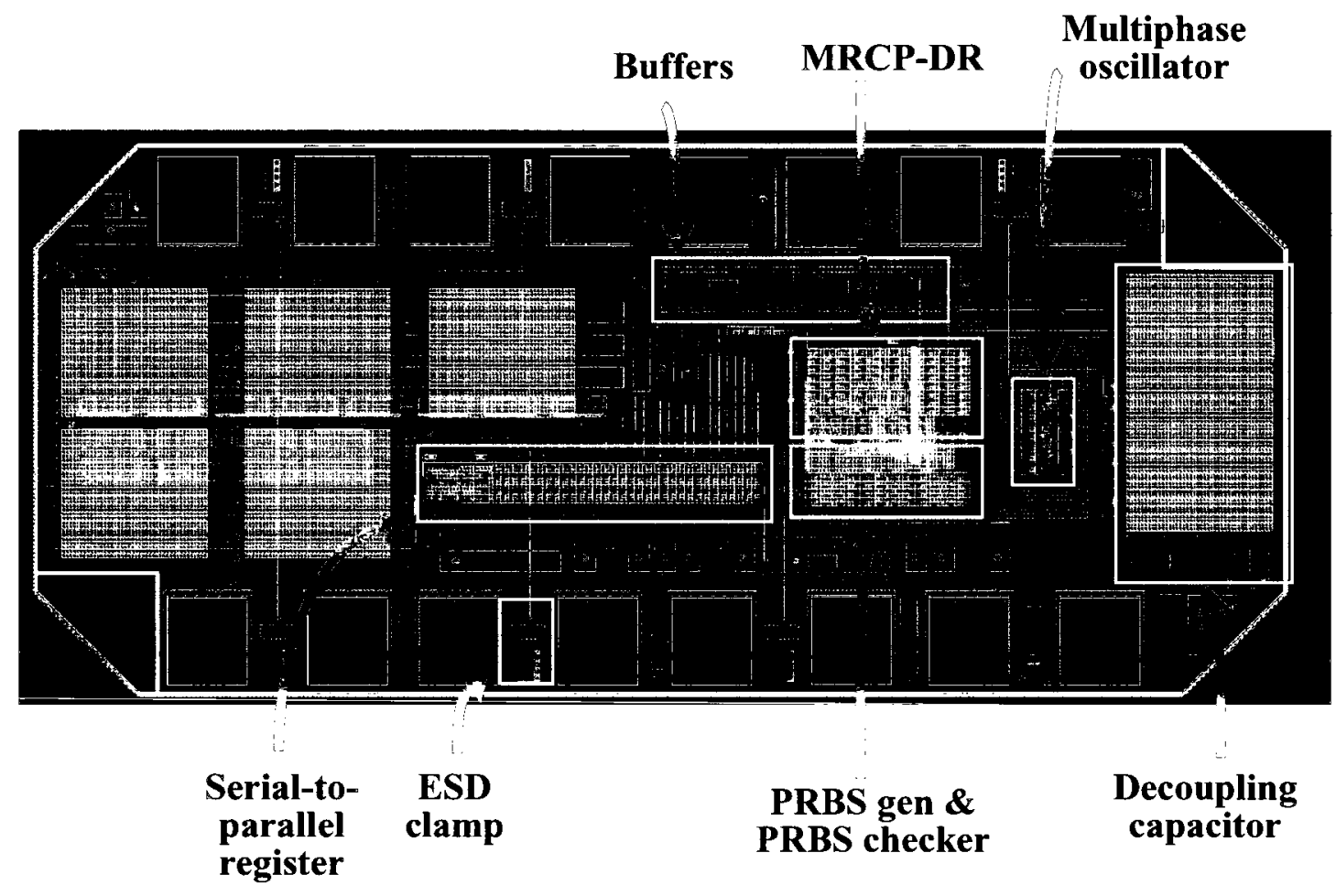

Figure 5.15 Chip layout

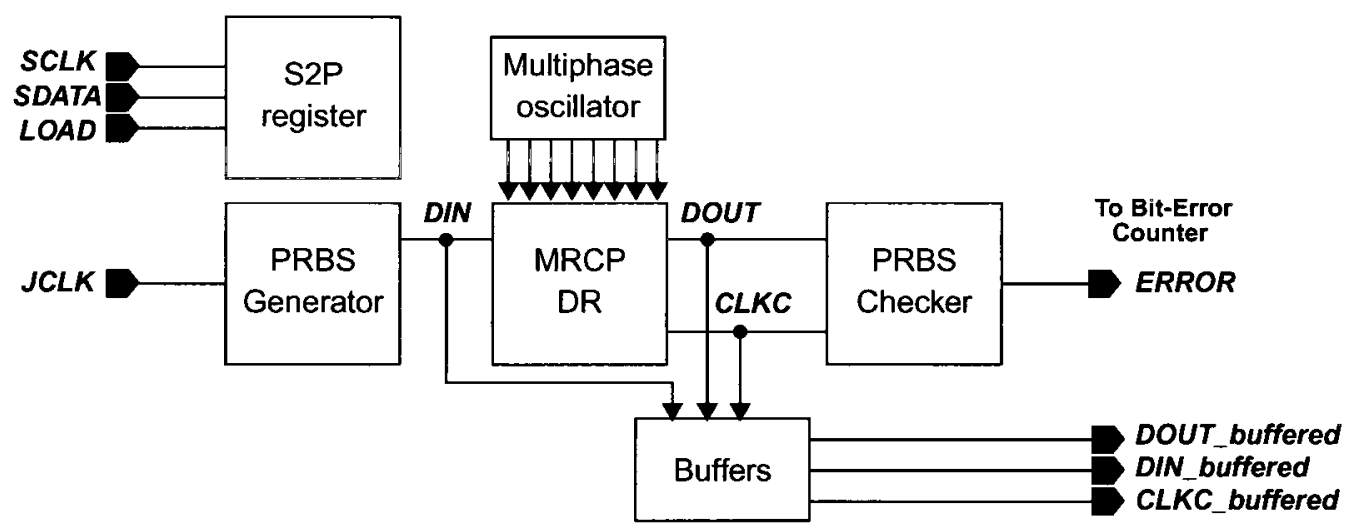

Figure 5.16 Top-level chip block diagram

Figure 5.17 shows the die photograph of the chip. The vias along the outside and the fill in many layers is done at the fabrication facility. The effect of the fill on oscillator performance can be detrimental and needs to be simulated, if possible. Due to shortage of 
probe pads, options were built into the chip for the second run. The implementation was pad limited and thus surgery was required on the second version. The second chip was dead-on-arrival so surgery was not very helpful
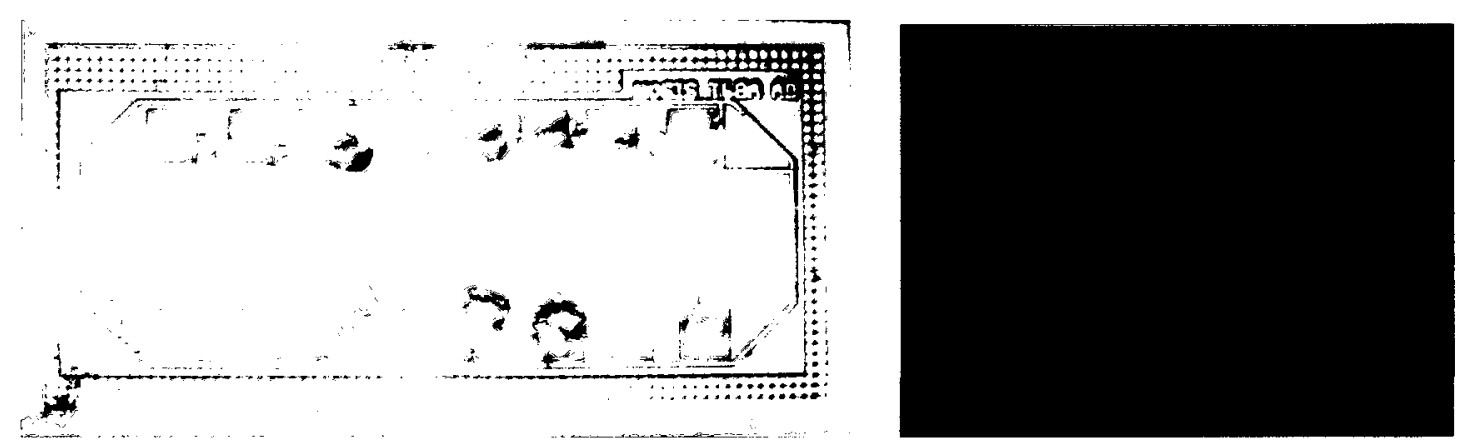

Figure 5.17 Die photograph and laser surgery close-up

The osciliator was designed for $1.25 \mathrm{GHz}$ perîrmance with 8 phases but performed at $1.05 \mathrm{GHz}$ (maximum) perhaps due to the filling. Partial and inconclusive results are shown in Figure 5.18 for $500 \mathrm{MHz}$ DR operation. The rented sampling scope had only one sampling head so the side-by-side display of clock and data was not possible. A simpler scope was used for that purpose to verify functionality. The trigger was set to the CKDC signal, and that dithered the CKDC signal around so it is not a convincing picture. The spectrum was also found by disconnecting the scope in a different setup so the frequency is slightly different. A probe station is not suitable venue for changing instruments frequently.
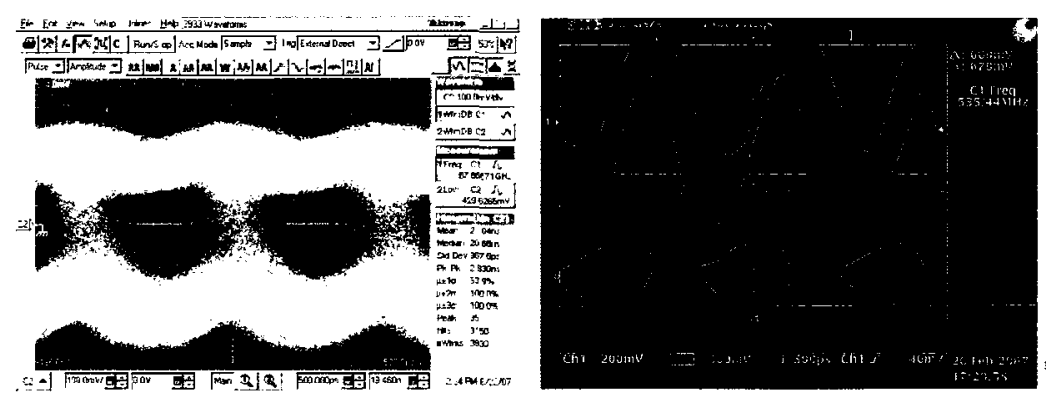

(a) DR operation @ 500MHz

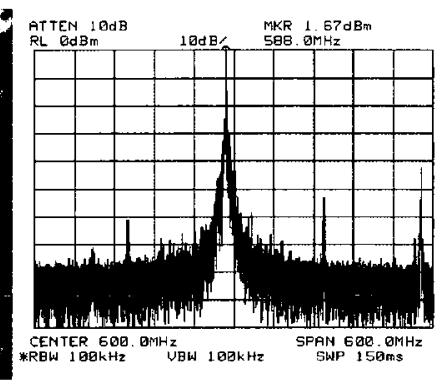

(b) CKDC spectrum

Figure 5.18 Partial measured results for the custom chip 
Due to the shortage of input pins, the initialization commands were sent through the serial interface to the registers. The oscillator, DR circuit, serial-to parallel register and the PRBS arrangement were all untested in real silicon so the success was elusive.

\subsection{FPGA testbench}

Since the architecture is all-digital in nature, it can be implemented on an FPGA board at a reduced bit rate. The FPGA testbench is shown in Figure 5.19. The Altera-DE2 development board was used for this experiment. The PRBS generator and checker were implemented on the FPGA. An Agilent function generator was used to characterize the implementation at $50 \mathrm{MHz}$ and $25 \mathrm{MHz}$. These round numbers were chosen since the PLL macro on the FPGA worked reliably upto $450 \mathrm{MHz}$ and a 8-phase output is realized by dividing down using digital counters.

The FPGA set up shown in Figure 5.19 consists of an Agilent $3.0 \mathrm{GHz}$ signal generator that delivers a phase modulation capability upto $50 \mathrm{kHz}$, a bias-T, a power supply, an Altera-DE2 educational board and an Agilent $20 \mathrm{GHz}$ sampling scope along with a personal computer.

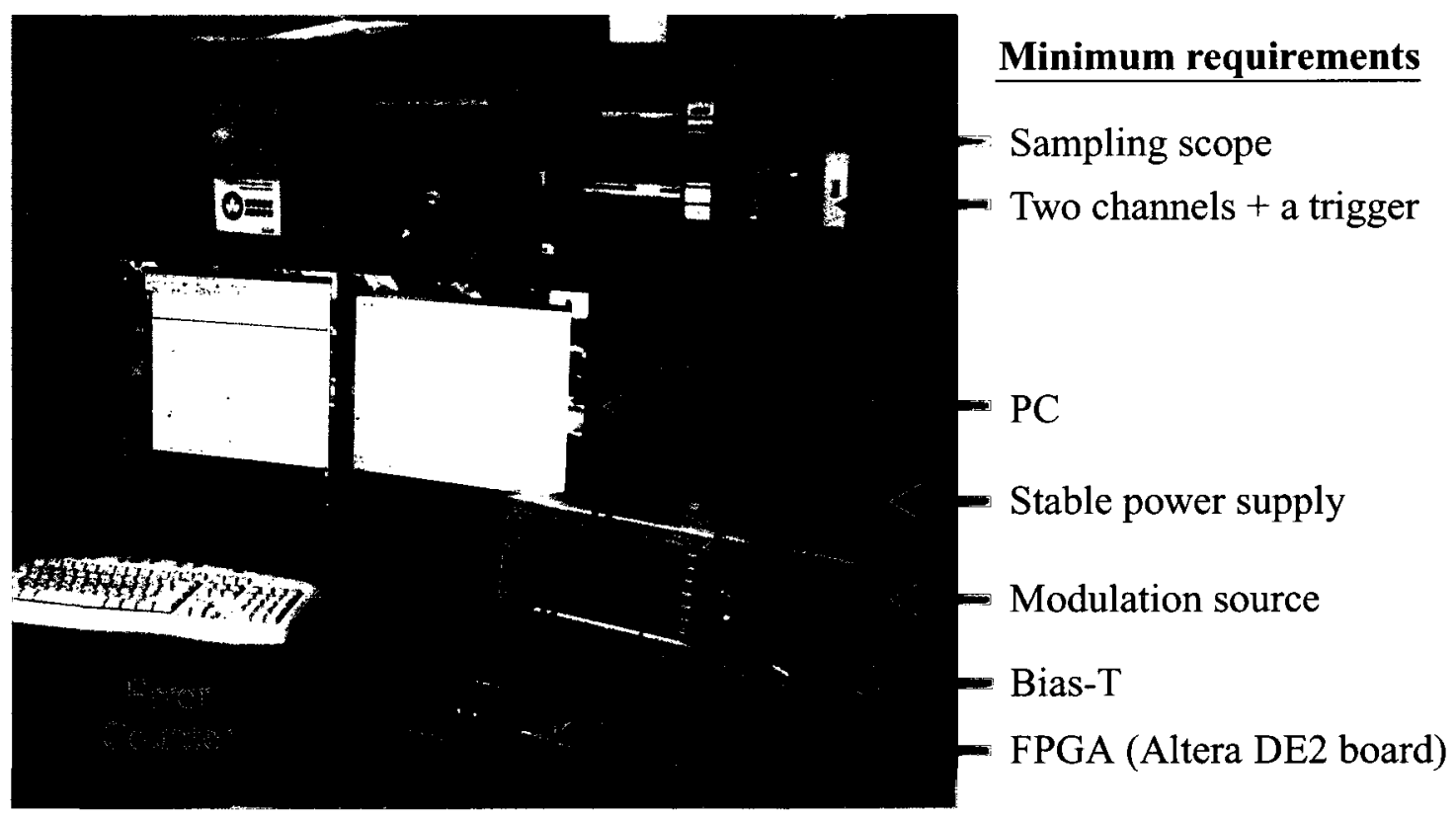

Figure 5.19 FPGA test setup 


\subsubsection{FPGA test results}

The DR circuit successfully recovers data from a PRBS at 50 Mbps, PM-modulated with 0.5 pi rads @ $2 \mathrm{kHz}$ as shown in Figure 5.20(a). The input is labeled as DIN and the eye is closed due to the modulation from the function generator as shown by the histogram. The recovered data is labeled as DOUT and is being recovered using the PLL macro on the FPGA that provides multiple clocks. A representative multi-phase generation scenario at $25 \mathrm{MHz}$ is shown in Figure 5.20(b).

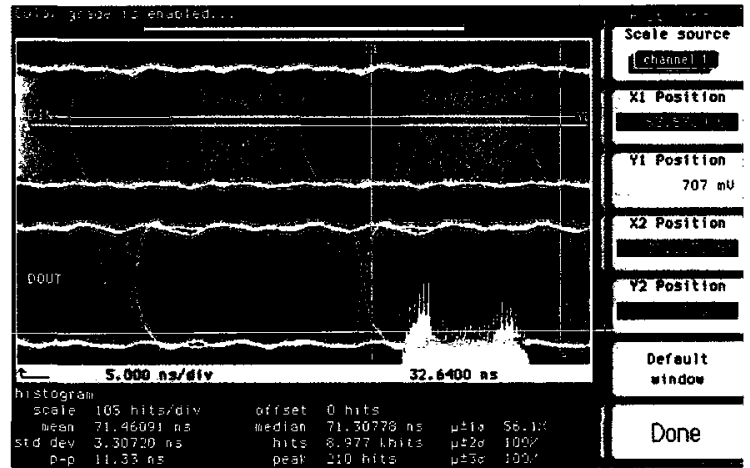

(a) Tracking PM@50 Mbps

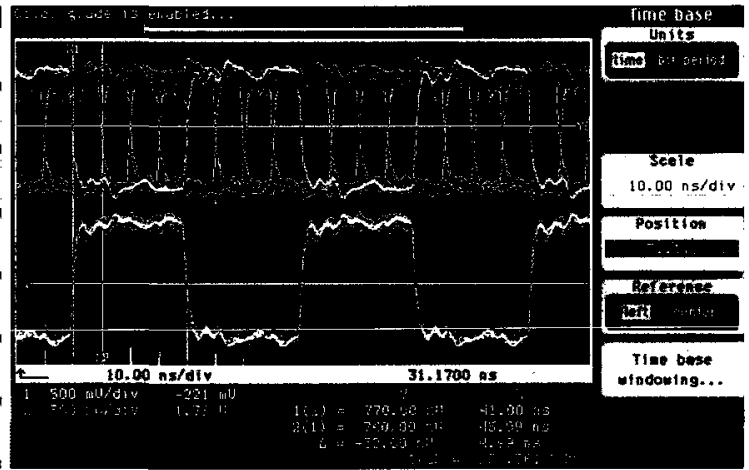

(b) Generation of 8 phases (25 MHz)

Figure 5.20 Tracking results and phase generation

Due to the lack of an instrument to generate RJ or the ability to mix it with DJ, a verilog module is implemented that selects an edge from a multitude of edges and randomizes the selection such that $\mathrm{RJ}$ can be produced. It is not robust to characterize $\mathrm{RJ}$ in this fashion on an FPGA platform since the edges that are being selected undergo different delays due to reflections through the synthesized chain and are not precisely known due to platform restrictions. As such, increasing the delay to the entire UI is not reliable and the strategy breaks down much earlier. The results for this RJ generation and operation are depicted in Figure 5.21. Even if it is possible to come up with another RJ generation scheme, it is not a standardized method and hence the results cannot be compared with an existing result. Characterization is limited to the modulation range provide by the Agilent signal generator and a $20 \mathrm{~dB} /$ decade slope is seen in the JTOL characteristics. The reader 
can notice the shape of the histogram in Figure 5.21 and contrast it with the one shown in Figure 5.20. It is to be noted that the DR circuit keeps working with the RJ applied.

Proper characterization will be reported along with synthesis details in a later publication, pending the availability of proper instrumentation. The fact that it can be implemented on an FPGA is confirmation of its all-digital status and nothing more can be emphasized here without further experimentation.
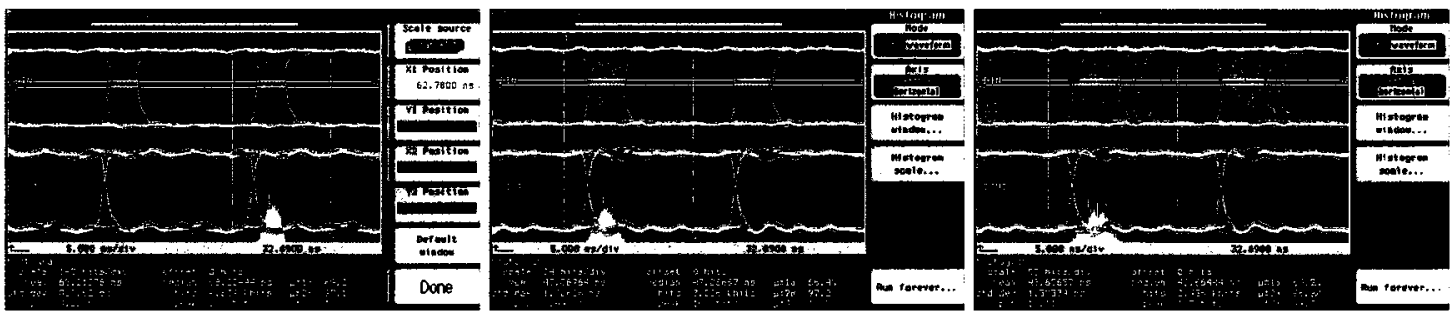

\section{RJ spread increasing}

Figure 5.21 Introducing RJ using random edge selection

\subsection{Summary}

This chapter explained the rationale behind the novel multiple-rotating-clockphases DR architecture. Representative simulations and comparisons with existing architectures were discussed. The results from a custom ASIC implementation were presented along with more successful ones from an FPGA implementation. Efficient and novel techniques for simulating jitter tolerance were also proposed. The all-digital DR architecture and the novel JTOL simulation techniques are contributions of this dissertation. 
"The press, the machine, the railway, the telegraph are premises whose thousand-year conclusion no one has yet dared to draw"

Friedrich Nietzsche

\subsection{Chapter overview}

This chapter reviews the main points in the dissertation. The contributions from this dissertation are listed thereafter.

\subsection{Review of the main points}

- The incoming data acquires jitter from the channel that is not under the control of the SerDes designer and hence the whole point of developing a SerDes IP.

- CDR and DFE units are essential blocks in a SerDes system. Their design is interdependent. DFE opens the vertical eye opening and the CDR circuit opens the horizontal eye opening.

- SerDes usage and intellectual-property integration in an existing product requires intensive early-stage planning. An understanding of the trade-offs allows for choosing better design flows and architectures. First-pass success requires efficient simulation, usually performed in the behavioral domain.

- A bang-bang phase detector (BBPD) provides an early or late indication of phase only. Every data edge is a frequency acquisition problem for the BBPD. A frequency step - upor down-is applied in the locked state to the VCO in order to implement a BBPD-based CDR circuit. 
- At the other extreme, a linear phase detector (PD) is combination of an infinite numbers of BBPDs with varying thresholds, each contributing a small amount toward the final output. A PD with an infinite oversampling ratio $(O S R)$, i.e., a linear PD cannot possibly be justified, even theoretically.

- In practice, a linear PD is not realizable due to analog design constraints that have been discussed in the dissertation. The mathematical analysis is straight-forward since the PD characteristics remain unchanged in the presence of incoming jitter.

- In practice, a BBPD can be realized as long as a flipflop can be realized in a given process node. A digital architecture can be realized with fewer resources and ports well compared to a linear architecture. The mathematical analysis of a BBPD, however, is cumbersome since any component that deciphers the sign of the input is dependent on the statistics of the input signal.

- Hence the goal is to have a manageable mathematical framework, and the combination of multiple BBPDs in order to realize the advantages of linear analysis along with a digital CDR implementation. Multiple BBPDs with different decision thresholds can thus be combined to realize oversampling PD architectures. The incoming data is sampled at multiple points during the UI.

- There are two methods to generate multiple phases. Delay elements are a bruteforce method and suffer from PVT-induced variations-although very promising time-to-digital architectures have been reported. In a PLL configuration, the interphase spacing of multiple oscillator phases is largely immune to PVT variations.

- In order to combine theory with practice, the quest is to find the minimum $O S R$ and adequate inter-phase spacing $(\delta)$ to achieve the specified BER, given the type and quantity of input jitter.

- An interactive software has been developed to study the time-averaged behavior of PDs in the presence of input jitter containing RJ, DJ and asymmetry.

- The interactive software uses a generalized analysis to combine concepts as varied as the BBPD, oversampling PDs and the linear PD using one coherent methodol- 
ogy. This is made possible by the advanced mathematical and symbolic analysis capabilities of Mathematica ${ }^{\circledR}$, that is a commercially available tool.

- The $n-x$ oversampling (NXO) PD characteristics - that have been parametrized for $O S R$ and the interphase spacing $(\delta)$-are convolved with the incoming jitterthat has been parametrized to include DJ, RJ and asymmetry. The resulting equation for the time-averaged NXO PD behavior is differentiated to find the NXO PD gain in analytical form. This information is plotted using the dynamic and interactive functionality of Mathematica $^{\circledR}$. The resulting equations have several parts that do not advance human understanding per se and would be quite cumbersome to manipulate manually. Visually and dynamically, however, it is easy to see when the averaged PD output is acting almost like that of a linear PD such that a further increase in the $O S R$ provides only marginal benefit.

- An increase in the $O S R$ requires more oscillator phases thus necessitating more power and design time, so it is to be generally discouraged. More clock phases reduce the phase jump at the output improving the jitter transfer specification.

- Essentially, the time-averaged mathematical behavior of all PDs follows a simialr theme. There are just more $\tanh ($.$) terms in the final equation for the case of larger$ OSR. In this respect, the linear PD output is nothing but a combination of infinite number of $\tanh ($.) outputs with varying parameters. Analytically, linear PD analysis is a special case of BBPD analysis. Oversampling PDs are simply stepping stones toward the linear case. Infinite steps cannot be required in any real system.

- The understanding of $O S R$ on CDR performance alone is not useful for design purposes. In this respect, the advantage over prior art is the availability of parametrized and exact equations for time-averaged NXO PD characteristics. Using these equations, a closed-loop simulation flow is developed that runs the ultra-efficient phase domain JTOL and JTRAN simulations. Many architectural decisions can be answered at this early stage using this simulation flow. The $O S R$ and the interphase spacing can be tweaked for the required bandwidth and tracking performance.

- To translate erf(.) function into $\tanh ($.$) , use \operatorname{erf}(x)=\tanh (1.2028 x)$. 
- The inclusion of a proper statistics toolbox and equation-solving functionality in behavioral-AMS simulators is a logical next step in their evolution.

In conjunction with the implementation of a novel all-digital multiple-rotatingclock-phase (MRCP)-data recovery (DR) architecture, the important points are as follows:

- The MRCP-DR architecture counters the static phase offset problem by tapping available phases from a multi-phase oscillator. This guarantees PVT invariance for establishing the inter-phase spacing. Delaying the data twice using delay elements introduces more jitter variance. Using multiple clock phases on the same copy of the data results in the introduction of no additional jitter on the data.

- Due to rotating multiple clock phases, the effect of static oscillator phase offsets on DR performance is reduced. However, it still needs to be simulated.

- A novel all-digital MRCP-DR is implemented using Verilog-HDL-driven FPGA prototyping. It uses an on-FPGA synthesizable PLL macro that is used to generate multiple clock phases.

As far as efficient simulation of a CDR system is concerned, the important points are summarized below:

- SerDes systems cannot be simulated end-to-end using transient circuit simulations due to their size and the range of time constants involved.

- Behavioral modeling using a circuit-aware AMS language is crucial for running complex simulations on large systems. Jitter tolerance (JTOL) and jitter transfer (JTRAN) simulations are perfect examples of complex simulations.

- Transient JTOL simulations entail no loss of accuracy but since these simulations are time-intensive and not practical, a succession of novel strategies was introduced in this work to drastically reduce simulation times.

- The transient bit errors happen at the point of the maximum slope of the jitter causing sinusoid. If the slope of the sinusoid is the reason for errors, then the whole sinusoid does not provide much extra information. 
- The novel technique, therefore, modulates the jitter sinusoid with respect to its frequency and amplitude. This causes the slope of the jitter sinusoid to change.

- Using these frequency and amplitude chirps, the 2D plane for JTOL is swept in horizontal and/or vertical directions until the onset of transient bit errors. The sweep range is gradually reduced and the compliant values are recorded.

- For simulating low frequency wander, the novel AM and FM chirp methodology is refined and a ramp with an equivalent slope is substituted to realize further reduction in JTOL simulation time.

- A combination of these slope-exploiting techniques can result in a transient characterization of the system without loss of accuracy.

- The results from HF JTOL transient simulation and phase-domain JTOL simulation for wander can be combined for achieving optimum simulation times as the design of the chip proceeds from the exploration stage to the actual design stage.

- SerDes debugging is a many-to-one challenge. A multitude of sources can cause the same effect at the output-eye closure and BER degradation. Human intuition is not very helpful during this characterization. Specialized instruments to measure jitter go a long way in detecting and eliminating the characterization bottlenecks. Simulation can also benefit from statistical, phase-domain and behavioral methods and remains an area of active research.

- If the focus of the CDR operation switches from clock-centered to data-centered recovery, then more resources are needed to accomplish the same goals, since data changes every clock cycle and must be stored. Therefore, blind oversampling used for data recovery is a wasteful technique but oversampling itself is not. The information about the clock comes from an oscillator and hence requires much less effort to store, save, and control due to the nature of the oscillator in feedback systems. As opposed to this, saving the changing data in a memory (or a FIFO) clocking at speed is wasteful in terms of area and power. 
- The FPGA offerings as of 2010 are sorely missing a very useful block-the DCO. Another less useful but equally important missing block is a current output thermometer DAC. These components bridge the world of the digital and the analog.

The research into CDR systems is by no means over. However emergent and dominant trends were summarized at the end of Chapter 3 and will not be repeated here. It makes commercial sense to combine several lower frequency CDR IPs using a MUX/ DEMUX arrangement instead of using brute-force to accomplish higher data rates. This is the direction that the industry has adopted. The focus on the $40 \mathrm{Gbps}$ CDR lane is not very productive until testing equipment, simulators and transistor models can catch up. Like the CPUs before them, the SerDes macros are maturing in terms of techniques and established practices. The success metrics of the future are compatibility between vendors, self-testing, redundancy, lower power, reliability, reduced time-to-market and re-configurability for a multi-protocol SerDes paradigm.

\subsection{Contributions}

This work has resulted in the following contributions:

- For CDR operation, an optimum oversampling ratio $(O S R)$ and inter-phase spacing $(\delta)$ can be chosen when their effects on CDR performance are analytically understood. A coherent framework for CDR design can thus be established.

- All available phase detectors can be analyzed using a unified methodology. The digital, the oversampling and the linear PDs can be thought of as a continuum determined by their oversampling ratio. An optimum $O S R$ must be chosen for the CDR system in the presence of various types of input jitter to guarantee a certain JTOL and JTRAN performance.

- Exact time-averaged equations have been derived for phase detectors in the presence of random, deterministic and asymmetric jitter while using oversampling ratio $(O S R)$ and inter-phase spacing $(\delta)$ as parameters. This is a clear advantage over prior art. 
- A behavioral simulation flow has been developed that uses the exact phase detector equations derived in this work to facilitate the system-level behavioral simulations for oversampling CDR circuits.

- A novel all-digital Multiple-Rotating-Clock-Phase data recovery architecture has been proposed that establishes a robust interphase spacing by re-using the phases available from the multi-phase oscillator. The same architecture has been implemented on an FPGA.

- Novel techniques have been proposed to shorten the simulation time for otherwise computation-intensive jitter tolerance (JTOL) characterization by exploiting the phase of the jitter sinusoid. The main idea is to exploit the highest slope of the jitter sinusoid that causes transient bit errors.

\subsection{Summary}

This chapter reviewed the main points of this dissertation and presented the contributions from this work. 
To see a world in a grain of sand,

And a heaven in a wild flower,

Hold infinity in the palm of your hand,

And eternity in an hour.

Robert Frost

\subsection{Chapter overview}

This chapter presents the short-term digital CDR research possibilities. Since the field of IC design is constantly evolving, some general observations are listed as a preamble to set the stage for this discussion.

\subsection{Current state of technology}

It is obvious that IC design technology spends a vast fraction of its attention-span on dealing with the uncontrollability, unpredictability and the cost irrationalities of the semiconductor manufacturing industry. Innovation will chart the way into the forseeable future $[136][137][138]$ until a replacement for the silicon substrate is found. The last year of steady gains in CPU raw speeds are history [140][141] and the age of the chip multiprocessor (CMP) is upon us [143]. Unconventional computation mechanisms [15] will have to be tapped for steady gains.

The CMOS technology is the result of the culmination of many supporting sciences such as chemistry, mathematics, optics, and technologies such as processing, photography, lithography, control-systems, business strategies and everything else that has advanced manufacturing and enhanced yields in other areas of human endeavor. Replacing it would require justification on so many more fronts such as human resources, device 
modeling, simulation capacity and the availability of testing equipment. To sustain technological growth, that is taken for granted by the populace, continuous growth is assumed in IC industry. This expectation has reached physical barriers.

In the future, the choice of substrate might be determined by the non-availability of materials as the known supplies of rare-earth materials requried for VLSI fabrication are steadily dwindling and new sources need to be found. Geo-political factors may limit the growth of the VLSI industry in unforseeable ways. Peak Oil will, unceremoniously, disrupt the exponential dreams of many individuals and corporations.

\subsection{Short-term digital CDR research goals}

Following is a list of short-term CDR-related research goals:

- Implementation of a CDR test-vehicle with a custom DCO and DFE along with a programmable digital core as proposed in Figure 3.25(b) - that is being repeated as Figure 7.1 for readability. This has commercial potential for FPGA vendors offering SerDes and wireless solutions.

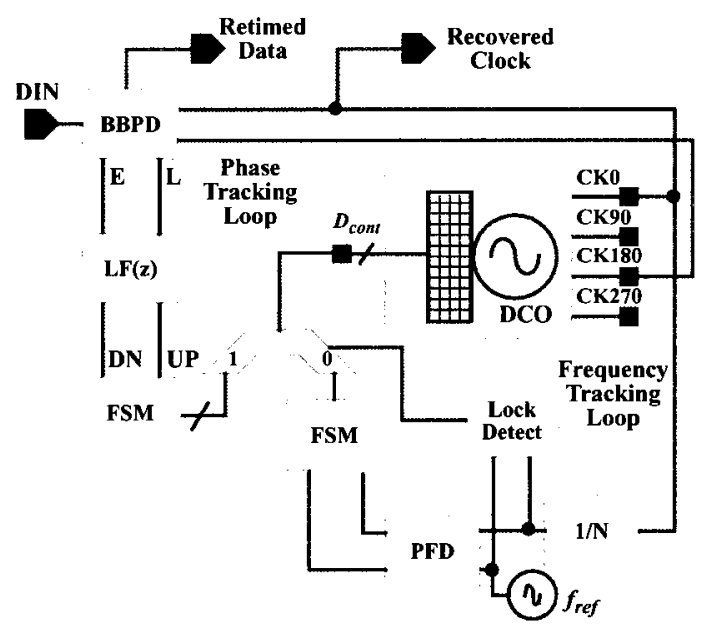

Figure 7.1 Proposed DCO-based CDR architecutre

- There is a rich store of knowledge in the control systems area that talks about detection, estimation and prediction in the presence of noise - white and colored. Unfortunately, there is no one-to-one correspondence between nomenclature 
across electronics and control systems literature. This can lead to duplication of work. A concentrated effort to scour control systems literature can only prove beneficial for the PLL and CDR circuits case, since these are nothing but feedback control systems.

- Further analysis can be done to predict acquisition time and locking range in the presence of different types of jitter for oversampled CDR systems.

- A library can be created containing various jittered waveforms from different channels and DFEs such that a standard simulation regimen can be applied to a new CDR architecture in parallel. This strategy can be beneficial due to the multicore scenario in the near future.

- The use of oversampling PDs in conjunction with various scrambling and coding schemes can be studied such that JTOL higher than 1.0 UI is realized. Synthesizable CDR architectures with high reliability can thus be produced. A lower $O S R$ can perhaps be employed in conjunction with coding.

- Further improvements can be made to the JTOL testbench by encapsulating it inside a scripting language testbench, e.g.; Spectre-MDL to automate the procedure. In this regard, a combined frequency and amplitude chirp can be applied to investigate a further reduction in simulation time.

- An end-to-end simulation of a SerDes system can thus be undertaken using digital simulators. A similar study has been reported by Texas Instruments in conjunction with the DCO based GPS receiver [12]. A similar methodology for the case of digital SerDes can be a research project.

- The metastability of flipflops has been mentioned and further research can prove to be helpful for many areas of IC design including CDR and SerDes chips.

\subsection{Summary}

This chapter presented a list of future research topics that can benefit oversampled, digital CDR and SerDes architectures in the age of multi-core CPUs. 


\section{References}

\section{\#1 Bibliography}

[1] B. Razavi; Design of Analog CMOS Integrated Circuits, McGraw-Hill, 2000

[2] B. Razavi; Monolithic Phase-Locked Loops and Clock Recovery Circuits-Theory and Design, 1996, IEEE Press

[3] D. Johns; K. Martin; Analog Integrated Circuit Design, John Wiley and Sons, 1996

[4] C. Toumazou; Trade-Offs in Analog Circuit Design, The Designer's Companion, Kluwer 2002

[5] S-C. Liu; et al.; Analog VLSI: Circuits and Principles; MIT Press 2002

[6] D. R. Stauffer; et al.; High Speed SerDes Devices and Applications, Springer 2008

[7] R. Steel; Delta Modulation Systems, Pentech Press, London 1975

[8] G. Bourdopoulos; et al.; Delta-Sigma Modulators-Modeling, Design and Applications, Imperial College Press, 2003

[9] M. P. Li; Jitter, Noise and Signal Integrity at High-Speed, Prentice Hall, 2007

[10] S. Lin; Essential Issues in SoC Design; Springer 2006

[11] J. M. Rabaey; A. Chandrakasan; B. Nikolic; Digital Integrated Circuits, 2nd ed. Prentice Hall 2003

[12] R. B. Staszewski; P. T. Balsara; All-Digital Frequency Synthesizer in Deep-Submicron CMOS, John Wiley \& Sons, 2006

[13] C. R. Paul, "Analysis of Multi-conductor Transmission Lines", $2^{\text {nd }}$ edition, John Wiley and Sons, 2008

[14] Z. Vukic, L. Kuljaca; D. Donlagic; S. Tesnjak; Nonlinear Control Systems, Marcel Dekker 2003

[15] A. Adamatzky, C. Teuscher; From Utopian to Genuine Unconventional Computers, Luniver Press 2006

[16] C-C. Yu; Autotuning of PID Controllers-A Relay Feedback Approach, 2nd edition, Springer 2006 


\section{\#2 Publications from this work}

[17] S. I. Ahmed, T. A. Kwasniewski; "An All-Digital Data Recovery Circuit Optimization Using Matlab/Simulink", IEEE ISCAS 2005

[18] S. I. Ahmed, T. A. Kwasniewski; "Overview of Oversampling Clock and Data Recovery Circuits", IEEE CCECE 2005

[19] S. I. Ahmed, Kent Orthner, T. A. Kwasniewski; "Behavioral Test Benches for Digital Clock and Data Recovery Circuits using Verilog-A", IEEE CICC 2005

[20] S. I. Ahmed, T. A. Kwasniewski; "A Multiple-Rotating-Clock-Phases Architecture for Digital Data Recovery Circuits using Verilog-A", IEEE BMAS 2005

[21] S. I. Ahmed, T. A. Kwasniewski; "An efficient jitter tolerance simulation methodology", IEEE MWSCAS 2007

\section{\#3 Other references in the dissertation}

[22] B. Razavi; "Challenges in the Design of High-Speed Clock and Data Recovery Circuits", IEEE Communications Magazine, August 2002

[23] B. Razavi; "Prospecis of CMOS technology for high-speed optical communication circuits", IEEE JSSC 2002

[24] A. C. Sharpe; "A 3-state phase detector can improve your next PLL design", EDN Magazine, September 20, 1976

[25] C. R. Hogge; "A self-correcting clock recovery circuit", IEEE J.Lightwave Technology, December 1985

[26] J. D. H. Alexander; "Clock Recovery from Random Binary Data", Elect. Lett., Vol. 11 , October 1975

[27] M. Hsieh, G. Sobelman, "Architectures for Multi-Gigabit Wire-Linked Clock and Data Recovery”, IEEE CAS Magazine, Fourth Quarter, 2008

[28] G. Idei; et al; "A Clock Recovery PLL Applicable to Data Stream with Missing Pulses", The 2000 IEEE Asia-Pacific Conference on Circuits and Systems, 4-6 December 2000

[29] G. Idei; H. Kunieda; "A False-Lock-Free Clock/Data Recovery PLL for NRZ Data Using Adaptive Phase Frequency Detector”, IEEE Trans. CAS II, Nov 2003

[30] S. Soliman; F. Yuan; K. Raahemifar; "An Overview of Design Techniques for CMOS Phase Detectors", IEEE ISCAS, 2002

[31] K. Murata; T. Otsuji; "A Novel Clock Recovery Circuit for Fully Monolithic Integration", IEEE Transactions on MTT, December 1999

[32] R. C. Walker, "Designing Bang-Bang PLLs for Clock and Data Recovery in Serial Data Transmission Systems" - appears in Phase-Locking in High Performance Systems, IEEE Press (2003) edited by Behzad Razavi

[33] J. Lee; K. S. Kundert; B. Razavi; "Modeling of Jitter in Bang-Bang Clock and Data Recovery Circuits, IEEE CICC, 2003 
[34] J. Lee; K. S. Kundert; B. Razavi; “Analysis and Modeling of Bang-Bang Clock and Data Recovery Circuits”, IEEE JSSC, September 2004

[35] K. Kundert; "Verification of Bit-Error Rate in Bang-Bang Clock and Data Recovery Circuits", Designer's Guide Community, www.designers-guide.org, Version $1 \mathrm{c}, 4$ May 2010

[36] W. Rosenkranz; "Phase-Locked Loops with Limiter Phase Detectors in the Presence of Noise", IEEE Trans. Communications, October 1982

[37] L. Lin; Design Techniques for High Performance Integrated Frequency Synthesizers for Multi-standard Wireless Communication Applications, Ph.D. Dissertation, U. C. Berkeley 2000

[38] K. Muhammad; et al.; "The First Fully Integrated Quad-Band GSM/GPRS Receiver in 90-nm Digital CMOS Process", IEEE JSSC, August 2006

[39] K. Vichienchom; W. Liu; "Analysis of Phase Noise due to Bang-bang Phase Detector in PLL-based Clock and Data Recovery Circuits", IEEE ISCAS, 2003

[40] Toifl, T.; et al.; "A 0.94-ps-RMS-Jitter 0.016- $\mathrm{mm}^{2}$ 2.5-GHz Multiphase Generator PLL with $360^{\circ}$ Digitally Programmable Phase Shift for 10-Gb/s Serial Links," IEEE JSSC, December 2005

[41] M. Chan, A. Postula, "Transient analysis of bang-bang phase locked loops", IET Circuits, Devices Syst. 2009

[42] S-H. Lin, S-I. Liu, "Full-Rate Bang-Bang Phase/Frequency Detectors for Unilateral Continuous-Rate CDRs", IEEE T. Circuits and Systems II, December 2008

[43] J. L. Sonntag, J. Stonick, "A Digital Clock and Data Recovery Architecture for Multi-Gigabit/s Binary Links," CICC 2005

[44] J. L. Sonntag, J. Stonick, "A Digital Clock and Data Recovery Architecture for Multi-Gigabit/s Binary Links”, IEEE JSSC, August 2006

[45] J. Sonntag; R. Leonowich; "A monolithic CMOS $10 \mathrm{MHz}$ DPLL for burst-mode data retiming", ISSCC, 1990

[46] Y. Choi; D-K. Jeong; W. Kim; "Jitter Transfer Analysis of Tracked Oversampling Techniques for Multigigabit Clock and Data Recovery", IEEE Trans. CAS-II, Ana$\log$ and Digital Signal Processing, 2003

[47] H. Abdel-Maguid, Analysis of Bang-Bang Clock and Data Recovery Circuits, Masters Thesis, Carleton University, Ottawa ON, September 2005

[48] Y. Miki et al, "A 50-mW/ch 2.5Gb/s/ch Data Recovery Circuit for the SFI-5 Interface With Digital Eye-Tracking”, IEEE JSSC, April 2004

[49] S-H. Lee; M-S Hwang.; et al; "A 5-Gb/s 0.25- $\mu \mathrm{m}$ CMOS Jitter-Tolerant VariableInterval Oversampling Clock/Data Recovery Circuit", IEEE JSSC, December 2002

[50] S-H. Lee; M-S Hwang.; et al;, "A 5-Gb/s 0.25- $\mu \mathrm{m}$ CMOS Jitter-Tolerant VariableInterval Oversampling Clock/Data Recovery Circuit", IEEE ISSCC 2002

[51] J. Kim; D-K Jeong; "Multi-Gigabit-Rate Clock and Data Recovery Based on Blind Oversampling", IEEE Communications Mag., December 2003 
[52] C-K. K. Yang; “Design of High-Speed Serial Links in CMOS”, Ph.D. Dissertation, CSL-TR-98-775, December 1998, Stanford University, California

[53] M. v. Ierssel; et al.; "A $3.2 \mathrm{~Gb} / \mathrm{s}$ CDR Using Semi-Blind Oversampling to Achieve High Jitter Tolerance", IEEE JSSC, 2007

[54] M. Kubicek; Z. Kolka; "Blind Oversampling Data Recovery with Low Hardware Complexity", Radio Engineering, Vol 19, No. 1, April 2010

[55] M. Mansuri; D. Liu; C. Yang; "Fast frequency acquisition phase-frequency detectors for Gsamples/s phase-locked loops", IEEE JSSC 2002

[56] P. K. Hanumolu; G-Y. Wei; U-K. Moon; "A Wide-Tracking Range Clock and Data Recovery Circuit", IEEE JSSC, 2008

[57] P. K. Hanumolu; M. G. Kim, G-Y. Wei; U-K. Moon; “A 1.6Gbps Digital Clock and Data Recovery Circuit", IEEE CICC 2006

[58] J. C. Scheytt; G. Hanke; U. Langmann; "A 0.155-, 0.622-, and 2.488-Gb/s Automatic Bit-Rate Selecting Clock and Data Recovery IC for Bit-Rate Transparent SDH Systems", IEEE JSSC 1999

[59] M. Horowitz; C-K. K. Yang; S. Sidiropoulos; "High-Speed Electrical Signaling: Overview and Limitations", IEEE Micro, 1998

[60] P. Larsson; "A 2-1600 MHz CMOS Clock Recovery PLL with Low-Vdd Capability", IEEE J. Solid-State Circuits, Vol. 34, No. 12, December 1999, pp. 1951-1960

[61] J. M. Khoury; K. R. Lakshmikumar; "High-Speed Serial Transceiver for Data Communication Systems", IEEE Comms Magazine, 2001

[62] N. D. Dalt; Theory and Implementation of Digital Bang-Bang Frequency Synthesizers for High Speed Serial Data Communications, Ph.D. Dissertation, Electrotechnik und Informationstechnik der Rheinisch-Westfälischen Technischen Hochschule Aachen, Germany 2007

[63] S. Tertinek; J. P. Gleeson; O. Feely; "Statistical Analysis of First-Order Bang-Bang Phase-Locked Loops Using Sign-Dependent Random-Walk Theory”, IEEE Trans. CAS-I, Regular Papers, 2010

[64] A. Vareljian; "Nonlinear behavior study in digital bang-bang PLL", IEEE CICC 2009

[65] N. D. Dalt; "Markov Chain-Based Derivation of the Phase Detector Gain in BangBang PLLs", IEEE Trans. CAS-II, Express Briefs, 2006

[66] B. Chun; M. P. Kennedy; "Statistical Properties of First-Order Bang-Bang PLL With Nonzero Loop Delay", IEEE Trans. CAS-II, Express Briefs, 2008

[67] L. K. Wong; “Analyzing High-Speed Serial Links”, Comms Design, September 22, 2004, articleID $=47900618$

[68] J. L. Shin; et al; "A 40nm 16-Core 128-Thread CMT SPARC SoC Processor", IEEE ISSCC 2010

[69] O' Mahony; et al; “A 47x10Gbps $1.4 \mathrm{~mW} /(\mathrm{Gb} / \mathrm{s})$ Parallel Interface in $45 \mathrm{~nm}$ CMOS", ISSCC 2010

[70] G. Clapp; et al; "Management of switched systems at 100 Tbps: The DARPA CORONET program", Photonics in Switching, Int'l Conf. 2009 
[71] S. Fuller; T. Cox; "Anatomy of a forward-looking open standard [RapidIO]", Computer, Vol. 35, No. 1, January 2002

[72] M. Wang, D. Telian; "New Techniques for Designing and Analyzing Multi-GigaHertz Serial Links", DesignCon 2005

[73] G. Xiang; K. Sheach; P. Brunet; "A Study on High-density High-Speed SerDes Design in Buildup Flip Chip Ball Grid Array Packages", EMPC 2009

[74] A. Ghiasi; " $4 \times 10 \mathrm{G}$ vs Serial $40 \mathrm{G}$ SerDes Maturity and Cost", a presentation given to the IEEE 802.3ba, Interim Meeting Seoul 2008

[75] A. U. Bhobe, P. Sochoux, "A Study of Grounded-Heatspreader for EMI Mitigation of ASIC IC Package", IEEE EMC 2008

[76] R-J. Lee; H-M. Chen; "Efficient Package Pin-Out Planning With System Interconnects Optimization for Package-Board Codesign", IEEE Trans. VLSI Systems, February 2010

[77] M. Z. Straayer; M. H. Perrot; "A Multi-Path Gated Ring Oscillator TDC With First-Order Noise Shaping", IEEE JSSC, April 2009

[78] R. Ho; et al; "Optical Interconnect for High-End Computer Systems", IEEE Design and Test of Computers, March 2010

[79] S. Wang; J-J. Chen; "Thermal-aware lifetime reliability in multicore systems", ISQED, April 2010

[80] B. Eklow; "IP Testing - The Future Differentiator?", Design, Automation and Test in Europe, Conf. Proceedings, 2004

[81] R. Oikawa; "Package Substrate Built-in Three-dimensional Distributed Matching Circuit For High-Speed SerDes Applications", 58th ECTC, 2008

[82] P. Qu; "Measurement and simulation correlation of backplane SerDes channel",

[83] R. Dobkin; et al; "Asynchronous Current Mode Serial Communication", IEEE Trans. VLSI Systems, 2010

[84] R. Dobkin; et al; "Parallel vs. Serial On-Chip Communication", SLIP 2008

[85] C. E. Berndt; T. A. Kwasniewski; "A Review of Common Receive-End Adaptive Equalization Schemes and Algorithms for a High-Speed Serial Backplane", IWSOC 2005

[86] S. U. H. Qureshi; "Adaptive Equalization", Proc. IEEE, 1985, http://ieeexplore.ieee.org/stamp/stamp.jsp?arnumber $=01457566$

[87] W. Wong et al.; "Digitally Assisted Adaptive Equalizer in 90nm With Wide Range Support From 2.5Gbps-6.5Gbps", DesignCon 2007

[88] M. Hsieh; J. Welch; G. Sobelman; "PLL performance comparison with application to spread spectrum clock generator design", Analog Integrated Circuits for Signal Processing, Springer 2009

[89] X. Maillard.; et al; "A 900-Mb/s CMOS Data Recovery DLL Using Half-Frequency Clock", IEEE J. Solid-State Circuits, vol. 37, No. 6, Jun 2002, pp. 711-715

[90] W. Rhee; et al.; "A 10-Gb/s CMOS Clock and Data Recovery Circuit Using a Secondary Delay-Locked Loop", IEEE CICC 2003 
[91] D. Dalton; et al.; "A 12.5-Mb/s to 2.7-Gb/s Continuous-Rate CDR With Automatic Frequency Acquisition and Data-Rate Readback", IEEE JSSC, 2005

[92] S. B. Anand; B. Razavi; "A CMOS Clock Recovery Circuit for 2.5-Gb/s NRZ Data", IEEE J. Solid-State Circuits, Vol. 36, NO. 3, March 2001, pp. 432-439

[93] S. B. Anand; "High Speed Clock and Data Recovery Circuits for Random NonReturn-to-Zero Data", Ph.D. Dissertation, UCLA, 200, UMI Number: 3024052

[94] T. Iwata; et al; "A 5 Gbps CMOS Frequency Tolerant Multi-phase Clock Recovery Circuit", IEEE Symposium in VLSI Circuits, Digest of Technical Papers, June 2002

[95] H-T. Ng; R. Farjad-Rad; et al; “A Second-Order Semi-digital Clock Recovery Circuit Based on Injection Locking", IEEE JSSC, December 2003

[96] Z. Mao; T. H. Szymanski; A 4Gb/s CMOS Fully-Differential Analog Dual DelayLocked Loop Clock/Data Recovery Circuit", IEEE International Conference on Electronics, Circuits and Systems, 2003 (ICECS), December 2003

[97] Y. Moon; D-K. Jeong; et al; “A 0.6-2.5-GBaud CMOS Tracked 3x Oversampling Transceiver With Dead-Zone Phase Detection for Robust Clock/Data Recovery", IEEE JSSC, December 2001

[98] R. Kreienkamp; U. Langmann; C. Zimmerman; T. Aoyama; "A 10-Gb/s CMOS clock and data recovery circuit with an analog phase interpolator", IEEE CICC. 2003

[99] J. Savoj; B. Razavi; "A 10-Gb/s CMOS Clock and Data Recovery Circuit With a Half-Rate Linear Phase Detector", IEEE JSSC, May 2001

[100] J. Savoj; B. Razavi; "A 10-Gb/s CMOS Clock and Data Recovery Circuit With a Half-Rate Binary Phase/Frequency Detector", IEEE JSSC, 2003

[101] S. Byun; et al.; "A 10-Gb/s CMOS CDR and DEMUX IC With a Quarter-Rate Linear Phase Detector", IEEE JSSC, Nov 2006

[102] S-J. Song; J. Lee; S. M. Park; H-J. Yoo; "A 4-Gb/s Clock and Data Recovery Circuit Using Four-Phase 1/8-Rate Clock", IEEE JSSC, July 2003

[103] A. Seedher; G. E. Sobelman, "Fractional Rate Phase Detector for Clock and Data Recovery", IEEE SOC, 2003

[104] R. Zhang.; G. S. La Rue; "Clock and Data Recovery Circuits with Fast Acquisition and Low Jitter", IEEE Workshop on Microelectronic and Electron Devices, 2004,

[105] A. Rezayee; K. Martin; "A 9-16 Gb/s Clock and Data Recovery Circuit with ThreeState Phase Detector and Dual-Path Loop Architecture", European Solid-State Circuits Conference, 2003

[106] L. Lunardi; et al.; "A High Speed Burst Mode Optoelectronic Integrated Circuit Photoreceiver Using InP/InGaAs HBT's”, IEEE J. Lightwave Technology, 1994

[107] S. Topliss; D. Beeler; L. Altwegg; "Synchronization for Passive Optical Networks", IEEE J. Lightwave Technology, 1995

[108] J. Terada; et al.; "A $10.3 \mathrm{~Gb} / \mathrm{s}$ Burst-Mode CDR Using a $\triangle \Sigma$ DAC", IEEE JSSC, 2008

[109] A. Tajalli; P. Muller; M. Atarodi; Y. Leblebici; "Analysis and modeling of jitter and frequency tolerance in gated oscillator based CDRs", IEEE ISCAS, 2006 
[110] S. L. J. Gierkink; "A $2.5 \mathrm{~Gb} / \mathrm{s}$ Run-Length-Tolerant Burst-Mode CDR Based on a 1/8th-Rate Dual Pulse Ring Oscillator”, IEEE JSSC, 2008

[111] H. S. Muthali; T. P. Thomas; I. A. Young; "A CMOS 10-Gb/s SONET Transceiver", IEEE JSSC, 2004

[112] Y. Tao; et al.; “A Signal Integrity-based Link Performance Simulation Platform”, IEEE CICC, 2005

[113] M. H. Fields; et al.; "Transceivers and optical engines for computer and datacenter interconnects", OFC/NFOEC, 2010

[114] S. Assefa; F. Xia; Y. A. Vlasov; "CMOS-integrated low-noise germanium waveguide avalanche photodetector operating at $40 \mathrm{Gbps}$ ", OFC/NFOEC, 2010

[115] T. C. Tang; "Experimental studies of metastability behaviors of sub-micron CMOS ASIC flip flops", IEEE ASIC Conf. \& Exhibit, 1991

[116] D. Li; P. Chuang; M. Sachdev; "Comparative analysis and study of metastability on high-performance flip-flops", ISQED 2010

[117] R. Farjad-Rad; C-K. K. Yang; M. Horowitz; T. Lee; "A 0.3- 4 m CMOS 8-Gb/s 4PAM Serial Link Transceiver", Symposium on VLSI Circuits Digest of Technical Papers, 1999

[118] F. A. Musa; A. C. Carusone; "Clock Recovery in High-Speed Multilevel Serial Links", IEEE ISCAS, 2003

[119] F. A. Musa; A. C. Carusone; "Modeling and Design of Multi-level Bang-Bang CDRs in the Presence of ISI and Noise", IEEE Trans. CAS-I, Regular Papers, 2007

[120] J. L. Zerbe; et al.; "Equalization and Clock Recovery for a 2.5-10-Gb/s 2-PAM/4PAM Backplane Transceiver Cell”, IEEE JSSC, December 2003

[121] Y. Yuminaka; et al.; "Multiple-valued data recovery techniques for band-limited channels in VLSI", ISMVL 2002

[122] Y. Yuminaka; et al.; "Equalization Techniques for Multiple-Valued Data Transmission and Their Application", ISMVL 2007

[123] M. Chang; V. P. Roychowdhury; et al; "RF/Wireless Interconnect for Inter- and Intra-Chip Communications", Proceedings of the IEEE, April 2001

[124] T. Kuroda; N. Miura; et al.; "Perspective of Low-Power and High-speed Wireless Inter-Chip Communications for SiP Integration", ESSCIRC 2006

[125] N. Miura; et al.; "A $1 \mathrm{~Tb} / \mathrm{s} 3 \mathrm{~W}$ inductive-coupling transceiver for interchip clock and data link", ISSCC, Digest of Technical Papers, 2006

[126] N. Miura; et al.; "A $1 \mathrm{~Tb} / \mathrm{s} 3 \mathrm{~W}$ Inductive-Coupling Transceiver for 3D-Stacked Inter-Chip Clock and Data Link", IEEE JSSC, 2007

[127] S. Ibrahim; B. Razavi; "Design requirements of 20-Gb/s serial links using multitone signaling”, IEEE ISSCS, 2009

[128] T. H. Lee; J. F. Bulzacchelli; "A 155-MHz Clock Recovery Delay- and PhaseLocked Loop", IEEE JSSC, December 1992

[129] M. Reinhold; C. Dorschky; et al; “A Fully Integrated 40-Gb/s Clock and Data Recovery IC With 1:4 DEMUX in SiGe Technology", IEEE JSSC, December 2001 
[130] A. Pöttbacker; U. Langmann; "A Si Bipolar Phase and Frequency Detector IC for Clock Extraction up to $8 \mathrm{~Gb} / \mathrm{s}$ ", IEEE JSSC, December 1992

[131] H. Nosaka; K. Ishii; "A Fully Integrated 40-Gbit/s clock and data recovery circuit using InP/InGaAs HBTs", Microwave Symposium Digest 2002, IEEE MTT-S International, June 2002

[132] H. Ransijn; P. O’Connor; "A PLL-Based 2.5-Gb/s GaAs Clock and Data Regenerator IC", IEEE JSSC, October 1991

[133] Y. Greshishchev; P. Schvan; "SiGe Clock and Data Recovery IC with Linear-Type PLL for 10-Gb/s SONET Application", IEEE JSSC, 2000

[134] Y. Greshishchev; et al.; "A Fully Integrated SiGe Receiver IC for 10-Gb/s Data Rate", IEEE JSSC, 2000

[135] B. Razavi; “A 2.5-Gb/s 15-mW Clock Recovery Circuit”, IEEE JSSC, April 1996

[136] J. Walko; "Scaling Is Dead, Long Live Innovation", IEE Review, June 2004

[137] Y. Taur; et al; "CMOS Scaling into the nanometer regime", Proceedings of the IEEE, April 1997

[138] E. J. Nowak; et al; "Turning Silicon on its Edge-Double Gate CMOS/FinFET Technology", IEEE Circuits and Devices Magazine, Jan-Feb 2004

[139] L. B. Kish, "End of Moore's law: thermal (noise) death of integration in micro and nano electronics", Physics Letters A, December 2002

[140] D. Deleganes; J. Douglas; B. Kommandur; M. Patyra; "Designing a 3GHz, 130nm, Intel $^{(\circledR)}$ Pentium $^{(\circledR)}$ Processor", Symposium on VLSI Technology 2002, Digest of Technical Papers, June 2002

[141] J. Clabes; et al; "Design and Implementation of the Power5 $5^{\mathrm{TM}}$ microprocessor", IEEE ISSCC, February, 2004

[142] J. Lin; B. Haroun; et al; "A PVT Tolerant $0.18 \mathrm{MHz}$ to $600 \mathrm{MHz}$ self-calibrated digital PLL in 90nm CMOS process", IEEE ISSCC, 2004

[143] R. Kumar; D. M. Tullsen; N. P. Jouppi; P. Ranganathan; "Heterogeneous Chip Multiprocessors", IEEE Computer, 2005

[144] L. Egiziano; et al.; "Performance Analysis of CNT-based interconnects", IEEE NANO, 2009

[145] H. Li; et al.; "Carbon Nanomaterials: The Ideal Interconnect Technology for NextGeneration ICs", IEEE Design and Test of Computers, 2010

[146] N. Srivastava; et al.; Carbon nanotube interconnects: implications for performance, power dissipation and thermal management", IEDM 2005

[147] O. Tigli; M. E. Zaghloul; "A Novel Circular SAW (Surface Acoustic Wave) Device in CMOS", IEEE Sensors 2007

[148] M. Furuhata; et al.; "Development of Monolithic CMOS-SAW oscillator", IEEE Ultrasonic Symposium, 2005

[149] W. B. Kuhn; Design of Integrated, Low Power, Radio Receivers in BiCMOS Technologies, Ph.D. Dissertation, Virginia Tech Libraries, December 1995

[150] C. Devries; R. Mason; "A 0.18- $\mu \mathrm{m}$ CMOS, High Q-Enhanced Bandpass Filter With Direct Digital Tuning", IEEE CICC, 2002 
[151] L. Angrisani; A. Baccigalupi; L. Ferrigno; "Jitter measurements: from theory to practice", IMTC 2000

[152] M. P. Li et al.; “A New Method for Jitter Decomposition Through Its Distribution Tail Fitting", ITC 1999

[153] M. A. Kossel; M. L. Schmatz; "Jitter Measurements of High-Speed Serial Links", IEEE Design and Test of Computers, 2004

[154] G. Foster; "Dual-Dirac, Scope Histograms and BERTScan Measurements -A Primer", BERTScope article, September 2005, www.bertscope.com

[155] J. Redd, "Calculating Statistical Confidence Levels for Error-Probability Estimates", Lightwave Magazine, April 2000

[156] Dense Wavelength Division Multiplexing (DWDM); IEC whitepaper

[157] Agilent Jitter Poster, from www.agilent.com

[158] http://www.itrs.net

[159] www.intel.com

[160] www.wolfram.com 